Supporting Information for:

\title{
Stereoselective Synthesis of 2-Deoxy-furanosides from 2,3-Anhydro-furanosyl Thioglycosides
}

\author{
Dianjie Hou and Todd L. Lowary*,\#
}

Alberta Ingenuity Centre for Carbohydrate Science and Department of Chemistry, The University of Alberta, Gunning-Lemieux Chemistry Centre,

Edmonton, AB T6G 2G2, Canada.

Email: tlowary@ualberta.ca

Table of Contents

\begin{tabular}{|l|r|}
\hline General Methods & S4 \\
\hline Figure S1 & S5 \\
\hline General Procedures for Glycosylations & S5 \\
\hline Represenative Procedure for Raney Nickel Desulfurization & S5 \\
\hline $\begin{array}{l}\text { Characterization Data for New Compounds and Additional } \\
\text { Experimental Details }\end{array}$ & $\mathrm{S} 6-36$ \\
\hline References for Supporting Information & $\mathrm{S} 37$ \\
\hline${ }^{1} \mathrm{H}$ NMR spectrum of $\mathbf{1 0}$ & $\mathrm{S} 38$ \\
\hline${ }^{13} \mathrm{C}$ NMR spectrum of $\mathbf{1 0}$ & $\mathrm{S} 39$ \\
\hline${ }^{1} \mathrm{H}$ NMR spectrum of $\mathbf{1 9}$ & $\mathrm{S} 40$ \\
\hline${ }^{13} \mathrm{C}$ NMR spectrum (APT) of $\mathbf{1 9}$ & $\mathrm{S} 41$ \\
\hline${ }^{1} \mathrm{H}$ NMR spectrum of $\mathbf{2 0}$ & $\mathrm{S} 42$ \\
\hline${ }^{13} \mathrm{C}$ NMR spectrum (APT) of $\mathbf{2 0}$ & $\mathrm{S} 43$ \\
\hline${ }^{1} \mathrm{H}$ NMR spectrum of $\mathbf{2 1}$ & $\mathrm{S} 44$ \\
\hline${ }^{13} \mathrm{C}$ NMR spectrum (APT) of $\mathbf{2 1}$ & $\mathrm{S} 45$ \\
\hline${ }^{1} \mathrm{H}$ NMR spectrum of $\mathbf{2 2}$ & $\mathrm{S} 46$ \\
\hline
\end{tabular}




\begin{tabular}{|c|c|}
\hline${ }^{13} \mathrm{C}$ NMR spectrum (APT) of 22 & S47 \\
\hline${ }^{1} \mathrm{H}$ NMR spectrum of $\mathbf{2 3}$ & $\mathrm{S} 48$ \\
\hline${ }^{13} \mathrm{C}$ NMR spectrum (APT) of $\mathbf{2 3}$ & S49 \\
\hline${ }^{1} \mathrm{H}$ NMR spectrum of $\mathbf{2 4}$ & $\mathrm{S} 50$ \\
\hline${ }^{13} \mathrm{C}$ NMR spectrum (APT) of 24 & S51 \\
\hline${ }^{1} \mathrm{H}$ NMR spectrum of $\mathbf{2 5}$ & S52 \\
\hline${ }^{13} \mathrm{C}$ NMR spectrum (APT) of $\mathbf{2 5}$ & S53 \\
\hline${ }^{1} \mathrm{H}$ NMR spectrum of $\mathbf{2 6}$ & S54 \\
\hline${ }^{13} \mathrm{C}$ NMR spectrum (APT) of 26 & S55 \\
\hline${ }^{1} \mathrm{H}$ NMR spectrum of 27 & S56 \\
\hline${ }^{13} \mathrm{C}$ NMR spectrum (APT) of 27 & S57 \\
\hline${ }^{1} \mathrm{H}$ NMR spectrum of $\mathbf{2 8}$ & S58 \\
\hline${ }^{13} \mathrm{C}$ NMR spectrum (APT) of $\mathbf{2 8}$ & S59 \\
\hline${ }^{1} \mathrm{H}$ NMR spectrum of $\mathbf{2 9}$ & S60 \\
\hline${ }^{13} \mathrm{C}$ NMR spectrum (APT) of 29 & S61 \\
\hline${ }^{1} \mathrm{H}$ NMR spectrum of $\mathbf{3 0}$ & S62 \\
\hline${ }^{13} \mathrm{C}$ NMR spectrum (APT) of $\mathbf{3 0}$ & S63 \\
\hline${ }^{1} \mathrm{H}$ NMR spectrum of $\mathbf{3 1}$ & S64 \\
\hline${ }^{13} \mathrm{C}$ NMR spectrum (APT) of $\mathbf{3 1}$ & S65 \\
\hline${ }^{1} \mathrm{H}$ NMR spectrum of $\mathbf{3 2}$ & S66 \\
\hline${ }^{13} \mathrm{C}$ NMR spectrum (APT) of 32 & S67 \\
\hline${ }^{1} \mathrm{H}$ NMR spectrum of $\mathbf{3 3}$ & S68 \\
\hline${ }^{13} \mathrm{C}$ NMR spectrum (APT) of $\mathbf{3 3}$ & S69 \\
\hline${ }^{1} \mathrm{H}$ NMR spectrum of $\mathbf{3 4}$ & $\mathrm{S} 70$ \\
\hline${ }^{13} \mathrm{C}$ NMR spectrum (APT) of $\mathbf{3 4}$ & S71 \\
\hline${ }^{1} \mathrm{H}$ NMR spectrum of 35 & $\mathrm{~S} 72$ \\
\hline${ }^{13} \mathrm{C}$ NMR spectrum (APT) of $\mathbf{3 5}$ & S73 \\
\hline${ }^{1} \mathrm{H}$ NMR spectrum of $\mathbf{3 6}$ & S74 \\
\hline${ }^{13} \mathrm{C}$ NMR spectrum (APT) of $\mathbf{3 6}$ & $\mathrm{S} 75$ \\
\hline${ }^{1} \mathrm{H}$ NMR spectrum of 37 & S76 \\
\hline
\end{tabular}




\begin{tabular}{|c|c|}
\hline${ }^{13} \mathrm{C}$ NMR spectrum (APT) of $\mathbf{3 7}$ & S77 \\
\hline${ }^{1} \mathrm{H}$ NMR spectrum of $\mathbf{3 8}$ & S78 \\
\hline${ }^{13} \mathrm{C}$ NMR spectrum (APT) of $\mathbf{3 8}$ & S79 \\
\hline${ }^{1} \mathrm{H}$ NMR spectrum of 39 & $\mathrm{~S} 80$ \\
\hline${ }^{13} \mathrm{C}$ NMR spectrum (APT) of $\mathbf{3 9}$ & S81 \\
\hline${ }^{1} \mathrm{H}$ NMR spectrum of $\mathbf{4 0}$ & S82 \\
\hline${ }^{13} \mathrm{C}$ NMR spectrum (APT) of $\mathbf{4 0}$ & S83 \\
\hline${ }^{1} \mathrm{H}$ NMR spectrum of $\mathbf{4 1}$ & S84 \\
\hline${ }^{13} \mathrm{C}$ NMR spectrum (APT) of $\mathbf{4 1}$ & S85 \\
\hline${ }^{1} \mathrm{H}$ NMR spectrum of $\mathbf{4 2}$ & S86 \\
\hline${ }^{13} \mathrm{C}$ NMR spectrum (APT) of $\mathbf{4 2}$ & S87 \\
\hline${ }^{1} \mathrm{H}$ NMR spectrum of $\mathbf{4 3}$ & S88 \\
\hline${ }^{13} \mathrm{C}$ NMR spectrum (APT) of $\mathbf{4 3}$ & S89 \\
\hline${ }^{1} \mathrm{H}$ NMR spectrum of $\mathbf{4 4}$ & S90 \\
\hline${ }^{13} \mathrm{C}$ NMR spectrum (APT) of 44 & S91 \\
\hline${ }^{1} \mathrm{H}$ NMR spectrum of $\mathbf{4 5}$ & S92 \\
\hline${ }^{13} \mathrm{C}$ NMR spectrum (APT) of 45 & S93 \\
\hline${ }^{1} \mathrm{H}$ NMR spectrum of $\mathbf{4 7}$ & S94 \\
\hline${ }^{13} \mathrm{C}$ NMR spectrum (APT) of $\mathbf{4 7}$ & S95 \\
\hline${ }^{1} \mathrm{H}$ NMR spectrum of $\mathbf{4 8}$ & S96 \\
\hline${ }^{13} \mathrm{C}$ NMR spectrum (APT) of $\mathbf{4 8}$ & S97 \\
\hline${ }^{1} \mathrm{H}$ NMR spectrum of $\mathbf{4 9}$ & S98 \\
\hline${ }^{13} \mathrm{C}$ NMR spectrum (APT) of 49 & S99 \\
\hline${ }^{1} \mathrm{H}$ NMR spectrum of $\mathbf{5 0}$ & S100 \\
\hline${ }^{13} \mathrm{C}$ NMR spectrum (APT) of $\mathbf{5 0}$ & S101 \\
\hline${ }^{1} \mathrm{H}$ NMR spectrum of $\mathbf{5 1}$ & S102 \\
\hline${ }^{13} \mathrm{C}$ NMR spectrum (APT) of $\mathbf{5 1}$ & $\mathrm{S} 103$ \\
\hline
\end{tabular}




\section{General Methods}

Solvents used in reactions were purified by successive passage through columns of alumina and copper under argon atmosphere before use. All reagents used in reactions were purchased from commercial sources and were used without further purification unless noted otherwise. All reactions were carried out under a positive pressure of argon atmosphere and monitored by TLC on silica gel G-25 UV $254(0.25 \mathrm{~mm})$ unless stated otherwise. Spots were detected under UV light and/or by charring with a solution of anisaldehyde in ethanol, acetic acid and $\mathrm{H}_{2} \mathrm{SO}_{4}$. Column chromatography was performed on silica gel $60(40-60 \mu \mathrm{m})$. The ratio between silica gel and crude product ranged from 100:1 to 20:1 (w/w). Iatrobeads refers to a beaded silica gel 6RS-8060, which was manufactured by Iatron Laboratories (Tokyo). $\quad{ }^{1} \mathrm{H}$ NMR and ${ }^{13} \mathrm{C}$ NMR APT spectra ${ }^{1}$ were recorded at 400 or $500 \mathrm{MHz} . \quad{ }^{1} \mathrm{H}$ NMR chemical shifts are referenced to TMS $\left(0.0 \mathrm{ppm}, \mathrm{CDCl}_{3}\right)$ or $\mathrm{CD}_{3} \mathrm{OD}$ (4.78 ppm, $\left.\mathrm{CD}_{3} \mathrm{OD}\right) .{ }^{13} \mathrm{C} \mathrm{NMR}$ chemical shifts are referenced to $\mathrm{CDCl}_{3}\left(77.23 \mathrm{ppm}, \mathrm{CDCl}_{3}\right)$ or $\mathrm{CD}_{3} \mathrm{OD}\left(48.9 \mathrm{ppm}, \mathrm{CD}_{3} \mathrm{OD}\right) . \quad{ }^{1} \mathrm{H} \mathrm{NMR}$ data are reported as though they are first order and the peak assignments were made on the basis of $2 \mathrm{D}-\mathrm{NMR}\left({ }^{1} \mathrm{H}-{ }^{1} \mathrm{H}\right.$ COSY and HMQC) experiment. The monosaccharide residues in the disaccharides and trisaccharides are labeled by no prime, prime and double prime as shown in Figure S1 and these labels are maintained in the assignment of NMR spectra of all compounds. Optical rotations were measured at $21 \pm 2{ }^{\circ} \mathrm{C}$ at the sodium D line $(589 \mathrm{~nm})$ and are in unit of deg・mL $(\mathrm{dm} \cdot \mathrm{g})^{-1}$. ESI-MS spectra were carried out on samples suspended in THF or $\mathrm{CH}_{3} \mathrm{OH}$ and added $\mathrm{NaCl}$. Alcohols 11-18 were either commercially available or were prepared as previously reported. ${ }^{2}$ 


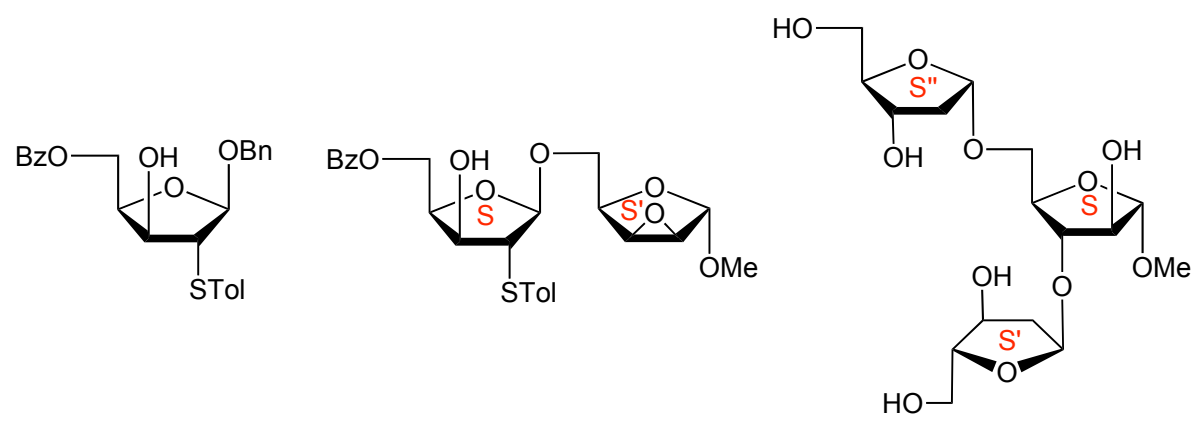

Figure S1.

\section{General Procedures for Glycosylations}

Method A: To a mixture of the alcohol $(0.219 \mathrm{mmol})$, donor $\mathbf{1}^{3}$ or $\mathbf{2}^{3,4}(0.146 \mathrm{mmol})$ and $4 \AA$ molecular sieves $\left(\sim 500 \mathrm{mg}, 10\right.$ equiv. by wt) was added $\mathrm{CH}_{2} \mathrm{Cl}_{2}(10 \mathrm{~mL})$. The mixture was heated at reflux until TLC showed that the donor was consumed. The mixture was concentrated to give a crude residue that was purified by chromatography.

Method B: To a mixture of the alcohol $(0.146 \mathrm{mmol})$, donor $\mathbf{1}^{3}$ or $\mathbf{2}^{3,4}(0.146 \mathrm{mmol})$ and $4 \AA$ molecular sieves $\left(50 \mathrm{mg}, 1\right.$ equiv. by wt) was added $\mathrm{CH}_{2} \mathrm{Cl}_{2}(10 \mathrm{~mL})$. The mixture was stirred for $20 \mathrm{~min}$ at room temperature. Copper triflate $(0.146 \mathrm{mmol})$ was added and the reaction was stirred overnight. After neutralization by triethylamine, the solution was concentrated to yield a crude residue that was purified by chromatography.

\section{Represenative Procedure for Raney Nickel desulfurization ${ }^{5,6}$}

To a solution of compound $19(63 \mathrm{mg}, 0.13 \mathrm{mmol})$ in anhydrous ethanol $(5 \mathrm{~mL})$ was added a catalytic amount of Raney Nickel (W4). The reaction was stirred vigorously under a flow of hydrogen at room temperature until the TLC showed that the starting material was consumed. The catalyst was filtered and the filtrate was concentrated to give a residue that was purified by chromatography in 3:1 hexane-EtOAc to obtain $35(28 \mathrm{mg}, 59 \%)$ as a colorless oil. 
Characterization Data for New Compounds and Additional Experimental Details

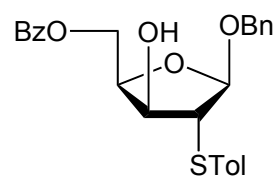

\section{Benzyl 5-O-benzoyl-2-deoxy-2-p-thiotolyl- $\beta$-D-xylofuranoside (10)}

Compound (10) was isolated after chromatography in 6:1 hexane-EtOAc as a colorless oil: $R_{f}$ $0.63\left(2: 1\right.$, hexane-EtOAc); $[\alpha]_{\mathrm{D}}-34.0\left(c\right.$ 1.8, $\left.\mathrm{CH}_{2} \mathrm{Cl}_{2}\right) ;{ }^{1} \mathrm{H}$ NMR $\left(500 \mathrm{MHz}, \mathrm{CDCl}_{3}, \delta_{\mathrm{H}}\right)$ 8.10-8.08 (m, 2H, Ar), 7.59-7.55 (m, 1H, Ar), 7.46-7.42 (m, 2H, Ar), 7.36-7.28 (m, 7H, Ar), 7.15-7.10 (m, 2H, Ar), 5.18 (s, 1H, H-1), 4.82 (d, 1H, $\left.J=11.7 \mathrm{~Hz}, \mathrm{PhCH}_{2}\right), 4.77$ (ddd, 1H, $\left.J_{4,5 \mathrm{~b}}=7.5 \mathrm{~Hz}, J_{4,5 \mathrm{a}}=4.4 \mathrm{~Hz}, J_{4,3}=4.4 \mathrm{~Hz}, \mathrm{H}-4\right), 4.68\left(\mathrm{dd}, 1 \mathrm{H}, J_{5 \mathrm{a}, 5 \mathrm{~b}}=11.8 \mathrm{~Hz}, J_{5 \mathrm{a}, 4}=4.4 \mathrm{~Hz}\right.$, $\mathrm{H}-5 \mathrm{a}), 4.56\left(\mathrm{dd}, 1 \mathrm{H}, J_{5 \mathrm{a}, 5 \mathrm{~b}}=11.8 \mathrm{~Hz}, J_{5 \mathrm{~b}, 4}=7.3 \mathrm{~Hz}, \mathrm{H}-5 \mathrm{~b}\right), 4.52\left(\mathrm{~d}, 1 \mathrm{H}, J=11.7 \mathrm{~Hz}, \mathrm{PhCH}_{2}\right)$, 4.28-4.24 (m, 1H, H-3), $3.79(\mathrm{~s}, 1 \mathrm{H}, \mathrm{H}-2), 3.15\left(\mathrm{~d}, 1 \mathrm{H}, J_{\mathrm{OH}, 3}=11.2 \mathrm{~Hz}, \mathrm{OH}\right), 2.34(\mathrm{~s}, 3 \mathrm{H}$, tolyl $\left.\mathrm{CH}_{3}\right) ;{ }^{13} \mathrm{C}$ NMR $\left(500 \mathrm{MHz}, \mathrm{CDCl}_{3}, \delta_{\mathrm{C}}\right) 166.4(\underline{\mathrm{C}}=\mathrm{O}), 137.9(\mathrm{Ar}), 136.7$ (Ar), 133.1 (Ar), 131.7 $(\mathrm{Ar} \times 2), 130.1(\mathrm{Ar} \times 2), 130.0(\mathrm{Ar}), 129.8(\mathrm{Ar} \times 2), 129.4(\mathrm{Ar}), 128.6(\mathrm{Ar} \times 2), 128.4$ $(\mathrm{Ar} \times 2), 128.1(\mathrm{Ar} \times 2), 128.0(\mathrm{Ar}), 106.2(\mathrm{C}-1), 81.3(\mathrm{C}-4), 75.9(\mathrm{C}-3), 69.9\left(\mathrm{PhCH}_{2}\right), 64.5$ (C-5), 58.2 (C-2), 21.1 (tolyl $\left.\underline{\mathrm{CH}}_{3}\right)$. HRMS (ESI) calcd (M+Na) ${ }^{+} \mathrm{C}_{26} \mathrm{H}_{26} \mathrm{O}_{5} \mathrm{~S}: 473.1393$. Found: 473.1393.

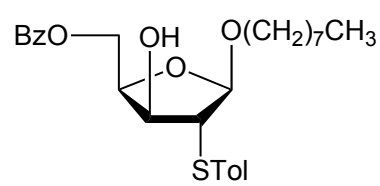

n-Octyl 5-O-benzoyl-2-deoxy-2-p-thiotolyl- $\beta$-D-xylofuranoside (19)

Compound (19) was isolated after chromatography in 5:1 hexane-EtOAc as a colorless oil: $R_{f}$ $0.68\left(2: 1\right.$ hexane-EtOAc); $[\alpha]_{\mathrm{D}}-21.0\left(\right.$ c $\left.1.2, \mathrm{CH}_{2} \mathrm{Cl}_{2}\right) ;{ }^{1} \mathrm{H}$ NMR $\left(500 \mathrm{MHz}, \mathrm{CDCl}_{3}, \delta_{\mathrm{H}}\right)$ 
8.09-8.07 (m, 2H, Ar), 7.58-7.54 (m, 1H, Ar), 7.44-7.41 (m, 2H, Ar), 7.35-7.32 (m, 2H, Ar), 7.15-7.12 (m, 2H, Ar), 5.07 (s, 1H, H-1), 4.74-4.67 (m, 2H, H-4, H-5a), 4.52 (dd, 1H, $J_{5 \mathrm{~b}, 5 \mathrm{a}}=$ $\left.11.5, J_{5 \mathrm{~b}, 4}=8.5 \mathrm{~Hz}, \mathrm{H}-5 \mathrm{~b}\right), 4.25-4.21(\mathrm{~m}, 1 \mathrm{H}, \mathrm{H}-3), 3.77$ (ddd, $1 \mathrm{H}, J=9.6,6.8,6.8 \mathrm{~Hz}$, octyl $\left.\mathrm{OCH}_{2}\right), 3.74(\mathrm{~s}, 1 \mathrm{H}, \mathrm{H}-2), 3.40\left(\mathrm{ddd}, 1 \mathrm{H}, J=9.6,6.8,6.8 \mathrm{~Hz}\right.$, octyl $\left.\mathrm{OCH}_{2}\right), 3.20\left(\mathrm{~d}, 1 \mathrm{H}, J_{\mathrm{OH}, 3}\right.$ $=11.4 \mathrm{~Hz}, \mathrm{OH}), 2.35\left(\mathrm{~s}, 3 \mathrm{H}\right.$, tolyl $\left.\mathrm{CH}_{3}\right), 1.56-1.52\left(\mathrm{~m}, 2 \mathrm{H}\right.$, octyl $\left.\mathrm{CH}_{2}\right), 1.31-1.27(\mathrm{~m}, 10 \mathrm{H}$ octyl), 0.88 (t, 3H, $J=7.1 \mathrm{~Hz}$, octyl $\left.\mathrm{CH}_{3}\right) ;{ }^{13} \mathrm{C} \mathrm{NMR}\left(500 \mathrm{MHz}, \mathrm{CDCl}_{3}, \delta_{\mathrm{C}}\right) 166.4(\underline{\mathrm{C}}=\mathrm{O})$, $137.8(\mathrm{Ar}), 133.0(\mathrm{Ar}), 131.6(\mathrm{Ar} \times 2), 130.1(\mathrm{Ar} \times 2), 130.0(\mathrm{Ar}), 129.8(\mathrm{Ar} \times 2), 129.6(\mathrm{Ar})$, $128.3(\mathrm{Ar} \times 2), 106.9(\mathrm{C}-1), 81.0(\mathrm{C}-4), 75.9(\mathrm{C}-3), 68.4\left(\right.$ octyl $\left.\mathrm{OC}_{2}\right), 64.6(\mathrm{C}-5), 58.0(\mathrm{C}-2)$, 31.8 (octyl $\left.\underline{\mathrm{CH}}_{2}\right), 29.5$ (octyl $\underline{\mathrm{CH}}_{2}$ ), 29.3 (octyl $\left.\underline{\mathrm{CH}}_{2}\right), 29.2$ (octyl $\left.\underline{\mathrm{CH}}_{2}\right), 26.1$ (octyl $\left.\underline{\mathrm{CH}}_{2}\right), 22.6$ (octyl $\underline{\mathrm{CH}}_{2}$ ), 21.1 (tolyl $\underline{\mathrm{CH}}_{3}$ ), 14.1 (octyl $\left.\underline{\mathrm{CH}}_{3}\right)$. HRMS (ESI) calcd $(\mathrm{M}+\mathrm{Na})^{+} \mathrm{C}_{27} \mathrm{H}_{36} \mathrm{O}_{5} \mathrm{~S}$ : 495.2176. Found: 495.2177.

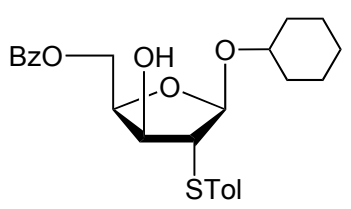

\section{Cyclohexyl 5-O-benzoyl-2-deoxy-2-p-thiotolyl- $\beta$-D-xylofuranoside (20)}

Compound (20) was isolated after chromatography in 6:1 hexane-EtOAc as a colorless oil: $R_{f}$ $0.71\left(2: 1\right.$ hexane-EtOAc); $[\alpha]_{\mathrm{D}}-29.9\left(\right.$ c $\left.2.6, \mathrm{CH}_{2} \mathrm{Cl}_{2}\right) ;{ }^{1} \mathrm{H}$ NMR $\left(500 \mathrm{MHz}, \mathrm{CDCl}_{3}, \delta_{\mathrm{H}}\right)$ 8.09-8.07 (m, 2H, Ar), 7.58-7.54 (m, 1H, Ar), 7.45-7.41 (m, 2H, Ar), 7.36-7.33 (m, 2H, Ar), 7.16-7.13 (m, 2H, Ar), $5.24(\mathrm{~s}, 1 \mathrm{H}, \mathrm{H}-1), 4.74-4.65(\mathrm{~m}, 2 \mathrm{H}, \mathrm{H}-4, \mathrm{H}-5 \mathrm{a}), 4.53\left(\mathrm{dd}, 1 \mathrm{H}, J_{5 \mathrm{~b}, 5 \mathrm{a}}=\right.$ $\left.11.3 \mathrm{~Hz}, J_{5 \mathrm{~b}, 4}=7.1 \mathrm{~Hz}, \mathrm{H}-5 \mathrm{~b}\right), 4.26-4.20(\mathrm{~m}, 1 \mathrm{H}, \mathrm{H}-3), 3.73$ (s, 1H, H-2), 3.70-3.67 (m, 1H, cyclohexyl $\mathrm{OCH}), 3.35\left(\mathrm{~d}, 1 \mathrm{H}, J_{\mathrm{OH}, 3}=11.2 \mathrm{~Hz}, \mathrm{OH}\right), 2.18\left(\mathrm{~s}, 3 \mathrm{H}\right.$, tolyl $\left.\mathrm{CH}_{3}\right), 1.90-1.87(\mathrm{~m}$, $1 \mathrm{H}$, cyclohexyl), 1.84-1.80 (m, 1H, cyclohexyl), 1.71-1.66 (m, 2H, cyclohexyl), 1.52-1.49 
(m, 1H, cyclohexyl), 1.39-1.18 (m, 5H, cyclohexyl); $\left.{ }^{13} \mathrm{C} \mathrm{NMR} \mathrm{(500} \mathrm{MHz,} \mathrm{CDCl}_{3}, \delta_{\mathrm{H}}\right) 166.4$ $(\underline{\mathrm{C}}=\mathrm{O}), 137.6(\mathrm{Ar}), 133.0(\mathrm{Ar}), 131.3(\mathrm{Ar} \times 2), 130.1(\mathrm{Ar} \times 2), 130.0(\mathrm{Ar} \times 2), 129.8(\mathrm{Ar} \times 2)$, $128.3(\mathrm{Ar} \times 2), 104.8(\mathrm{C}-1), 80.8(\mathrm{C}-4), 76.0(\mathrm{C}-3), 75.8(\mathrm{cycclohexyl} \mathrm{O} \underline{\mathrm{H}}), 64.7$ (C-5), 58.0 (C-2), 33.7 (cyclohexyl $\underline{\mathrm{CH}}_{2}$ ), 31.3 (cyclohexyl $\underline{\mathrm{CH}}_{2}$ ), 25.5 (cyclohexyl $\underline{\mathrm{CH}}_{2}$ ), 24.0 (cyclohexyl $\left.\underline{\mathrm{CH}}_{2}\right), 23.7$ (cyclohexyl $\left.\underline{\mathrm{CH}}_{2}\right), 21.1$ (tolyl $\left.\underline{\mathrm{CH}}_{3}\right) . \quad$ HRMS (ESI) calcd $(\mathrm{M}+\mathrm{Na})^{+}$ $\mathrm{C}_{25} \mathrm{H}_{30} \mathrm{O}_{5} \mathrm{~S}: 465.1706$. Found: 465.1709.

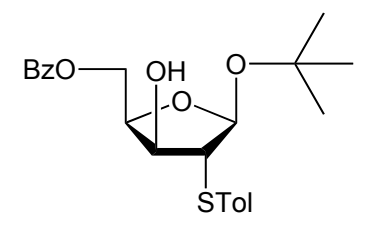

\section{t-Butyl 5-O-benzoyl-2-deoxy-2-p-thiotolyl- $\beta$-D-xylofuranoside (21)}

Compound (21) was isolated after chromatography in (6:1 hexane-EtOAc) as a colorless oil: $R_{f} \quad 0.70\left(2: 1\right.$ hexane-EtOAc); $[\alpha]_{\mathrm{D}}-10.6\left(c, 0.3, \mathrm{CH}_{2} \mathrm{Cl}_{2}\right) ;{ }^{1} \mathrm{H} \mathrm{NMR}\left(500 \mathrm{MHz}, \mathrm{CDCl}_{3}, \delta_{\mathrm{H}}\right)$ 8.10-8.06 (m, 2H, Ar), 7.58-7.54 (m, 1H, Ar), 7.47-7.42 (m, 2H, Ar), 7.35-7.31 (m, 2H, Ar), 7.16-7.12 (m, 2H, Ar), 5.35 (s, 1H, H-1), 4.71-4.65 (m, 2H, H-4, H-5a), 4.59-4.56 (m, 1H, H-5b), 4.25-4.20 (m, 1H, H-3), 3.68 (s, 1H, H-2), 3.38 (d, 1H, $\left.J_{\mathrm{OH}, 3}=11.1 \mathrm{~Hz}, \mathrm{OH}\right), 2.34$ (s, 3H, tolyl $\left.\mathrm{CH}_{3}\right), 1.24\left(\mathrm{~s}, 9 \mathrm{H}, \mathrm{C}\left(\mathrm{CH}_{3}\right)_{3}\right) ;{ }^{13} \mathrm{C} \mathrm{NMR}\left(500 \mathrm{MHz}, \mathrm{CDCl}_{3}, \delta_{\mathrm{C}}\right) 166.4(\underline{\mathrm{C}}=\mathrm{O}), 137.6$ (Ar), $1323.0(\mathrm{Ar}), 131.3(\mathrm{Ar} \times 2), 130.1(\mathrm{Ar} \times 2), 130.0(\mathrm{Ar}), 129.9(\mathrm{Ar} \times 2), 129.8(\mathrm{Ar})$, $128.3(\mathrm{Ar} \times 2), 102.1(\mathrm{C}-1), 80.6(\mathrm{C}-4), 76.5\left(\mathrm{O} \underline{\mathrm{C}}\left(\mathrm{CH}_{3}\right)_{3}\right), 76.1(\mathrm{C}-3), 64.6(\mathrm{C}-5), 58.7(\mathrm{C}-2)$, $28.8\left(\mathrm{C}\left(\underline{\mathrm{CH}}_{3}\right)_{3}\right), 21.1$ (tolyl $\left.\underline{\mathrm{CH}}_{3}\right) . \quad \mathrm{HRMS}(\mathrm{ESI})$ calcd $(\mathrm{M}+\mathrm{Na})^{+} \mathrm{C}_{23} \mathrm{H}_{28} \mathrm{O}_{5} \mathrm{~S}: 439.1550$. Found: 439.1552. 


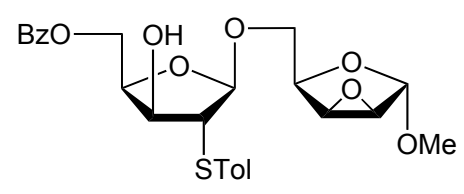

\section{Methyl 5-O-(5-O-benzoyl-2-deoxy-2-p-thiotolyl- $\beta$-D-xylofuranosyl)-2,3-anhydro- $\alpha$-D-}

\section{lyxofuranoside (22)}

|Compound (22) was isolated after chromatography in (4:1 hexane-EtOAc,) as a colorless oil: $R_{f} 0.38\left(2: 1\right.$ hexane-EtOAc); $[\alpha]_{\mathrm{D}}+4.1\left(\right.$ c $\left.5.4 \mathrm{CH}_{2} \mathrm{Cl}_{2}\right) ;{ }^{1} \mathrm{H}$ NMR $\left(500 \mathrm{MHz}, \mathrm{CDCl}_{3}, \delta_{\mathrm{H}}\right)$ 8.10-8.07 (m, 2H, Ar), 7.58-7.54 (m, 1H, Ar), 7.45-7.41 (m, 2H, Ar), 7.36-7.32 (m, 2H, Ar), 7.15-7.11 (m, 2H, Ar), $5.12(\mathrm{~s}, 1 \mathrm{H}, \mathrm{H}-1), 4.98\left(\mathrm{~s}, 1 \mathrm{H}, \mathrm{H}-1^{\prime}\right), 4.75$ (ddd, $1 \mathrm{H}, J_{4,5 \mathrm{a}}=7.9, J_{4,5 \mathrm{~b}}=$ $\left.4.3 \mathrm{~Hz}, J_{4,3}=4.3 \mathrm{~Hz}, \mathrm{H}-4\right), 4.68\left(\mathrm{dd}, 1 \mathrm{H}, J_{5 \mathrm{a}, 5 \mathrm{~b}}=11.8 \mathrm{~Hz}, J_{5 \mathrm{a}, 4}=4.3 \mathrm{~Hz}, \mathrm{H}-5 \mathrm{a}\right), 4.58(\mathrm{dd}, 1 \mathrm{H}$, $\left.J_{5 \mathrm{~b}, 5 \mathrm{a}}=11.8, J_{5 \mathrm{~b}, 4}=7.9 \mathrm{~Hz}, \mathrm{H}-5 \mathrm{~b}\right), 4.26\left(\mathrm{dd}, 1 \mathrm{H}, J_{3, \mathrm{OH}}=11.4 \mathrm{~Hz}, J_{3,4}=4.3 \mathrm{~Hz}, \mathrm{H}-3\right), 4.14(\mathrm{dd}$, $\left.1 \mathrm{H}, J_{4}, 5 \mathrm{a}^{\prime}=5.7 \mathrm{~Hz}, J_{4}{ }^{\prime}, 5 \mathrm{~b}^{\prime}=3.9 \mathrm{~Hz}, \mathrm{H}-4^{\prime}\right), 4.05\left(\mathrm{dd}, 1 \mathrm{H}, J_{5 \mathrm{a}^{\prime}, 5^{\prime} \mathrm{b}}=10.9 \mathrm{~Hz}, J_{5 \mathrm{a}^{\prime}, 4^{\prime}}=3.9 \mathrm{~Hz}\right.$, H-5a'), 3.81 (s, 1H, H-2), 3.72 (dd, 1H, $J_{5 \mathrm{~b}^{\prime}, 5 \mathrm{a}^{\prime}}=10.9 \mathrm{~Hz}, J_{5 \mathrm{~b}}$, $=5.7 \mathrm{~Hz}, \mathrm{H}-5 \mathrm{~b}$ '), 3.68 (d, 1H, $J$ $\left.=2.8 \mathrm{~Hz}, \mathrm{H}-3^{\prime}\right), 3.61\left(\mathrm{~d}, 1 \mathrm{H}, J=2.8 \mathrm{~Hz}, \mathrm{H}-2^{\prime}\right), 3.51$ (d, 1H, $\left.J_{\mathrm{OH}, 3}=11.4 \mathrm{~Hz}, \mathrm{OH}\right), 3.42(\mathrm{~s}, 3 \mathrm{H}$, $\left.\mathrm{OCH}_{3}\right), 2.36\left(\mathrm{~s}, 3 \mathrm{H}\right.$, tolyl $\left.\underline{\mathrm{CH}}_{3}\right) ;{ }^{13} \mathrm{C} \mathrm{NMR}\left(500 \mathrm{MHz}, \mathrm{CDCl}_{3}, \delta_{\mathrm{C}}\right) 166.4(\underline{\mathrm{C}}=\mathrm{O}), 137.8(\mathrm{Ar})$, 133.0 $(\mathrm{Ar} \times 2), 131.8(\mathrm{Ar} \times 2), 130.1(\mathrm{Ar} \times 2), 129.7(\mathrm{Ar} \times 2), 129.5(\mathrm{Ar}), 128.3(\mathrm{Ar} \times 2)$, 106.7 (C-1), 102.2 (C-1'), 81.4 (C-4), 75.9 (C-3), 74.8 (C-4'), 65.9 (C-5'), 64.6 (C-5), 58.1 (C-2), $55.7\left(\mathrm{OCH}_{3}\right), 55.5$ (C-2'), 53.9 (C-3'), $21.1\left(\right.$ tolyl $\left.\mathrm{CH}_{3}\right) . \quad$ HRMS (ESI) calcd (M+Na) ${ }^{+}$ $\mathrm{C}_{25} \mathrm{H}_{28} \mathrm{O}_{8} \mathrm{~S}:$ 511.1397. Found: 511.1394. 


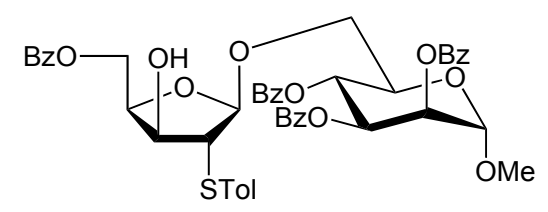

\section{Methyl 5-O-(5-O-benzoyl-2-deoxy-2-p-thiotolyl- $\beta$-D-xylofuranosyl)-2,3,4-tri- $O$-benzoyl-} $\alpha-D-m a n n o p y r a n o s i d e(23)$

Compound (23) was isolated after chromatography in 4:1 hexane-EtOAc as a colorless oil: $R_{f}$ $0.49\left(2: 1\right.$ hexane-EtOAc); $[\alpha]_{\mathrm{D}}-37.2\left(c \quad 0.2, \mathrm{CH}_{2} \mathrm{Cl}_{2}\right) ;{ }^{1} \mathrm{H}$ NMR $\left(500 \mathrm{MHz}, \mathrm{CDCl}_{3}, \delta_{\mathrm{H}}\right)$ 8.12-8.08 (m, 2H, Ar), 8.06-8.02 (m, 2H, Ar), 7.97-7.95 (m, 2H, Ar), 7.84-7.80 (m, 2H, Ar), 7.61-7.33 (m, 12H, Ar), 7.28-7.23 (m, 2H, Ar), 7.16-7.12 (m, 2H, Ar), $5.95\left(\mathrm{dd}, 1 \mathrm{H}, J_{4^{\prime}, 5^{\prime}}=\right.$ $\left.10.0 \mathrm{~Hz}, J_{4^{\prime}, 3^{\prime}}=10.0 \mathrm{~Hz}, \mathrm{H}-4^{\prime}\right), 5.85\left(\mathrm{dd}, 1 \mathrm{H}, J_{3^{\prime}, 4^{\prime}}=10.0 \mathrm{~Hz}, J_{3^{\prime}, 2^{\prime}}=3.3 \mathrm{~Hz}, \mathrm{H}-3^{\prime}\right), 5.70$ (dd, $\left.1 \mathrm{H}, J_{2^{\prime}, 3^{\prime}}=3.3 \mathrm{~Hz}, J_{2}{ }^{\prime}, 1^{\prime}=1.8 \mathrm{~Hz}, \mathrm{H}-2^{\prime}\right), 5.18(\mathrm{~s}, 1 \mathrm{H}, \mathrm{H}-1), 4.97$ (d, $1 \mathrm{H}, J_{1^{\prime}, 2^{\prime}}=1.8 \mathrm{~Hz}, \mathrm{H}-1^{\prime}$ ), $4.77\left(\mathrm{ddd}, 1 \mathrm{H}, J_{4,5 \mathrm{a}}=6.9 \mathrm{~Hz}, J_{4,5 \mathrm{~b}}=4.7 \mathrm{~Hz}, J_{4,3}=4.6 \mathrm{~Hz}, \mathrm{H}-4\right), 4.68-4.61(\mathrm{~m}, 2 \mathrm{H}, \mathrm{H}-5 \mathrm{a}$, H-5b), $4.26\left(\mathrm{dd}, 1 \mathrm{H}, J_{3, \mathrm{OH}}=11.9, J_{3,4}=4.6 \mathrm{~Hz}, \mathrm{H}-3\right), 4.24-4.19(\mathrm{~m}, 1 \mathrm{H}, \mathrm{H}-5$ ') 4.12 (dd, 1H, $\left.J_{6 \mathrm{a}^{\prime}, 6 \mathrm{~b}^{\prime}}=10.9 \mathrm{~Hz}, J_{6 \mathrm{a}^{\prime}, 5}=2.6 \mathrm{~Hz}, \mathrm{H}-6 \mathrm{a}^{\prime}\right), 3.87(\mathrm{~s}, 1 \mathrm{H}, \mathrm{H}-2), 3.62\left(\mathrm{dd}, 1 \mathrm{H}, J_{6 \mathrm{~b}^{\prime}, 6 \mathrm{a}^{\prime}}=10.9 \mathrm{~Hz}\right.$, $\left.J_{6 b^{\prime}, 5}=3.8 \mathrm{~Hz}, \mathrm{H}-6 \mathrm{~b}^{\prime}\right), 3.59\left(\mathrm{~d}, 1 \mathrm{H}, J_{\mathrm{OH}, 3}=11.9 \mathrm{~Hz}, \mathrm{OH}\right), 3.52\left(\mathrm{~s}, 3 \mathrm{H}, \mathrm{OCH}_{3}\right), 2.34(\mathrm{~s}, 3 \mathrm{H}$, tolyl $\left.\mathrm{CH}_{3}\right) ;{ }^{13} \mathrm{C}$ NMR $\left(500 \mathrm{MHz}, \mathrm{CDCl}_{3}, \delta_{\mathrm{C}}\right) 166.3(\underline{\mathrm{C}}=\mathrm{O}), 165.4(\underline{\mathrm{C}}=\mathrm{O} \times 2), 165.4(\underline{\mathrm{C}}=\mathrm{O})$, 137.7 (Ar), 133.4 (Ar), 133.1 (Ar), $133.0(\mathrm{Ar}), 131.5(\mathrm{Ar} \times 2), 130.1(\mathrm{Ar} \times 2), 130.0(\mathrm{Ar} \times 2)$, 129.8 $(\mathrm{Ar} \times 2), 129.74(\mathrm{Ar} \times 4), 129.73(\mathrm{Ar} \times 4), 129.6(\mathrm{Ar}), 129.3(\mathrm{Ar}), 129.2(\mathrm{Ar}), 129.1$ (Ar), $128.5(\mathrm{Ar} \times 2), 128.3(\mathrm{Ar} \times 2), 128.2(\mathrm{Ar} \times 2), 106.4(\mathrm{C}-1), 98.8\left(\mathrm{C}-1^{\prime}\right), 81.6(\mathrm{C}-4)$, 76.0 (C-3), 70.14 (C-2'), 70.09 (C-3’), 69.3 (C-5'), 66.8 (C-4'), 64.6 (C-6’), 64.4 (C-5), 58.2 (C-2), $55.7\left(\mathrm{OCH}_{3}\right), 21.1$ (tolyl, $\left.\underline{\mathrm{CH}}_{3}\right) . \quad \mathrm{HRMS}(\mathrm{ESI})$ calcd $(\mathrm{M}+\mathrm{Na})^{+} \mathrm{C}_{47} \mathrm{H}_{44} \mathrm{O}_{13} \mathrm{~S}:$ 871.2395. Found: 871.2393. 


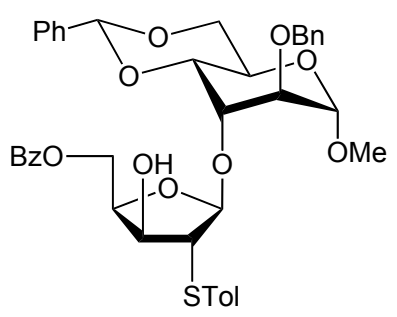

Methyl 3-O-(5-O-benzoyl-2-deoxy-2-p-thiotolyl- $\beta$-D-xylofuranosyl)-2-O-benzyl-4,6-Obenzylidene- $\alpha$-D-altropyranoside (24)

Compound (24) was isolated after chromatography in 4:1 hexane-EtOAc as a colorless oil: $R_{f}$ $0.56\left(2: 1\right.$ hexane-EtOAc); $[\alpha]_{\mathrm{D}}+15.2\left(c\right.$ 3.9, $\left.\mathrm{CH}_{2} \mathrm{Cl}_{2}\right) ;{ }^{1} \mathrm{H}$ NMR $\left(500 \mathrm{MHz}, \mathrm{CDCl}_{3}, \delta_{\mathrm{H}}\right)$ 8.11-8.07 (m, 2H, Ar), 7.59-7.55 (m, 1H, Ar), 7.50-7.37 (m, 6H, Ar), 7.33-7.24 (m, 6H, Ar), 7.22-7.18 (m, 2H, Ar), 6.90-6.86 (m, 2H, Ar), $5.60(\mathrm{~s}, 1 \mathrm{H}, \mathrm{PhCH}), 5.20(\mathrm{~s}, 1 \mathrm{H}, \mathrm{H}-1)$, 4.78-4.70 (m, 3H, H-4, H-5a, H-1'), $4.64\left(\mathrm{~s}, 2 \mathrm{H}, \mathrm{PhCH}_{2}\right), 4.61-4.54(\mathrm{~m}, 1 \mathrm{H}, \mathrm{H}-5 \mathrm{~b})$, 4.33-4.19 (m, 4H, H-3, OH, H-5', H-6a'), 4.10-4.06 (m, 2H, H-2', H-3'), 4.00 (dd, 1H, $J_{4}{ }^{\prime}, 5$ ', $\left.=9.6 \mathrm{~Hz}, J_{4^{\prime}, 3^{\prime}}=2.8 \mathrm{~Hz}, \mathrm{H}-4^{\prime}\right), 3.93(\mathrm{~s}, 1 \mathrm{H}, \mathrm{H}-2), 3.81\left(\mathrm{dd}, 1 \mathrm{H}, J_{6 \mathrm{~b}^{\prime}, 6 \mathrm{a}^{\prime}}=10.2 \mathrm{~Hz}, J_{6 \mathrm{~b}^{\prime}, 5^{\prime}}=10.2\right.$ Hz, H-6b'), 3.42 (s, 3H, $\left.\mathrm{OCH}_{3}\right), 2.24\left(\mathrm{~s}, 3 \mathrm{H}\right.$, tolyl $\left.\mathrm{CH}_{3}\right) ;{ }^{13} \mathrm{C} \mathrm{NMR}\left(500 \mathrm{MHz}, \mathrm{CDCl}_{3}, \delta_{\mathrm{C}}\right)$ $166.3(\underline{\mathrm{C}}=\mathrm{O}), 137.6(\mathrm{Ar}), 137.4(\mathrm{Ar}), 136.8(\mathrm{Ar}), 133.1(\mathrm{Ar}), 130.03(\mathrm{Ar}), 130.0(\mathrm{Ar} \times 2)$, $129.95(\mathrm{Ar}), 129.8(\mathrm{Ar} \times 2), 129.7(\mathrm{Ar} \times 2), 129.1(\mathrm{Ar}), 128.5(\mathrm{Ar} \times 2), 128.4(\mathrm{Ar} \times 2), 128.3$ $(\mathrm{Ar} \times 2), 128.0(\mathrm{Ar}), 127.8(\mathrm{Ar} \times 2), 126.3(\mathrm{Ar} \times 2), 108.7(\mathrm{C}-1), 102.1 \mathrm{PhCH}), 100.1(\mathrm{C}-1$ ' $)$, 81.9 (C-4), 76.9 (C-3’), 75.7 (C-5'), 75.5 (C-4'), 73.8 (C-2'), $73.0\left(\mathrm{PhCH}_{2}\right), 69.3$ (C-6’), 64.3 (C-5), 59.0 (C-3), 57.1 (C-2), $55.4\left(\mathrm{OCH}_{3}\right), 21.0\left(\right.$ tolyl $\left.\underline{\mathrm{CH}}_{3}\right) . \quad$ HRMS (ESI) calcd (M+Na) ${ }^{+}$ $\mathrm{C}_{40} \mathrm{H}_{42} \mathrm{O}_{10} \mathrm{~S}:$ 737.2391. Found: 737.2391. 


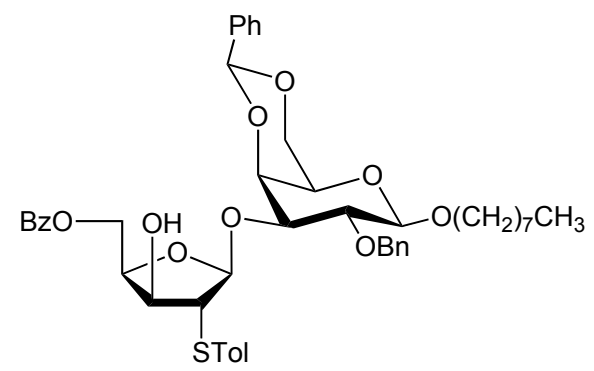

n-Octyl 3-O-(5-O-benzoyl-2-deoxy-2-p-thiotolyl- $\beta$-D-xylofuranosyl)-2-O-benzyl-4,6-Obenzylidene- $\beta$-D-galactopyranoside (25)

Compound (25) was isolated after chromatography in 4:1 hexane-EtOAc as a colorless oil: $R_{f}$ $0.62\left(2: 1\right.$, hexane-EtOAc); $[\alpha]_{\mathrm{D}}+10.0\left(c\right.$ 1.9, $\left.\mathrm{CH}_{2} \mathrm{Cl}_{2}\right) ;{ }^{1} \mathrm{H} \mathrm{NMR}\left(500 \mathrm{MHz}, \mathrm{CDCl}_{3}, \delta_{\mathrm{H}}\right)$ 8.13-8.07 (m, 2H, Ar), 7.62-7.55 (m, 3H, Ar), 7.50-7.44 (m, 2H, Ar), 7.38-7.24 (m, 10H, Ar), 7.07-7.02 (m, 2H, Ar), $5.78(\mathrm{~s}, 1 \mathrm{H}, \mathrm{PhCH}), 5.38(\mathrm{~s}, 1 \mathrm{H}, \mathrm{H}-1), 4.88$ (d, 1H, $J=11.3 \mathrm{~Hz}$ $\left.\mathrm{PhCH}_{2}\right), 4.82-4.75(\mathrm{~m}, 2 \mathrm{H}, \mathrm{H}-4, \mathrm{H}-5 \mathrm{a}), 4.62\left(\mathrm{~d}, 1 \mathrm{H}, J=11.3 \mathrm{~Hz}, \mathrm{PhCH}_{2}\right), 4.52-4.43(\mathrm{~m}, 2 \mathrm{H}$, H-4', H-5b), 4.37 (d, $\left.1 \mathrm{H}, J_{1^{\prime}, 2^{\prime}}=7.7 \mathrm{~Hz}, \mathrm{H}-1^{\prime}\right), 4.33$ (dd, $1 \mathrm{H}, J_{6 \mathrm{a}^{\prime}, 6 \mathrm{~b}^{\prime}}=12.3 \mathrm{~Hz}, J_{6 \mathrm{a}^{\prime}, 5^{\prime}}=1.3 \mathrm{~Hz}$, H-6a'), 4.21-4.15 (m, 1H, H-3), 4.11 (dd, $\left.1 \mathrm{H}, J_{6 \mathrm{~b}^{\prime}, 6 \mathrm{a}^{\prime}}=12.3 \mathrm{~Hz}, J_{6 \mathrm{~b}^{\prime}, 5}{ }^{\prime}=1.6 \mathrm{~Hz}, \mathrm{H}-6 \mathrm{~b}^{\prime}\right), 3.99$ (ddd, 1H, $J=9.4,6.8,6.8 \mathrm{~Hz}$, octyl $\mathrm{OCH}_{2}$ ), 3.76 (s, 1H, H-2), 3.73-3.62 (m, 4H, H-2, H-2', H-3', OH), 3.49 (ddd, 1H, $J=9.4,6.8,6.8 \mathrm{~Hz}$, octyl $\mathrm{OCH}_{2}$ ), 3.43-3.39 (m, 1H, H-5'), 2.24 (s, $3 \mathrm{H}$, tolyl $\left.\mathrm{CH}_{3}\right), 1.72-1.56\left(\mathrm{~m}, 2 \mathrm{H}\right.$, octyl $\left.\mathrm{CH}_{2}\right), 1.45-1.20$ (m, $10 \mathrm{H}$, octyl), 0.89 (t, $3 \mathrm{H}, J=6.7$ $\mathrm{Hz}$, octyl $\left.\mathrm{CH}_{3}\right) ;{ }^{13} \mathrm{C} \mathrm{NMR}\left(500 \mathrm{MHz}, \mathrm{CDCl}_{3}, \delta_{\mathrm{C}}\right) 166.4(\underline{\mathrm{C}}=\mathrm{O}), 138.7(\mathrm{Ar}), 137.8(\mathrm{Ar}), 137.7$ (Ar), 133.2 (Ar), 131.4 ( $\mathrm{Ar} \times 2), 130.1(\mathrm{Ar} \times 2), 130.0(\mathrm{Ar}), 129.7(\mathrm{Ar} \times 2), 129.4(\mathrm{Ar}), 129.0$ $(\mathrm{Ar}), 128.4(\mathrm{Ar} \times 2), 128.3(\mathrm{Ar} \times 2), 128.1(\mathrm{Ar} \times 2), 127.9(\mathrm{Ar} \times 2), 127.6(\mathrm{Ar}), 126.4(\mathrm{Ar} \times$ 2), 108.8 (C-1), 103.5 (C-1'), 101.0 (Phㅡㅐ), 81.7 (C-4), 78.8 (C-2’ or C-3’), 77.8 (C-3’' or C-2'), 75.9 (C-3), 75. 7 (C-4'), 75.1 ( $\left.\mathrm{PhCH}_{2}\right), 70.1$ (octyl $\left.\mathrm{OCH}_{2}\right), 69.0$ (C-6’), 66.3 (C-5'), 64.8 (C-5), 57.8 (C-2), 31.8 (octyl $\underline{\mathrm{CH}}_{2}$ ), 29.7 (octyl $\underline{\mathrm{CH}}_{2}$ ), 29.4 (octyl $\underline{\mathrm{CH}}_{2}$ ), 29.3 (octyl $\underline{\mathrm{CH}}_{2}$ ), 
26.2 (octyl $\left.\underline{\mathrm{CH}}_{2}\right), 22.7$ (octyl $\left.\underline{\mathrm{CH}}_{2}\right), 21.0$ (octyl $\left.\underline{\mathrm{CH}} 2\right), 14.1$ (octyl $\left.\underline{\mathrm{CH}}_{3}\right)$. HRMS (ESI) calcd $(\mathrm{M}+\mathrm{Na})^{+} \mathrm{C}_{47} \mathrm{H}_{56} \mathrm{O}_{10} \mathrm{~S}: 835.3486$. Found: 835.3482.

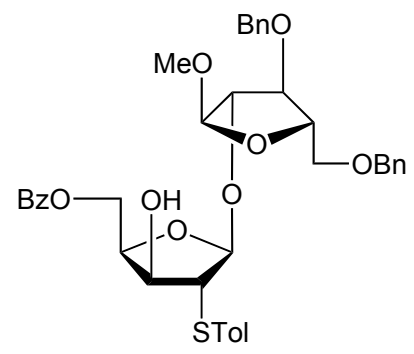

\section{Methyl 2-O-(5-O-benzoyl-2-deoxy-2-p-thiotolyl- $\beta$-D-xylofuranosyl)-3,4-di- $O$-benzyl- $\alpha$ -}

\section{D-arabinofuranoside (26)}

Compound (26) was isolated after chromatography in 4:1 hexane-EtOAc as a colorless oil: $R_{f}$ $0.59\left(2: 1\right.$, hexane-EtOAc); $[\alpha]_{\mathrm{D}}+18.9\left(c \quad 0.3, \mathrm{CH}_{2} \mathrm{Cl}_{2}\right) ;{ }^{1} \mathrm{H}$ NMR $\left(500 \mathrm{MHz}, \mathrm{CDCl}_{3}, \delta_{\mathrm{H}}\right)$ 8.07-8.03 (m, 2H, Ar), 7.58-7.53 (m, 1H, Ar), 7.43-7.38 (m, 2H, Ar), 7.36-7.20 (m, 12H, Ar), 7.18-7.13 (m, 2H, Ar), $5.21(\mathrm{~s}, 1 \mathrm{H}, \mathrm{H}-1), 4.83\left(\mathrm{~s}, 1 \mathrm{H}, \mathrm{H}-1^{\prime}\right), 4.76-4.68$ (m, 3H, H-4, H-5a, $\mathrm{PhCH}_{2}$ ), 4.62-4.46 (m, 5H, $\left.3 \times \mathrm{PhCH}_{2}, \mathrm{H}-5 \mathrm{~b}\right), 4.29-4.27$ (m, 1H, H-2'), 4.24-4.20 (m, 1H, H-3), 4.17-4.11 (m, 1H, H-4'), 4.09-4.04 (m, 1H, H-3'), 3.75 (s, 1H, H-2), 3.60 (dd, 1H, $\left.J_{5 \mathrm{a}^{\prime}, 5 \mathrm{~b}^{\prime}}=10.8 \mathrm{~Hz}, J_{5 \mathrm{a}^{\prime}, 4^{\prime}}=3.0 \mathrm{~Hz}, \mathrm{H}-5 \mathrm{a}^{\prime}\right), 3.51\left(\mathrm{dd}, 1 \mathrm{H}, J_{5 \mathrm{~b}^{\prime}, 5 \mathrm{a}^{\prime}}=10.8 \mathrm{~Hz}, J_{5 \mathrm{~b}^{\prime}, 4^{\prime}}=4.4 \mathrm{~Hz}\right.$,

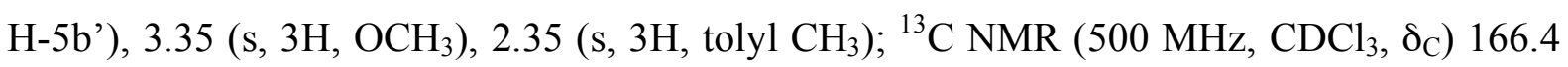
$(\underline{\mathrm{C}}=\mathrm{O}), 138.1(\mathrm{Ar}), 137.8(\mathrm{Ar}), 137.7(\mathrm{Ar}), 133.1(\mathrm{Ar}), 131.9(\mathrm{Ar} \times 2), 130.2(\mathrm{Ar} \times 2), 129.8$ $(\mathrm{Ar}), 129.7(\mathrm{Ar} \times 2), 129.2(\mathrm{Ar}), 128.40(\mathrm{Ar} \times 2), 128.38(\mathrm{Ar} \times 2), 128.3(\mathrm{Ar} \times 2), 127.9(\mathrm{Ar}$ $\times 2), 127.71(\mathrm{Ar} \times 2), 127.69(\mathrm{Ar} \times 2), 107.6(\mathrm{C}-1), 107.4\left(\mathrm{C}-1^{\prime}\right), 86.3\left(\mathrm{C}-2^{\prime}\right), 83.8\left(\mathrm{C}-3^{\prime}\right)$, 81.9 (C-4'), 81.5 (C-4), 75.5 (C-3), $73.4\left(\mathrm{PhCH}_{2}\right), 72.5$ (C-5), 69.2 (C-5'), $64.2\left(\mathrm{PhCH}_{2}\right)$, $58.2(\mathrm{C}-2), 54.8\left(\mathrm{O}_{\mathrm{CH}}^{3}\right), 21.1\left(\right.$ tolyl $\left.\underline{\mathrm{CH}}_{3}\right) . \quad \mathrm{HRMS}(\mathrm{ESI})$ calcd $(\mathrm{M}+\mathrm{Na})^{+} \mathrm{C}_{39} \mathrm{H}_{42} \mathrm{O}_{9} \mathrm{~S}$ : 709.2442. Found: 709.2441. 


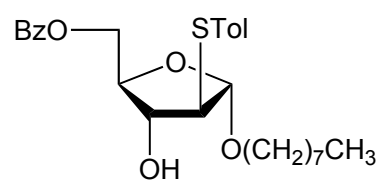

\section{n-Octyl 5-O-benzoyl-2-deoxy-2-p-thiotolyl- $\alpha$-D-arabinofuranoside (27)}

Compound (27) was isolated after chromatography in 5:1 hexane-EtOAc as a colorless oil: $R_{f}$ $0.68\left(2: 1\right.$ hexane-EtOAc); $[\alpha]_{\mathrm{D}}+26.0\left(c\right.$ 4.3, $\left.\mathrm{CH}_{2} \mathrm{Cl}_{2}\right) ;{ }^{1} \mathrm{H}$ NMR $\left(500 \mathrm{MHz}, \mathrm{CDCl}_{3}, \delta_{\mathrm{H}}\right)$ 8.10-8.06 (m, 2H, Ar), 7.59-7.56 (m, 1H, Ar), 7.47-7.42 (m, 2H, Ar), 7.34-7.30 (m, 2H, Ar), 7.13-6.99 (m, 2H, Ar), 5.08 (d, 1H, $\left.J_{1,2}=1.4 \mathrm{~Hz}, \mathrm{H}-1\right), 4.60-4.53$ (m, 2H, H-5a, H-5b), 4.35-4.31 (m, 1H, H-4), $4.07\left(\mathrm{dd}, 1 \mathrm{H}, J_{3,4}=4.7 \mathrm{~Hz}, J_{3,2}=3.4 \mathrm{~Hz}, \mathrm{H}-3\right), 3.74(\mathrm{ddd}, 1 \mathrm{H}, J=$ 9.6, 6.7, $6.7 \mathrm{~Hz}$, octyl $\left.\mathrm{OCH}_{2}\right), 3.60\left(\mathrm{dd}, 1 \mathrm{H}, J_{2,3}=3.4 \mathrm{~Hz}, J_{2,1}=1.4 \mathrm{~Hz}, \mathrm{H}-2\right), 3.42(\mathrm{ddd}, 1 \mathrm{H}$, $J=9.6,6.7,6.7 \mathrm{~Hz}$, octyl $\left.\mathrm{OCH}_{2}\right), 2.40($ br s, $1 \mathrm{H}, \mathrm{OH}), 2.32\left(\mathrm{~s}, 3 \mathrm{H}\right.$, tolyl $\left.\mathrm{CH}_{3}\right), 1.59-1.54(\mathrm{~m}$, 2H, octyl $\left.\mathrm{CH}_{2}\right), 1.32-1.24\left(\mathrm{~m}, 10 \mathrm{H}\right.$, octyl), $0.88\left(\mathrm{t}, 3 \mathrm{H}, J=6.87 \mathrm{~Hz}\right.$, octyl $\left.\mathrm{CH}_{3}\right) ;{ }^{13} \mathrm{C} \mathrm{NMR}$ $\left(500 \mathrm{MHz}, \mathrm{CDCl}_{3}, \delta_{\mathrm{C}}\right) 166.5(\underline{\mathrm{C}}=\mathrm{O}), 137.4(\mathrm{Ar}), 133.1(\mathrm{Ar}), 131.3(\mathrm{Ar} \times 2), 130.1(\mathrm{Ar})$, $130.0(\mathrm{Ar} \times 2), 129.9(\mathrm{Ar} \times 2), 129.8(\mathrm{Ar}), 128.4(\mathrm{Ar} \times 2), 107.3(\mathrm{C}-1), 83.2(\mathrm{C}-4), 77.3(\mathrm{C}-3)$, 68.2 (octyl $\mathrm{OCH}_{2}$ ), 64.5 (C-5), 58.2 (C-2), 31.8 (octyl $\underline{\mathrm{CH}}_{2}$ ), 29.5 (octyl $\underline{\mathrm{CH}}_{2}$ ), 29.3 (octyl $\underline{\mathrm{CH}}_{2}$ ), 29.2 (octyl $\underline{\mathrm{C}} \mathrm{H}_{2}$ ), 26.1 (octyl $\underline{\mathrm{CH}}_{2}$ ), 22.7 (octyl $\underline{\mathrm{CH}}_{2}$ ), 21.1 (tolyl $\underline{\mathrm{CH}}_{3}$ ), 14.1 (octyl $\underline{\mathrm{CH}}_{3}$ ). HRMS (ESI) calcd $(\mathrm{M}+\mathrm{Na})^{+} \mathrm{C}_{27} \mathrm{H}_{36} \mathrm{O}_{5} \mathrm{~S}:$ 495.2176. Found: 495.2177.

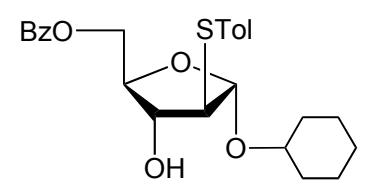

\section{Cyclohexyl 5-O-benzoyl-2-deoxy-2-p-thiotolyl- $\alpha$-D-arabinofuranoside (28)}

Compound (28) was isolated after chromatography in 6:1 hexane-EtOAc as a colorless oil: $R_{f}$ $0.71\left(2: 1\right.$ hexane-EtOAc); $[\alpha]_{\mathrm{D}}+20.7\left(c\right.$ 2.6, $\left.\mathrm{CH}_{2} \mathrm{Cl}_{2}\right) ;{ }^{1} \mathrm{H}$ NMR $\left(500 \mathrm{MHz}, \mathrm{CDCl}_{3}, \delta_{\mathrm{H}}\right)$ 
8.10-8.06 (m, 2H, Ar), 7.59-7.55 (m, 1H, Ar), 7.48-7.42 (m, 2H, Ar), 7.33-7.29 (m, 2H, Ar), 7.13-7.09 (m, 2H, Ar), 5.24 (d, 1H, $J=1.3 \mathrm{~Hz}, \mathrm{H}-1), 4.60-4.53$ (m, 2H, H-5a, H-5b), 4.38-4.34 (m, 1H, H-4), 4.08-4.05 (dd, $\left.1 \mathrm{H}, J_{3,4}=4.2 \mathrm{~Hz}, J_{3,2}=3.2 \mathrm{~Hz}, \mathrm{H}-3\right), 3.66-3.62(\mathrm{~m}$, $1 \mathrm{H}$, cyclohexyl OCH), $3.60\left(\mathrm{dd}, 1 \mathrm{H}, J_{2,3}=3.2 \mathrm{~Hz}, J_{2,1}=1.3 \mathrm{~Hz}, \mathrm{H}-2\right), 2.40$ (br s, $\left.1 \mathrm{H}, \mathrm{OH}\right)$, $2.32\left(\mathrm{~s}, 3 \mathrm{H}\right.$, tolyl $\left.\mathrm{CH}_{3}\right), 1.91-1.87$ (m, 1H, cyclohexyl), 1.84-1.81 (m, 1H, cyclohexyl), 1.74-1.67 (m, 2H, cyclohexyl $\left.\mathrm{CH}_{2}\right), 1.53-1.50(\mathrm{~m}, 1 \mathrm{H}$, cyclohexyl), 1.43-1.20 (m, 5H, cyclohexyl); ${ }^{13} \mathrm{C}$ NMR $\left(500 \mathrm{MHz}, \mathrm{CDCl}_{3}, \delta_{\mathrm{C}}\right) 166.5(\underline{\mathrm{C}}=\mathrm{O}), 137.3$ (Ar), 133.1 (Ar), 131.0 $(\mathrm{Ar} \times 2), 130.2(\mathrm{Ar}), 130.04(\mathrm{Ar} \times 2), 129.97(\mathrm{Ar} \times 2), 129.8(\mathrm{Ar}), 128.3(\mathrm{Ar} \times 2), 105.4$ (C-1), 83.3 (C-4), 77.4 (C-3), 75.6 (cyclohexyl OCيH), 64.6 (C-5), 58.3 (C-2), 33.7 (cyclohexyl $\underline{\mathrm{CH}}_{2}$ ), 31.5 (cyclohexyl $\underline{\mathrm{CH}}_{2}$ ), 25.6 (cyclohexyl $\underline{\mathrm{CH}}_{2}$ ), 24.1 (cyclohexyl $\underline{\mathrm{CH}}_{2}$ ), 23.8 (cyclohexyl $\underline{\mathrm{CH}}_{2}$ ), 21.0 (tolyl $\left.\underline{\mathrm{CH}}_{3}\right)$. HRMS (ESI) calcd $(\mathrm{M}+\mathrm{Na})^{+} \mathrm{C}_{25} \mathrm{H}_{30} \mathrm{O}_{5} \mathrm{~S}$ : 465.1706. Found: 465.1709.

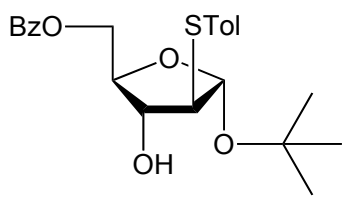

\section{t-Butyl 5-O-benzoyl-2-deoxy-2-p-thiotolyl- $\alpha$-D-arabinofuranoside (29)}

Compound (29) was isolated after chromatography in 6:1 hexane-EtOAc as a colorless oil: $R_{f}$ $0.70\left(2: 1\right.$ hexane-EtOAc); $[\alpha]_{\mathrm{D}}+34.9\left(c \quad 0.1, \mathrm{CH}_{2} \mathrm{Cl}_{2}\right) ;{ }^{1} \mathrm{H}$ NMR $\left(500 \mathrm{MHz}, \mathrm{CDCl}_{3}, \delta_{\mathrm{H}}\right)$ 8.10-8.06 (m, 2H, Ar), 7.59-7.54 (m, 1H, Ar), 7.46-7.42 (m, 2H, Ar), 7.34-7.31 (m, 2H, Ar), 7.13-7.09 (m, 2H, Ar), 5.37 (d, 1H, $\left.J_{1,2}=1.7 \mathrm{~Hz}, \mathrm{H}-1\right), 4.58-4.50$ (m, 2H, H-5a, H-5b), 4.41-4.36 (m, 1H, H-4), 4.07-4.03 (m, 1H, H-3), $3.56\left(\mathrm{dd}, 1 \mathrm{H}, J_{2,3}=3.3 \mathrm{~Hz}, J_{2,1}=1.7 \mathrm{~Hz}\right.$, $\mathrm{H}-2), 2.60$ (br s, $1 \mathrm{H}, \mathrm{OH}), 2.32\left(\mathrm{~s}, 3 \mathrm{H}\right.$, tolyl $\left.\mathrm{CH}_{3}\right), 1.24\left(\mathrm{~s}, 9 \mathrm{H}, \mathrm{C}\left(\mathrm{CH}_{3}\right)_{3}\right) ;{ }^{13} \mathrm{C} \mathrm{NMR}(500$ 
$\left.\mathrm{MHz}, \mathrm{CDCl}_{3}, \delta_{\mathrm{C}}\right) 166.5(\underline{\mathrm{C}}=\mathrm{O}), 137.3(\mathrm{Ar}), 133.1(\mathrm{Ar}), 131.1(\mathrm{Ar} \times 2), 130.3(\mathrm{Ar} \times 2), 130.0$ (Ar), $129.9(\mathrm{Ar} \times 2), 129.8(\mathrm{Ar}), 128.3(\mathrm{Ar} \times 2), 102.5(\mathrm{C}-1), 83.0(\mathrm{C}-4), 77.4(\mathrm{C}-3), 76.0$ $\left(\mathrm{OC}\left(\mathrm{CH}_{3}\right)_{3}\right), 64.8(\mathrm{C}-5), 59.1(\mathrm{C}-2), 28.7\left(\mathrm{C}\left(\underline{\mathrm{CH}}_{3}\right)_{3}\right), 21.0$ (tolyl $\left.\underline{\mathrm{C}} \mathrm{H}_{3}\right) . \quad$ HRMS (ESI) calcd $(\mathrm{M}+\mathrm{Na})^{+} \mathrm{C}_{23} \mathrm{H}_{28} \mathrm{O}_{5} \mathrm{~S}: 439.1550$. Found: 439.1548.

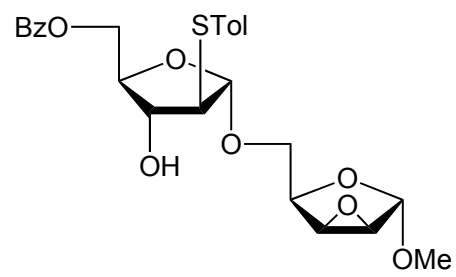

\section{Methyl 5-O-(5-O-benzoyl-2-deoxy-2-p-thiotolyl- $\alpha$-D-xylofuranosyl)-2,3-anhydro- $\alpha$-D-} arabinofuranoside (30)

Compound (30) was isolated after chromatography in 4:1 hexane-EtOAc as a colorless oil: $R_{f}$ $0.38\left(2: 1\right.$, hexane-EtOAc); $[\alpha]_{\mathrm{D}}+4.1$ (c 5.4, $\left.\mathrm{CH}_{2} \mathrm{Cl}_{2}\right) ;{ }^{1} \mathrm{H} \mathrm{NMR}\left(400 \mathrm{MHz}, \mathrm{CDCl}_{3}, \delta_{\mathrm{H}}\right)$ 8.10-8.07 (m, 2H, Ar), 7.60-7.55 (m, 1H, Ar), 7.47-7.42 (m, 2H, Ar), 7.34-7.30 (m, 2H, Ar), 7.13-7.09 (m, 2H, Ar), 5.15 (d, 1H, $J=1.1 \mathrm{~Hz}, \mathrm{H}-1), 4.95$ (s, 1H, H-1'), 4.60-4.54 (m, 2H, H-5a, H-5b), 4.39-4.35 (m, 1H, H-4), 4.18-4.14 (m, 1H, H-4'), 4.12-4.06 (m, 1H, H-3), 3.97 $\left(\mathrm{dd}, 1 \mathrm{H}, J_{5 \mathrm{a}^{\prime}, 5 \mathrm{~b}^{\prime}}=10.3 \mathrm{~Hz}, J_{5 \mathrm{a}^{\prime}, 4^{\prime}}=5.4 \mathrm{~Hz}, \mathrm{H}-5 \mathrm{a}^{\prime}\right), 3.72-3.64$ (m, 4H, H-2, H-2', H-3', H-5b'), $3.40\left(\mathrm{~s}, 3 \mathrm{H}, \mathrm{OCH}_{3}\right), 3.10(\mathrm{br}, 1 \mathrm{H}, \mathrm{OH}), 2.36\left(\mathrm{~s}, 3 \mathrm{H}\right.$, tolyl $\left.\mathrm{CH}_{3}\right) ;{ }^{13} \mathrm{C} \mathrm{NMR}\left(500 \mathrm{MHz}, \mathrm{CDCl}_{3}\right.$, $\left.\delta_{\mathrm{C}}\right) 166.5(\underline{\mathrm{C}}=\mathrm{O}), 137.6(\mathrm{Ar}), 133.1(\mathrm{Ar} \times 2), 131.5(\mathrm{Ar} \times 2), 130.0(\mathrm{Ar} \times 2), 129.9(\mathrm{Ar})$, 129.7 $(\mathrm{Ar} \times 2), 128.4(\mathrm{Ar} \times 2), 107.1(\mathrm{C}-1), 102.1\left(\mathrm{C}-1^{\prime}\right), 83.7(\mathrm{C}-4), 77.2(\mathrm{C}-3), 74.5\left(\mathrm{C}-4^{\prime}\right)$, $65.3\left(\mathrm{C}-5\right.$ ') $64.4(\mathrm{C}-5), 58.2(\mathrm{C}-2), 56.0\left(\mathrm{C}-2^{\prime}\right.$ or $\mathrm{C}-3$ ') $55.5\left(\mathrm{OCH}_{3}\right), 53.9\left(\mathrm{C}-3\right.$ ' or $\left.\mathrm{C}-2^{\prime}\right)$, 21.0 (tolyl $\left.\underline{\mathrm{CH}}_{3}\right) . \quad$ HRMS (ESI) calcd $(\mathrm{M}+\mathrm{Na})^{+} \mathrm{C}_{25} \mathrm{H}_{28} \mathrm{O}_{8} \mathrm{~S}: 511.1397 . \quad$ Found: 511.1398. 


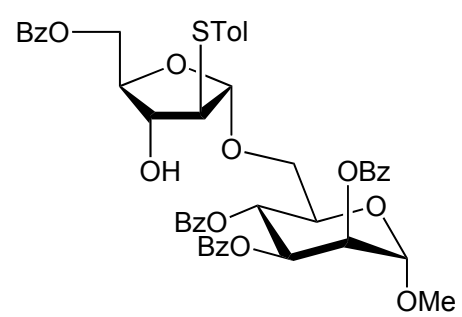

Methyl 5-O-(5-O-benzoyl-2-deoxy-2-p-thiotolyl- $\alpha$-D-arabinofuranosyl)-2,3,4-tri- $O$ benzoyl- $\alpha$-D-mannopyranoside (31)

Compound (31) was isolated after chromatography in 4:1 hexane-EtOAc as a colorless oil: $R_{f}$ $0.49\left(2: 1\right.$ hexane-EtOAc); $[\alpha]_{\mathrm{D}}-17.9\left(c \quad 0.2, \mathrm{CH}_{2} \mathrm{Cl}_{2}\right) ;{ }^{1} \mathrm{H}$ NMR $\left(500 \mathrm{MHz}, \mathrm{CDCl}_{3}, \delta_{\mathrm{H}}\right)$ 8.16-8.11 (m, 2H, Ar), 8.04-8.01 (m, 2H, Ar), 8.00-7.96 (m, 2H, Ar), 7.83-7.79 (m, 2H, Ar), 7.61-7.33 (m, 10H, Ar), 7.29-7.24 (m, 4H, Ar), 7.10-7.08 (m, 2H, Ar), $6.12\left(\mathrm{dd}, 1 \mathrm{H}, J_{4^{\prime}, 5^{\prime}}=\right.$ $\left.10.2 \mathrm{~Hz}, J_{4^{\prime}, 3^{\prime}}=10.2 \mathrm{~Hz}, \mathrm{H}-4^{\prime}\right), 5.89\left(\mathrm{dd}, 1 \mathrm{H}, J_{3^{\prime}, 4^{\prime}}=10.2 \mathrm{~Hz}, J_{3^{\prime}, 2^{\prime}}=3.3 \mathrm{~Hz}, \mathrm{H}-3^{\prime}\right), 5.67$ (dd, $\left.1 \mathrm{H}, J_{2^{\prime}, 3^{\prime}}=3.3 \mathrm{~Hz}, J_{3^{\prime}, 1^{\prime}}=1.9 \mathrm{~Hz}, \mathrm{H}-2^{\prime}\right), 5.21$ (br s, $\left.1 \mathrm{H}, \mathrm{H}-1\right), 4.99$ (d, $1 \mathrm{H}, J_{1^{\prime}, 2^{\prime}}=1.9 \mathrm{~Hz}$, H-1'), 4.55-4.45 (m, 2H, H-5a, H-5b), 4.40-4.35 (m, 1H, H-4), 4.23-4.15 (m, 2H, H-5', H-3), $4.00\left(\mathrm{dd}, 1 \mathrm{H}, J_{6 \mathrm{a}^{\prime}, 6 \mathrm{~b}^{\prime}}=11.1 \mathrm{~Hz}, J_{6 \mathrm{a}^{\prime}, 5^{\prime}},=3.4 \mathrm{~Hz}, \mathrm{H}-6 \mathrm{a}^{\prime}\right), 3.80\left(\mathrm{~d}, 1 \mathrm{H}, J_{2,1}=1.3 \mathrm{~Hz}, \mathrm{H}-2\right), 3.64$ $\left(\mathrm{dd}, 1 \mathrm{H}, J_{6 \mathrm{~b}^{\prime}, 6 \mathrm{a}}{ }^{\prime}=11.1 \mathrm{~Hz}, J_{6 \mathrm{~b}^{\prime}, 5^{\prime}}=2.1 \mathrm{~Hz}, \mathrm{H}-6 \mathrm{~b}^{\prime}\right), 3.52\left(\mathrm{~s}, 3 \mathrm{H}, \mathrm{OCH}_{3}\right), 2.32\left(\mathrm{~s}, 3 \mathrm{H}\right.$, tolyl $\left.\mathrm{CH}_{3}\right)$;

${ }^{13} \mathrm{C} \operatorname{NMR}\left(500 \mathrm{MHz}, \mathrm{CDCl}_{3}, \delta_{\mathrm{C}}\right) 166.3(\underline{\mathrm{C}}=\mathrm{O}), 165.9(\underline{\mathrm{C}}=\mathrm{O}), 165.4(\underline{\mathrm{C}}=\mathrm{O} \times 2), 137.3(\mathrm{Ar})$, $133.6(\mathrm{Ar}), 133.5(\mathrm{Ar}), 133.1(\mathrm{Ar}), 133.0(\mathrm{Ar}), 131.0(\mathrm{Ar} \times 2), 130.1(\mathrm{Ar}), 130.0(\mathrm{Ar} \times 2)$ $129.9(\mathrm{Ar} \times 2), 129.84(\mathrm{Ar} \times 3), 129.78(\mathrm{Ar}), 129.7(\mathrm{Ar} \times 2), 129.3(\mathrm{Ar}), 129.1(\mathrm{Ar}), 128.8$ (Ar), $128.7(\mathrm{Ar} \times 2), 128.5(\mathrm{Ar} \times 2), 128.34(\mathrm{Ar} \times 2), 128.28(\mathrm{Ar} \times 3), 107.6(\mathrm{C}-1), 98.7$ (C-1'), 84.8 C-4), 78.1 (C-3), 70.4 (C-2'), 69.9 (C-3'), 69.4 (C-5’), 66.7 (C-4'), 65.6 (C-6’), $64.9(\mathrm{C}-5), 58.5(\mathrm{C}-2), 55.7\left(\mathrm{OCH}_{3}\right), 21.1\left(\right.$ tolyl $\left.\underline{\mathrm{CH}}_{3}\right) . \quad$ HRMS (ESI) calcd $(\mathrm{M}+\mathrm{Na})^{+}$ $\mathrm{C}_{47} \mathrm{H}_{44} \mathrm{O}_{13} \mathrm{~S}:$ 871.2395. Found: 871.2394. 


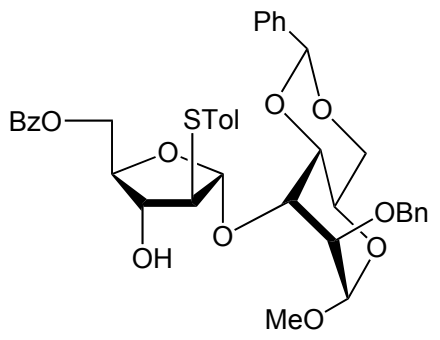

Methyl 3-O-(5-O-benzoyl-2-deoxy-2-p-thiotolyl- $\alpha$-D-arabinofuranosyl)-2- $O$-benzyl4,6-O-benzylidene- $\alpha$-D-altropyranoside (32)

Compound (32) was isolated after chromatography in 4:1 hexane-EtOAc as a colorless oil: $R_{f}$ $0.56\left(2: 1\right.$ hexane-EtOAc); $[\alpha]_{\mathrm{D}}+31.0\left(c \quad 0.1, \mathrm{CH}_{2} \mathrm{Cl}_{2}\right) ;{ }^{1} \mathrm{H}$ NMR $\left(500 \mathrm{MHz}, \mathrm{CDCl}_{3}, \delta_{\mathrm{H}}\right)$ 8.07-8.03 (m, 2H, Ar), 7.59-7.55 (m, 1H, Ar), 7.47-7.41 (m, 5H, Ar), 7.38-7.25 (m, 9H, Ar), 7.12-7.08 (m, 2H, Ar), 5.62 (s, 1H, PhCH), 5.28 (br s, 1H, H-1), 4.70 (s, 1H, H-1'), 4.64 (d, $\left.2 \mathrm{H}, J=11.8 \mathrm{~Hz}, \mathrm{PhCH}_{2}\right), 4.61\left(\mathrm{~d}, J=11.8 \mathrm{~Hz}, \mathrm{PhCH}_{2}\right), 4.50\left(\mathrm{dd}, 1 \mathrm{H}, J_{5 \mathrm{a}, 5 \mathrm{~b}}=11.5 \mathrm{~Hz}, J_{5 \mathrm{a}, 4}=\right.$ $6.4 \mathrm{~Hz}, \mathrm{H}-5 \mathrm{a}), 4.42\left(\mathrm{dd}, 1 \mathrm{H}, J_{5 \mathrm{~b}, 5 \mathrm{a}}=11.5 \mathrm{~Hz}, J_{5 \mathrm{~b}, 4}=5.2 \mathrm{~Hz}, \mathrm{H}-5 \mathrm{~b}\right), 4.39-4.35$ (m, 1H, H-3'), 4.34-4.28 (m, 2H, H-4, H-6a'), 4.25-4.19 (m, 1H, H-5'), 4.07-4.02 (m, 2H, H-3, H-4'), 3.78 $\left(\mathrm{dd}, 1 \mathrm{H}, J_{6 \mathrm{~b}^{\prime}, 6 \mathrm{a}}{ }^{\prime}=10.3, \mathrm{~Hz}, J_{6 \mathrm{~b}^{\prime}, 5}=10.3 \mathrm{~Hz}, \mathrm{H}-6 \mathrm{~b}^{\prime}\right), 3.74\left(\mathrm{~d}, 1 \mathrm{H}, J_{2,1}=0.9 \mathrm{~Hz}, \mathrm{H}-2\right)$, 3.68-3.66 (m, 1H, H-2'), 3.40 (d, $J=11.9 \mathrm{~Hz}, \mathrm{OH}), 3.38\left(\mathrm{~s}, 3 \mathrm{H}, \mathrm{OCH}_{3}\right), 2.32$ (s, 3H, tolyl $\left.\mathrm{CH}_{3}\right) ;{ }^{13} \mathrm{C}$ NMR $\left(500 \mathrm{MHz}, \mathrm{CDCl}_{3}, \delta_{\mathrm{C}}\right) 166.2(\mathrm{C}=\mathrm{O}), 137.11(\mathrm{Ar}), 137.10(\mathrm{Ar}), 136.8$ (Ar), $133.0(\mathrm{Ar}), 130.6(\mathrm{Ar} \times 2), 130.0(\mathrm{Ar} \times 2), 129.89(\mathrm{Ar} \times 2), 129.88(\mathrm{Ar} \times 2), 129.1(\mathrm{Ar})$, 128.6 $(\mathrm{Ar} \times 2), 128.3(\mathrm{Ar} \times 2), 128.2(\mathrm{Ar} \times 2), 128.1(\mathrm{Ar}), 127.8(\mathrm{Ar} \times 2), 126.2(\mathrm{Ar} \times 2)$, 104.0 (C-1), 102.5 (PhCH), 99.8 (C-1'), 85.1 (C-4), 77.8 (C-3 or C-4'), 75.2 (C-4' or C-3), 74.0 (C-2'), 72.8 ( $\left.\mathrm{Ph}^{\mathrm{C}} \mathrm{H}_{2}\right), 69.4$ (C-6'), 67.9 (C-3’), 64.8 (C-5), 58.6 (C-5’), 57.7 (C-2), 55.4 $\left(\mathrm{OCH}_{3}\right), 21.0$ (tolyl $\left.\underline{\mathrm{CH}}_{3}\right)$. HRMS (ESI) calcd $(\mathrm{M}+\mathrm{Na})^{+} \mathrm{C}_{40} \mathrm{H}_{42} \mathrm{O}_{10} \mathrm{~S}: 737.2391$. Found: 737.2398 . 


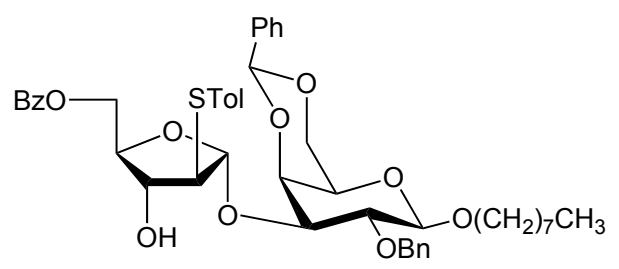

n-Octyl 3-O-(5-O-benzoyl-2-deoxy-2-p-thiotolyl- $\alpha$-D-arabinofuranosyl)-2-O-benzyl-4,6-

\section{$O$-benzylidene- $\beta$-D-galactopyranoside (33)}

Compound (33) was isolated after chromatography in 4:1 hexane-EtOAc as a colorless oil: $R_{f}$

$0.61\left(2: 1\right.$ hexane-EtOAc); $[\alpha]_{\mathrm{D}}+10.0\left(c\right.$ 1.9, $\left.\mathrm{CH}_{2} \mathrm{Cl}_{2}\right) ;{ }^{1} \mathrm{H}$ NMR $\left(500 \mathrm{MHz}, \mathrm{CDCl}_{3}, \delta_{\mathrm{H}}\right)$ 8.10-8.06 (m, 2H, Ar), 7.60-7.55 (m, 1H, Ar), 7.52-7.42 (m, 4H, Ar), 7.40-7.34 (m, 5H, Ar), 7.33-7.18 (m, 5H, Ar), 6.92-6.88 (m, 2H, Ar), 5.47 (s, 1H, PhCH), 5.36 (s, 1H, H-1), 4.99 (d, $\left.1 \mathrm{H}, J=10.8 \mathrm{~Hz}, \mathrm{PhCH}_{2}\right), 4.65\left(\mathrm{~d}, 1 \mathrm{H}, J=10.8 \mathrm{~Hz}, \mathrm{PhCH}_{2}\right), 4.52-4.45$ (m, 3H, H-5a, H-5b, H-1'), 4.35-4.25 (m, 2H, H-4, H-6a', H-4'), 4.11-4.06 (m, 1H, H-3), 4.05 (dd, 1H, J6b',6a' = 12.4 Hz, $\left.J_{6 \mathrm{~b}}, 5^{\prime}=1.7 \mathrm{~Hz}, \mathrm{H}-6 \mathrm{~b} '\right), 4.00$ (ddd, $1 \mathrm{H}, J=9.4,6.8,6.8 \mathrm{~Hz}$, octyl $\mathrm{OCH}_{2}$ ), 3.95 (dd, $\left.1 \mathrm{H}, J_{3^{\prime}, 2^{\prime}}=10.0 \mathrm{~Hz}, J_{3^{\prime}, 4^{\prime}}=3.7 \mathrm{~Hz}, \mathrm{H}-3^{\prime}\right), 3.78\left(\mathrm{dd}, 1 \mathrm{H}, J_{2^{\prime}, 3^{\prime}}=10.0 \mathrm{~Hz}, J_{2^{\prime}, 1^{\prime}}=7.7 \mathrm{~Hz}, \mathrm{H}-2^{\prime}\right)$, $3.71\left(\mathrm{~d}, 1 \mathrm{H}, J_{2,3}=2.0 \mathrm{~Hz}, \mathrm{H}-2\right), 3.51\left(\mathrm{ddd}, 1 \mathrm{H}, J=9.4,6.8,6.8 \mathrm{~Hz}\right.$, octyl $\left.\mathrm{OCH}_{2}\right), 3.42-3.36$ (m, 1H, H-5'), 2.90 (br s, 1H, OH), 2.21 (s, 3H, tolyl $\left.\mathrm{CH}_{3}\right), 1.69-1.55$ (m, 4H, octyl), $1.40-1.25\left(\mathrm{~m}, 11 \mathrm{H}\right.$, octyl), $0.86\left(\mathrm{t}, 3 \mathrm{H}, J=6.9 \mathrm{~Hz}\right.$, octyl $\left.\mathrm{CH}_{3}\right) ;{ }^{13} \mathrm{C} \mathrm{NMR}\left(500 \mathrm{MHz}, \mathrm{CDCl}_{3}\right.$, $\left.\delta_{\mathrm{C}}\right) 166.4(\underline{\mathrm{C}}=\mathrm{O}), 138.3(\mathrm{Ar}), 137.7(\mathrm{Ar}), 136.9(\mathrm{Ar}), 133.1(\mathrm{Ar}), 130.5(\mathrm{Ar}), 130.2(\mathrm{Ar} \times 2)$, 130.0 $(\mathrm{Ar} \times 2), 129.9(\mathrm{Ar} \times 2), 129.8(\mathrm{Ar}), 129.0(\mathrm{Ar}), 128.3(\mathrm{Ar} \times 4), 128.2(\mathrm{Ar} \times 2), 128.1$ $(\mathrm{Ar} \times 2), 127.7(\mathrm{Ar}), 126.4(\mathrm{Ar} \times 2), 104.0(\mathrm{C}-1$ ') $, 103.2(\mathrm{C}-1), 101.0(\mathrm{PhC} H), 84.4(\mathrm{C}-4)$, 77.6 (C-3), 76.6 (C-2'), 74.9 ( $\left.\mathrm{Ph}^{\mathrm{C}} \mathrm{H}_{2}\right), 73.7$ (C-3'), 71.7 (C-4'), 70.1 (octyl $\left.\mathrm{OCH}_{2}\right), 69.3$ (C-6'), 66.2 (C-5'), 64.2 (C-5), 58.0 (C-2), 31.8 (octyl $\underline{\mathrm{CH}}_{2}$ ), 29.7 (octyl $\underline{\mathrm{CH}}_{2}$ ), 29.4 (octyl 
$\underline{\mathrm{CH}}_{2}$ ), 29.2 (octyl $\left.\underline{\mathrm{CH}}_{2}\right), 26.2$ (octyl $\left.\underline{\mathrm{CH}}_{2}\right), 22.7$ (octyl $\left.\underline{\mathrm{CH}}_{2}\right), 21.0$ (tolyl $\left.\underline{\mathrm{CH}}_{3}\right), 14.1$ (octyl $\left.\underline{\mathrm{CH}}_{3}\right)$. HRMS (ESI) calcd (M+Na) $\mathrm{C}_{47} \mathrm{H}_{56} \mathrm{O}_{10} \mathrm{~S}:$ 835.3486. Found: 835.3486.

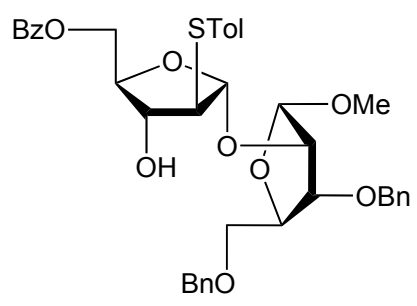

Methyl 2-O-(5-O-benzoyl-2-deoxy-2-p-thiotolyl- $\alpha$-D-arabinofuranosyl)-3,5-di- $O$-benzyl$\alpha$-D-arabinofuranoside (34)

Compound (34) was isolated after chromatography in 4:1 hexane-EtOAc as a colorless oil: $R_{f}$ $0.52\left(2: 1\right.$, h hexane-EtOAc); $[\alpha]_{\mathrm{D}}+46.6\left(c \quad 0.1, \mathrm{CH}_{2} \mathrm{Cl}_{2}\right) ;{ }^{1} \mathrm{H}$ NMR $\left(500 \mathrm{MHz}, \mathrm{CDCl}_{3}, \delta_{\mathrm{H}}\right)$ 8.10-8.06 (m, 2H, Ar), 7.60-7.56 (m, 1H, Ar), 7.47-7.43 (m, 2H, Ar), 7.36-7.22 (m,12H, Ar), 7.06-7.02 (m, 2H, Ar), 5.10 (d, 1H, J=1.9 Hz, H-1), 5.02 (br s, 1H, H-1'), 4.59-4.50 (m, 5H, H-5a, H-5b, $\left.3 \times \mathrm{PhCH}_{2}\right), 4.39\left(\mathrm{~d}, 1 \mathrm{H}, J=12.1 \mathrm{~Hz}, \mathrm{PhCH}_{2}\right), 4.35-4.31(\mathrm{~m}, 1 \mathrm{H}, \mathrm{H}-4)$, 4.18-4.15 (m, 1H, H-4'), $4.12\left(\mathrm{dd}, 1 \mathrm{H}, J_{2^{\prime}, 3^{\prime}}=2.4 \mathrm{~Hz}, J_{2}{ }^{\prime}, 1^{\prime}=0.7 \mathrm{~Hz}, \mathrm{H}-2^{\prime}\right), 4.05-3.99(\mathrm{~m}, 1 \mathrm{H}$, H-3), 3.77 (dd, 1H, $J_{3^{\prime}, 4^{\prime}}=6.1 \mathrm{~Hz}, J_{3^{\prime}, 2^{\prime}}=2.4 \mathrm{~Hz}, \mathrm{H}-3^{\prime}$ ), 3.62-3.50 (m, 3H, H-5a', 5b', H-2), $3.37\left(\mathrm{~s}, 3 \mathrm{H}, \mathrm{OCH}_{3}\right), 2.73$ (br s, $\left.1 \mathrm{H}, \mathrm{OH}\right), 2.25\left(\mathrm{~s}, 3 \mathrm{H}\right.$, tolyl $\left.\mathrm{CH}_{3}\right) ;{ }^{13} \mathrm{C} \mathrm{NMR}\left(500 \mathrm{MHz}, \mathrm{CDCl}_{3}\right.$, $\left.\delta_{\mathrm{C}}\right) 166.5(\underline{\mathrm{C}}=\mathrm{O}), 138.0(\mathrm{Ar}), 137.7(\mathrm{Ar}), 137.6(\mathrm{Ar}) 133.2(\mathrm{Ar}), 131.7(\mathrm{Ar} \times 2), 130.1(\mathrm{Ar} \times$ 2), $129.8(\mathrm{Ar} \times 2), 129.7(\mathrm{Ar}), 128.43(\mathrm{Ar} \times 2), 128.40(\mathrm{Ar} \times 3), 128.39(\mathrm{Ar} \times 2), 127.9(\mathrm{Ar} \times$ 3), $127.8(\mathrm{Ar} \times 2), 127.7(\mathrm{Ar}), 107.8\left(\mathrm{C}-1^{\prime}\right), 107.5$ (C-1), 87.0 (C-2'), 83.6 (C-3'), 82.6 (C-4), 80.7 (C-4'), 76.8 (C-3), $73.4\left(\mathrm{PhCH}_{2}\right), 72.3$ (C-5), 69.5 (C-5'), $64.2\left(\mathrm{PhCH}_{2}\right), 58.7$ (C-2), $55.1\left(\mathrm{OCH}_{3}\right), 21.0\left(\right.$ tolyl $\left.\underline{\mathrm{CH}}_{3}\right)$. HRMS (ESI) calcd $(\mathrm{M}+\mathrm{Na})^{+} \mathrm{C}_{39} \mathrm{H}_{42} \mathrm{O}_{9} \mathrm{~S}: 709.2442$. Found: 709.2445. 


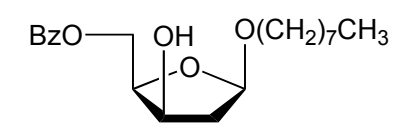

\section{n-Octyl 5-O-benzoyl-2-deoxy- $\beta$-D-threo-pentofuranoside (35)}

Compound (35) was isolated after chromatography in 3:1 hexane-EtOAc as a colorless oil: $R_{f}$ $0.50\left(2: 1\right.$ hexane-EtOAc); $[\alpha]_{\mathrm{D}}-25.2\left(c\right.$ 2.9, $\left.\mathrm{CH}_{2} \mathrm{Cl}_{2}\right) ;{ }^{1} \mathrm{H}$ NMR $\left(500 \mathrm{MHz}, \mathrm{CDCl}_{3}, \delta_{\mathrm{H}}\right)$ 8.07-8.03 (m, 2H, Ar), 7.57-7.53 (m, 1H, Ar), 7.44-7.40 (m, 2H, Ar), 5.20 (d, 1H, $J_{1,2 \mathrm{a}}$ or $\left.J_{1,2 \mathrm{~b}}=4.5 \mathrm{~Hz}, \mathrm{H}-1\right), 4.69\left(\mathrm{dd}, 1 \mathrm{H}, J_{5 \mathrm{a}, 5 \mathrm{~b}}=11.7 \mathrm{~Hz}, J_{5 \mathrm{a}, 4}=4.7 \mathrm{~Hz}, \mathrm{H}-5 \mathrm{a}\right), 4.51\left(\mathrm{dd}, 1 \mathrm{H}, J_{5 \mathrm{~b}, 5 \mathrm{a}}=\right.$ $\left.11.7 \mathrm{~Hz}, J_{5 \mathrm{~b}, 4}=7.3 \mathrm{~Hz}, \mathrm{H}-5 \mathrm{~b}\right), 4.40-4.34(\mathrm{~m}, 1 \mathrm{H}, \mathrm{H}-3), 4.32-4.28$ (m, 1H, H-4), 3.78 (ddd, $1 \mathrm{H}, J=9.5,6.7 \mathrm{~Hz}$, octyl $\left.\mathrm{OCH}_{2}\right), 3.38\left(\mathrm{ddd}, 1 \mathrm{H}, J=9.5,6.7,6.7 \mathrm{~Hz}\right.$, octyl $\left.\mathrm{OCH}_{2}\right), 3.07$ (d, $\left.1 \mathrm{H}, J_{\mathrm{OH}, 3}=11.2 \mathrm{~Hz}, \mathrm{OH}\right), 2.21-2.10(\mathrm{~m}, 2 \mathrm{H}, \mathrm{H}-2 \mathrm{a}, \mathrm{H}-2 \mathrm{~b}), 1.59-1.53\left(\mathrm{~m}, 2 \mathrm{H}\right.$, octyl $\left.\mathrm{CH}_{2}\right)$, $1.34-1.22\left(\mathrm{~m}, 10 \mathrm{H}\right.$, octyl), $0.88\left(\mathrm{t}, 3 \mathrm{H}, J=6.7 \mathrm{~Hz}\right.$, octyl $\left.\mathrm{CH}_{3}\right) ;{ }^{13} \mathrm{C} \mathrm{NMR}\left(500 \mathrm{MHz}, \mathrm{CDCl}_{3}\right.$, $\left.\delta_{\mathrm{C}}\right) 166.4(\underline{\mathrm{C}}=\mathrm{O}), 133.0(\mathrm{Ar}), 130.1(\mathrm{Ar}), 129.7(\mathrm{Ar} \times 2), 128.3(\mathrm{Ar} \times 2), 104.0(\mathrm{C}-1), 82.1$ (C-4), 71.7 (C-3), 67.9 (octyl $\mathrm{OCH}_{2}$ ), 65.0 (C-5), 41.4 (C-2), 31.8 (octyl $\underline{\mathrm{CH}}_{2}$ ), 29.6 (octyl $\underline{\mathrm{CH}}_{2}$ ), 29.3 (octyl $\underline{\mathrm{CH}}_{2}$ ), 29.2 (octyl $\underline{\mathrm{CH}}_{2}$ ), 26.2 (octyl $\underline{\mathrm{CH}}{ }_{2}$ ), 22.6 (octyl $\underline{\mathrm{CH}}_{2}$ ), 14.1 (octyl

$\left.\underline{\mathrm{CH}}_{3}\right) . \quad \mathrm{HRMS}(\mathrm{ESI})$ calcd $(\mathrm{M}+\mathrm{Na})^{+} \mathrm{C}_{20} \mathrm{H}_{36} \mathrm{O}_{5}: 379.2455$. Found: 379.2455.

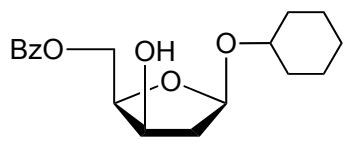

\section{Cyclohexyl 5-O-benzoyl-2-deoxy- $\beta$-D-threo-pentofuranoside (36)}

Compound (36) was isolated after chromatography in 3:1 hexane-EtOAc as a colorless oil: $R_{f}$ $0.45\left(2: 1\right.$ hexane-EtOAc); $[\alpha]_{\mathrm{D}}-54.7\left(c\right.$ 2.2, $\left.\mathrm{CH}_{2} \mathrm{Cl}_{2}\right) ;{ }^{1} \mathrm{H}$ NMR $\left(500 \mathrm{MHz}, \mathrm{CDCl}_{3}, \delta_{\mathrm{H}}\right)$ 8.08-8.06 (m, 2H, Ar), 7.57-7.53 (m, 1H, Ar), 7.43-7.41 (m, 2H, Ar), 5.37 (d, 1H, $J_{1,2 \mathrm{a}}$ or $\left.J_{1,2 \mathrm{~b}}=4.4 \mathrm{~Hz}, \mathrm{H}-1\right), 4.68\left(\mathrm{dd}, 1 \mathrm{H}, J_{5 \mathrm{a}, 5 \mathrm{~b}}=11.7 \mathrm{~Hz}, J_{5 \mathrm{a}, 4}=4.7 \mathrm{~Hz}, \mathrm{H}-5 \mathrm{a}\right), 4.51\left(\mathrm{dd}, 1 \mathrm{H}, J_{5 \mathrm{~b}, 5 \mathrm{a}}=\right.$ 
$\left.11.7 \mathrm{~Hz}, J_{5 \mathrm{~b}, 4}=7.3 \mathrm{~Hz}, \mathrm{H}-5 \mathrm{~b}\right), 4.39-4.34(\mathrm{~m}, 1 \mathrm{H}, \mathrm{H}-3), 4.28\left(\mathrm{ddd}, 1 \mathrm{H}, J_{4,5 \mathrm{~b}}=7.3 \mathrm{~Hz}, J_{4,5 \mathrm{a}}=\right.$ $\left.4.7 \mathrm{~Hz}, J_{4,3}=4.3 \mathrm{~Hz}, \mathrm{H}-4\right), 3.71-3.66(\mathrm{~m}, 1 \mathrm{H}$, cyclohexyl OCH$), 3.22(\mathrm{~d}, 1 \mathrm{H}, J=11.3 \mathrm{~Hz}$, OH), 2.18-2.08 (m, 2H, H-2a, H-2b), 1.91-1.86 (m, 2H, cyclohexyl), 1.53-1.50 (m, 1H, cyclohexyl), 1.41-1.17 (m, 5H, cyclohexyl); ${ }^{13} \mathrm{C} \mathrm{NMR}\left(500 \mathrm{MHz}, \mathrm{CDCl}_{3}, \delta_{\mathrm{C}}\right) 166.4(\underline{\mathrm{C}}=\mathrm{O})$, $132.9(\mathrm{Ar}), 130.2(\mathrm{Ar}), 129.7(\mathrm{Ar} \times 2), 128.3(\mathrm{Ar} \times 2), 101.7(\mathrm{C}-1), 82.0(\mathrm{C}-4), 75.0$ (cyclohexyl $\mathrm{O} \underline{\mathrm{CH}}$ ), 71.8 (C-3), 65.0 (C-5), 41.6 (C-2), 33.9 (cyclohexyl $\underline{\mathrm{CH}}_{2}$ ), 31.4 (cyclohexyl $\underline{\mathrm{CH}}_{2}$ ), 25.6 (cyclohexyl $\left.\underline{\mathrm{CH}}_{2}\right), 24.1$ (cyclohexyl $\left.\underline{\mathrm{CH}}_{2}\right), 23.8$ (cyclohexyl $\left.\underline{\mathrm{CH}}_{2}\right)$. HRMS (ESI) calcd $(\mathrm{M}+\mathrm{Na})^{+} \mathrm{C}_{18} \mathrm{H}_{24} \mathrm{O}_{5}: 343.1516$. Found: 343.1516.

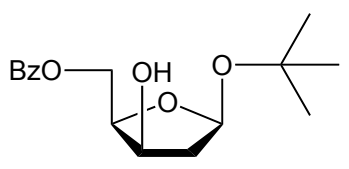

\section{t-Butyl 5-O-benzoyl-2-deoxy- $\beta$-D-threo-pentofuranoside (37)}

Compound (37) was isolated after chromatography in 4:1 hexane-EtOAc as a colorless oil: $R_{f}$ $0.43\left(2: 1\right.$ hexane-EtOAc); $[\alpha]_{\mathrm{D}}-63.5\left(c \quad 0.2, \mathrm{CH}_{2} \mathrm{Cl}_{2}\right) ;{ }^{1} \mathrm{H}$ NMR $\left(500 \mathrm{MHz}, \mathrm{CDCl}_{3}, \delta_{\mathrm{H}}\right)$ 8.09-8.06 (m, 2H, Ar), 7.57-7.53 (m, 1H, Ar), 7.45-7.41 (m, 2H, Ar), $5.52\left(\mathrm{dd}, 1 \mathrm{H}, J_{1,2 \mathrm{a}}=\right.$ $\left.3.4 \mathrm{~Hz}, J_{1,2 \mathrm{~b}}=1.6 \mathrm{~Hz}, \mathrm{H}-1\right), 4.69\left(\mathrm{dd}, 1 \mathrm{H}, J_{5 \mathrm{a}, 5 \mathrm{~b}}=11.5 \mathrm{~Hz}, J_{5 \mathrm{a}, 4}=5.2 \mathrm{~Hz}, \mathrm{H}-5 \mathrm{a}\right), 4.56(\mathrm{dd}, 1 \mathrm{H}$, $\left.J_{5 \mathrm{a}, 5 \mathrm{~b}}=11.5 \mathrm{~Hz}, J_{5 \mathrm{~b}, 4}=7.3 \mathrm{~Hz}, \mathrm{H}-5 \mathrm{~b}\right), 4.40-4.33(\mathrm{~m}, 1 \mathrm{H}, \mathrm{H}-3), 4.23\left(\mathrm{ddd}, 1 \mathrm{H}, J_{4,5 \mathrm{~b}}=7.3 \mathrm{~Hz}\right.$, $\left.J_{4,5 \mathrm{a}}=5.2, J_{4,3}=3.9 \mathrm{~Hz}, \mathrm{H}-4\right), 3.31\left(\mathrm{~d}, 1 \mathrm{H}, J_{\mathrm{OH}, 3}=11.4 \mathrm{~Hz}, \mathrm{OH}\right), 2.11-2.10(\mathrm{~m}, 2 \mathrm{H}, \mathrm{H}-2 \mathrm{a}$, $\mathrm{H}-2 \mathrm{~b}), 1.26\left(\mathrm{~s}, 9 \mathrm{H}, \mathrm{C}\left(\mathrm{CH}_{3}\right)_{3}\right) ;{ }^{13} \mathrm{C} \mathrm{NMR}\left(500 \mathrm{MHz}, \mathrm{CDCl}_{3}, \delta_{\mathrm{C}}\right) 166.5(\underline{\mathrm{C}}=\mathrm{O}), 132.9$ (Ar), $130.1(\mathrm{Ar}), 129.8(\mathrm{Ar} \times 2), 128.4(\mathrm{Ar} \times 2), 98.9(\mathrm{C}-1), 81.9(\mathrm{C}-4), 75.5\left(\mathrm{OC}\left(\mathrm{CH}_{3}\right)_{3}\right), 72.1$ (C-3), 65.0 (C-5), $42.1(\mathrm{C}-2), 29.0\left(\mathrm{C}\left(\underline{\mathrm{CH}}_{3}\right)_{3}\right)$. HRMS (ESI) calcd $(\mathrm{M}+\mathrm{Na})^{+} \mathrm{C}_{16} \mathrm{H}_{22} \mathrm{O}_{5}$ : 317.1359. Found: 317.1355. 


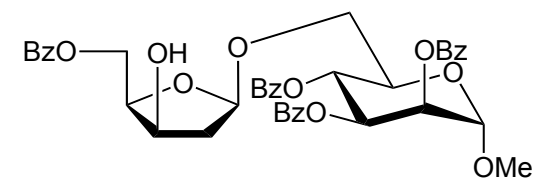

Methyl 5-O-(5-O-benzoyl-2-deoxy-2- $\beta$-D-threo-pentofuranosyl)-2,3,4-tri- $O$-benzoyl- $\alpha$-Dmannopyranoside (38)

Compound (38) was isolated after chromatography in 4:1 hexane-EtOAc as a colorless oil: $R_{f}$ $0.21\left(2: 1\right.$ hexane-EtOAc); $[\alpha]_{\mathrm{D}}-84.8\left(c\right.$ 3.8, $\left.\mathrm{CH}_{2} \mathrm{Cl}_{2}\right) ;{ }^{1} \mathrm{H}$ NMR $\left(500 \mathrm{MHz}, \mathrm{CDCl}_{3}, \delta_{\mathrm{H}}\right)$ 8.13-8.09 (m, 2H, Ar), 8.05-8.01 (m, 2H, Ar), 7.97-7.94 (m, 2H, Ar), 7.84-7.80 (m, 2H, Ar), 7.63-7.34 (m, 10H, Ar), 7.28-7.23 (m, 2H, Ar), $5.95\left(\mathrm{dd}, 1 \mathrm{H}, J_{4^{\prime}, 5^{\prime}}=10.0 \mathrm{~Hz}, J_{4^{\prime}, 3^{\prime}}=10.0 \mathrm{~Hz}\right.$, H-4'), $5.86\left(\mathrm{dd}, 1 \mathrm{H}, J_{3^{\prime}, 4^{\prime}}=10.0 \mathrm{~Hz}, J_{3^{\prime}, 2^{\prime}}=3.3 \mathrm{~Hz}, \mathrm{H}-3^{\prime}\right), 5.70\left(\mathrm{dd}, 1 \mathrm{H}, J_{2^{\prime}, 3^{\prime}}=3.3 \mathrm{~Hz}, J_{2^{\prime}, 1^{\prime}}=\right.$ $\left.1.9 \mathrm{~Hz}, \mathrm{H}-2^{\prime}\right), 5.28$ (d, 1H, $\left.J_{1,2}=4.4 \mathrm{~Hz}, \mathrm{H}-1\right), 4.99$ (d, 1H, $\left.J_{1^{\prime}, 2^{\prime}}=1.9 \mathrm{~Hz}, \mathrm{H}-1^{\prime}\right), 4.68$ (dd, $\left.1 \mathrm{H}, J_{5 \mathrm{a}, 5 \mathrm{~b}}=11.8 \mathrm{~Hz}, J_{5 \mathrm{a}, 4}=4.4 \mathrm{~Hz}, \mathrm{H}-5 \mathrm{a}\right), 4.60\left(\mathrm{dd}, 1 \mathrm{H}, J_{5 \mathrm{~b}, 5 \mathrm{a}}=11.8 \mathrm{~Hz}, J_{5 \mathrm{~b}, 4}=7.6 \mathrm{~Hz}, \mathrm{H}-5 \mathrm{~b}\right)$, 4.44-4.38 (m, 1H, H-3), 4.37-4.32 (m, 1H, H-4), 4.22 (ddd, $1 \mathrm{H}, J_{5^{\prime}, 4}{ }^{\prime}=10.0 \mathrm{~Hz}, J_{5^{\prime}, 6 \mathrm{~b}^{\prime}}=4.0$ $\left.\mathrm{Hz}, J_{5^{\prime}, 6 \mathrm{a}^{\prime}}=2.6 \mathrm{~Hz}, \mathrm{H}-5^{\prime}\right), 4.12\left(\mathrm{dd}, 1 \mathrm{H}, J_{6 \mathrm{a}^{\prime}, 6 \mathrm{~b}^{\prime}}=11.0 \mathrm{~Hz}, J_{6 \mathrm{a}^{\prime}, 5}=2.6 \mathrm{~Hz}, \mathrm{H}-6 \mathrm{a}^{\prime}\right), 3.61(\mathrm{dd}, 1 \mathrm{H}$, $\left.J_{6 \mathrm{~b}^{\prime}, 6 \mathrm{a}}{ }^{\prime}=11.0 \mathrm{~Hz}, J_{6 \mathrm{~b}^{\prime}, 5}{ }^{\prime}=4.0 \mathrm{~Hz}, \mathrm{H}^{-6 \mathrm{~b}^{\prime}}\right), 3.55\left(\mathrm{~s}, 3 \mathrm{H}, \mathrm{OCH}_{3}\right), 3.31\left(\mathrm{~d}, 1 \mathrm{H}, J_{\mathrm{OH}, 3}=10.9 \mathrm{~Hz}\right.$, $\mathrm{OH}), 2.37-2.31(\mathrm{~m}, 1 \mathrm{H}, \mathrm{H}-2 \mathrm{a}), 2.20-2.14(\mathrm{~m}, 1 \mathrm{H}, \mathrm{H}-2 \mathrm{~b}) ;{ }^{13} \mathrm{C} \mathrm{NMR}\left(500 \mathrm{MHz}, \mathrm{CDCl}_{3}, \delta_{\mathrm{C}}\right)$ $166.4(\underline{\mathrm{C}}=\mathrm{O}), 165.5(\underline{\mathrm{C}}=\mathrm{O}), 165.44(\underline{\mathrm{C}}=\mathrm{O}), 165.42(\underline{\mathrm{C}}=\mathrm{O}), 133.42$ (Ar), 133.39 (Ar) 133.0 (Ar), 132.9 (Ar), 130.1 (Ar), $130.0(\mathrm{Ar} \times 2), 129.74(\mathrm{Ar} \times 2), 129.73(\mathrm{Ar} \times 2), 129.69(\mathrm{Ar} \times$ 2), $129.3(\mathrm{Ar}), 129.2(\mathrm{Ar}), 129.1(\mathrm{Ar}), 128.5(\mathrm{Ar} \times 2), 128.4(\mathrm{Ar} \times 2), 128.3(\mathrm{Ar} \times 2), 128.2$ $(\mathrm{Ar} \times 2), 104.1(\mathrm{C}-1), 98.8\left(\mathrm{C}-1^{\prime}\right), 82.6(\mathrm{C}-4), 71.5(\mathrm{C}-3), 70.2\left(\mathrm{C}-2^{\prime}\right.$ or $\mathrm{C}-3$ '), $69.5\left(\mathrm{C}-5^{\prime}\right)$, 66.9 (C-4'), 64.9 (C-5), 64.7 (C-6'), $55.6\left(\mathrm{OCH}_{3}\right), 41.7$ (C-2). $\quad$ HRMS (ESI) calcd (M+Na) ${ }^{+}$ $\mathrm{C}_{40} \mathrm{H}_{38} \mathrm{O}_{13}: 749.2205$. Found: 749.2192. 


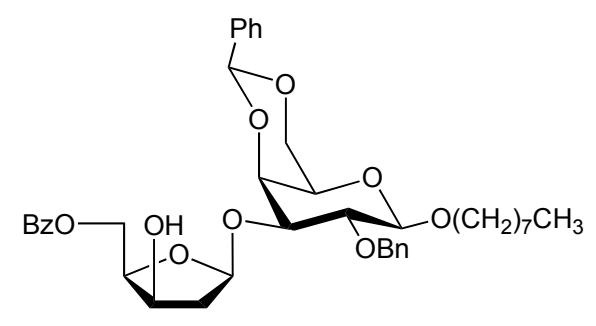

n-Octyl 3-O-(5-O-benzoyl-2-deoxy- $\beta$-D-threo-pentofuranosyl)-2- $O$-benzyl-4,6- $O$-benzylidene- $\beta$-D-galactopyranoside (39)

Compound (39) was isolated after chromatography in 4:1 hexane-EtOAc as a colorless oil: $R_{\mathrm{f}}$ $0.44\left(2: 1\right.$ hexane-EtOAc); $[\alpha]_{\mathrm{D}}+18.1\left(c \quad 0.6, \mathrm{CH}_{2} \mathrm{Cl}_{2}\right) ;{ }^{1} \mathrm{H}$ NMR $\left(500 \mathrm{MHz}, \mathrm{CDCl}_{3}, \delta_{\mathrm{H}}\right)$ 8.10-8.07 (m, 2H, Ar), 7.60-7.57 (m, 3H, Ar), 7.48-7.44 (m, 2H, Ar), 7.38-7.26 (m, 8H, Ar), $5.76(\mathrm{~s}, 1 \mathrm{H}, \mathrm{PhCH}), 5.45\left(\mathrm{~d}, 1 \mathrm{H}, J_{1,2 \mathrm{a}}\right.$ or $\left.J_{1,2 \mathrm{~b}}=4.4 \mathrm{~Hz}, \mathrm{H}-1\right), 4.90(\mathrm{~d}, 1 \mathrm{H}, J=11.3 \mathrm{~Hz}$, $\left.\mathrm{PhCH}_{2}\right), 4.75\left(\mathrm{dd}, 1 \mathrm{H}, J_{5 \mathrm{a}, 5 \mathrm{~b}}=11.9 \mathrm{~Hz}, J_{5 \mathrm{a}, 4}=8.0 \mathrm{~Hz}, \mathrm{H}-5 \mathrm{a}\right), 4.63(\mathrm{~d}, 1 \mathrm{H}, J=11.3 \mathrm{~Hz}$, $\left.\mathrm{PhCH}_{2}\right), 4.51\left(\mathrm{dd}, 1 \mathrm{H}, J_{5 \mathrm{~b}, 5 \mathrm{a}}=11.9 \mathrm{~Hz}, J_{5 \mathrm{~b}, 4}=3.6 \mathrm{~Hz}, \mathrm{H}-5 \mathrm{~b}\right), 4.50-4.46\left(\mathrm{~m}, 1 \mathrm{H}, \mathrm{H}-4^{\prime}\right), 4.38$ $\left(\mathrm{d}, 1 \mathrm{H}, J_{1^{\prime}, 2^{\prime}}=7.6 \mathrm{~Hz}, \mathrm{H}-1^{\prime}\right), 4.36-4.29\left(\mathrm{~m}, 3 \mathrm{H}, \mathrm{H}-3, \mathrm{H}-4, \mathrm{H}-6 \mathrm{a}^{\prime}\right), 4.10$ (dd, 1H, $J_{6 \mathrm{~b}^{\prime}, 6 \mathrm{a}^{\prime}}=$ $\left.12.3 \mathrm{~Hz}, J_{6 \mathrm{~b}^{\prime}, 5}{ }^{\prime}=1.7 \mathrm{~Hz}, \mathrm{H}-6 \mathrm{~b} '\right), 3.99$ (ddd, $1 \mathrm{H}, J=9.4,6.5,6.5 \mathrm{~Hz}$, octyl $\left.\mathrm{OCH}_{2}\right), 3.78-3.63$ (m, 2H, H-2', H-3'), 3.54-3.45 (m, 2H, octyl $\left.\mathrm{OCH}_{2}, \mathrm{OH}\right), 3.43-3.41\left(\mathrm{~m}, 1 \mathrm{H}, \mathrm{H}-5^{\prime}\right)$, 2.25-2.20 (m, 1H, H-2a), 2.10-2.04 (m, 1H, H-2b), 1.69-1.59 (m, 2H, octyl $\mathrm{CH}_{2}$ ), 1.42-1.26 (m, 11H, octyl), $0.87\left(\mathrm{t}, 3 \mathrm{H}, J=6.80 \mathrm{~Hz}\right.$, octyl $\left.\mathrm{CH}_{3}\right) ;{ }^{13} \mathrm{C} \mathrm{NMR}\left(500 \mathrm{MHz}, \mathrm{CDCl}_{3}, \delta_{\mathrm{C}}\right) 166.4$ $(\underline{\mathrm{C}}=\mathrm{O}), 138.9(\mathrm{Ar}), 137.8(\mathrm{Ar}), 133.09(\mathrm{Ar}), 130.06(\mathrm{Ar}), 129.7(\mathrm{Ar} \times 2), 128.9(\mathrm{Ar}), 128.4$ $(\mathrm{Ar} \times 2), 128.3(\mathrm{Ar} \times 2), 128.1(\mathrm{Ar} \times 2), 127.8(\mathrm{Ar} \times 2), 127.6(\mathrm{Ar}), 126.3(\mathrm{Ar} \times 2), 106.3$ (C-1), 103.6 (C-1'), 101.0 (PhCH $), 82.7$ ((C-4), 78.7 (C-2’ or C-3), 78.1 (C-3 or C-2'), 75.9 (C-4'), $75.2\left(\mathrm{Ph}^{\mathrm{C}} \mathrm{H}_{2}\right), 71.6$ (C-3'), 70.0 (octyl $\left.\mathrm{O}^{\mathrm{C}} \mathrm{H}_{2}\right), 69.1$ (C-6'), 66.4 (C-5'), 65.1 (C-5), 41.4 (C-2), 31.8 (octyl $\underline{\mathrm{CH}}_{2}$ ), 29.7 (octyl $\underline{\mathrm{CH}}_{2}$ ), 29.4 (octyl $\underline{\mathrm{CH}}_{2}$ ), 29.3 (octyl $\underline{\mathrm{CH}}_{2}$ ), 26.2 (octyl 
$\underline{\mathrm{CH}}_{2}$ ), 22.7 (octyl $\left.\underline{\mathrm{CH}}_{2}\right), 14.1$ (octyl $\left.\underline{\mathrm{CH}}_{3}\right) . \quad \mathrm{HRMS}(\mathrm{ESI})$ calcd $(\mathrm{M}+\mathrm{Na})^{+} \mathrm{C}_{40} \mathrm{H}_{50} \mathrm{O}_{10}$ : 713.3296. Found: 713.3300.

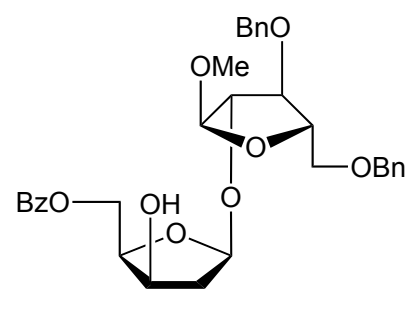

Methyl 2-O-(5-O-benzoyl-2-deoxy- $\beta$-D-threo-pentofuranosyl)-3,4-di- $O$-benzyl- $\alpha$-Darabinofuranoside (40)

Compound (40) was isolated after chromatography in 3:1 hexane-EtOAc as a colorless oil: $R_{\mathrm{f}}$ $0.32\left(2: 1\right.$ hexane-EtOAc); $[\alpha]_{\mathrm{D}}+41.5\left(c \quad 0.2, \mathrm{CH}_{2} \mathrm{Cl}_{2}\right) ;{ }^{1} \mathrm{H}$ NMR $\left(500 \mathrm{MHz}, \mathrm{CDCl}_{3}, \delta_{\mathrm{H}}\right)$ 8.04-8.00 (m, 2H, Ar), 7.61-7.56 (m, 1H, Ar), 7.48-7.43 (m, 2H, Ar), 7.36-7.26 (m, 10H, Ar), $5.38\left(\mathrm{~d}, 1 \mathrm{H}, J_{1,2 \mathrm{a}}\right.$ or $\left.J_{1,2 \mathrm{~b}}=4.3 \mathrm{~Hz}, \mathrm{H}-1\right), 4.95(\mathrm{~s}, 1 \mathrm{H}, \mathrm{H}-1$ '), $4.76(\mathrm{~d}, 1 \mathrm{H}, J=11.9 \mathrm{~Hz}$, $\left.\mathrm{PhCH}_{2}\right), 4.73\left(\mathrm{dd}, 1 \mathrm{H}, J_{5 \mathrm{a}, 5 \mathrm{~b}}=11.7 \mathrm{~Hz}, J_{5 \mathrm{a}, 4}=4.7 \mathrm{~Hz}, \mathrm{H}-5 \mathrm{a}\right), 4.63-4.49(\mathrm{~m}, 4 \mathrm{H}, \mathrm{H}-5 \mathrm{~b}, 3 \times$ $\mathrm{PhCH}_{2}$ ), 4.40-4.35 (m, 1H, H-3), 4.33-4.27 (m, 2H, H-2', H-4), 4.20-4.15 (m, 1H, H-4'), 4.09-4.06 (m, 1H, H-3'), 3.61 (dd, $\left.1 \mathrm{H}, J_{5 \mathrm{a}^{\prime}, 5 \mathrm{~b}^{\prime}}=10.8 \mathrm{~Hz}, J_{5 \mathrm{a}^{\prime}, 4^{\prime}}=3.2 \mathrm{~Hz}, \mathrm{H}-5 \mathrm{a}^{\prime}\right), 3.53$ (dd, $1 \mathrm{H}$, $\left.J_{5 \mathrm{~b}^{\prime}, 5 \mathrm{a}^{\prime}}=10.8 \mathrm{~Hz}, J_{5 \mathrm{~b}^{\prime}, 4^{\prime}}=4.6 \mathrm{~Hz}, \mathrm{H}-5 \mathrm{~b}^{\prime}\right), 3.39\left(\mathrm{~s}, 3 \mathrm{H}, \mathrm{OCH}_{3}\right), 2.87$ (br s, 1H, OH), 2.26-2.15 (m, 2H, H-2a, H-2b); ${ }^{13} \mathrm{C}$ NMR (500 MHz, $\left.\mathrm{CDCl}_{3}, \delta_{\mathrm{C}}\right) 166.4(\underline{\mathrm{C}}=\mathrm{O}), 137.92(\mathrm{Ar}), 137.88$ (Ar), 133.1 (Ar), 130.0 (Ar), 129.8 ( $\mathrm{Ar} \times 2), 128.5(\mathrm{Ar} \times 2), 128.44(\mathrm{Ar} \times 2), 128.41(\mathrm{Ar} \times 2)$, 128.38 $(\mathrm{Ar} \times 2), 128.0(\mathrm{Ar} \times 2), 127.7(\mathrm{Ar} \times 2), 107.5\left(\mathrm{C}-1^{\prime}\right), 104.5(\mathrm{C}-1), 86.0\left(\mathrm{C}-2^{\prime}\right), 84.3$ (C-3'), 82.7 (C-4), 82.0 (C-4'), $73.5\left(\mathrm{PhCH}_{2}\right), 72.5\left(\mathrm{PhCH}_{2}\right), 71.3$ (C-3), 69.4 (C-5'), 64.6 (C-5), $54.9\left(\mathrm{O}_{\underline{C H}}\right), 41.6(\mathrm{C}-2)$. $\quad \mathrm{HRMS}(\mathrm{ESI})$ calcd $(\mathrm{M}+\mathrm{Na})^{+} \mathrm{C}_{32} \mathrm{H}_{36} \mathrm{O}_{9}$ : 587.2252. Found: 587.2254 


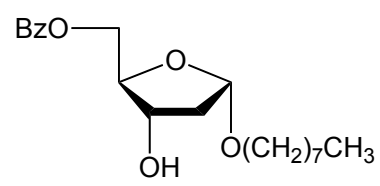

\section{n-Octyl 5-O-benzoyl-2-deoxy- $\alpha$-D-erythro-pentofuranoside (41)}

Compound (41) was isolated after chromatography in 5:1 hexane-EtOAc as a colorless oil: $R_{\mathrm{f}}$ $0.57\left(2: 1\right.$ hexane-EtOAc); $[\alpha]_{\mathrm{D}}+52.4\left(c \quad 0.1, \mathrm{CH}_{2} \mathrm{Cl}_{2}\right) ;{ }^{1} \mathrm{H}$ NMR $\left(500 \mathrm{MHz}, \mathrm{CDCl}_{3}, \delta_{\mathrm{H}}\right)$ 8.04-8.01 (m, 2H, Ar), 7.59-7.56 (m, 1H, Ar), 7.47-7.42 (m, 2H, Ar), $5.24(\mathrm{~d}, 1 \mathrm{H}, J=4.3$ Hz, H-1), 4.43-4.31 (m, 3H, H-4, H-5a, H-5b), 4.27-4.22 (m, 1H, H-3), 3.77 (ddd, 1H, J= 9.6, 6.7, 6.7 Hz, octyl $\left.\mathrm{OCH}_{2}\right), 3.42\left(\mathrm{ddd}, 1 \mathrm{H}, J=9.6,6.7,6.7 \mathrm{~Hz}\right.$, octyl $\left.\mathrm{OCH}_{2}\right), 3.00($ br s, $1 \mathrm{H}$, $\mathrm{OH}), 2.18-2.07$ (m, 2H, H-2a, H-2b), 1.61-1.55 (m, 2H, octyl $\left.\mathrm{CH}_{2}\right), 1.36-1.23$ (m, 10H, octyl $\left.\mathrm{CH}_{2}\right), 0.88\left(\mathrm{t}, 3 \mathrm{H}, J=6.9 \mathrm{~Hz}\right.$, octyl $\left.\mathrm{CH}_{3}\right) ;{ }^{13} \mathrm{C} \mathrm{NMR}\left(500 \mathrm{MHz}, \mathrm{CDCl}_{3}, \delta_{\mathrm{C}}\right) 166.3$ $(\underline{\mathrm{C}}=\mathrm{O}), 133.2(\mathrm{Ar}), 129.8(\mathrm{Ar}), 129.6(\mathrm{Ar} \times 2), 128.4(\mathrm{Ar} \times 2), 104.3(\mathrm{C}-1), 85.2(\mathrm{C}-4), 73.3$ (C-3), 67.8 (octyl $\mathrm{OCH}_{2}$ ), 64.7 (C-5), 40.9 (C-2), 31.8 (octyl $\underline{\mathrm{CH}}_{2}$ ), 29.6 (octyl $\underline{\mathrm{CH}}_{2}$ ), 29.3 (octyl $\underline{\mathrm{CH}}_{2}$ ), 29.2 (octyl $\underline{\mathrm{CH}}_{2}$ ), 26.2 (octyl $\left.\underline{\mathrm{C}} \mathrm{H}_{2}\right), 22.6$ (octyl $\left.\underline{\mathrm{CH}}_{2}\right), 14.1$ (octyl $\left.\underline{\mathrm{C}} \mathrm{H}_{3}\right)$. HRMS (ESI) calcd $(\mathrm{M}+\mathrm{Na})^{+} \mathrm{C}_{20} \mathrm{H}_{30} \mathrm{O}_{5}$ : 373.1986. Found: 373.1991.

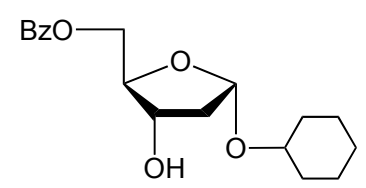

\section{Cyclohexyl 5-O-benzoyl-2-deoxy- $\alpha$-D-erythro-pentofuranoside (42)}

Compound (42) was isolated after chromatography in 4:1 hexane-EtOAc as a colorless oil: $R_{\mathrm{f}}$ $0.51\left(2: 1\right.$ hexane-EtOAc); $[\alpha]_{\mathrm{D}}+21.1\left(c\right.$ 0.03, $\left.\mathrm{CH}_{2} \mathrm{Cl}_{2}\right) ;{ }^{1} \mathrm{H} \mathrm{NMR}\left(500 \mathrm{MHz}, \mathrm{CDCl}_{3}, \delta_{\mathrm{H}}\right)$ 8.04-8.00 (m, 2H, Ar), 7.59-7.55 (m, 1H, Ar), 7.47-7.42 (m, 2H, Ar), $5.43\left(\mathrm{~d}, 1 \mathrm{H}, J_{1,2 \mathrm{a}}\right.$ or $\left.J_{1,2 \mathrm{~b}}=4.3 \mathrm{~Hz}, \mathrm{H}-1\right), 4.44-4.30$ (m, 3H, H-4, H-5a, H-5b), 4.27-4.23 (m, 1H, H-3), 3.72-3.63 
(m, 1H, cyclohexyl OCH), 3.20 (br, 1H, OH), 2.17-2.04 (m, 2H, H-2a, H-2b), 1.91-1.86 (m, $1 \mathrm{H}$, cyclohexyl), 1.73-1.71 (m, 2H, cyclohexyl), 1.55-1.52 (m, 1H, cyclohexyl), 1.43-1.19 (m, 5H, cyclohexyl); ${ }^{13} \mathrm{C}$ NMR $\left(500 \mathrm{MHz}, \mathrm{CDCl}_{3}, \delta_{\mathrm{C}}\right) 166.3(\mathrm{C}=\mathrm{O}), 133.1(\mathrm{Ar}), 129.8(\mathrm{Ar})$, 129.6 $(\mathrm{Ar} \times 2), 128.4(\mathrm{Ar} \times 2), 102.2(\mathrm{C}-1), 85.2(\mathrm{C}-4), 74.8($ cyclohexyl OC$H), 73.5(\mathrm{C}-3)$, 64.7 (C-5), 41.1 (C-2), 33.8 (cyclohexyl $\left.\underline{\mathrm{CH}}_{2}\right), 31.5$ (cyclohexyl $\left.\underline{\mathrm{CH}}_{2}\right), 25.6$ (cyclohexyl $\left.\underline{\mathrm{CH}}_{2}\right)$, 24.1 (cyclohexyl $\underline{\mathrm{CH}}_{2}$ ), 23.9 (cyclohexyl $\left.\underline{\mathrm{CH}}_{2}\right)$. HRMS (ESI) calcd $(\mathrm{M}+\mathrm{Na})^{+} \mathrm{C}_{18} \mathrm{H}_{24} \mathrm{O}_{5}$ : 343.1516. Found: 343.1518.

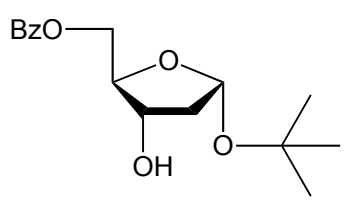

\section{t-Butyl 5-O-bezoyl-2-deoxy- $\alpha$-D-erythro-pentofuranoside (43)}

Compound (43) was isolated after chromatography in 6:1 hexane-EtOAc as a colorless oil: $R_{f}$ $0.45\left(2: 1\right.$ hexane-EtOAc); $[\alpha]_{\mathrm{D}}+81.6\left(c \quad 0.1, \mathrm{CH}_{2} \mathrm{Cl}_{2}\right) ;{ }^{1} \mathrm{H}$ NMR $\left(500 \mathrm{MHz}, \mathrm{CDCl}_{3}, \delta_{\mathrm{H}}\right)$ 8.03-8.01 (m, 2H, Ar), 7.59-7.55 (m, 1H, Ar), 7.46-7.43 (m, 2H, Ar), $5.60\left(\mathrm{~d}, 1 \mathrm{H}, J_{1,2 \mathrm{a}}\right.$ or $\left.J_{1,2 \mathrm{~b}}=4.5 \mathrm{~Hz}, \mathrm{H}-1\right), 4.46-4.23(\mathrm{~m}, 1 \mathrm{H}, \mathrm{H}-4), 4.37\left(\mathrm{dd}, 1 \mathrm{H}, J_{5 \mathrm{a}, 5 \mathrm{~b}}=11.6 \mathrm{~Hz}, J_{5 \mathrm{a}, 4}=5.3 \mathrm{~Hz}\right.$ H-5a), $4.28\left(\mathrm{dd}, 1 \mathrm{H}, J_{5 \mathrm{~b}, 5 \mathrm{a}}=11.6 \mathrm{~Hz}, J_{5 \mathrm{~b}, 4}=5.2 \mathrm{~Hz}, \mathrm{H}-5 \mathrm{~b}\right), 4.26-4.22(\mathrm{~m}, 1 \mathrm{H}, \mathrm{H}-3), 3.25$ (br, 1H, OH), 2.18-1.98 (m, 2H, H-2a, H-2b), 1.24 (s, 9H, C(CH $\left.\mathrm{CH}_{3}\right) ;{ }^{13} \mathrm{C}$ NMR (500 MHz, $\mathrm{CDCl}_{3}$, $\left.\delta_{\mathrm{C}}\right) 166.3(\underline{\mathrm{C}}=\mathrm{O}), 133.1(\mathrm{Ar}), 129.9(\mathrm{Ar}), 129.6(\mathrm{Ar} \times 2), 128.4(\mathrm{Ar} \times 2), 99.5(\mathrm{C}-1), 84.8$

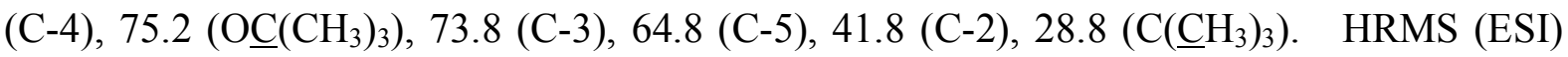
calcd $(\mathrm{M}+\mathrm{Na})^{+} \mathrm{C}_{16} \mathrm{H}_{22} \mathrm{O}_{5}: 317.1359$ found 317.1362 . 


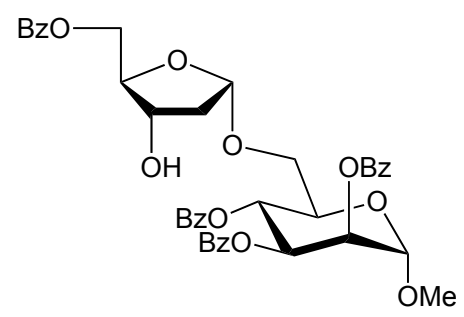

Methyl 5-O-(5-O-benzoyl-2-deoxy- $\alpha$-D-erythro-pentofuranosyl)-2,3,4-tri-O-benzoyl- $\alpha$-Dmannopyranoside (44)

Compound (44) was isolated after chromatography in 4:1 hexane-EtOAc as a colorless oil: $R_{f}$ $0.23\left(2: 1\right.$ hexane-EtOAc); $[\alpha]_{\mathrm{D}}-59.9\left(c \quad 0.3, \mathrm{CH}_{2} \mathrm{Cl}_{2}\right) ;{ }^{1} \mathrm{H}$ NMR $\left(500 \mathrm{MHz}, \mathrm{CDCl}_{3}, \delta_{\mathrm{H}}\right)$ 8.16-8.12 (m, 2H, Ar), 8.01-7.96 (m, 4H, Ar), 7.83-7.80 (m, 2H, Ar), 7.63-7.48 (m, 5H, Ar), 7.45-7.36 (m, 5H, Ar), 7.25-7.23 (m, 2H, Ar), $6.11\left(\mathrm{dd}, 1 \mathrm{H}, J_{4^{\prime}, 5^{\prime}}=10.2 \mathrm{~Hz}, J_{4}{ }^{,} 3^{\prime}=10.2 \mathrm{~Hz}\right.$, H-4'), $5.89\left(\mathrm{dd}, 1 \mathrm{H}, J_{3^{\prime}, 4^{\prime}}=10.2 \mathrm{~Hz}, J_{3^{\prime}, 2^{\prime}}=3.3 \mathrm{~Hz}, \mathrm{H}-3^{\prime}\right), 5.67\left(\mathrm{dd}, 1 \mathrm{H}, J_{2^{\prime}, 3^{\prime}}=3.3 \mathrm{~Hz}, J_{2}{ }^{\prime}, 1^{\prime}=\right.$ $\left.1.9 \mathrm{~Hz}, \mathrm{H}-2^{\prime}\right), 5.32$ (d, 1H, $\left.J_{1,2}=3.8 \mathrm{~Hz}, \mathrm{H}-1\right), 5.01$ (d, $\left.1 \mathrm{H}, J_{1^{\prime}, 2^{\prime}}=1.9 \mathrm{~Hz}, \mathrm{H}-1^{\prime}\right), 4.44-4.39$ (m, 1H, H-4), 4.38-4.29 (m, 3H, H-5a, H-5b, H-3), 4.23-4.17 (m, 1H, H-5'), 4.02 (dd, 1H, $\left.J_{6 \mathrm{a}^{\prime}, 6 \mathrm{~b}^{\prime}}=11.1 \mathrm{~Hz}, J_{6 \mathrm{a}^{\prime}, 5^{\prime}}=3.6 \mathrm{~Hz}, \mathrm{H}-6 \mathrm{a}^{\prime}\right), 3.63\left(\mathrm{dd}, 1 \mathrm{H}, J_{6 \mathrm{~b}^{\prime}, 6 \mathrm{a}^{\prime}}=11.1 \mathrm{~Hz}, J_{6 \mathrm{~b}^{\prime}, 5^{\prime}}=2.3 \mathrm{~Hz}\right.$, H-6b'), 3.53 (s, 3H, OCH $), 2.28-2.16$ (m, 2H, H-2a, H-2b); ${ }^{13} \mathrm{C}$ NMR (500 MHz, $\left.\mathrm{CDCl}_{3}, \delta_{\mathrm{C}}\right)$ $166.3(\underline{\mathrm{C}}=\mathrm{O}), 165.8(\underline{\mathrm{C}}=\mathrm{O}), 165.5(\underline{\mathrm{C}}=\mathrm{O} \times 2), 133.6(\operatorname{Ar}), 133.5(\operatorname{Ar}), 133.1(\operatorname{Ar} \times 2), 130.0$ $(\mathrm{Ar} \times 2), 129.84(\mathrm{Ar} \times 2), 129.76(\mathrm{Ar}), 129.7(\mathrm{Ar} \times 2), 129.6(\mathrm{Ar} \times 2), 129.3(\mathrm{Ar}), 129.1(\mathrm{Ar})$ $129.0(\mathrm{Ar}), 128.7(\mathrm{Ar} \times 2), 128.5(\mathrm{Ar} \times 2), 128.4(\mathrm{Ar} \times 2), 128.3(\mathrm{Ar} \times 2), 104.8(\mathrm{C}-1), 98.7$ (C-1'), 85.3 (C-4), 73.1 (C-3), 70.4 (C-2’), 69.9 (C-3'), 69.5 (C-5'), 66.7 (C-4'), 65.4 (C-6’), 64.7 (C-5), $55.6\left(\mathrm{OCH}_{3}\right), 41.6(\mathrm{C}-2)$. $\quad$ HRMS (ESI) calcd $(\mathrm{M}+\mathrm{Na})^{+} \mathrm{C}_{40} \mathrm{H}_{38} \mathrm{O}_{13}: 749.2205$. Found: 749.2208. 


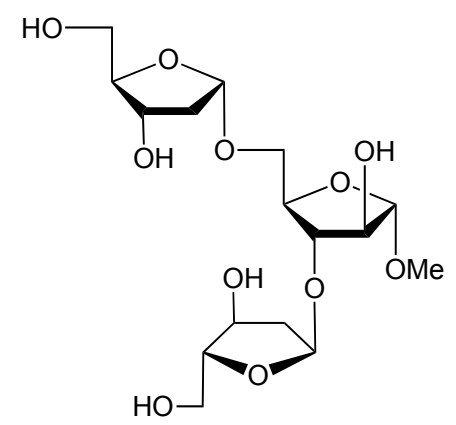

Methyl 5-O-(2-deoxy- $\alpha$-D-erythro-pentofuranosyl)-3-O-(2-deoxy- $\alpha$-D-erythro-pentofuranosyl)- $\alpha$-D-arabinofuranoside (45)

To a solution of $\mathbf{5 1}(49 \mathrm{mg}, 49 \mu \mathrm{mol})$ in anhydrous ethanol $(5 \mathrm{~mL})$ was added a catalytic amount of Raney Nickel (W4). The reaction mixture was stirred vigorously under a flow of hydrogen at room temperature until the TLC showed no starting material. The catalyst was filtered and the filtrate was concentrated. The resulting residue was then dissolved in $5 \mathrm{~mL}$ $\left(\mathrm{CH}_{2} \mathrm{Cl}_{2}-\mathrm{CH}_{3} \mathrm{OH}, 1: 1\right)$ and the $\mathrm{pH}$ of the solution was adjusted to $\sim 11$ by adding $1 \mathrm{M}$ $\mathrm{NaOCH}_{3}$ in $\mathrm{CH}_{3} \mathrm{OH}$. The reaction mixture was stirred at room temperature for $8 \mathrm{~h}$. After neutralization with IR-120 $\mathrm{H}^{+}$resin, the reaction mixture was filtered and the fitrate was concentrated. The residue was purified by chromatography on Iatrobeads in $6: 1$ $\mathrm{CH}_{2} \mathrm{Cl}_{2}-\mathrm{CH}_{3} \mathrm{OH}$ to obtain 45 in $63 \%$ yield $(12.2 \mathrm{mg})$ for two steps as a white foam: $R_{f} 0.22$ $\left(5: 1 \mathrm{CH}_{2} \mathrm{Cl}_{2}-\mathrm{CH}_{3} \mathrm{OH}\right) ;[\alpha]_{\mathrm{D}}+153.4\left(c 0.1, \mathrm{CH}_{2} \mathrm{Cl}_{2}\right) ;{ }^{1} \mathrm{H} \mathrm{NMR}\left(500 \mathrm{MHz}, \mathrm{CD}_{3} \mathrm{OD}, \delta_{\mathrm{H}}\right) 5.30$ (dd, 1H, $\left.J=5.5,1.7 \mathrm{~Hz}, \mathrm{H}-1^{\prime}\right), 5.21$ (dd, 1H, $J=5.3,1.4 \mathrm{~Hz}, \mathrm{H}-1^{\prime}$ ), 4.75 (s, 1H, H-1), 4.15-4.07 (m, 3H, H-4, H-3', H-3”), 4.05-3.99 (m, 3H, H-4' or H-4”, H-2, H-3), 3.95-3.88 (m, 2H, H-4" or H-4', H-5a), 3.72-3.62 (m, 3H, H-5b, H-5a', H-5a”), 3.59-3.53 (m, 2H, H-5b', H-5b"), 3.35 (s, 3H, $\mathrm{OCH}_{3}$ ), 2.37-2.24 (m, 2H, H-2a', H-2a”), 1.94-1.85 (m, 2H, H-2b', H-2b"); ${ }^{13} \mathrm{C}$ NMR (125 MHz, $\left.\mathrm{CDCl}_{3}, \delta_{\mathrm{C}}\right) 110.8$ (C-1), 105.3 (C-1"), 105.0 (C-1'), 87.2 (C-4' or C-4”), 86.9 (C-4" or C-4'), 84.4 (C-2 or C-3), 82.9 (C-4), 82.0 (C-3 or C-2), 
72.7 (C-3' or C-3”), 72.4 (C-3”' or C-3'), 67.7 (C-5), 63.3 (C-5' or C-5”), 63.1 (C-5” or C-5'), 55.2 (OㅡH3), 42.4 (C-2' or C-2”), 42.3 (C-2” or C-2'). HRMS (ESI) calcd (M+Na) ${ }^{+}$ $\mathrm{C}_{16} \mathrm{H}_{28} \mathrm{O}_{11} \mathrm{Na}: 419.1524$. Found: 419.1521.

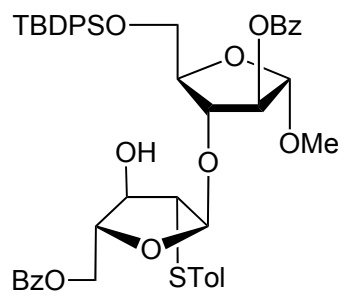

Methyl 5-O-t-butyldiphenylsilyl-3-O-(5-O-benzoyl-2-deoxy-2-p-thiotolyl- $\alpha$-D-arabinofuranosyl)-2-O-benzoyl- $\alpha$-D-arabinofuranoside (47)

To a mixture of donor 2 (72 $\mathrm{mg}, 0.21 \mathrm{mmol})$, alcohol $46^{7}(531 \mathrm{mg}, 1.05 \mathrm{mmol})$, and $4 \AA$ molecular sieves were added $\mathrm{CH}_{2} \mathrm{Cl}_{2}(10 \mathrm{~mL})$. The mixture was stirred for $20 \mathrm{~min}$ at $0{ }^{\circ} \mathrm{C}$. Copper triflate (15 mg, $0.04 \mathrm{mmol})$ was added and the reaction was stirred until TLC showed that no more donor was left. After neutralization by triethylamine, the solution was concentrated to yield a crude residue that was purified by chromatography $4: 1$ hexane-EtOAc to obtain 47 (152 mg, 85\%) as a colorless oil: $R_{f} 0.57\left(2: 1\right.$ hexane-EtOAc); $[\alpha]_{\mathrm{D}}+21.1(c 1.5$, $\mathrm{CH}_{2} \mathrm{Cl}_{2}$ ); ${ }^{1} \mathrm{H}$ NMR (500 MHz, $\left.\mathrm{CDCl}_{3}, \delta_{\mathrm{H}}\right)$ 8.02-7.95 (m, 4H, Ar), 7.71-7.67 (m, 4H, Ar), 7.59-7.52 (m, 2H, Ar), 7.43-7.30 (m, $12 \mathrm{H}, \mathrm{Ar}), 7.09-7.05(\mathrm{~m}, 2 \mathrm{H}, \mathrm{Ar}), 5.37\left(\mathrm{~d}, 1 \mathrm{H}, J_{1,2}=\right.$ $1.6 \mathrm{~Hz}, \mathrm{H}-1), 5.20$ (d, 1H, $\left.J_{2}^{\prime}, 3^{\prime}=1.5 \mathrm{~Hz}, \mathrm{H}-2^{\prime}\right), 5.06$ (s, 1H, H-1'), 4.48-4.38 (m, 3H, H-3', H-5a, H-5b), 4.28-4.20 (m, 1H, H-4'), 4.18-4.14 (m, 1H, H-4), 4.09-4.03 (m, 1H, H-3), 3.96 $\left(\mathrm{dd}, 1 \mathrm{H}, J_{5 \mathrm{a}^{\prime}, 5 \mathrm{~b}}{ }^{\prime}=11.2 \mathrm{~Hz}, J_{5 \mathrm{a}^{\prime}, 4^{\prime}}=4.8 \mathrm{~Hz}, \mathrm{H}-5 \mathrm{a}^{\prime}\right), 3.90\left(\mathrm{dd}, 1 \mathrm{H}, J_{5 \mathrm{~b}^{\prime}, 5 \mathrm{a}^{\prime}}=11.2 \mathrm{~Hz}, J_{5 \mathrm{~b}^{\prime}, 4^{\prime}}=3.9\right.$ Hz, H-5b'), 3.67 (dd, 1H, $\left.J_{2,3}=3.8 \mathrm{~Hz}, J_{2,1}=1.6 \mathrm{~Hz}, \mathrm{H}-2\right), 3.42\left(\mathrm{~s}, 3 \mathrm{H}, \mathrm{OCH}_{3}\right), 2.62(\mathrm{~d}, 1 \mathrm{H}$, $\left.J_{\mathrm{OH}, 3}=8.5 \mathrm{~Hz}, \mathrm{OH}\right), 2.27\left(\mathrm{~s}, 3 \mathrm{H}\right.$, tolyl $\left.\mathrm{CH}_{3}\right), 1.03\left(\mathrm{~s}, 9 \mathrm{H}, \mathrm{C}\left(\mathrm{CH}_{3}\right)_{3}\right) ;{ }^{13} \mathrm{C} \mathrm{NMR}(125 \mathrm{MHz}$, 
$\left.\mathrm{CDCl}_{3}, \delta_{\mathrm{C}}\right) 166.4(\underline{\mathrm{C}}=\mathrm{O}), 165.4(\underline{\mathrm{C}}=\mathrm{O}), 137.6(\mathrm{Ar}), 135.62(\mathrm{Ar} \times 2), 135.59(\mathrm{Ar} \times 2), 133.34$ (Ar), $133.32(\mathrm{Ar}), 133.2(\mathrm{Ar}), 133.1(\mathrm{Ar}), 131.6(\mathrm{Ar} \times 2), 130.0(\mathrm{Ar} \times 2), 129.85(\mathrm{Ar} \times 2)$, $129.79(\mathrm{Ar} \times 2), 129.73(\mathrm{Ar}), 129.67(\mathrm{Ar}), 129.6(\mathrm{Ar}), 129.3(\mathrm{Ar}), 128.5(\mathrm{Ar}), 128.4(\mathrm{Ar} \times 2)$, $128.3(\mathrm{Ar} \times 2), 127.72(\mathrm{Ar} \times 2), 127.68(\mathrm{Ar} \times 2), 107.3(\mathrm{C}-1), 106.9\left(\mathrm{C}-1^{\prime}\right), 83.5\left(\mathrm{C}-4^{\prime}\right), 83.0$ (C-4), 82.8 (C-2'), 81.2 (C-3'), 77.0 (C-3), 64.0 (C-5), 63.1 (C-5'), 58.3 (C-2), 54.8 ( OㅁH $\left._{3}\right)$, $26.79\left(\mathrm{C}\left(\underline{\mathrm{CH}}_{3}\right)_{3}\right), 26.76\left(\mathrm{C}\left(\underline{\mathrm{CH}}_{3}\right)_{3}\right), 21.0\left(\right.$ tolyl $\left.\underline{\mathrm{C}} \mathrm{H}_{3}\right), 19.3\left(\mathrm{Si} \underline{\mathrm{C}}\left(\mathrm{CH}_{3}\right)_{3}\right)$. HRMS (ESI) calcd $(\mathrm{M}+\mathrm{Na})^{+} \mathrm{C}_{48} \mathrm{H}_{52} \mathrm{O}_{10} \mathrm{SiSNa}$ : 871.2943. Found: 871.2941.

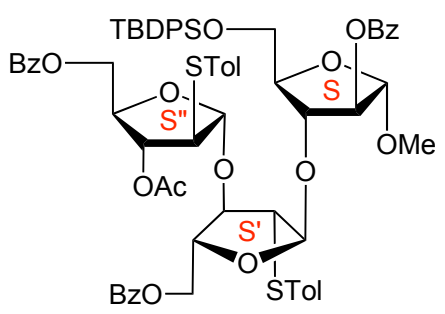

Methyl 5-O-t-butyldiphenylsilyl-3-O-(5-O-benzoyl-3-O-((5-O-benzoyl-3-O -acetyl2-deoxy-2-p-thiotolyl- $\alpha$-D-arabinofuranosyl))-2-deoxy-2-p-thiotolyl- $\alpha$-D-arabinofuranosyl)-2-O-benzoyl- $\alpha$-D-arabinofuranoside (48-Acetate)

To a mixture of donor 2 (209 mg, $0.61 \mathrm{mmol})$, alcohol $\mathbf{4 6}^{7}$ (309 mg, $\left.0.61 \mathrm{mmol}\right)$, and $4 \AA$ molecular sieves was added $\mathrm{CH}_{2} \mathrm{Cl}_{2}(10 \mathrm{~mL})$. The mixture was stirred for $20 \mathrm{~min}$ at room temperature. Copper triflate $(221 \mathrm{mg}, 0.61 \mathrm{mmol})$ was added and the reaction was stirred until TLC showed that no more donor was left. After neutralization by triethylamine, the solution was concentrated to yield a crude residue that was purified by chromatography $4: 1$, hexane-EtOAc to obtain a mixture of the desired disaccharide $\mathbf{4 7}$ as well as trisaccharide $\mathbf{4 8}$ (342 $\mathrm{mg})$ in a $4: 1$ ratio in $66 \%$ yield. Separation required acetylation $\left(\mathrm{Ac}_{2} \mathrm{O}\right.$, pyridine) of the mixture followed by chromatography in 4:1 hexane-EtOAc, which provided 48-Acetate 
as a colorless oil: $R_{f} 0.67\left(2: 1\right.$ hexane-EtOAc); $[\alpha]_{\mathrm{D}}+16.6\left(c \quad 0.2, \mathrm{CH}_{2} \mathrm{Cl}_{2}\right){ }^{1} \mathrm{H}$ NMR (400 $\left.\mathrm{MHz}, \mathrm{CDCl}_{3}, \delta_{\mathrm{H}}\right)$ 8.08-8.04 (m, 2H, Ar), 7.99-7.91 (m, 4H, Ar), 7.70-7.45 (m, 4H, Ar), 7.58-7.45 (m, 3H, Ar), 7.42-7.28 (m, 14H, Ar), 7.24-7.20 (m, 2H, Ar), 7.09 (d, 2H, $J=8.0$ $\mathrm{Hz}, \mathrm{Ar}), 6.99(\mathrm{~d}, 2 \mathrm{H}, J=8.0 \mathrm{~Hz}, \mathrm{Ar}), 5.26\left(\mathrm{~d}, 1 \mathrm{H}, J_{1^{\prime}, 2^{\prime}}=1.8 \mathrm{~Hz}, \mathrm{H}-1^{\prime}\right), 5.18\left(\mathrm{~d}, 1 \mathrm{H}, J_{2,1}=1.6\right.$

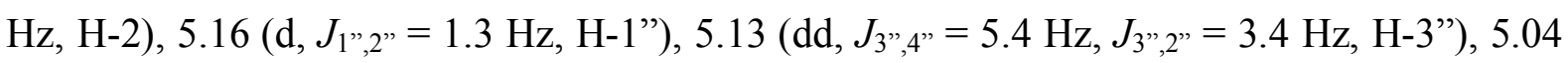
(br s, H-1), $4.51\left(\mathrm{dd}, 1 \mathrm{H}, J_{5 \mathrm{a}^{\prime}, 5 \mathrm{~b}}{ }^{\prime}=12.0 \mathrm{~Hz}, J_{5 \mathrm{a}^{\prime}, 4^{\prime}}=3.5 \mathrm{~Hz}, \mathrm{H}-5 \mathrm{a} \mathrm{a}^{\prime \prime}\right), 4.46\left(\mathrm{dd}, 1 \mathrm{H}, J_{5 \mathrm{a}^{\prime}, 5 \mathrm{~b}^{\prime}}=\right.$ 12.1 Hz, $\left.J_{5 \mathrm{a}^{\prime}, 4^{\prime}}=3.0 \mathrm{~Hz}, \mathrm{H}-5 \mathrm{a}^{\prime}\right), 4.43-4.37$ (m, 2H, H-3, H-5b”), 4.34-4.29 (m, 2H, H-4”, H-5b'), 4.23-4.16 (m, 2H, H-4, H-4'), 4.09 (dd, 1H, $J_{3^{\prime}, 4^{\prime}}=7.0 \mathrm{~Hz}, J_{3^{\prime}, 2^{\prime}}=4.7 \mathrm{~Hz}, \mathrm{H}-3^{\prime}$ '), 3.94 $\left(\mathrm{dd}, 1 \mathrm{H}, J_{5 \mathrm{a}, 5 \mathrm{~b}}=11.4 \mathrm{~Hz}, J_{5 \mathrm{a}, 4}=4.6 \mathrm{~Hz}, \mathrm{H}-5 \mathrm{a}\right), 3.88\left(\mathrm{dd}, 1 \mathrm{H}, J_{5 \mathrm{a}, 5 \mathrm{~b}}=11.4 \mathrm{~Hz}, J_{5 \mathrm{a}, 4}=3.2 \mathrm{~Hz}\right.$, $\mathrm{H}-5 \mathrm{~b}), 3.70\left(\mathrm{dd}, 1 \mathrm{H}, J_{2 ",}{ }^{\prime \prime}=3.4 \mathrm{~Hz}, J_{2 ",}{ }^{\prime \prime}=1.3 \mathrm{~Hz}, \mathrm{H}-2^{\prime \prime}\right), 3.56\left(\mathrm{dd}, 1 \mathrm{H}, J_{2^{\prime}, 3^{\prime}}=4.7 \mathrm{~Hz}, J_{2}{ }^{\prime}, 1^{\prime}=\right.$ $1.8 \mathrm{~Hz}, \mathrm{H}-2$ '), $3.45\left(\mathrm{~s}, 3 \mathrm{H}, \mathrm{OCH}_{3}\right), 2.29\left(\mathrm{~s}, 3 \mathrm{H}\right.$, tolyl $\left.\mathrm{CH}_{3}\right), 2.22\left(\mathrm{~s}, 3 \mathrm{H}\right.$, tolyl $\left.\mathrm{CH}_{3}\right), 2.02(\mathrm{~s}$, $3 \mathrm{H}$, acetyl $\left.\mathrm{CH}_{3}\right), 1.01\left(\mathrm{~s}, 9 \mathrm{H}, \mathrm{C}\left(\mathrm{CH}_{3}\right)_{3}\right) ;{ }^{13} \mathrm{C} \mathrm{NMR}\left(125 \mathrm{MHz}, \mathrm{CDCl}_{3}, \delta_{\mathrm{C}}\right) 170.0(\underline{\mathrm{C}}=\mathrm{O}), 166.1$ $(\underline{\mathrm{C}}=\mathrm{O}), 166.1(\underline{\mathrm{C}}=\mathrm{O}), 165.5(\underline{\mathrm{C}}=\mathrm{O}), 137.8(\mathrm{Ar}), 137.5(\mathrm{Ar}), 135.64(\mathrm{Ar} \times 2), 135.59(\mathrm{Ar} \times 2)$, 133.34 (Ar), $133.30(\mathrm{Ar}), 133.28(\mathrm{Ar}), 133.01(\mathrm{Ar}), 133.00(\mathrm{Ar}), 131.7(\mathrm{Ar} \times 2), 131.5(\mathrm{Ar} \times$ 2), $130.1(\mathrm{Ar} \times 2), 130.0(\mathrm{Ar} \times 2), 129.92(\mathrm{Ar}), 129.85(\mathrm{Ar} \times 4), 129.75(\mathrm{Ar}), 129.71(\mathrm{Ar} \times 2)$, $129.69(\mathrm{Ar}), 129.6(\mathrm{Ar} \times 2), 129.5(\mathrm{Ar}), 129.4(\mathrm{Ar}), 128.4(\mathrm{Ar} \times 2), 128.32(\mathrm{Ar} \times 2), 128.29$ $(\mathrm{Ar} \times 2), 127.7(\mathrm{Ar} \times 2), 127.6(\mathrm{Ar} \times 2), 107.9\left(\mathrm{C}-1^{\prime}\right), 107.2\left(\mathrm{C}-1^{\prime \prime}\right), 107.0(\mathrm{C}-1), 83.4(\mathrm{C}-4$ or C-4'), 83.2 (C-2), 81.0 (C-3' and C-4'’), 80.7 (C-3'), 79.8 (C-4' or C-4), 77.3 (C-3), 63.8 (C-5”), 62.95 (C-5'), 62.9 (C-5), 56.6 (C-2'), 56.2 (C-2”), $54.8\left(\mathrm{OC}_{3}{ }_{3}\right), 26.7\left(\mathrm{C}^{\prime}\left(\underline{\mathrm{CH}}_{3}\right)_{3}\right), 21.1$ (tolyl $\left.\underline{\mathrm{CH}}_{3}\right), 21.0$ (tolyl $\left.\underline{\mathrm{CH}}_{3}\right), 20.8\left(\right.$ acetyl $\left.\underline{\mathrm{CH}}_{3}\right), 19.3\left(\mathrm{SiC}\left(\mathrm{CH}_{3}\right)_{3}\right)$. HRMS (ESI) calcd $(\mathrm{M}+\mathrm{Na})^{+} \mathrm{C}_{69} \mathrm{H}_{72} \mathrm{O}_{15} \mathrm{SiS}_{2} \mathrm{Na}: 1255.3974$. Found: 1255.3973. 


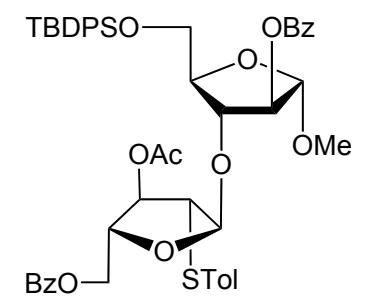

\section{Methyl 5-O-t-butyldiphenylsilyl-3-O-(5-O-benzoyl-3-O-acetyl-2-deoxy-2-p-thiotolyl- $\alpha$ -} D-arabinofuranosyl)-2- $O$-benzoyl- $\alpha$-D-arabinofuranoside (49)

To a mixture of compound $47(236 \mathrm{mg}, 0.28 \mathrm{mmol})$ and pyridine $(1 \mathrm{~mL})$ was added acetic anhydride (32 $\mu \mathrm{L}, 0.34 \mathrm{mmol})$ and DMAP $(2 \mathrm{mg}, 14 \mu \mathrm{mol})$. The reaction was stirred for $4 \mathrm{~h}$ at room temperature. After addition of $\mathrm{CH}_{3} \mathrm{OH}$ to quench the reaction, the mixture was concentrated and the residue purified by chromatography 5:1 hexane-EtOAc to obtain the 49 (247 mg, quant) as a colorless oil: $R_{f} 0.70\left(2: 1\right.$ hexane-EtOAc); $[\alpha]_{\mathrm{D}}+14.5\left(c 0.1, \mathrm{CH}_{2} \mathrm{Cl}_{2}\right)$ ${ }^{1} \mathrm{H}$ NMR $\left(500 \mathrm{MHz}, \mathrm{CDCl}_{3}, \delta_{\mathrm{H}}\right)$ 8.06-7.96 (m, 4H, Ar), 7.72-7.66 (m, 4H, Ar), 7.58-7.50 (m, 2H, Ar), 7.42-7.31 (m, 12H, Ar), 7.08-7.05 (m, 2H, Ar), 5.35 (d, 1H, $\left.J_{1,2}=1.4 \mathrm{~Hz}, \mathrm{H}-1\right)$, $5.20\left(\mathrm{~d}, 1 \mathrm{H}, J_{2}{ }^{\prime}, 3^{\prime}=1.8 \mathrm{~Hz}, \mathrm{H}-2^{\prime}\right), 5.16\left(\mathrm{dd}, 1 \mathrm{H}, J_{3,4}=5.4 \mathrm{~Hz}, J_{3,2}=3.5 \mathrm{~Hz}, \mathrm{H}-3\right), 5.04(\mathrm{~s}, 1 \mathrm{H}$, H-1'), 4.47-4.43 (m, 2H, H-3', H-5a), 4.37 (dd, 1H, $\left.J_{5 \mathrm{~b}, 5 \mathrm{a}}=12.0 \mathrm{~Hz}, J_{5 \mathrm{~b}, 4}=5.0 \mathrm{~Hz}, \mathrm{H}-5 \mathrm{~b}\right)$, 4.22-4.17 (m, 2H, H-4, H-4'), 3.96-3.87 (m, 2H, H-5a', H-5b'), 3.75 (dd, 1H, $J_{2,3}=3.5$ Hz, $\left.J_{2,1}=1.4 \mathrm{~Hz}, \mathrm{H}-2\right), 3.45\left(\mathrm{~s}, 3 \mathrm{H}, \mathrm{OCH}_{3}\right), 2.26\left(\mathrm{~s}, 3 \mathrm{H}\right.$, tolyl $\left.\mathrm{CH}_{3}\right), 2.02\left(\mathrm{~s}, 3 \mathrm{H}\right.$, acetyl $\left.\mathrm{CH}_{3}\right), 1.02$ (s, 9H, C(CH3) $\left.)_{3}\right) ;{ }^{13} \mathrm{C}$ NMR $\left(500 \mathrm{MHz}, \mathrm{CDCl}_{3}, \delta_{\mathrm{C}}\right) 170.0(\mathrm{C}=\mathrm{O}), 166.1(\mathrm{C}=\mathrm{O}), 165.5(\mathrm{C}=\mathrm{O})$, $137.8(\mathrm{Ar}), 135.64(\mathrm{Ar} \times 2), 135.57(\mathrm{Ar} \times 2), 133.3(\mathrm{Ar}), 133.2(\mathrm{Ar}), 133.0(\mathrm{Ar}), 131.9(\mathrm{Ar} \times$ 2), $130.0(\mathrm{Ar} \times 2), 129.84(\mathrm{Ar} \times 2), 129.82(\mathrm{Ar} \times 2), 129.7(\mathrm{Ar}), 129.6(\mathrm{Ar}), 129.5(\mathrm{Ar}), 129.3$ $(\mathrm{Ar}), 128.4(\mathrm{Ar} \times 2), 128.33(\mathrm{Ar} \times 2), 128.26(\mathrm{Ar} \times 2), 127.7(\mathrm{Ar} \times 2), 127.6(\mathrm{Ar} \times 2), 107.2$ (C-1), 107.0 (C-1'), 83.2 (C-4' or C-4), 83.0 (C-2'), 80.7 (C-3'), 80.6 (C-4 or C-4'), 77.4 (C-3), 63.7 (C-5), 62.9 (C-5'), $56.3(\mathrm{C}-2), 54.8\left(\mathrm{O}^{\mathrm{C}} \mathrm{H}_{3}\right), 26.8\left(\mathrm{SiC}\left(\underline{\mathrm{CH}}_{3}\right)_{3}\right), 21.0$ (tolyl $\left.\underline{\mathrm{CH}}_{3}\right)$, 
20.8 (acetyl $\left.\underline{\mathrm{CH}}_{3}\right), 19.3\left(\mathrm{SiC}\left(\mathrm{CH}_{3}\right)_{3}\right) . \quad$ HRMS (ESI) calcd $(\mathrm{M}+\mathrm{Na})^{+} \mathrm{C}_{50} \mathrm{H}_{54} \mathrm{O}_{11} \mathrm{SiSNa}$ : 913.3048, Found: 913.3048 .

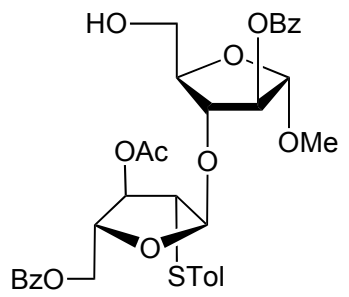

Methyl 3-O-(5-O-benzoyl-3-O-acetyl-2-deoxy-2-p-thiotolyl- $\alpha$-D-arabinofuranosyl)-2- $O$ benzoyl- $\alpha$-D-arabinofuranoside (50)

To a solution of compound $49(247 \mathrm{mg}, 0.28 \mathrm{mmol})$ in THF $(10 \mathrm{~mL})$ at room temperature, 1M TBAF in THF (0.44 mL, $0.44 \mathrm{mmol})$ was added. The reaction mixture was stirred for 5 $\mathrm{h}$ and concentrated to give an oily residue that was purified by chromatography $2: 1$ hexane-EtOAc to obtain 56 in 79\% (143 mg) for two steps as a colorless oil: $R_{f} 0.25(2: 1$ hexane-EtOAc); $[\alpha]_{\mathrm{D}}+25.2\left(c 0.1, \mathrm{CH}_{2} \mathrm{Cl}_{2}\right) ;{ }^{1} \mathrm{H}$ NMR $\left(400 \mathrm{MHz}, \mathrm{CDCl}_{3}, \delta_{\mathrm{H}}\right)$ 8.10-8.07 (m, 2H, Ar), 8.02-7.98 (m, 2H, Ar), 7.61-7.54 (m, 2H, Ar), 7.47-7.41 (m, 4H, Ar), 7.33 (d, 2H, $J$ $=8.1 \mathrm{~Hz}, \mathrm{Ar}), 7.05(\mathrm{~d}, 2 \mathrm{H}, J=7.9 \mathrm{~Hz}, \mathrm{Ar}), 5.34\left(\mathrm{~d}, 1 \mathrm{H}, J_{1,2}=1.7 \mathrm{~Hz}, \mathrm{H}-1\right), 5.18\left(\mathrm{~d}, 1 \mathrm{H}, J_{2,3}=\right.$ $2.0 \mathrm{~Hz}, \mathrm{H}-2^{\prime}$ ), 5.15 (dd, 1H, $\left.J_{3,4}=5.6 \mathrm{~Hz}, J_{3,2}=3.9 \mathrm{~Hz}, \mathrm{H}-3\right), 5.01$ (s, 1H, H-1'), 4.60 (dd, 1H, $\left.J_{5 \mathrm{a}, 5 \mathrm{~b}}=12.0 \mathrm{~Hz}, J_{5 \mathrm{a}, 4}=3.5 \mathrm{~Hz}, \mathrm{H}-5 \mathrm{a}\right), 4.44\left(\mathrm{dd}, 1 \mathrm{H}, J_{5 \mathrm{~b}, 5 \mathrm{a}}=12.0 \mathrm{~Hz}, J_{5 \mathrm{~b}, 4}=6.0 \mathrm{~Hz}, \mathrm{H}-5 \mathrm{~b}\right)$, 4.38-4.33 (m, 1H, H-4), 4.31-4.27 (m, 1H, H-3'), 4.20-4.16 (m, 1H, H-4'), 3.93-3.78 (m, 2H, H-5a', H-5b'), 3.77 (dd, 1H, $\left.J_{2,3}=3.9 \mathrm{~Hz}, J_{2,1}=1.7 \mathrm{~Hz}, \mathrm{H}-2\right), 3.44\left(\mathrm{~s}, 3 \mathrm{H}, \mathrm{OCH}_{3}\right), 2.25$ (s, 3H, tolyl $\mathrm{CH}_{3}$ ), 2.05 (s, 3H, acetyl $\left.\mathrm{CH}_{3}\right), 1.98$ (br, $\left.1 \mathrm{H}, \mathrm{OH}\right) ;{ }^{13} \mathrm{C} \mathrm{NMR}\left(100 \mathrm{MHz}, \mathrm{CDCl}_{3}\right.$, $\left.\delta_{\mathrm{C}}\right) 169.9(\underline{\mathrm{C}}=\mathrm{O}), 166.1(\underline{\mathrm{C}}=\mathrm{O}), 165.3(\underline{\mathrm{C}}=\mathrm{O}), 137.9(\mathrm{Ar}), 133.4(\mathrm{Ar}), 133.0(\mathrm{Ar}), 132.1(\mathrm{Ar} \times$ 2), 129.9 $(\mathrm{Ar} \times 2), 129.73(\mathrm{Ar} \times 2), 129.67(\mathrm{Ar} \times 2), 129.6(\mathrm{Ar}), 129.1(\mathrm{Ar}), 129.0(\mathrm{Ar}), 128.4$ 
$(\mathrm{Ar} \times 2), 128.3(\mathrm{Ar} \times 2), 107.6(\mathrm{C}-1), 106.8\left(\mathrm{C}^{\prime} 1^{\prime}\right), 83.1\left(\mathrm{C}-2^{\prime}\right), 82.6\left(\mathrm{C}-4^{\prime}\right), 81.1\left(\mathrm{C}-3^{\prime}\right), 80.6$ (C-4), 77.2 (C-3), 63.7 (C-5), 61.6 (C-5'), 56.0 (C-2), $54.8\left(\mathrm{OCH}_{3}\right), 20.9$ (tolyl $\left.\underline{\mathrm{CH}}_{3}\right), 20.7$ (acetyl $\left.\underline{\mathrm{CH}}_{3}\right)$. HRMS (ESI) calcd $(\mathrm{M}+\mathrm{Na})^{+} \mathrm{C}_{34} \mathrm{H}_{36} \mathrm{O}_{11} \mathrm{SNa}$ : 675.1871. Found: 675.1872.

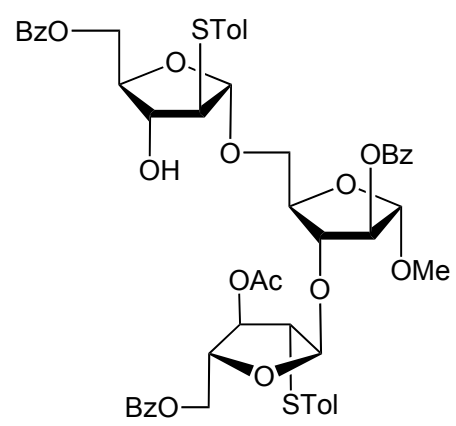

Methyl 5-O-(5-O-benzoyl-2-deoxy-2-p-thiotolyl- $\alpha$-D-arabinofuranosyl)-3-O-(5-O-benzoyl-3-O-acetyl-2-deoxy-2-p-thiotoly- $\alpha$-D-arabinofuranosyl)-2- $O$-benzoyl- $\alpha$-D-arabinofuranoside (51)

To a mixture of the donor 2 (14 mg, $0.04 \mathrm{mmol})$, alcohol $\mathbf{5 0}(131 \mathrm{mg}, 0.2 \mathrm{mmol})$, and $4 \AA$ molecular sieves was added $\mathrm{CH}_{2} \mathrm{Cl}_{2}(5 \mathrm{~mL})$. The mixture was stirred for 20 min at $0{ }^{\circ} \mathrm{C}$. Copper triflate $(3 \mathrm{mg}, 8.0 \mu \mathrm{mol})$ was added and the reaction mixture was stirred until TLC showed that no donor was left. After neutralization by triethylamine, the solution was concentrated to yield a crude residue that was purified by chromatography 5:1 hexane-EtOAc to obtain $51(30 \mathrm{mg}, 70 \%)$ as a colorless oil: $R_{f} 0.65\left(2: 1\right.$ hexane-EtOAc); $[\alpha]_{\mathrm{D}}+43.2(c 2.3$, $\mathrm{CH}_{2} \mathrm{Cl}_{2}$ ); ${ }^{1} \mathrm{H}$ NMR (500 MHz, $\left.\mathrm{CDCl}_{3}, \delta_{\mathrm{H}}\right)$ 8.07-8.04 (m, 4H, Ar), 8.01-7.98 (m, 2H, Ar), 7.58-7.50 (m, 3H, Ar), 7.46-7.38 (m, 6H, Ar), 7.34-7.30 (m, 2H, Ar), 7.28-7.24 (m, 2H, Ar), 7.06-7.03 (m, 4H, Ar), 5.34 (d, 1H, $J_{1^{\prime}, 2^{\prime}}=1.6 \mathrm{~Hz}, \mathrm{H}-1^{\prime}$ '), 5.17-5.14 (m, 3H, H-1', H-2, H-3'), $4.97(\mathrm{~s}, 1 \mathrm{H}, \mathrm{H}-1), 4.58\left(\mathrm{dd}, 1 \mathrm{H}, J_{5 \mathrm{a} ", 5 \mathrm{~b}}=12.0 \mathrm{~Hz}, J_{5 \mathrm{a} ", 4}=3.9 \mathrm{~Hz}, \mathrm{H}-5 \mathrm{a} "\right), 4.54-4.50(\mathrm{~m}, 2 \mathrm{H}$, H-5a', H-5b'), 4.45 (dd, 1H, $\left.J_{5 \mathrm{a}{ }^{\prime}, 5 \mathrm{~b} "}=12.0 \mathrm{~Hz}, J_{5 \mathrm{a} ", 4}=5.6 \mathrm{~Hz}, \mathrm{H}-5 \mathrm{~b} "\right), 4.38-4.32$ (m, 2H, 
H-4', H-4”), 4.24-4.19 (m, 2H, H-3, H-4), 4.08-4.04 (m, 1H, H-3”), 3.97 (dd, 1H, J5a,5b = $\left.10.7 \mathrm{~Hz}, J_{5 \mathrm{a}, 4}=4.9 \mathrm{~Hz}, \mathrm{H}-5 \mathrm{a}\right), 3.75-3.72\left(\mathrm{~m}, 2 \mathrm{H}, \mathrm{H}-2^{\prime}, \mathrm{H}-5 \mathrm{~b}\right), 3.62\left(\mathrm{dd}, 1 \mathrm{H}, J_{2 ",}{ }^{\prime \prime}=2.7 \mathrm{~Hz}\right.$, $J_{2}$, 1 " $\left.=1.1 \mathrm{~Hz}, \mathrm{H}-2 ”\right), 3.41\left(\mathrm{~s}, 3 \mathrm{H}, \mathrm{OCH}_{3}\right), 2.93(\mathrm{~d}, 1 \mathrm{H}, J=9.4 \mathrm{~Hz}, \mathrm{OH}), 2.27$ (s, 3H, tolyl $\left.\mathrm{CH}_{3}\right), 2.25\left(\mathrm{~s}, 3 \mathrm{H}\right.$, tolyl $\left.\mathrm{CH}_{3}\right), 2.02\left(\mathrm{~s}, 3 \mathrm{H}\right.$, acetyl $\left.\mathrm{CH}_{3}\right) ;{ }^{13} \mathrm{C} \mathrm{NMR}\left(100 \mathrm{MHz}, \mathrm{CDCl}_{3}, \delta_{\mathrm{C}}\right)$ $170.0(\underline{\mathrm{C}}=\mathrm{O}), 166.4(\underline{\mathrm{C}}=\mathrm{O}), 166.2(\underline{\mathrm{C}}=\mathrm{O}), 165.4(\underline{\mathrm{C}}=\mathrm{O}), 138.0(\operatorname{Ar}), 137.4(\mathrm{Ar}), 133.5$ (Ar), $133.14(\mathrm{Ar}), 133.11(\mathrm{Ar}) 132.1(\mathrm{Ar} \times 2), 131.3(\mathrm{Ar} \times 2), 130.0(\mathrm{Ar} \times 4), 129.9(\mathrm{Ar}), 129.8$ $(\mathrm{Ar} \times 2), 129.83(\mathrm{Ar} \times 2), 129.81(\mathrm{Ar} \times 2), 129.79(\mathrm{Ar} \times 2), 129.7(\mathrm{Ar}), 129.6(\mathrm{Ar}), 129.20$ (Ar), $129.17(\mathrm{Ar}), 128.5(\mathrm{Ar} \times 2), 128.4(\mathrm{Ar} \times 2), 107.4\left(\mathrm{C}-1^{\prime}\right), 107.1(\mathrm{C}-1$ '”), $106.9(\mathrm{C}-1)$, 83.9 (C-4' or C-4”), 83.0 (C-2 or C-3'), 81.5 (C-3 or C-4), 81.0 (C-4 or C-3), 80.6 (C-4” or C-4'), 77.4 (C-3' or C-2), 65.9 (C-5), 64.4 (C-5'), 63.9 (C-5”), 58.1 (C-2’), 56.2 (C-2'), 54.9 $\left(\mathrm{OCH}_{3}\right), 21.05$ (tolyl $\left.\underline{\mathrm{CH}}_{3}\right), 21.01$ (tolyl $\left.\underline{\mathrm{CH}}_{3}\right), 20.8$ (acetyl $\left.\underline{\mathrm{CH}}_{3}\right)$. HRMS (ESI) calcd $(\mathrm{M}+\mathrm{Na})^{+} \mathrm{C}_{53} \mathrm{H}_{54} \mathrm{O}_{15} \mathrm{~S}_{2} \mathrm{Na}: 1017.2796$. Found: 1017.2793. 


\section{References}

1. 14: Callam, C. S.; Gadikota, R. R.; Lowary, T. L. J. Org. Chem. 2001, 66, 4549-4558.

15: Ziegler, T.; Kovac, P.; Glaudemans, C. P. J. Carbohydr. Res. 1989, 194 185-198.

16: Zagar, C.; Scharf, H. D. Liebigs Ann. Chem. 1992, 7, 693-697.

17: Jiao, H.; Hindsgaul, O. Angew. Chem., Intl. Ed. 1999, 38, 346-348.

18: Yin, H.; D'Souza, F. W.; Lowary, T. L. J. Org. Chem. 2002, 67, 892-903.

2. (a) Patt, S.; Shoolery, J. N. J. Magn. Reson. 1982, 46, 535-539. (b) Torres, A. M.; Nakashima, T. T.; McClung, R. E. D. J. Magn. Reson. Ser. A 1993, 101, 285-289.

3. Gadikota, R.R.; Callam, C.S.; Lowary, T.L. Org. Lett. 2001, 3, 607-610.

4. Gadikota, R. R.; Callam, C. S.; Wagner, T.; Del Fraino, B.; Lowary, T. L. J. Am. Chem. Soc. 2003, 125, 4155-4165.

5. Zuurmond, H. M.; van der Klein, P. A. M.; van der Marel, G. A.; van Boom, J. H. Tetrahedron 1993, 49, 6501-6514.

6. Toshima K.; Mukaiyama S.; Nozaki Y.; Inokuchi H.; Nakata M.; Tatsuta K. J. Am. Chem. Soc. 1994, 116, 9042-9051

7. D’Souza, F. W.; Cheshev, P. E.; Ayers, J. D.; Lowary, T. L. J. Org. Chem. 1998, 63, 9037-9044. 


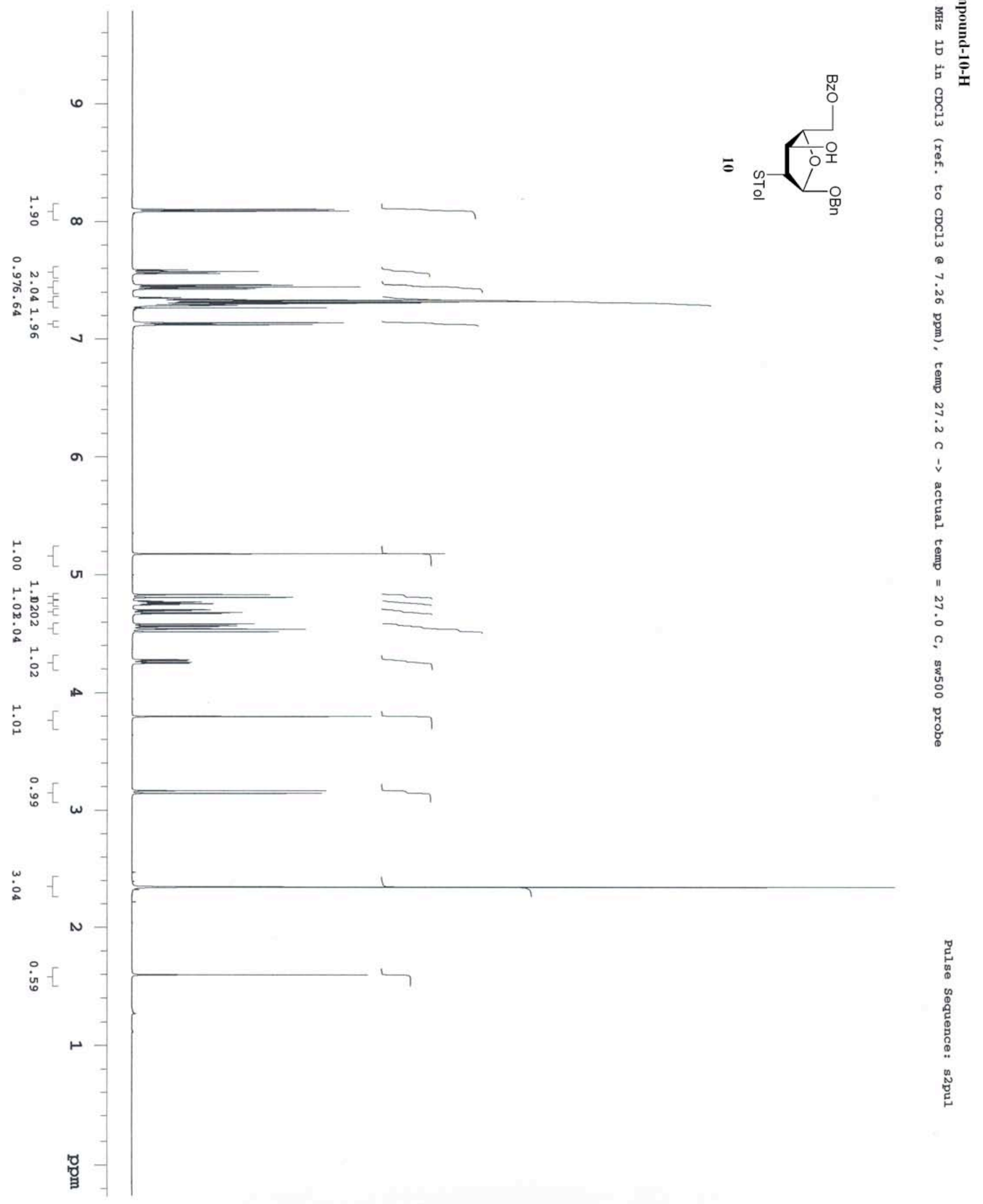



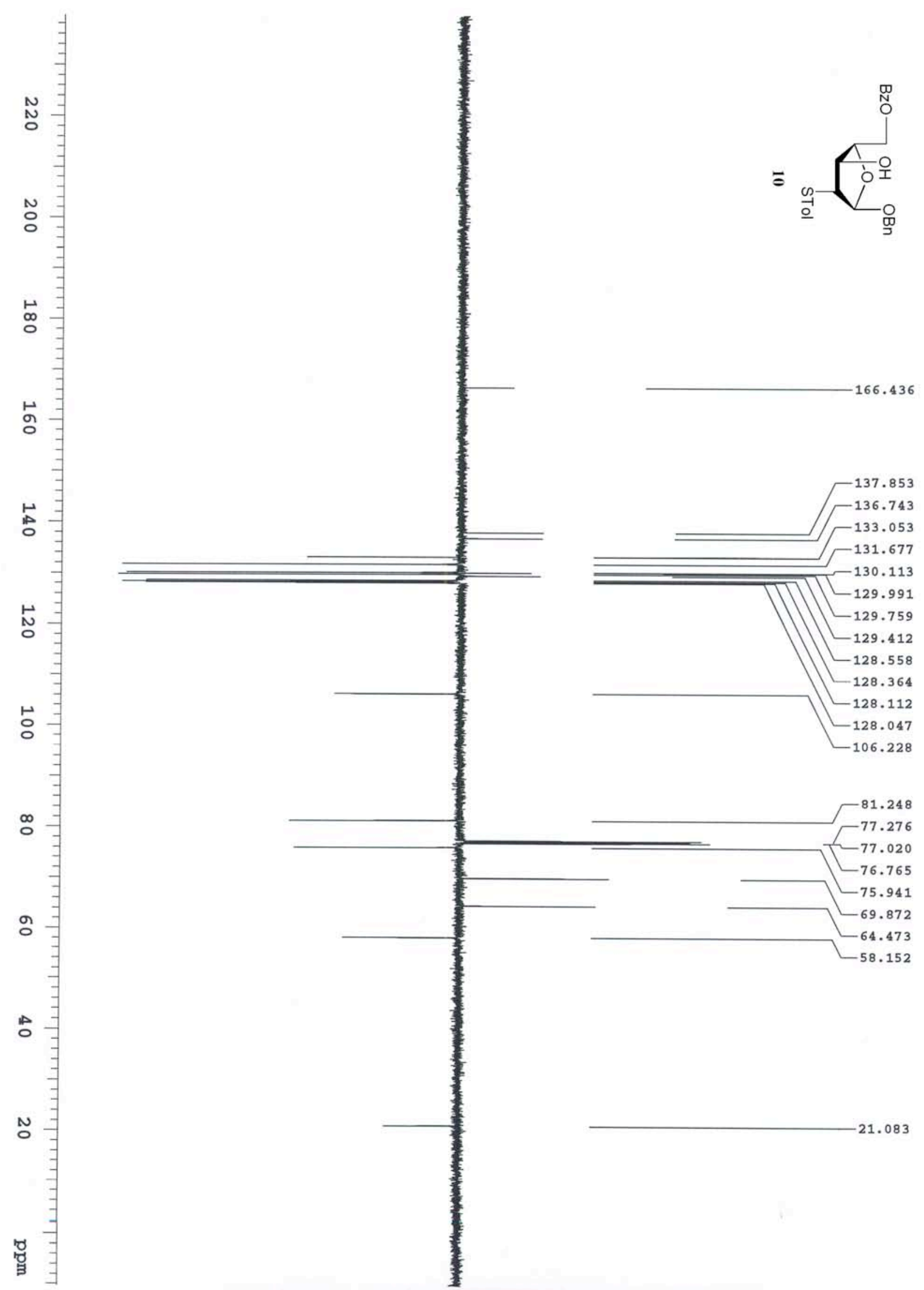

怘岕

惫宾

翼兽言

药

ํㅛㅇํํ

通

: ․ㅗㅇ

क

(5)

ำ

紫要

ㅇำ

".

兽苗

$-166.436$

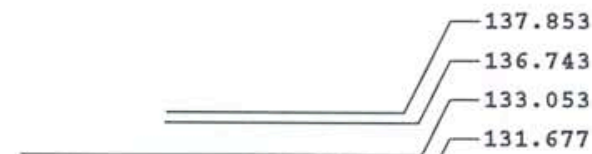

$-130.113$

129.991

$-129.412$

$-128.558$

$-128.364$

$-106.228$

$-81.248$

$-77.020$

$-76.765$

$-75.941$

$-69.872$

$-58.152$

21.083

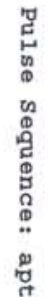




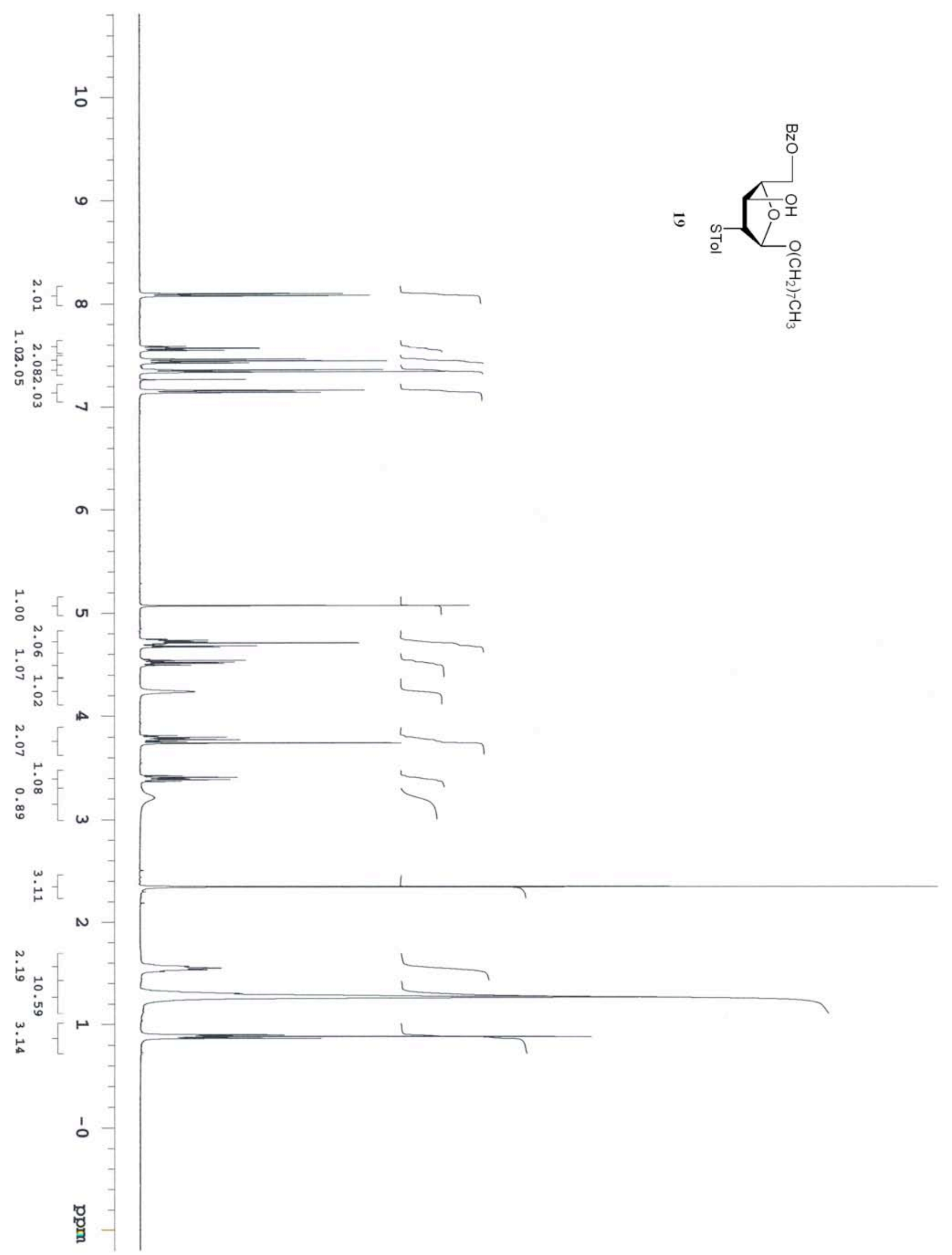

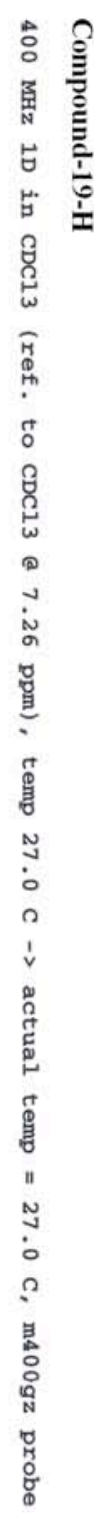




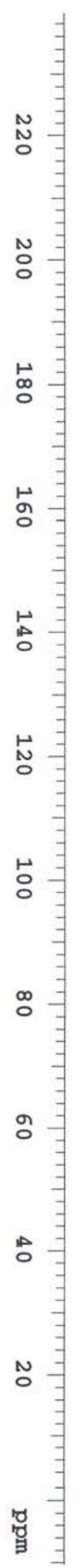
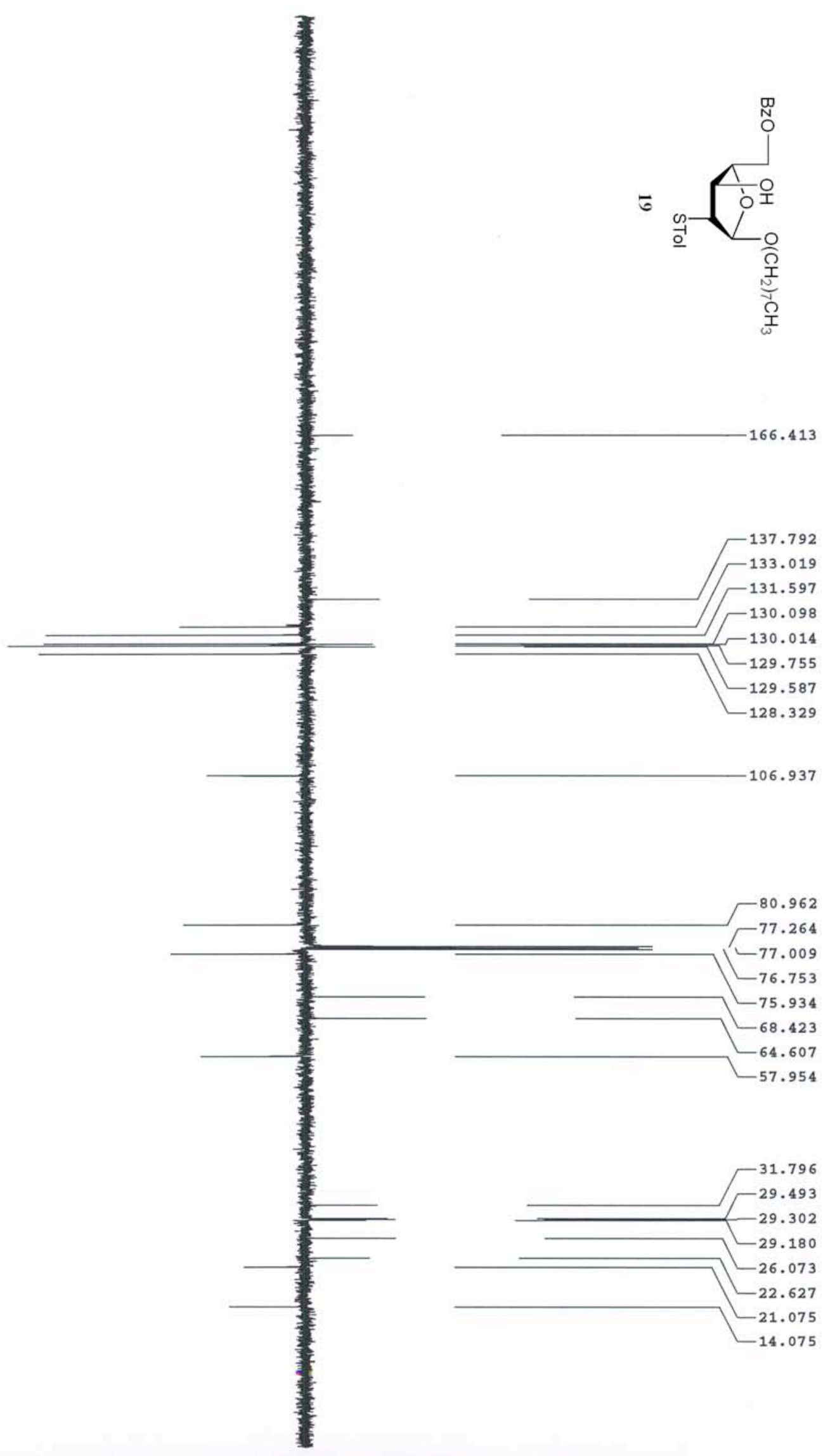

$-106.937$
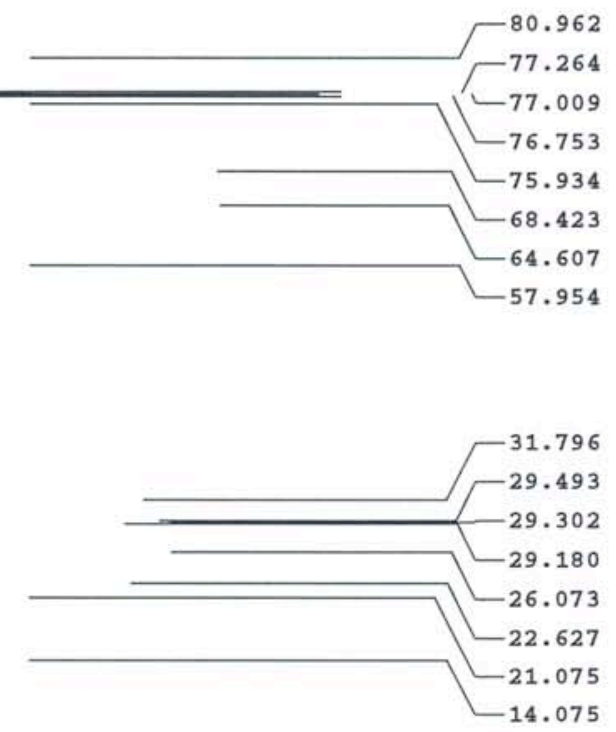

范
0
0
0
0
0
0
0
0
0
0
0
0
0
0 


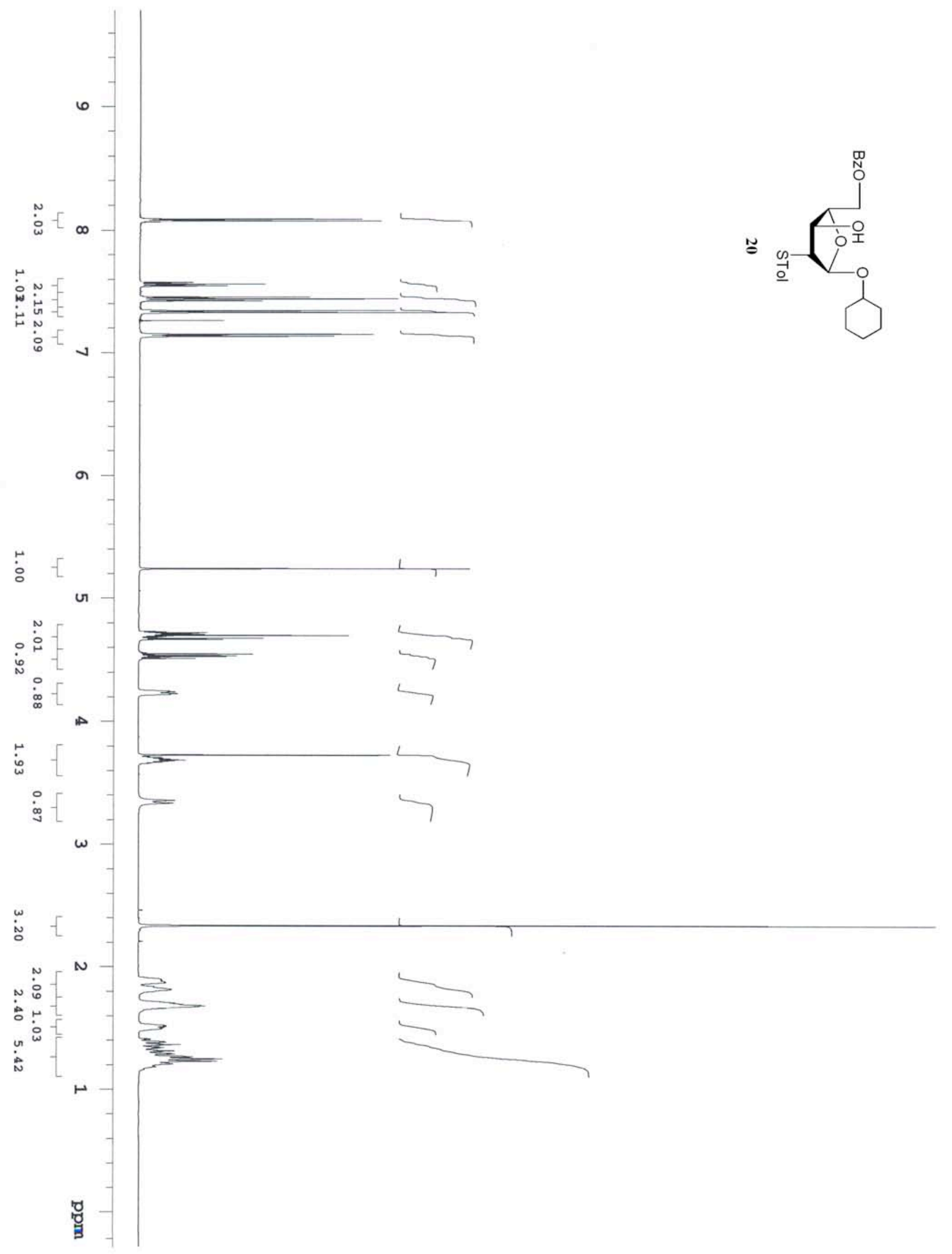




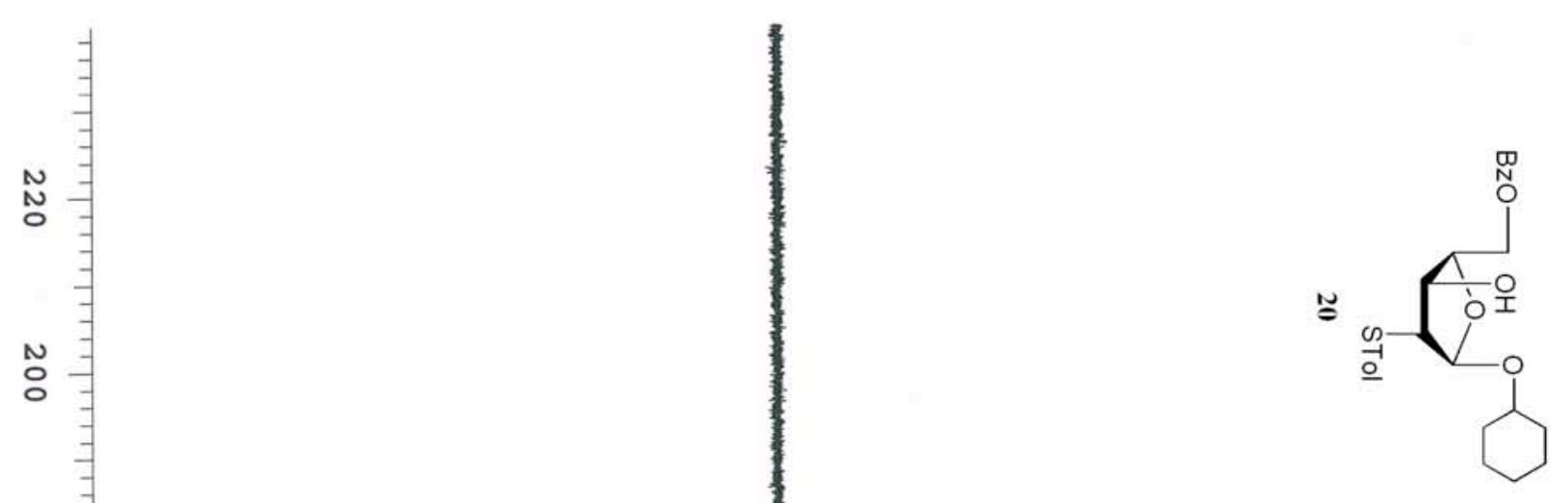

$\stackrel{+}{\infty}$

동

응

$-166.413$

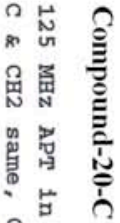

政

落

官

농

능

。

$\stackrel{\infty}{\circ}$

$\stackrel{\infty}{\circ}$

응

o -

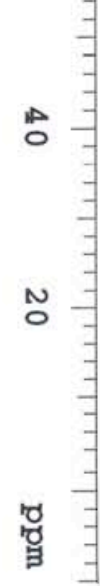
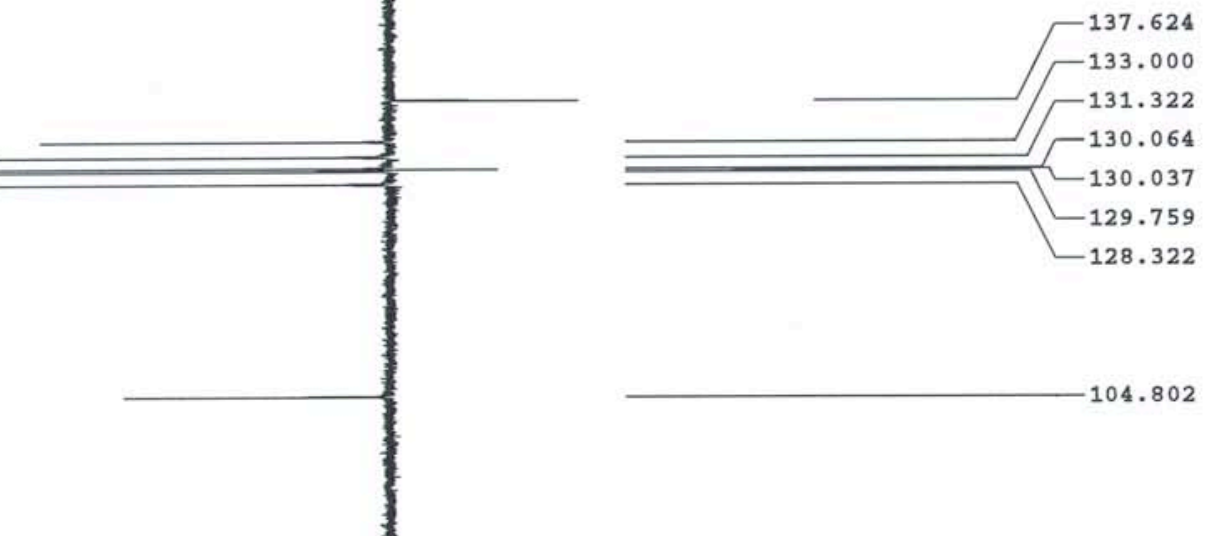

$\Gamma^{80.810}$ $-77.298$

$-76.788$

$-75.968$

$-75.758$

$-64.709$

$-58.011$

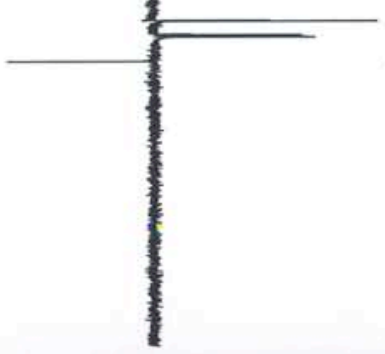




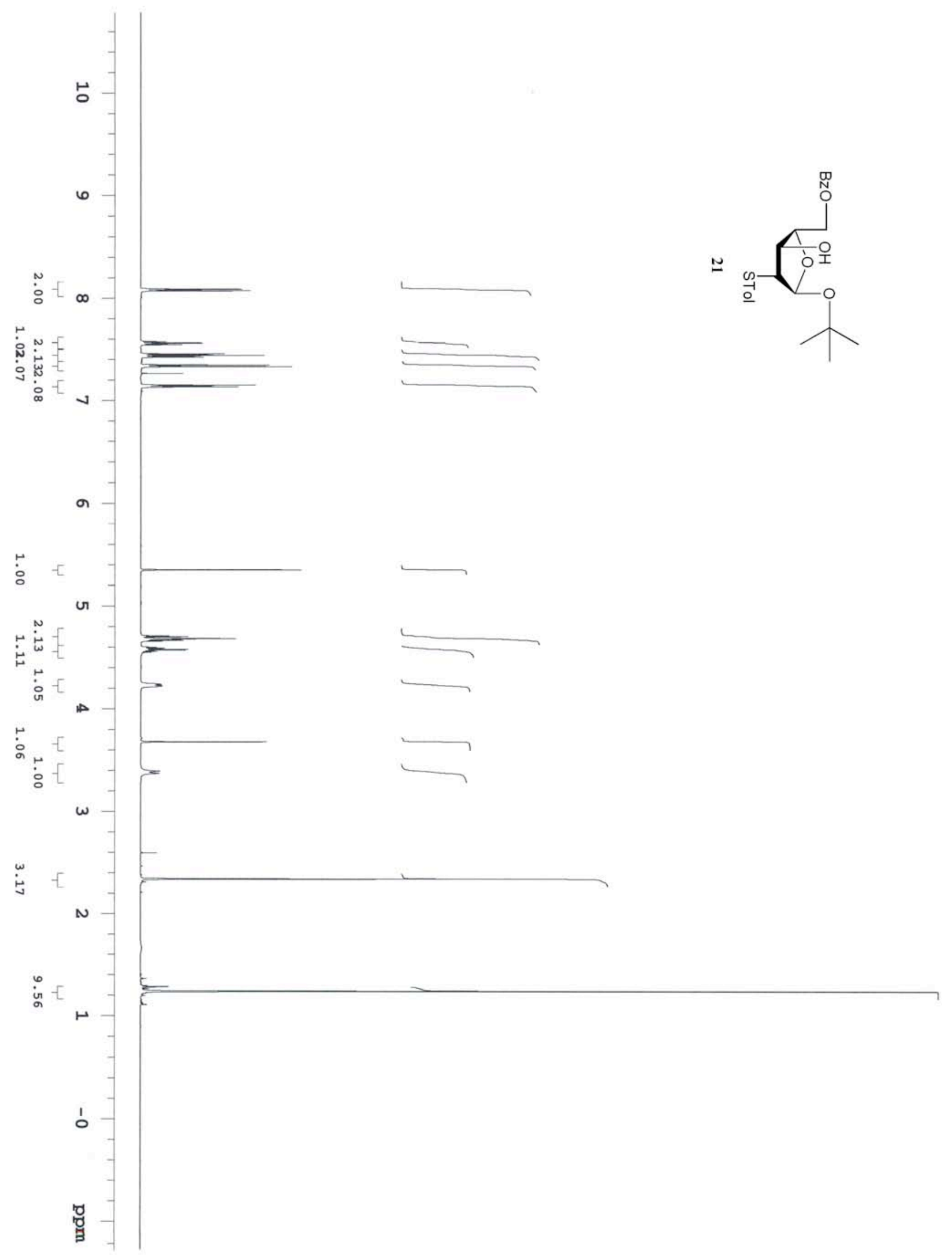




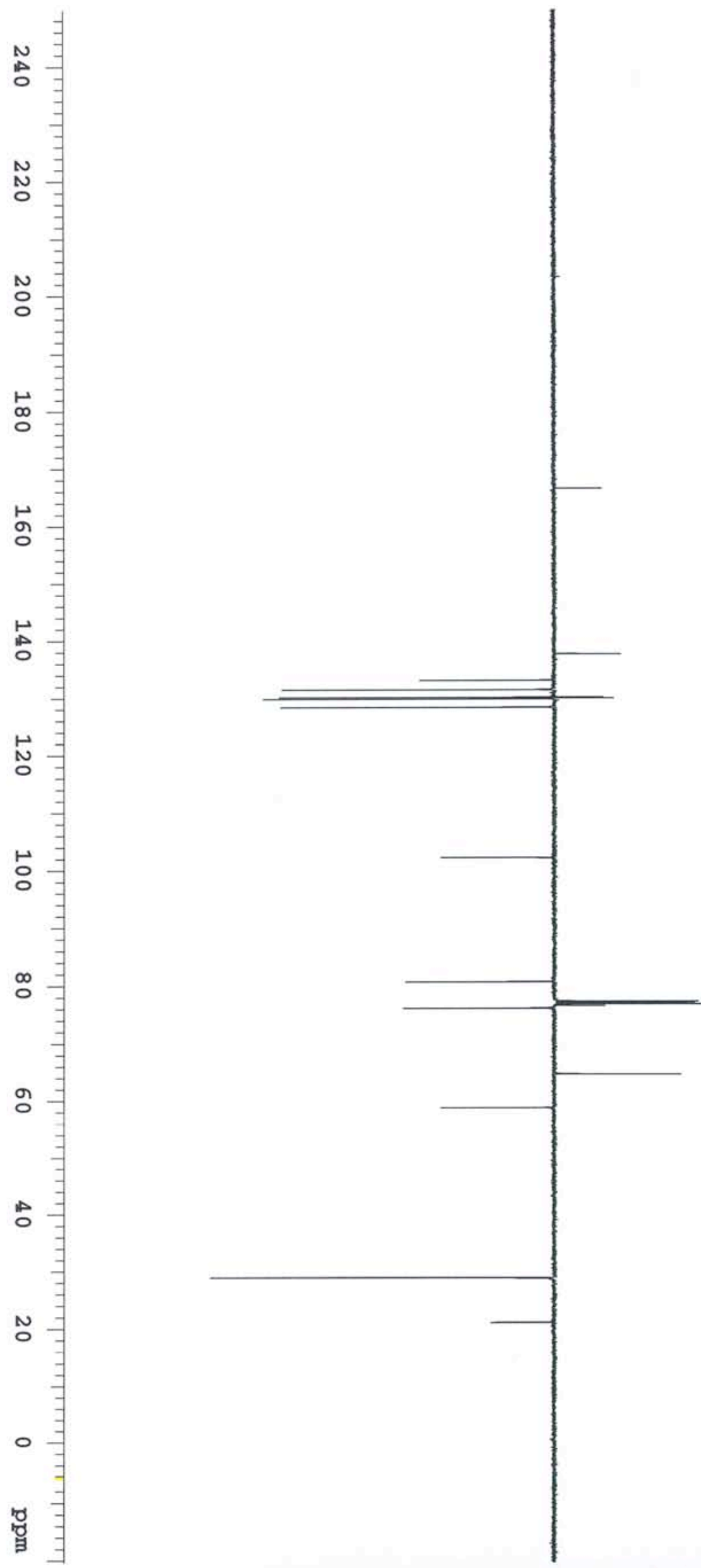

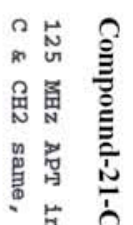

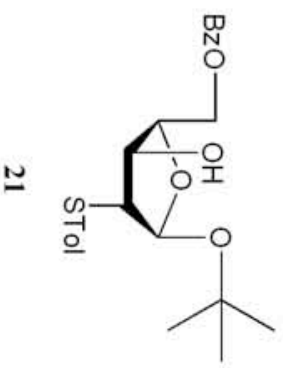

몸ำ

웅

兽

总

政

㤎点

品

骂:

筧

166.415

㟧罾
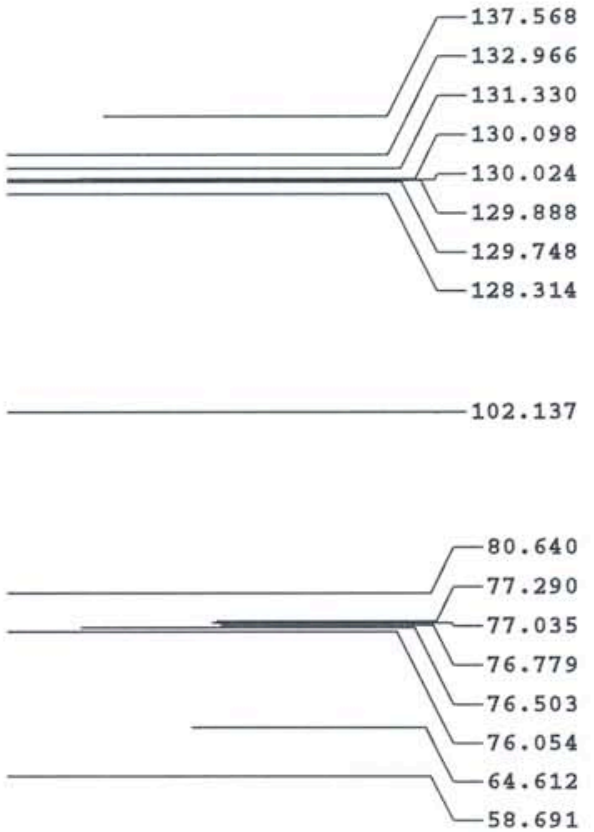

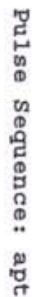




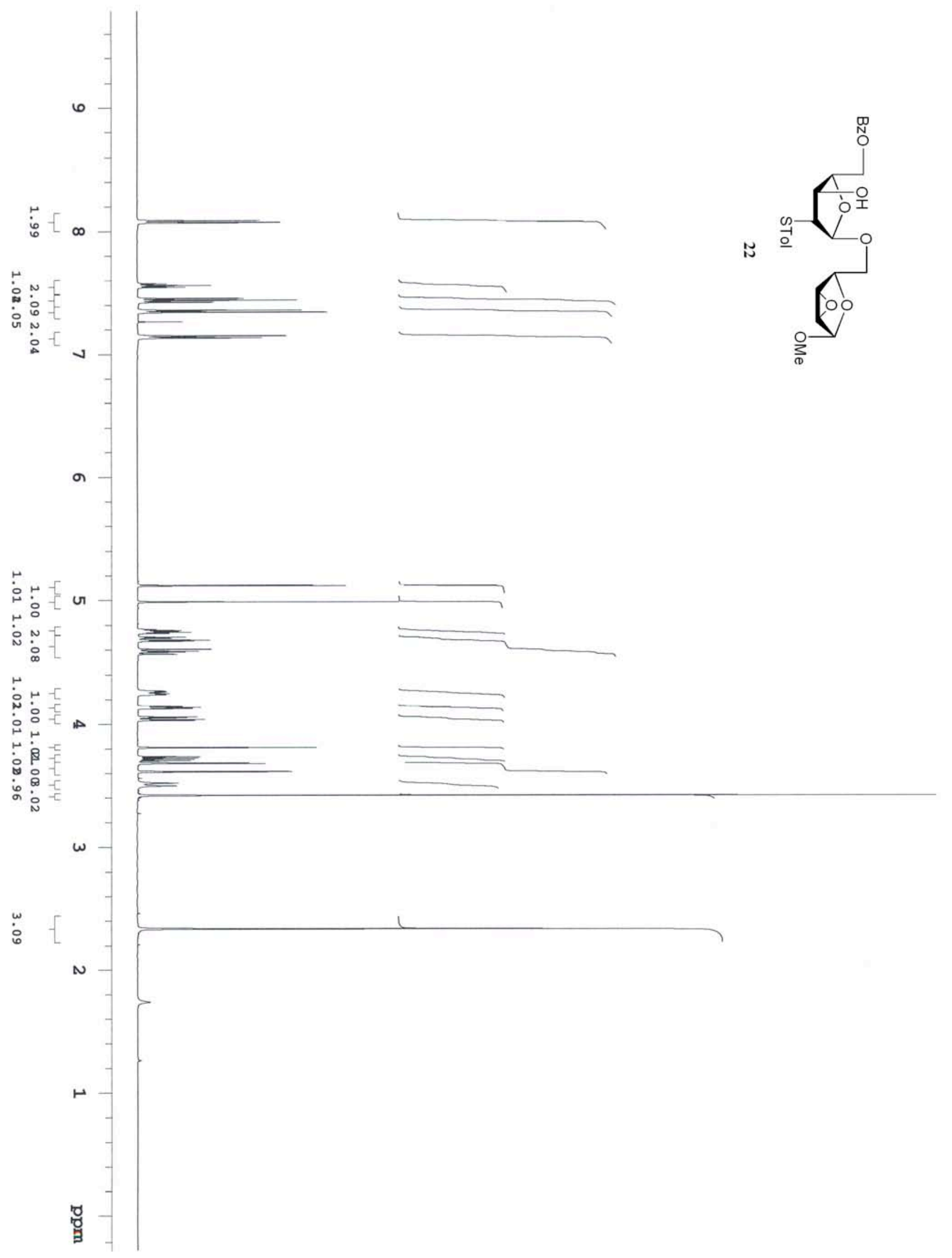




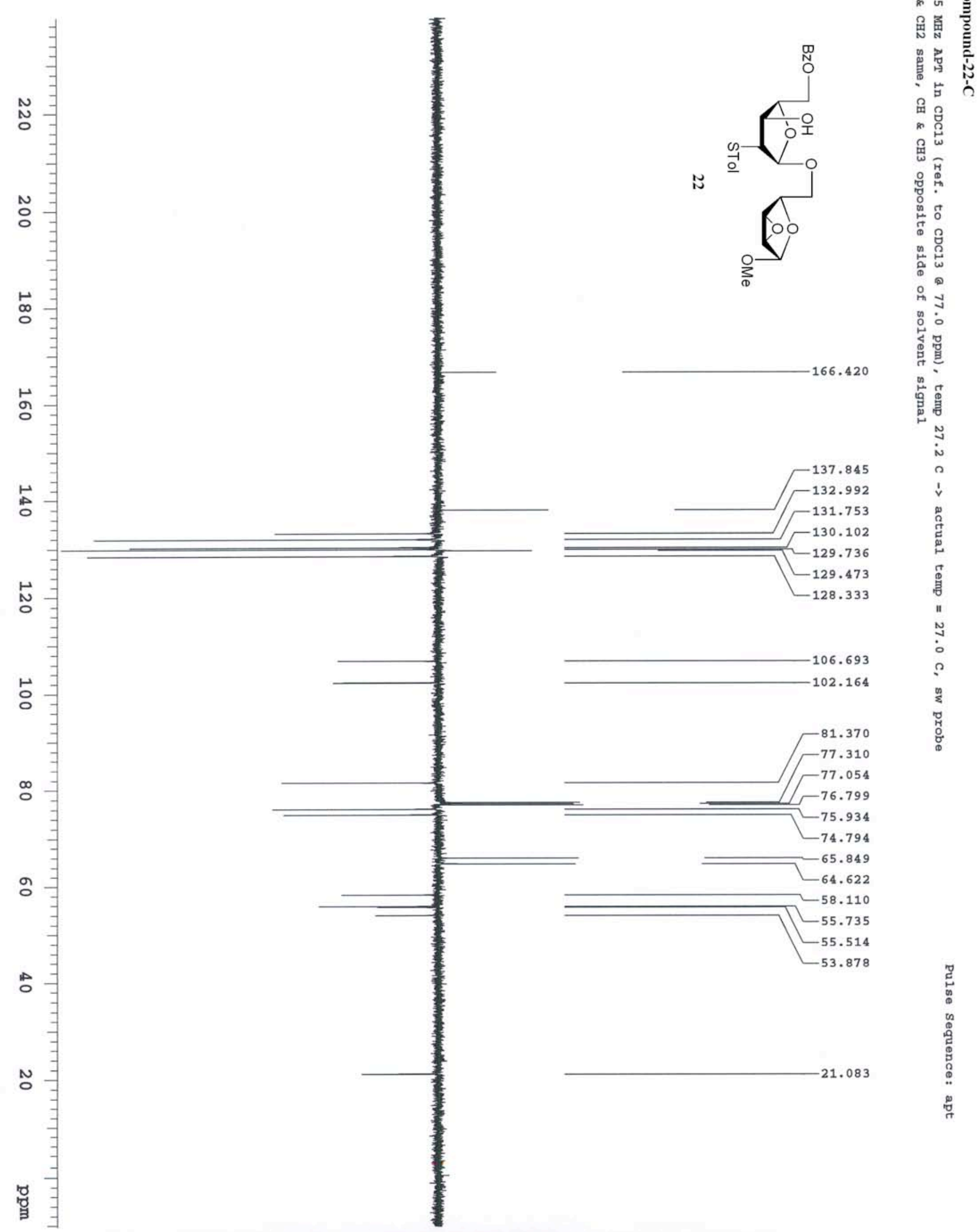




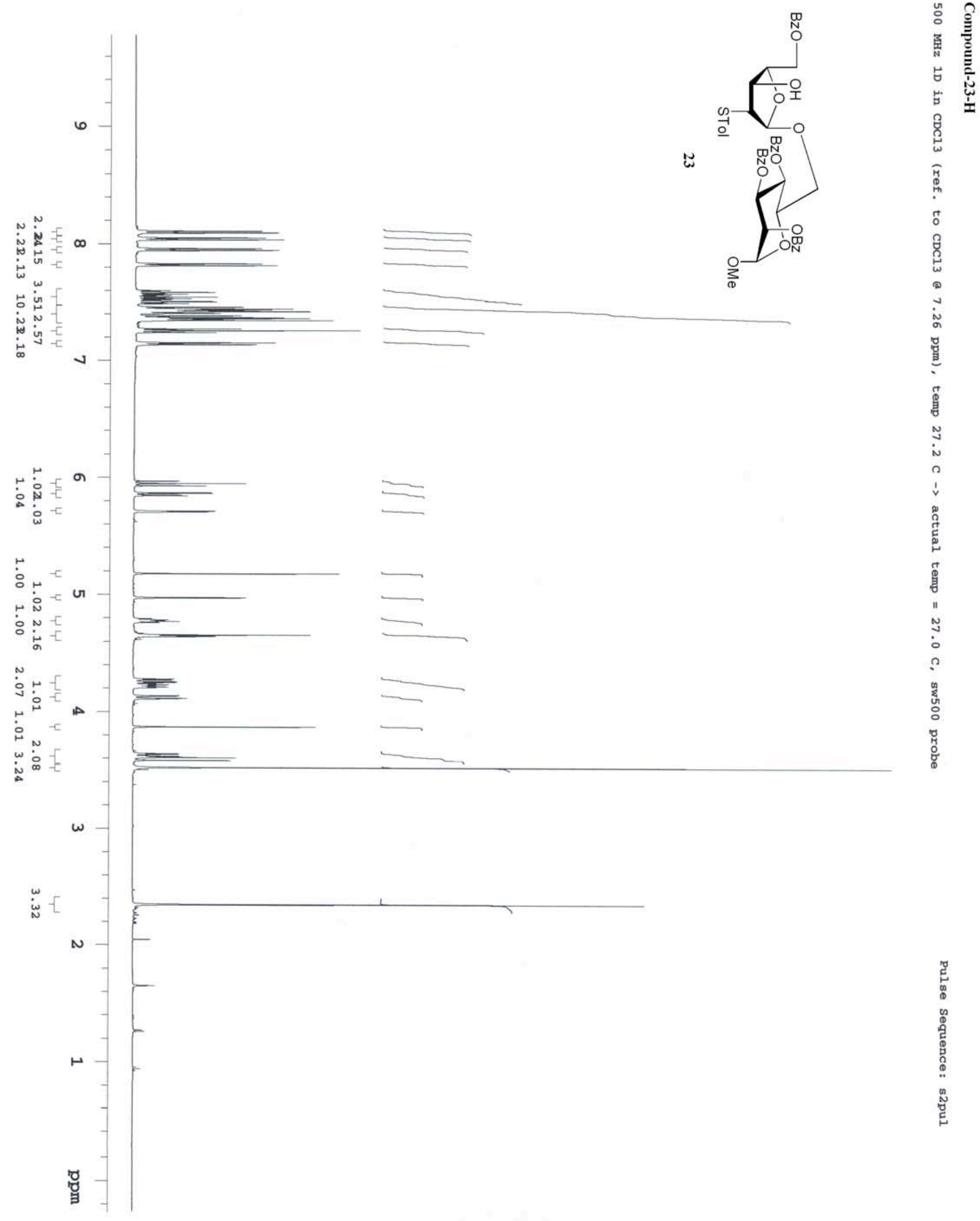



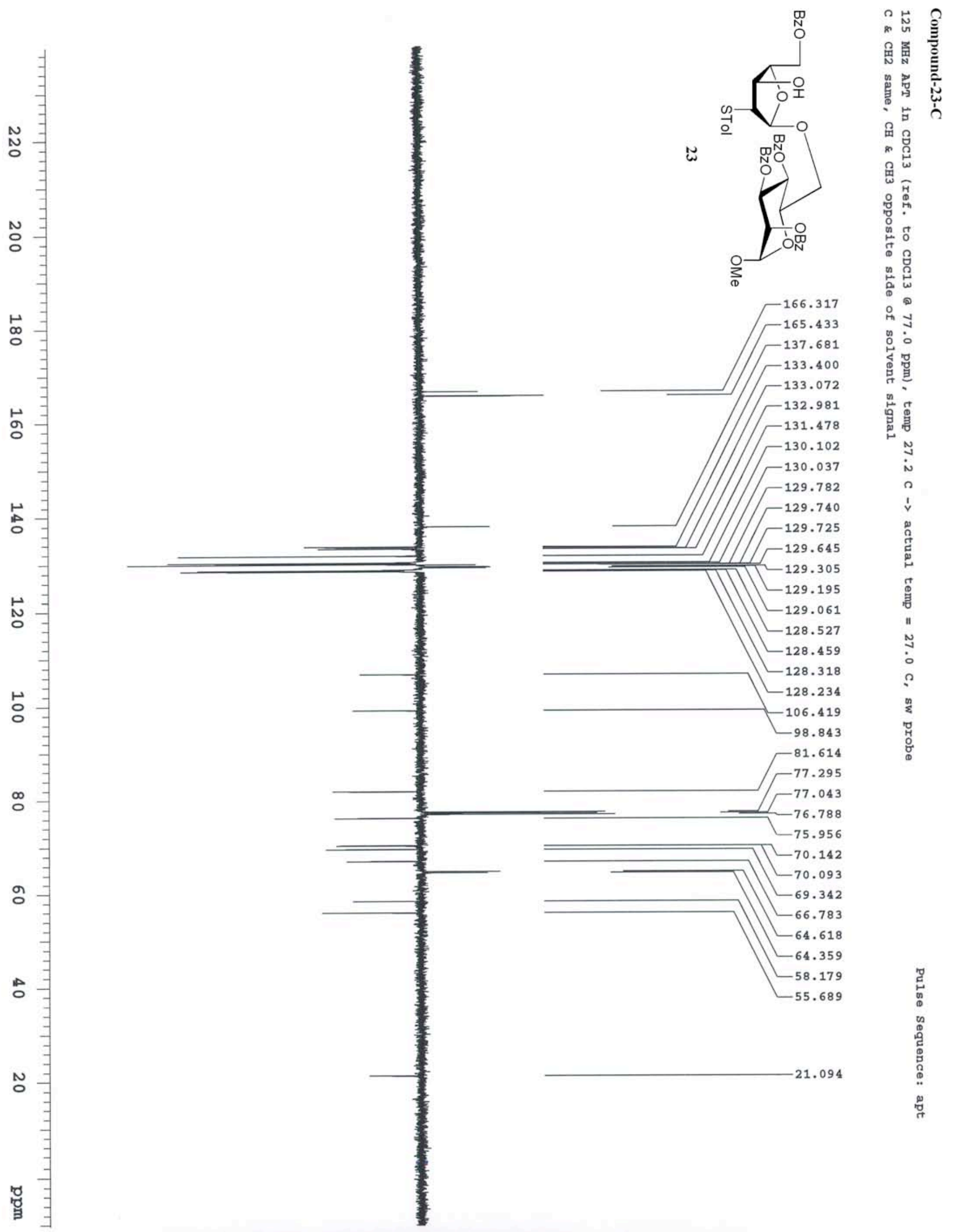


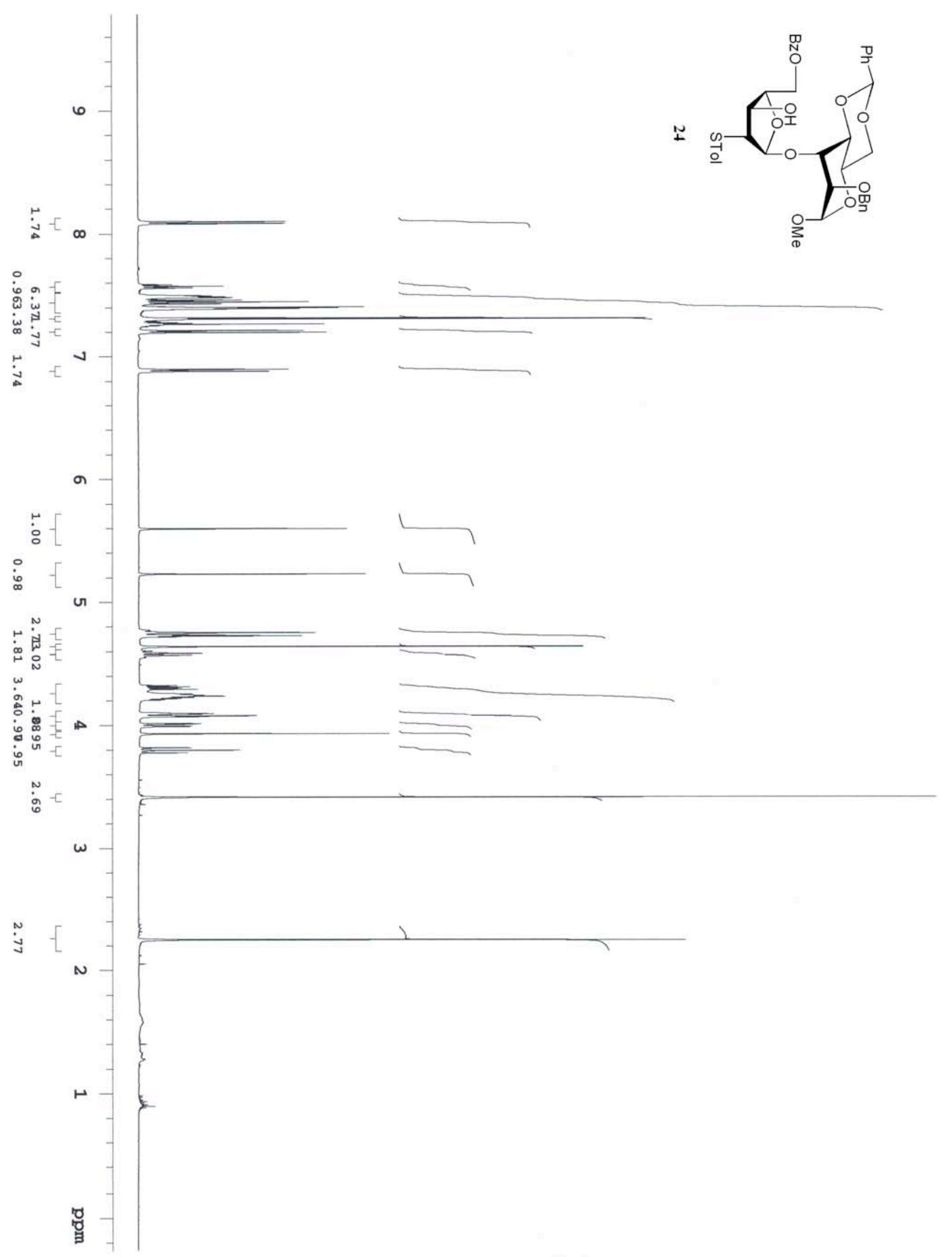




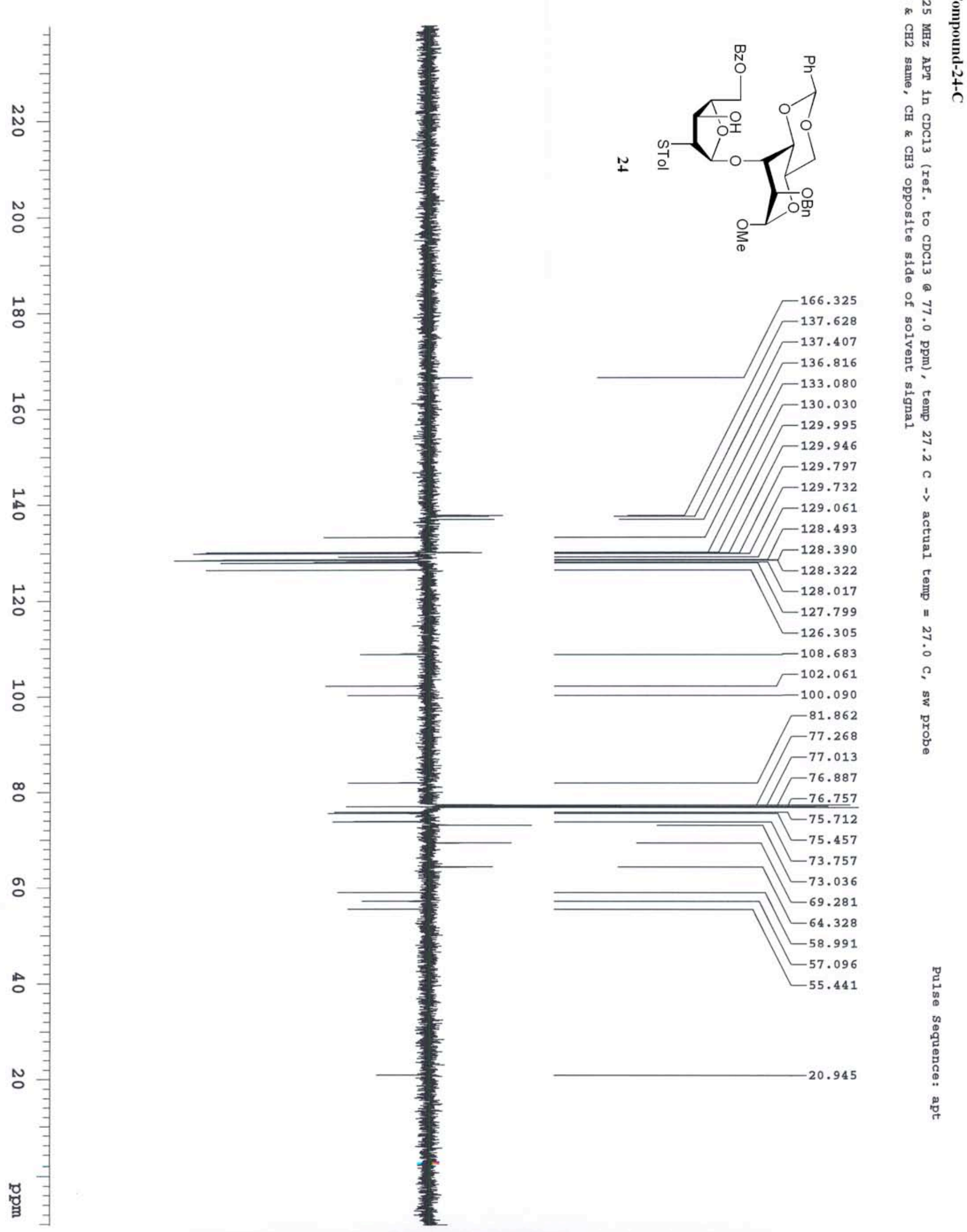




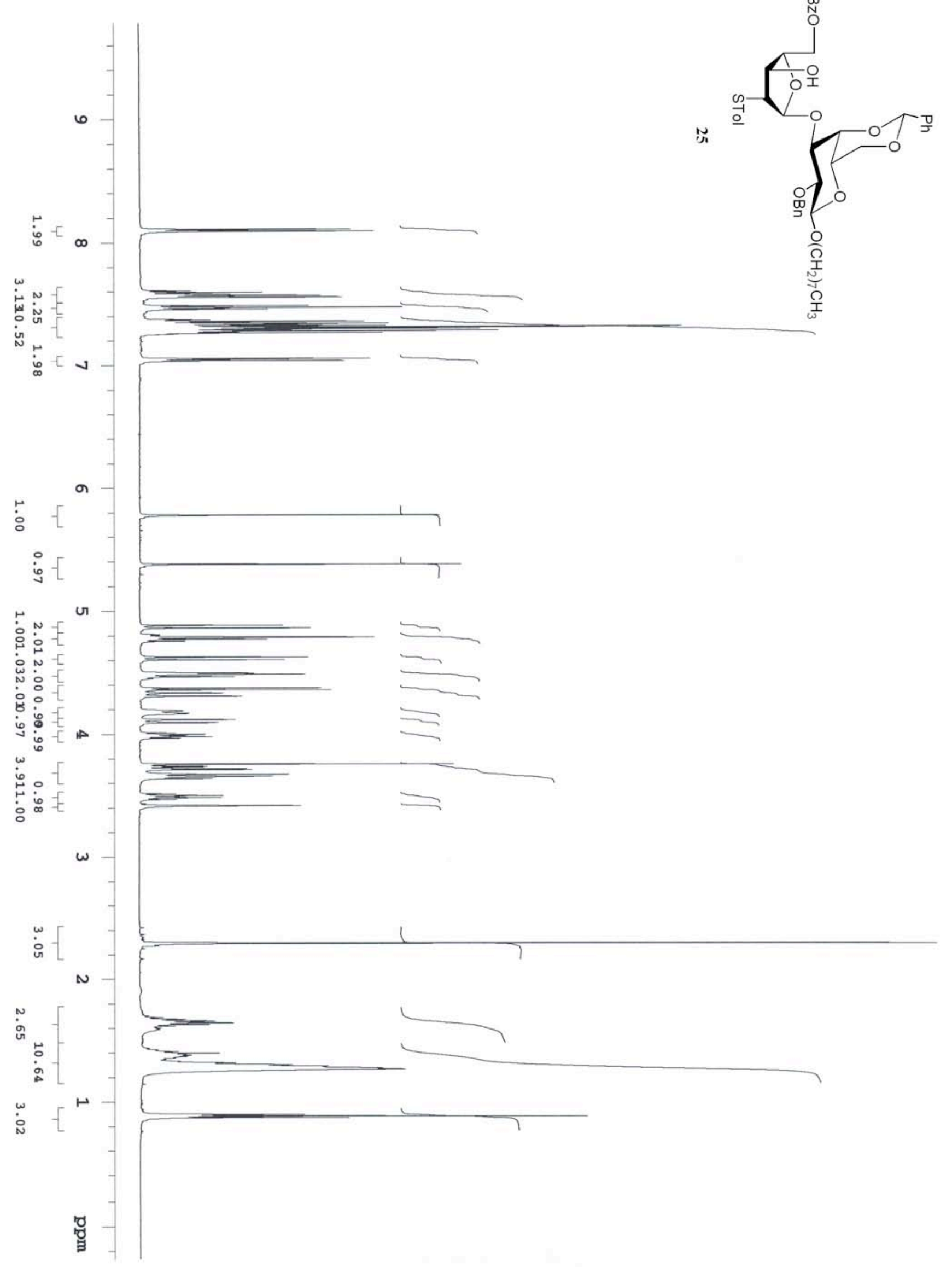



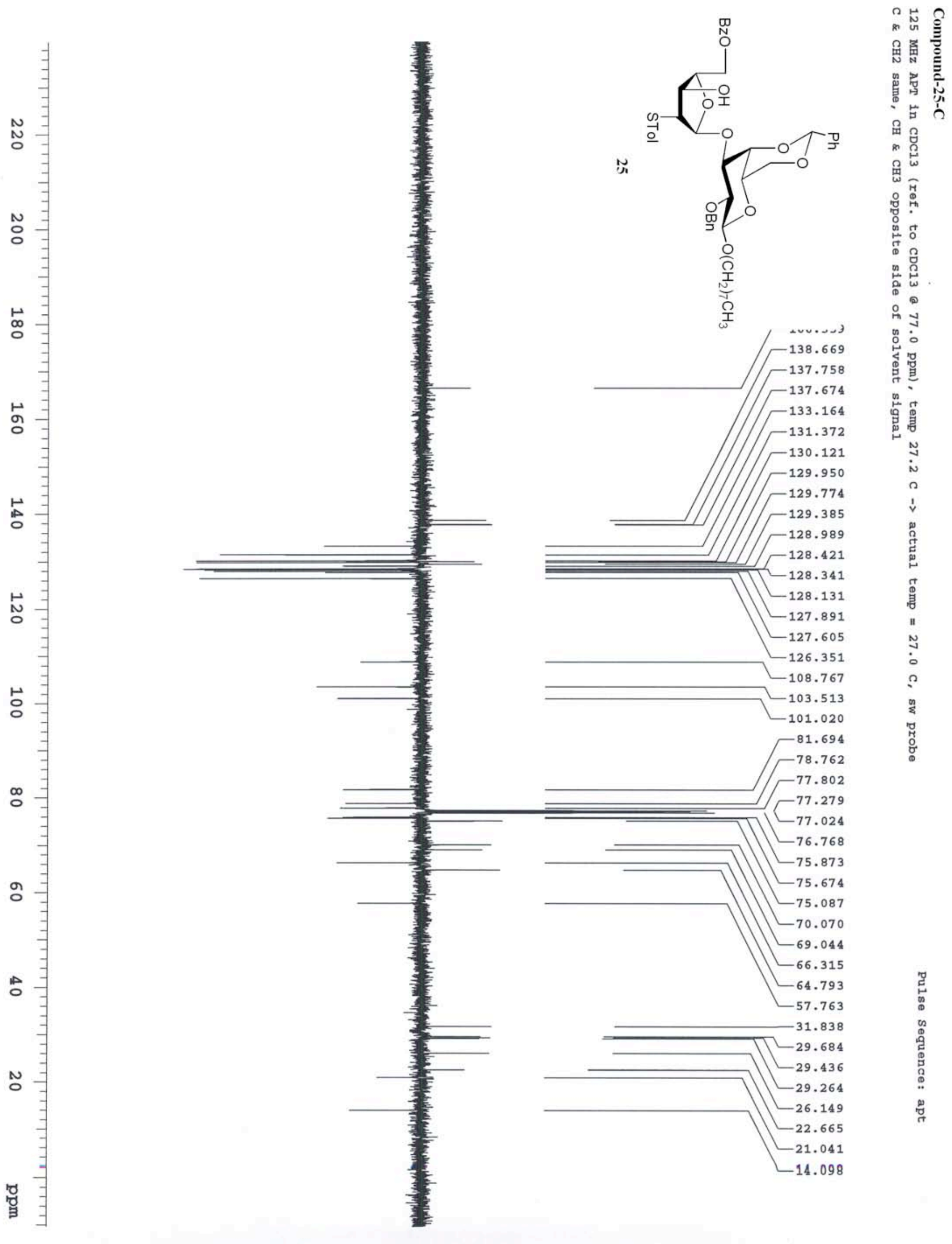


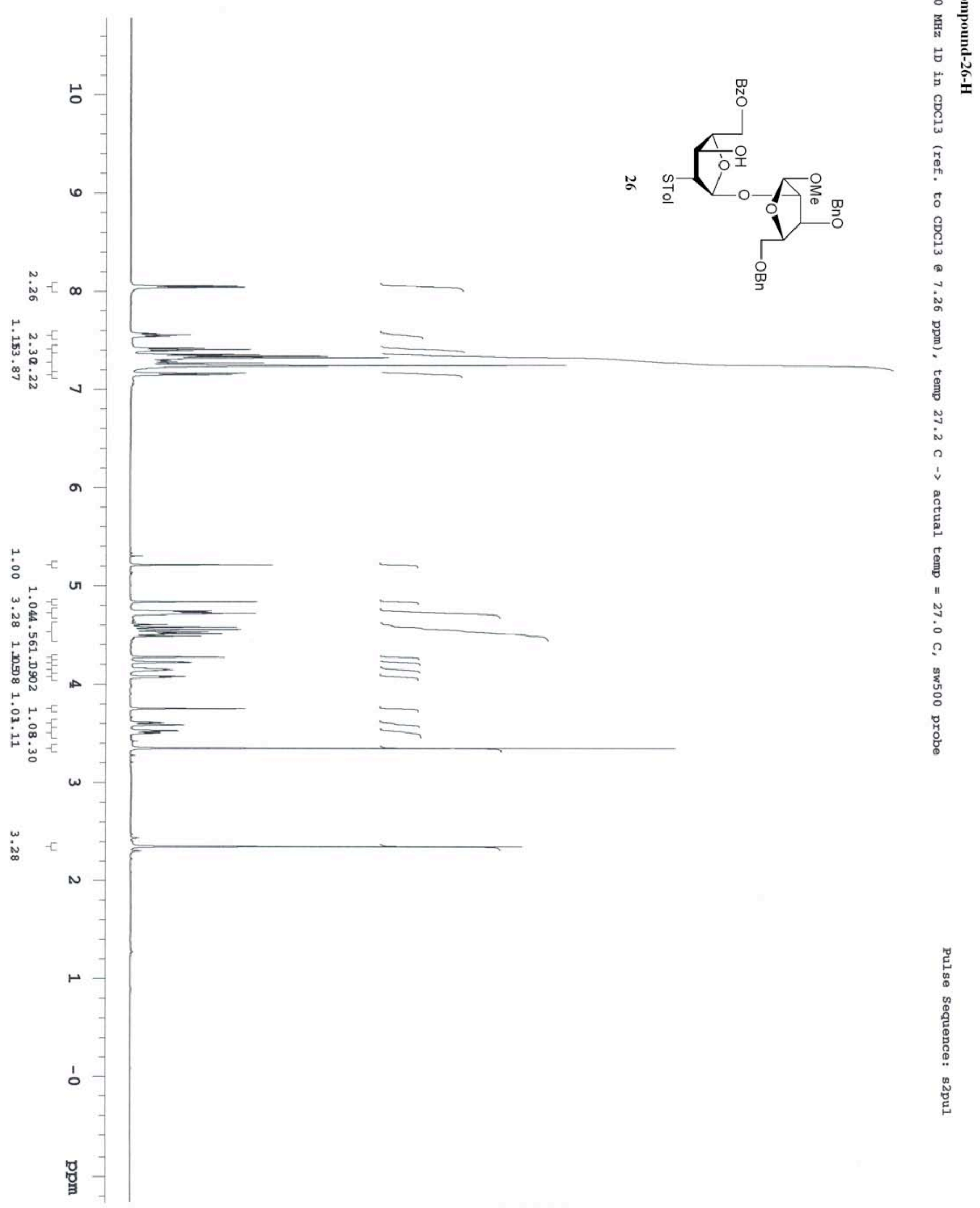



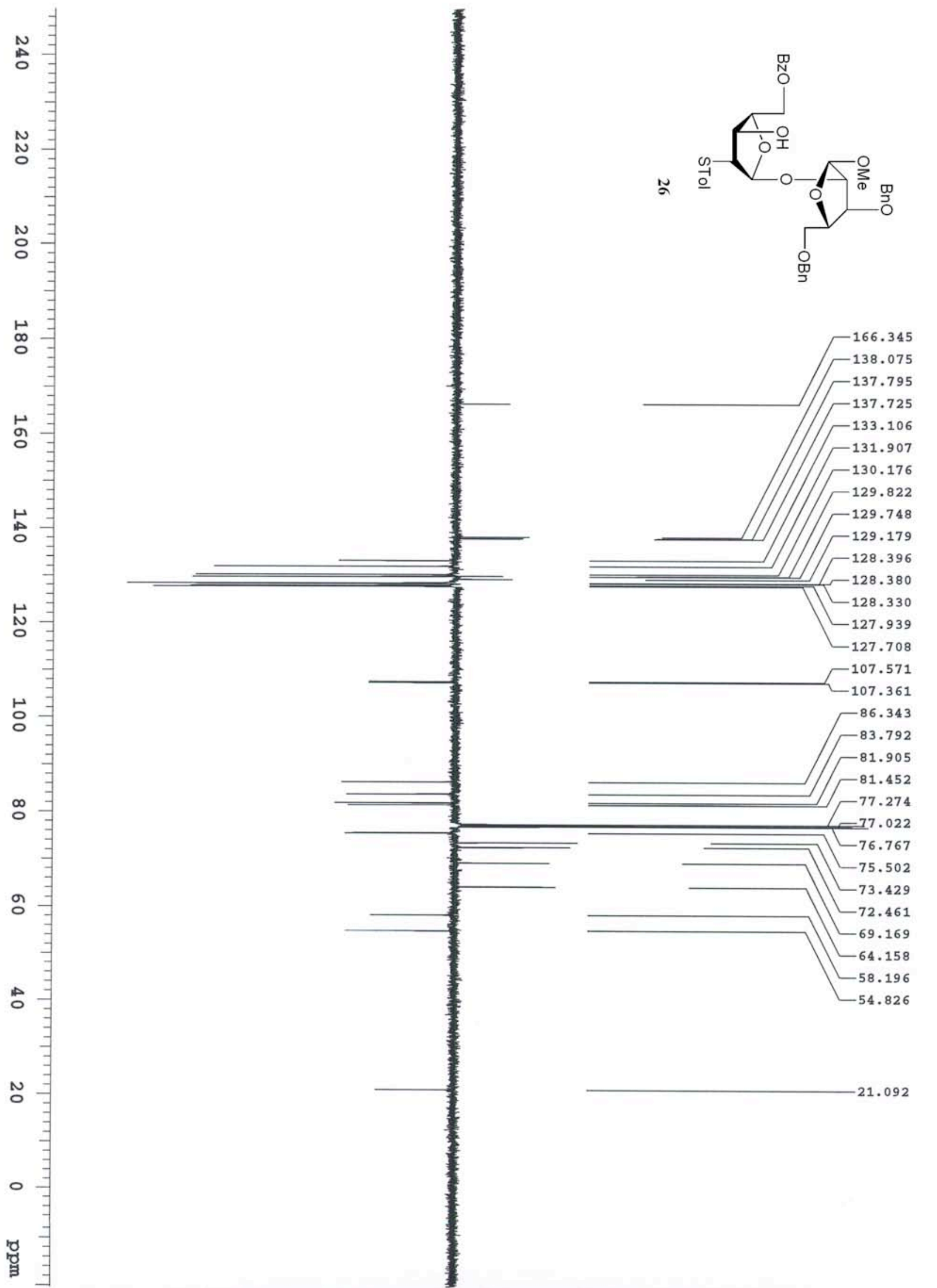

ก 崩

贸息

宸

范

品

苗

क

:ㅇํำ

क्ष

(t)

恕葛

ำ

का

管

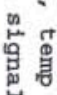

$-133.106$

$-131.907$

$-129.748$

$-129.179$

$-128.396$

$-127.939$

$-127.708$

$-107.571$

$-107.361$

$-86.343$

$-83.792$

$-81.905$

$-81.452$

$-77.022$

$-76.767$

$-75.502$

$-73.429$

$-72.461$

$-64.158$

$-58.196$

$-54.826$

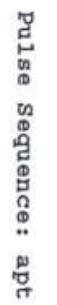




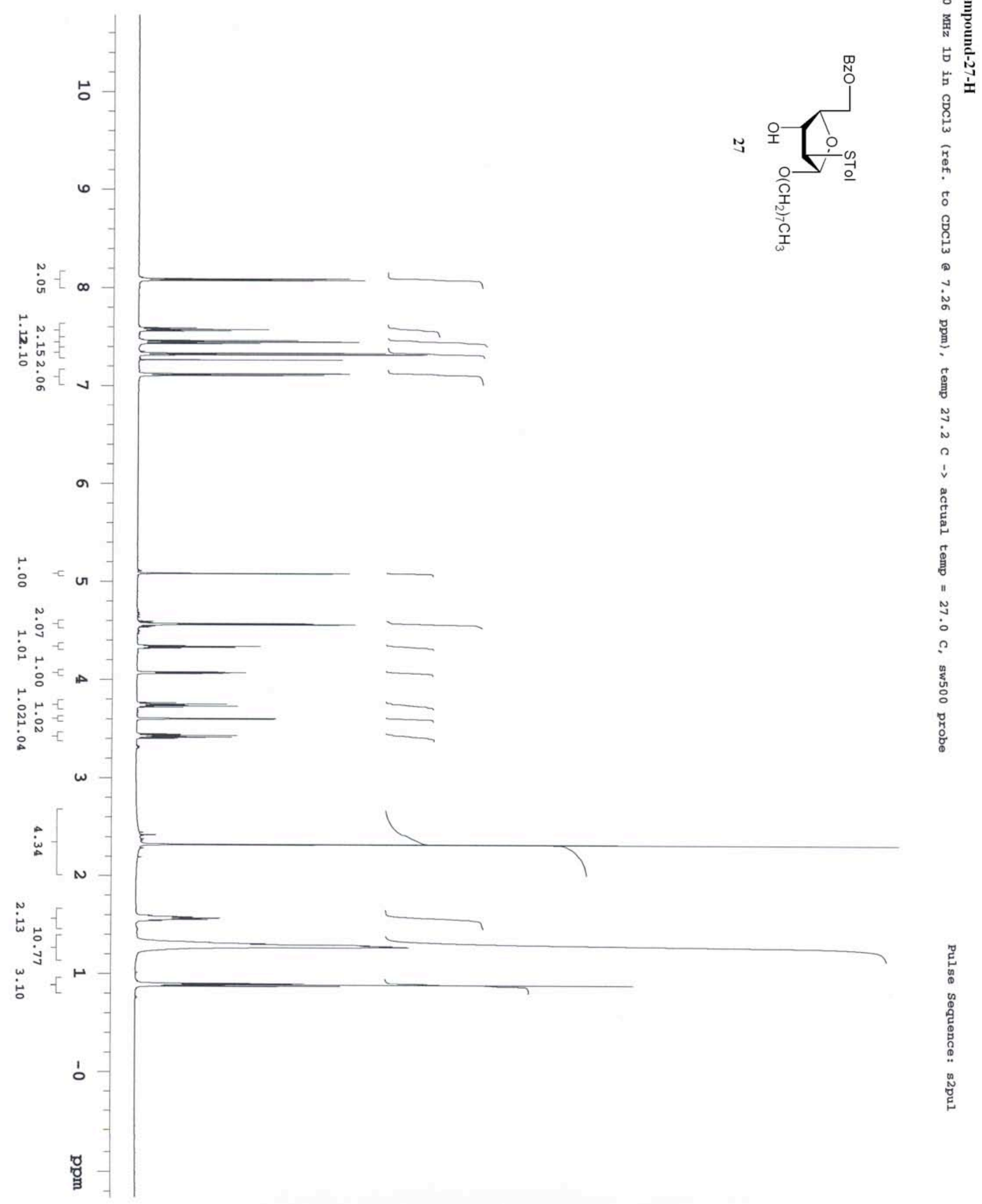




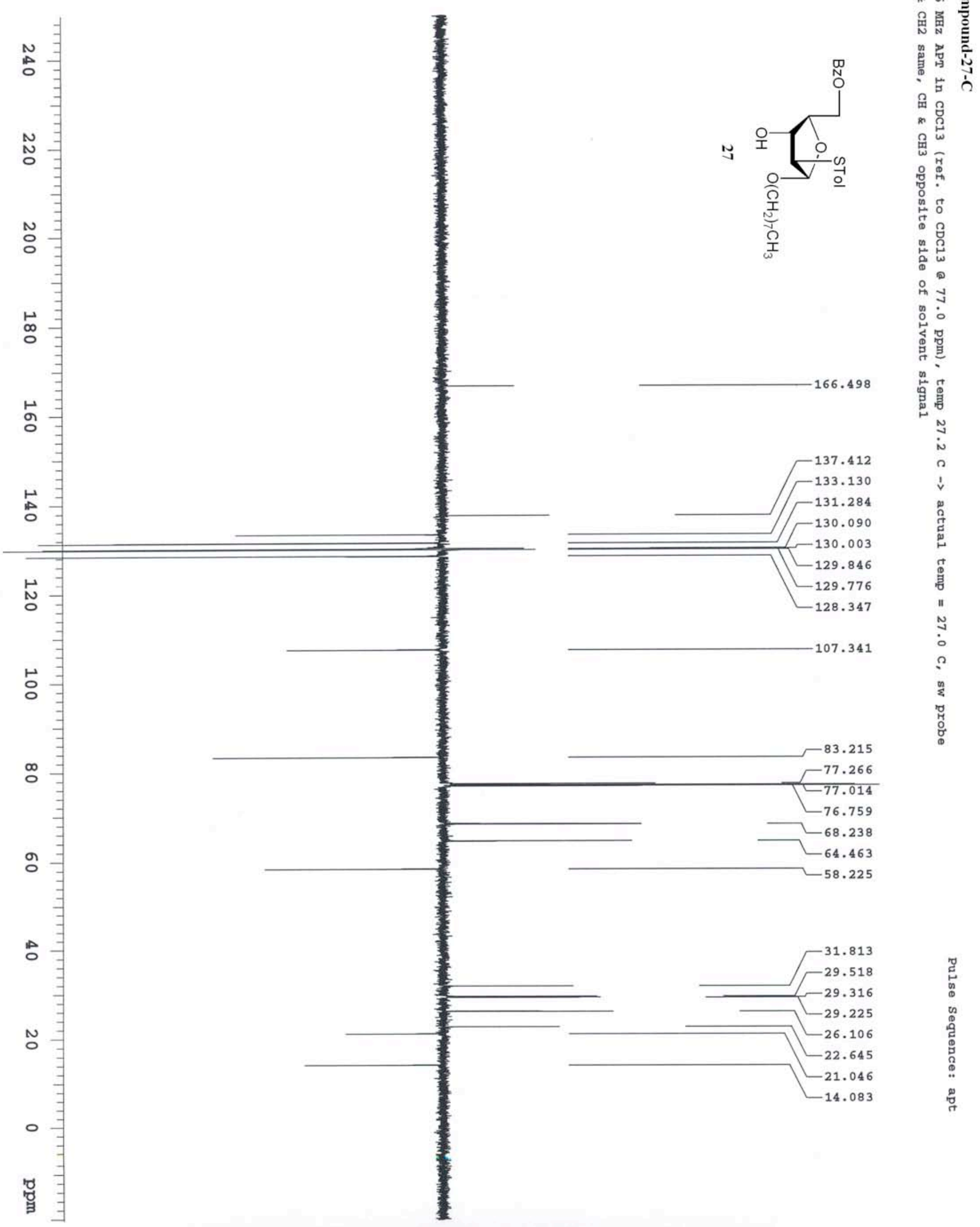




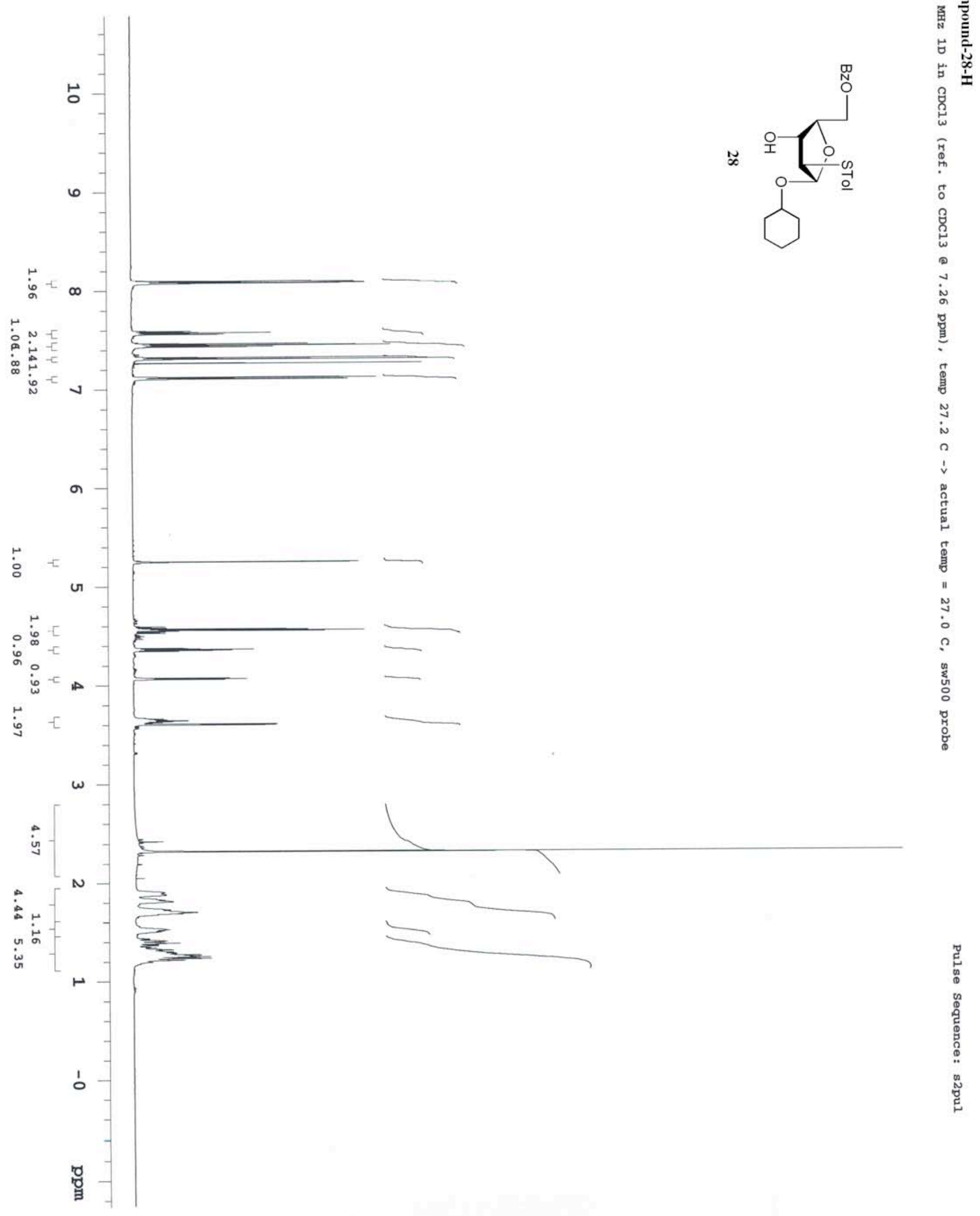



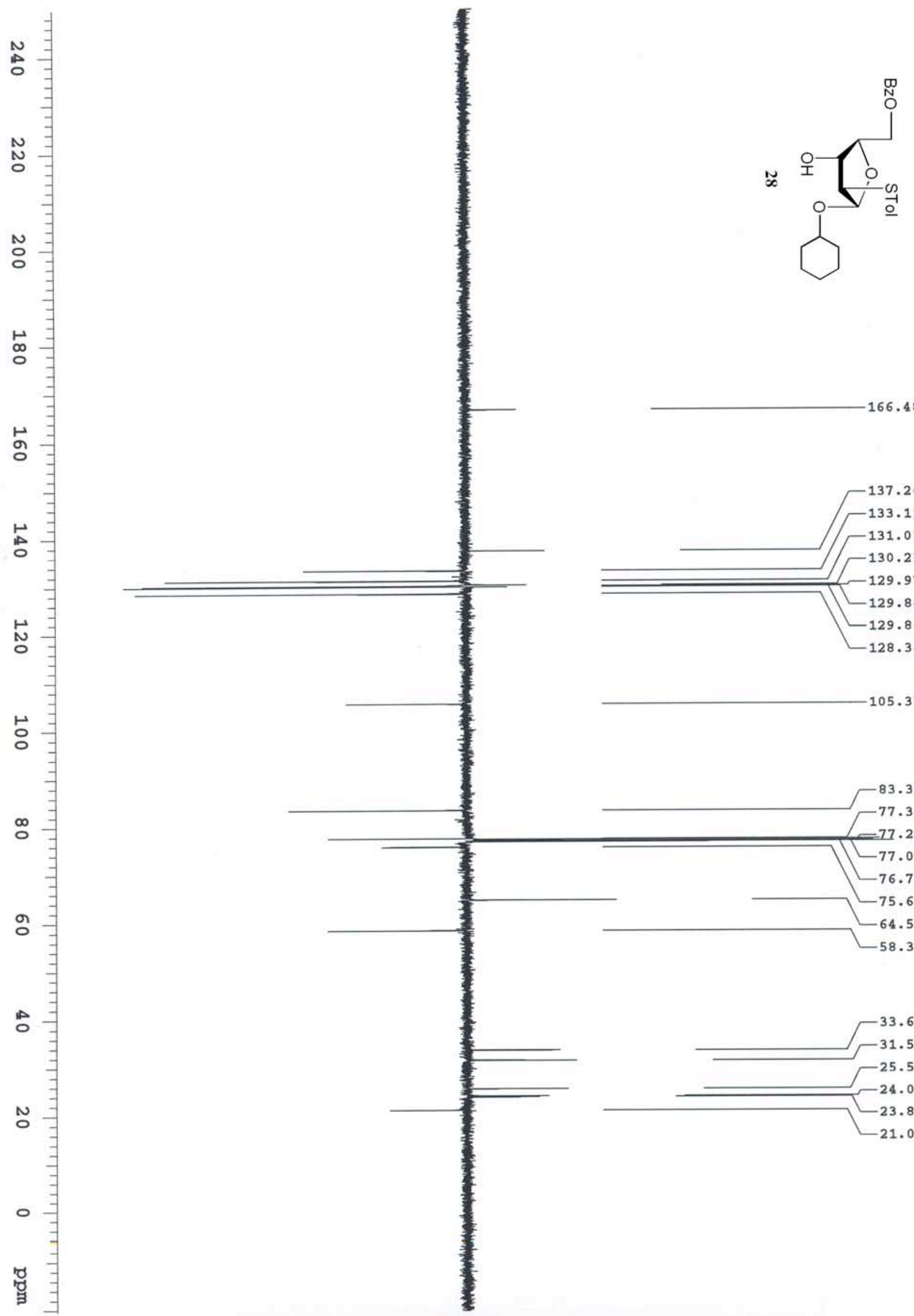

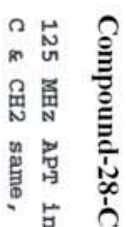

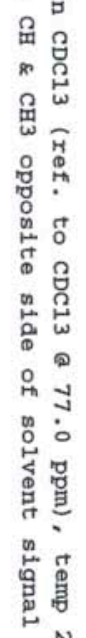

$-166.481$

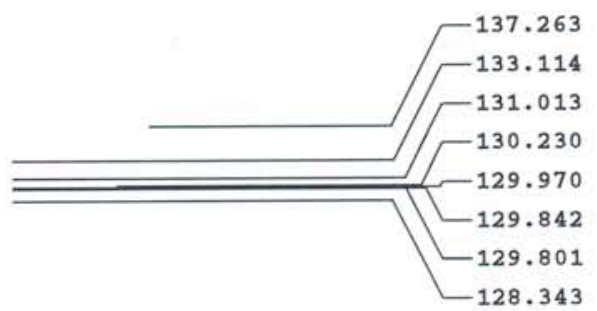

$-105.379$

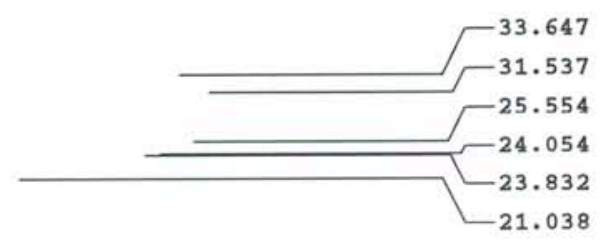




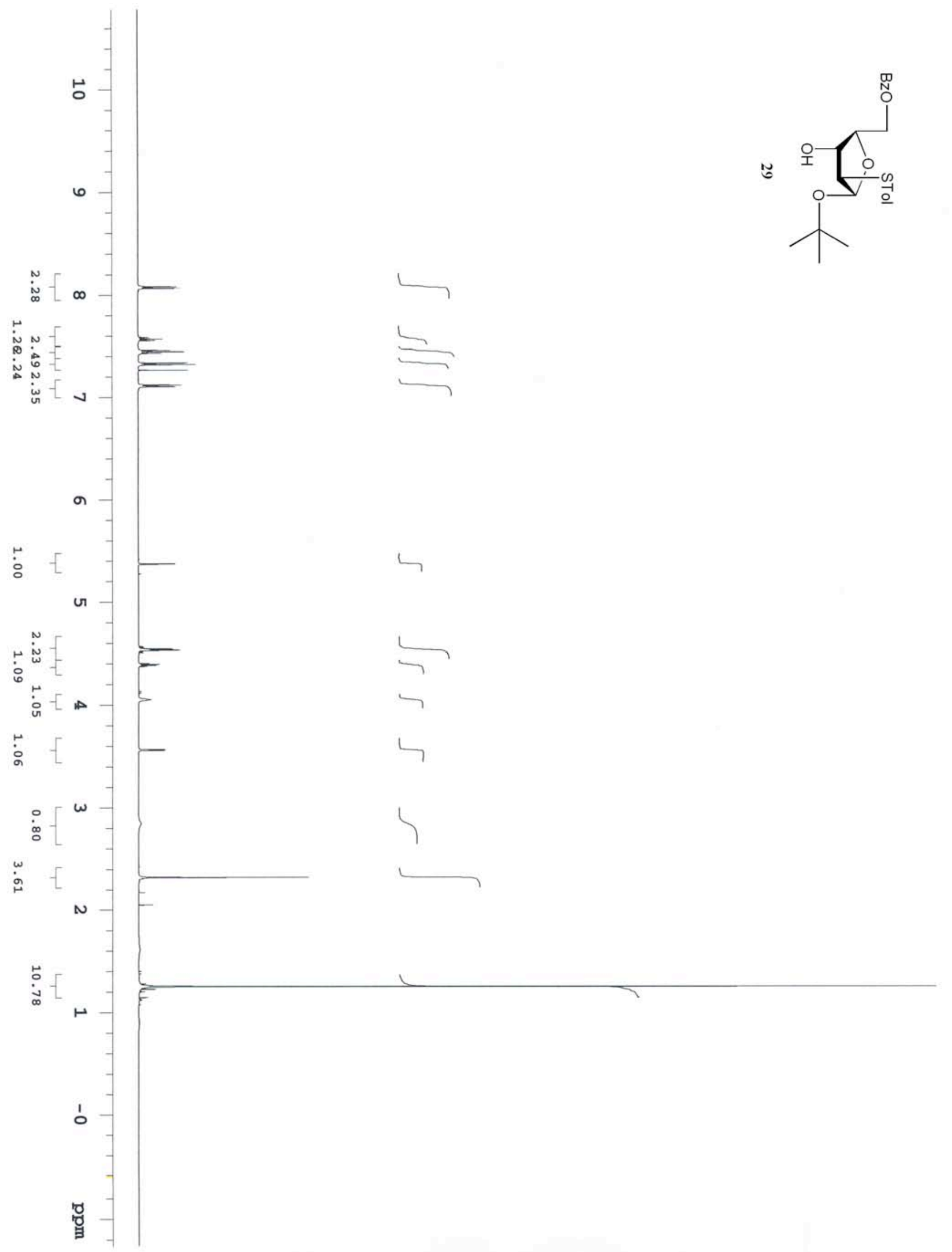

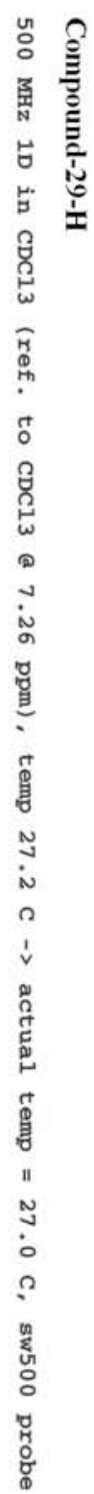

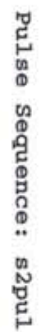




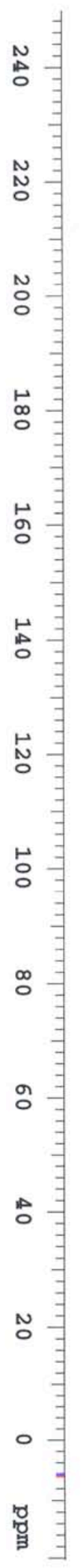

م $\underset{N}{\stackrel{N}{N}}$

居

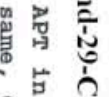

㽞?

n

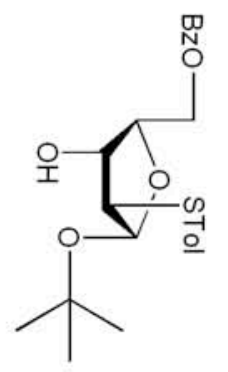

$-166.461$

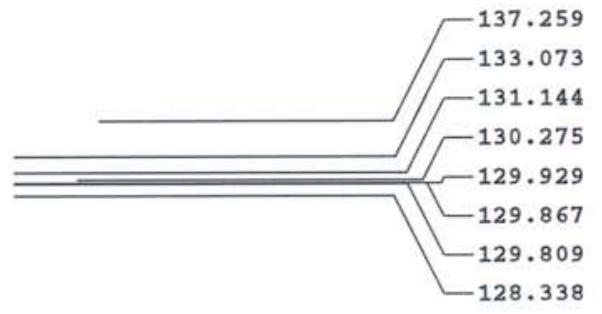

"

罚

움

28.723 


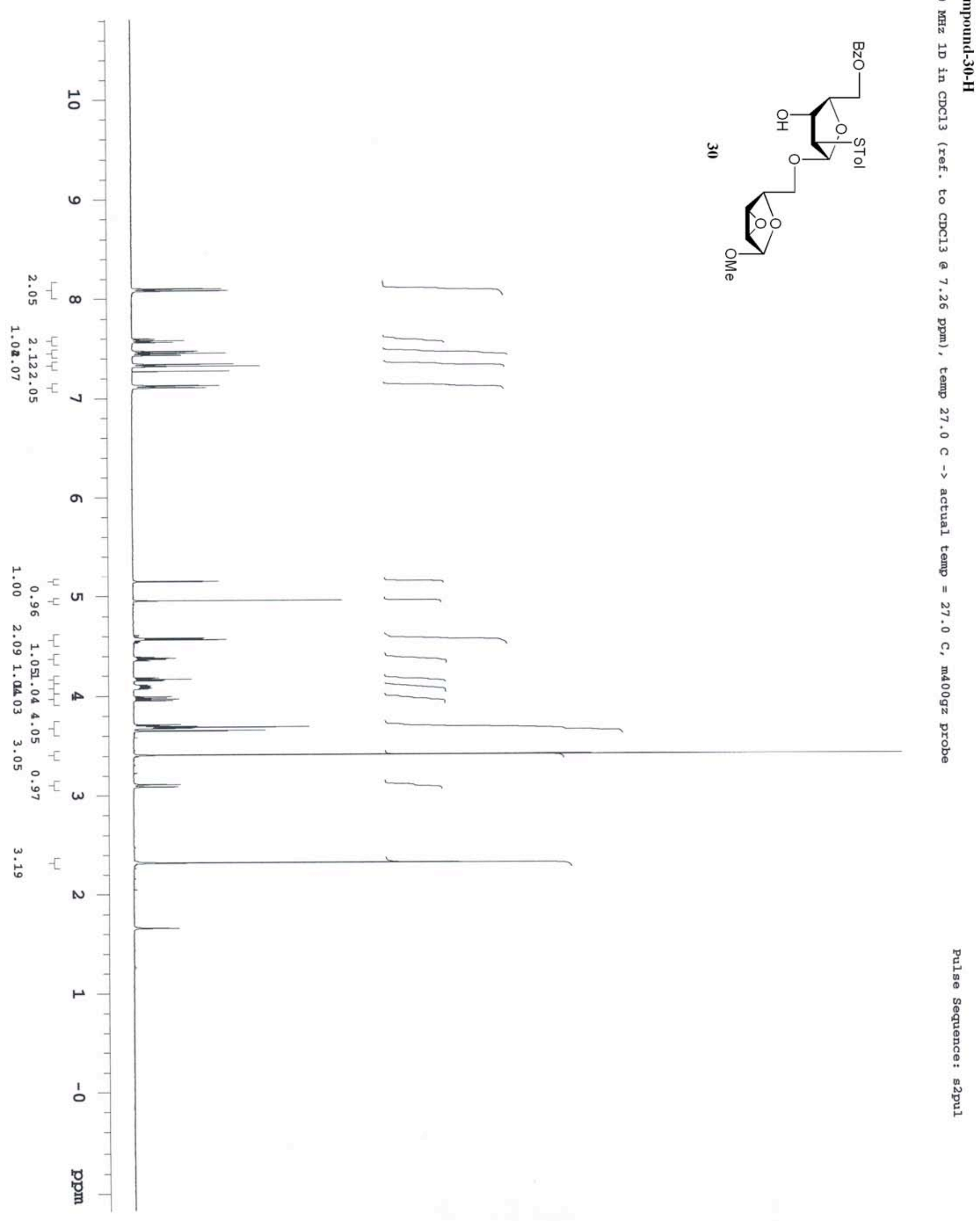




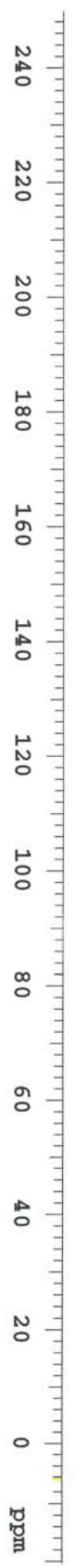

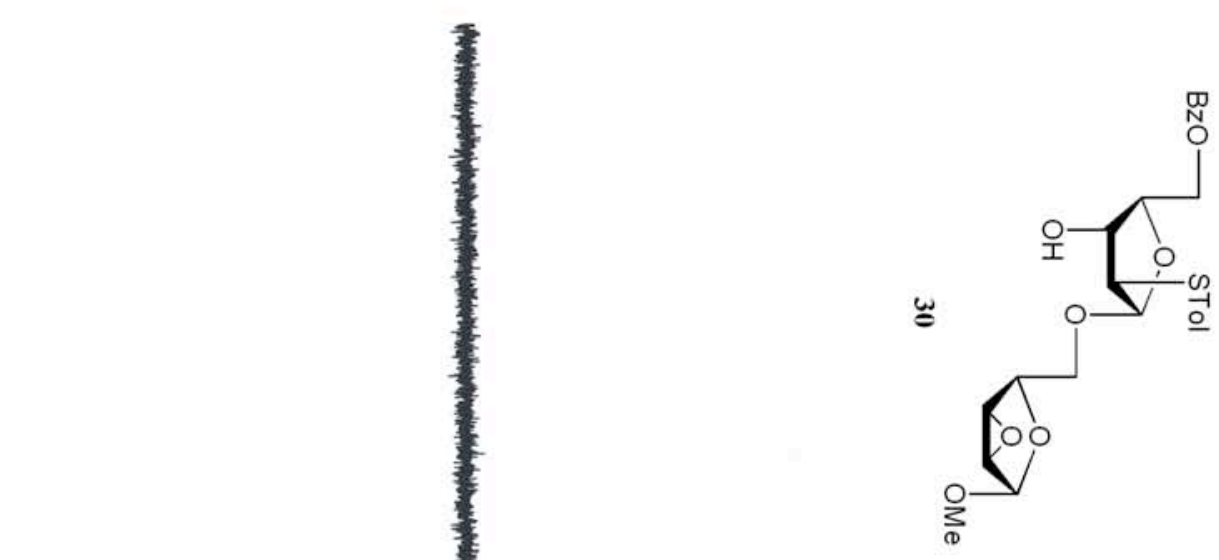

舟芯

蛋惫

筧总言

药

㽞?

喝

웄

覀

(2)

응

क

雚

ㅇำ

:

䀦

$-166.465$

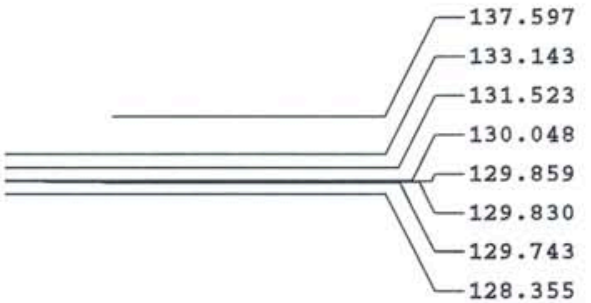

107.221

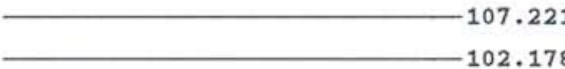

$-83.809$

$-77.319$

$-77.261$

$-77.010$

$-76.750$

$-74.58$

$-65.419$

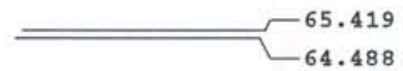

$-58.303$

$-56.082$

$-55.596$

$-53.964$

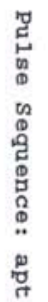




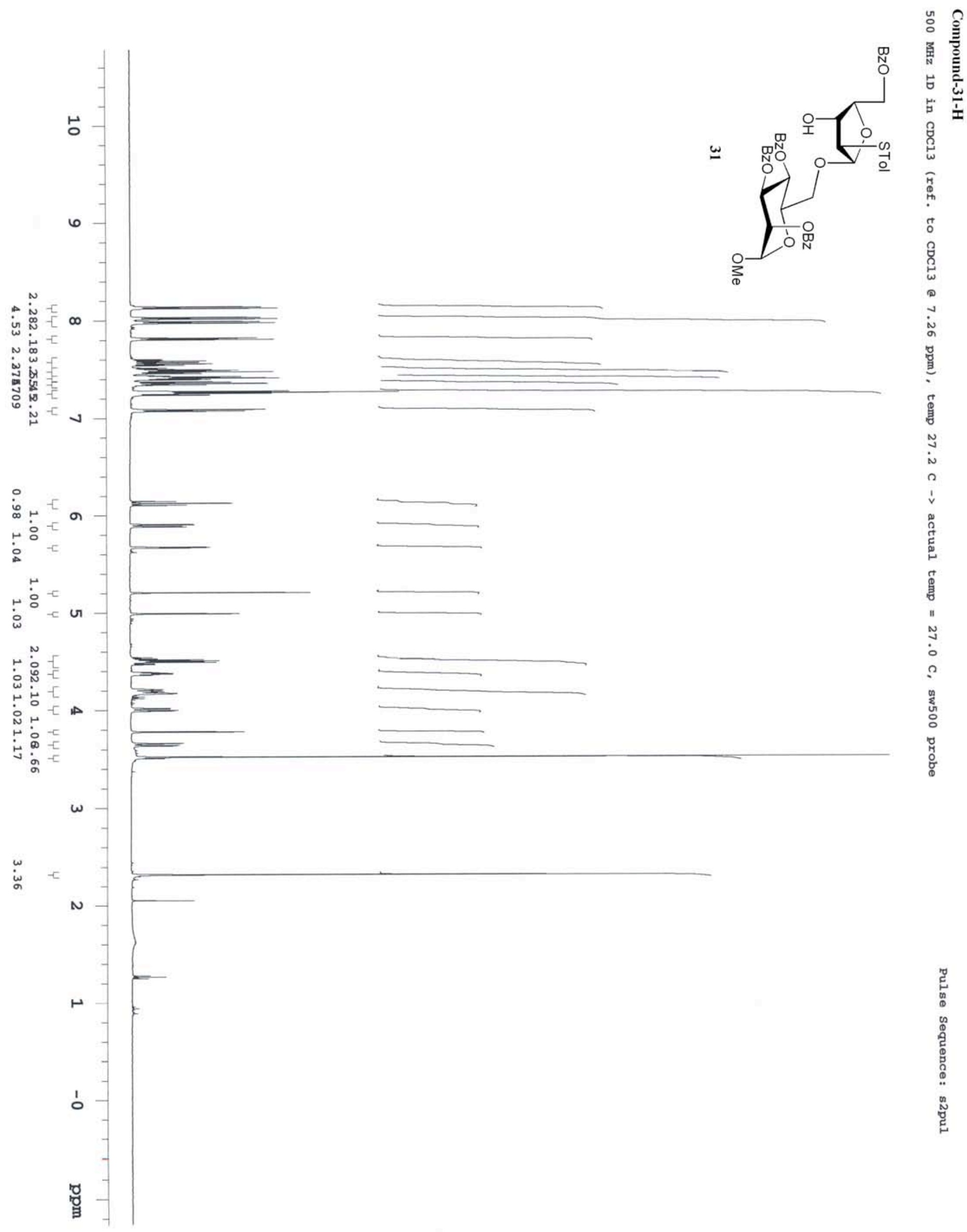



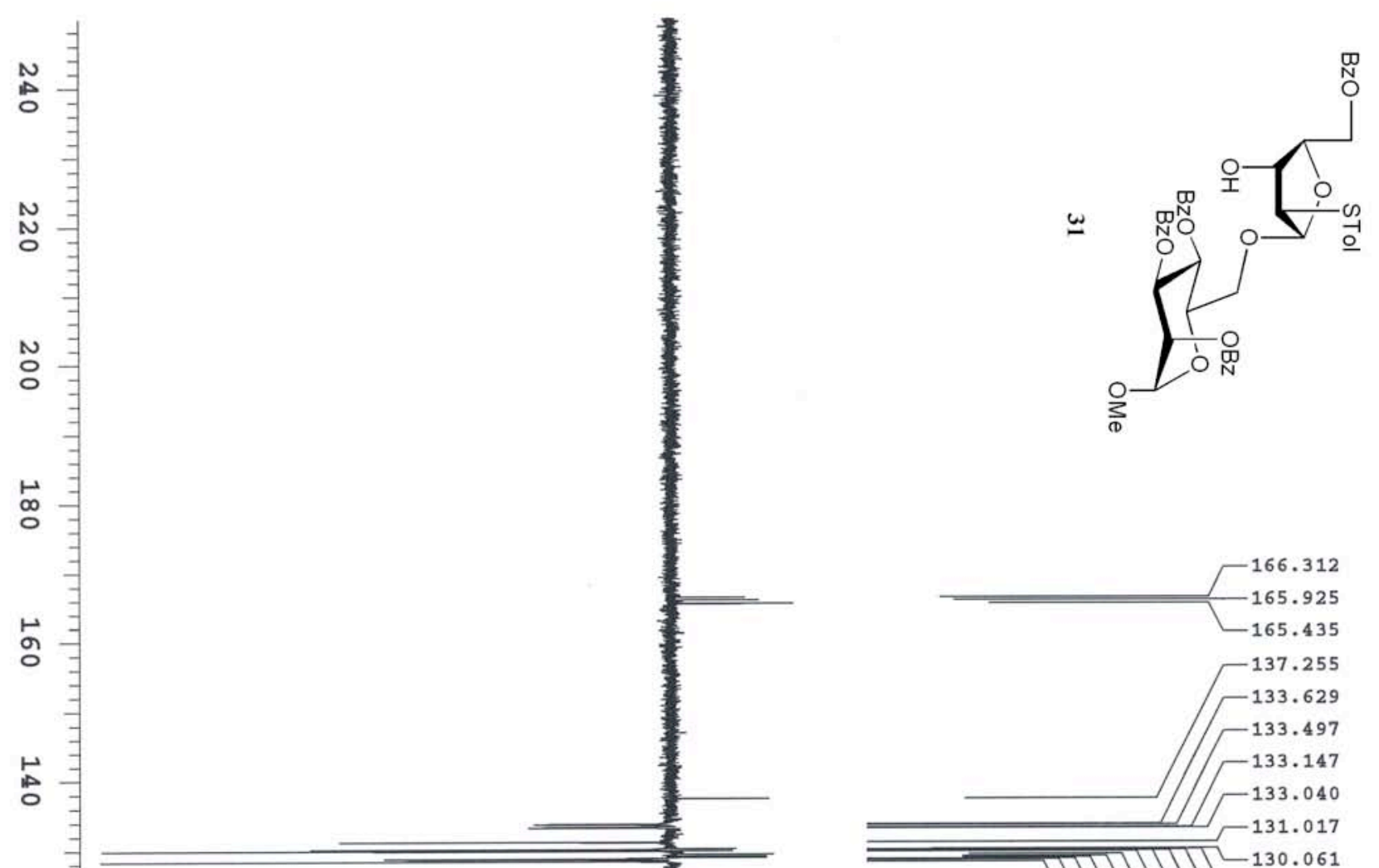

突

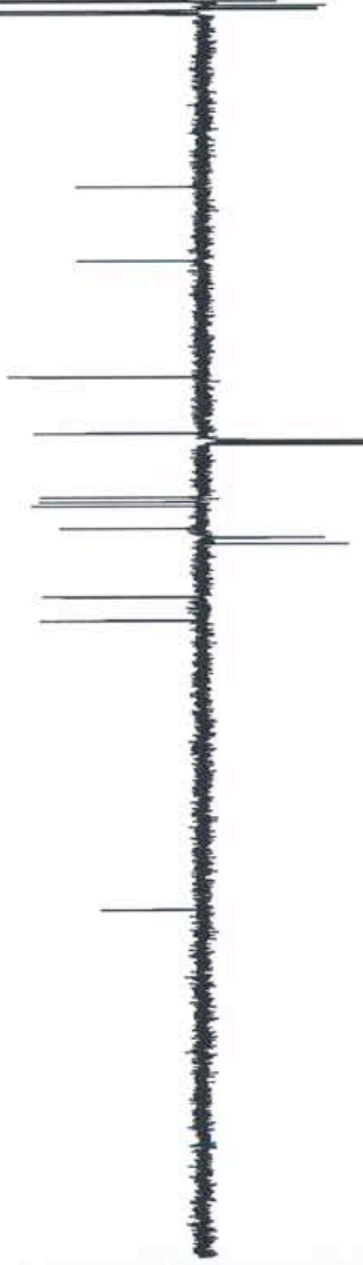

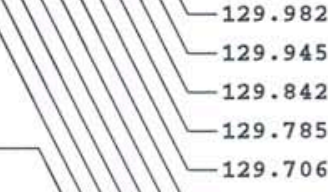

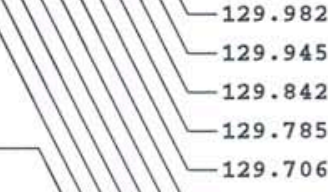

농

官

$\stackrel{\infty}{\circ}$

$\stackrel{\infty}{\circ}$

。

용

-

N

N

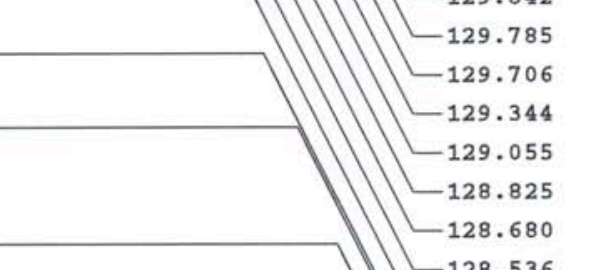

$-129.344$
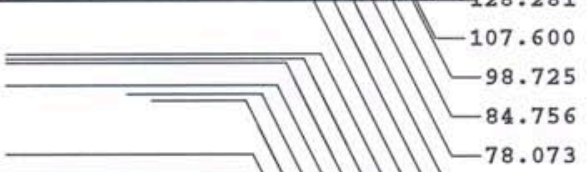

$-77.27$

$-77.018$

$-76.763$

$-70.405$

$-69.857$

$-69.346$

$-66.705$

$-65.601$

$-64.863$

$-58.534$

$-55.666$

$-21.050$ 


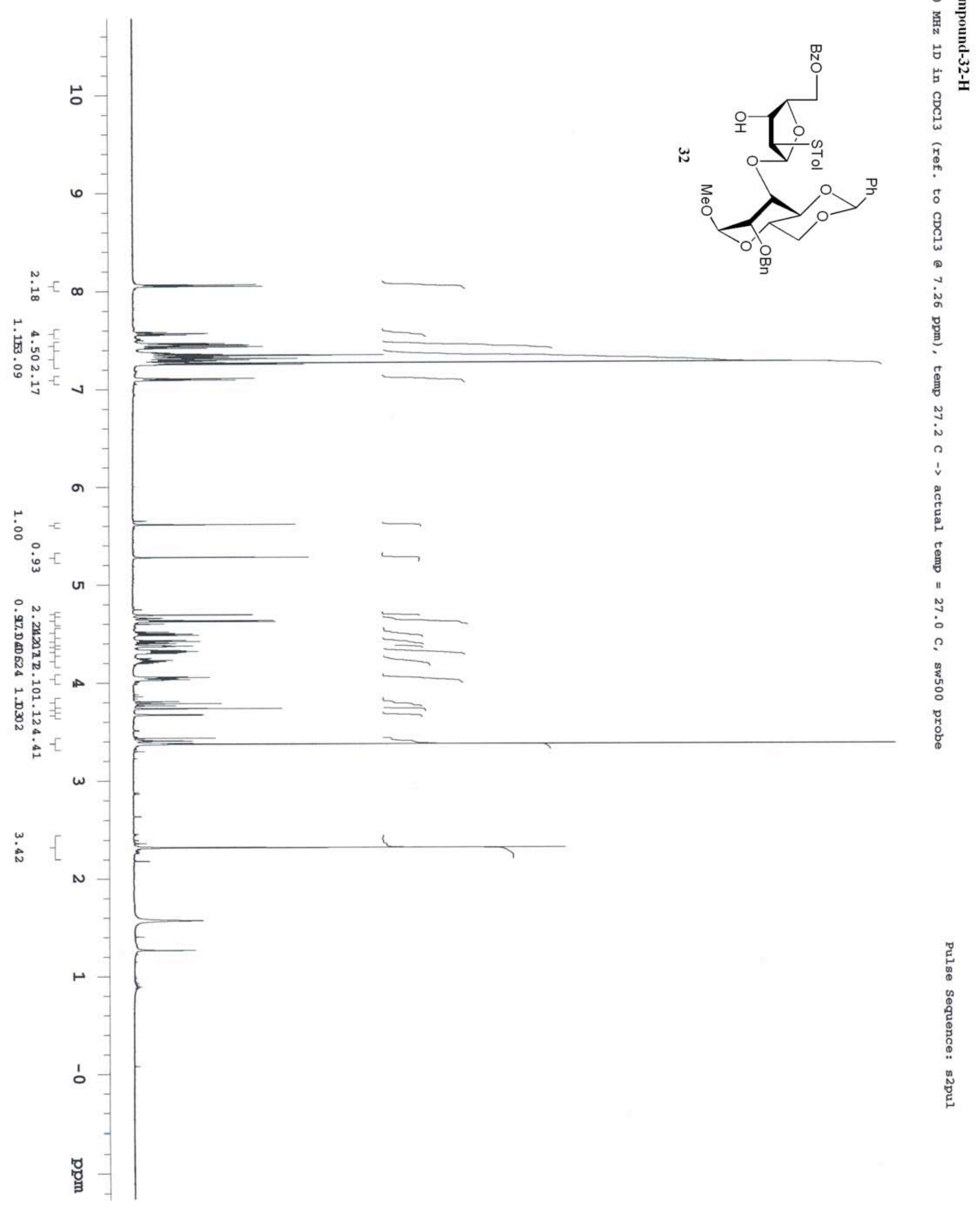



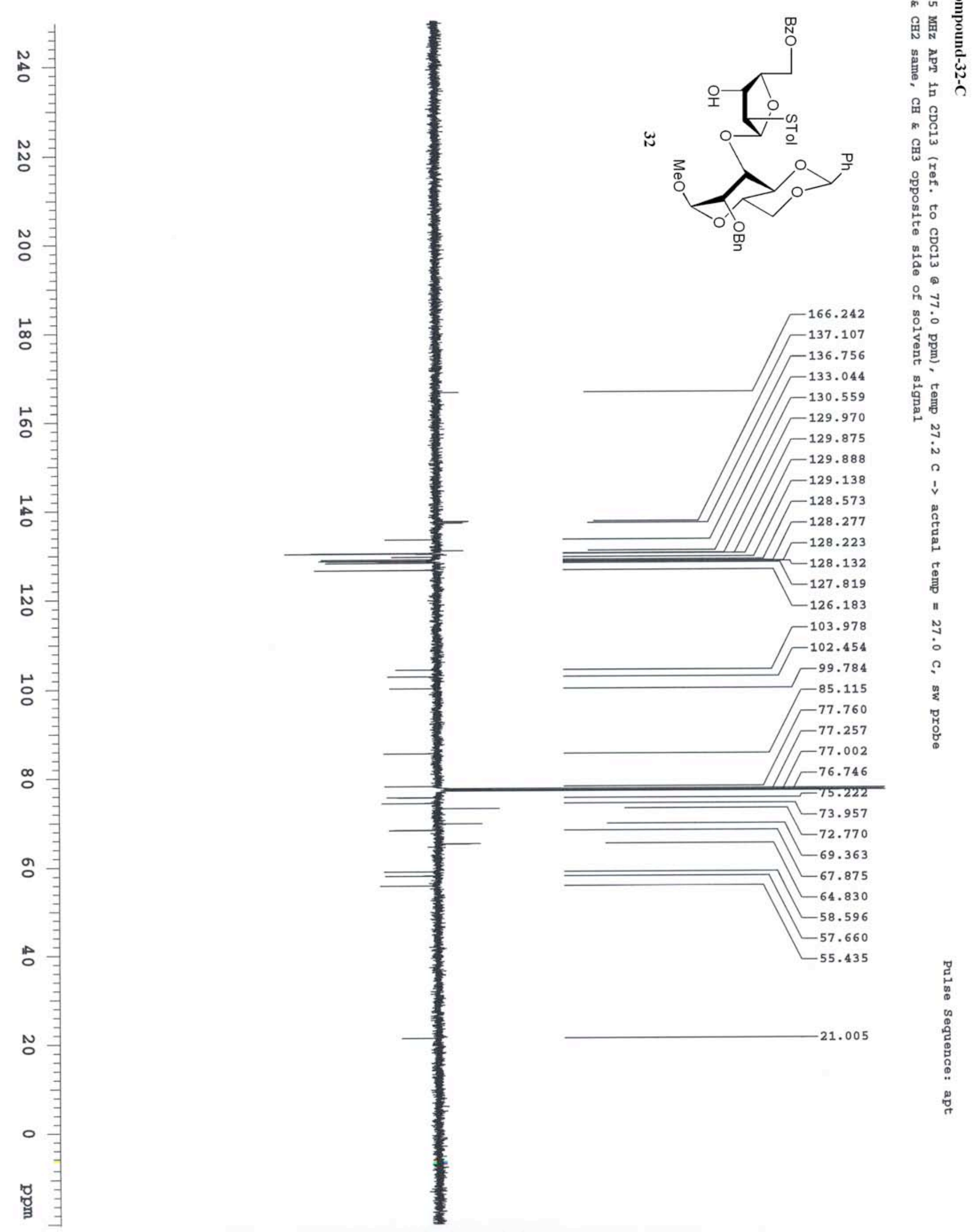


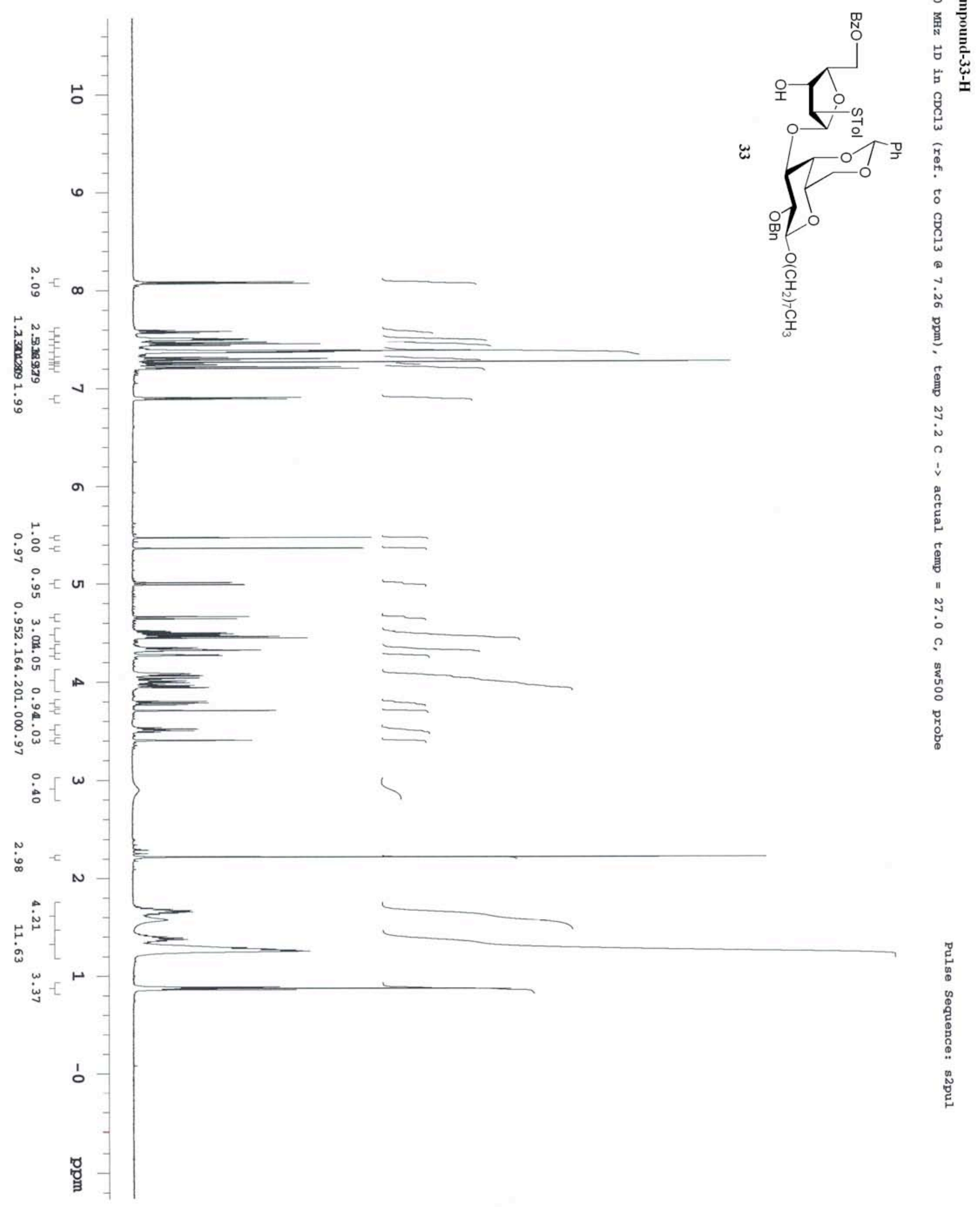




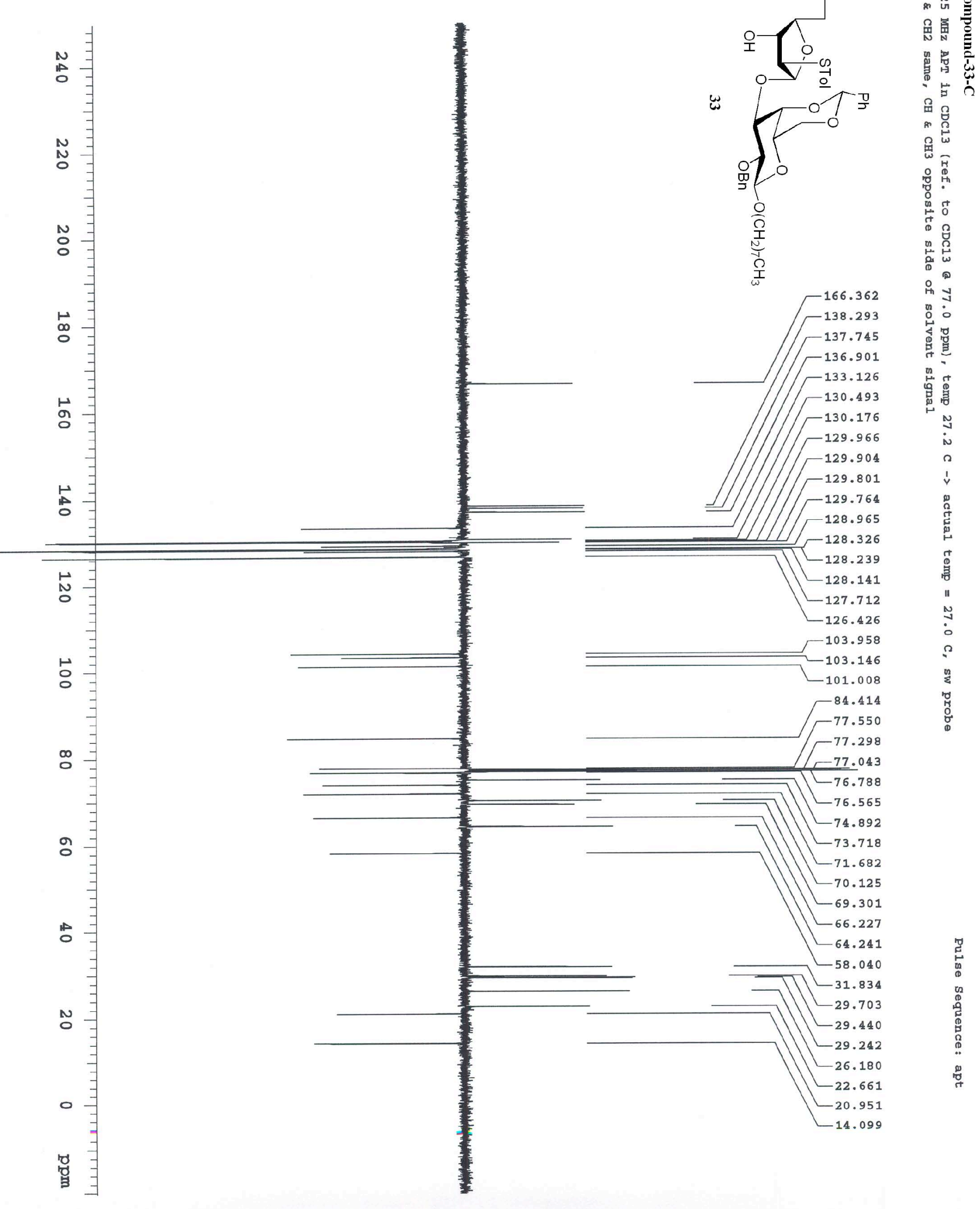



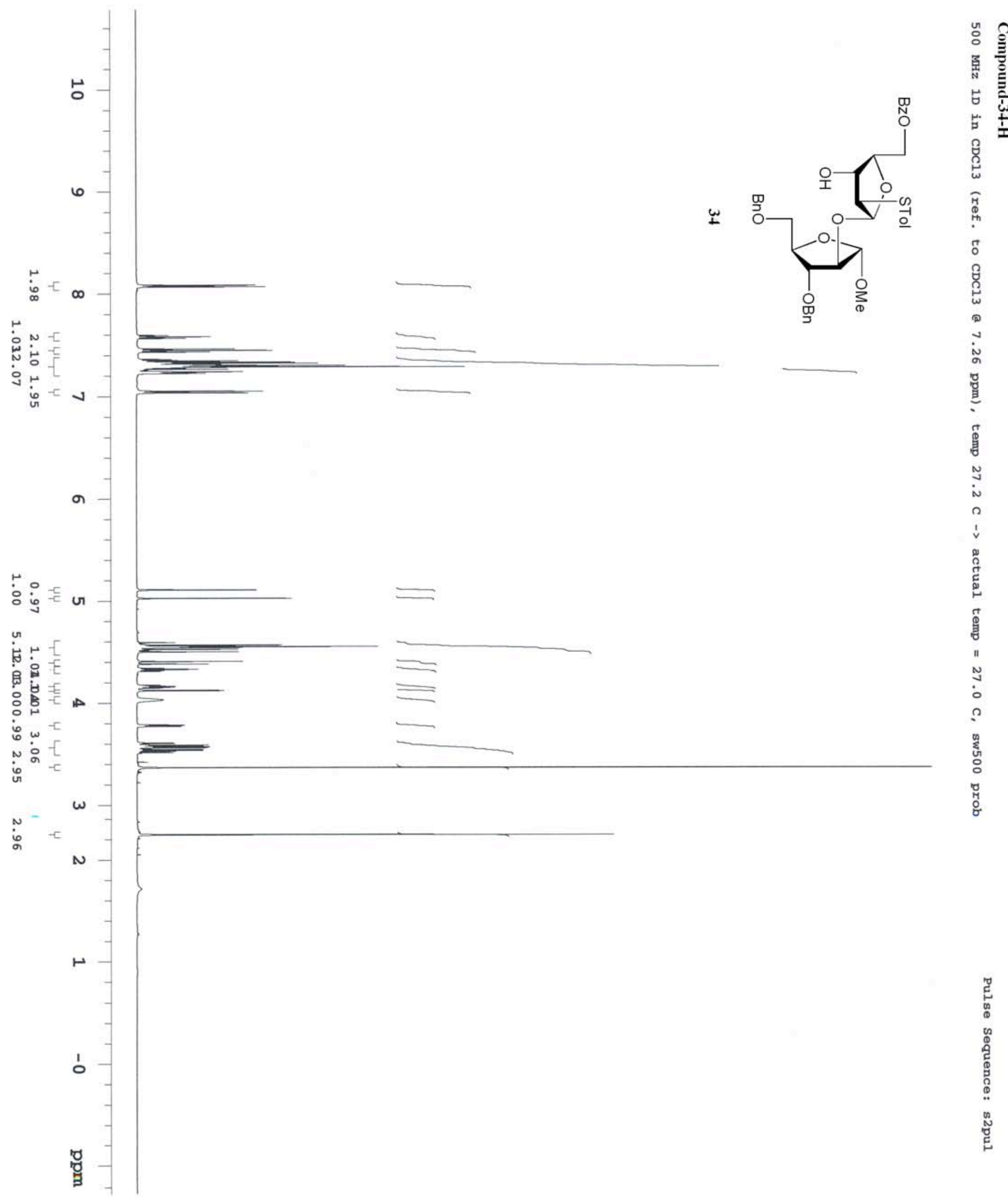


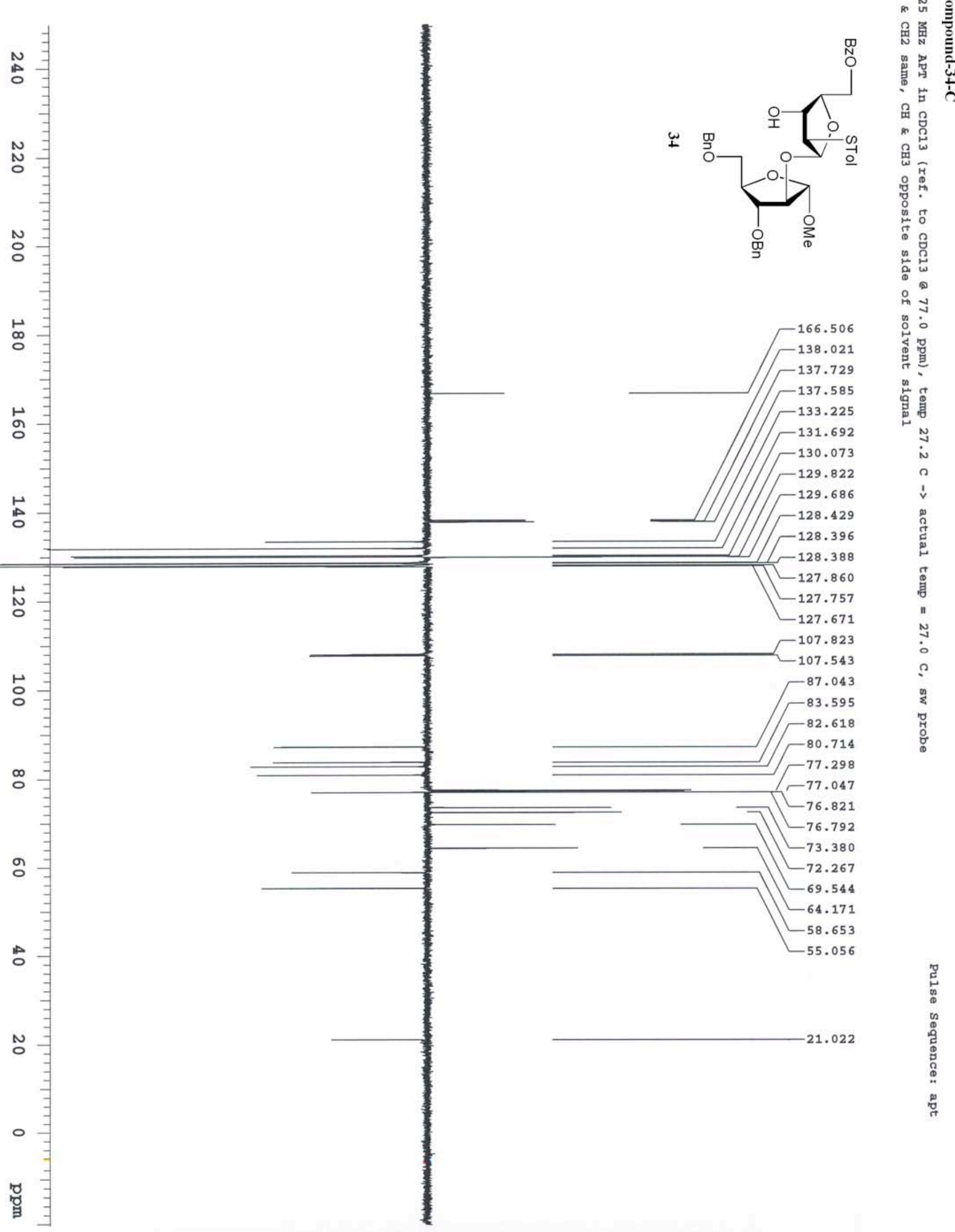



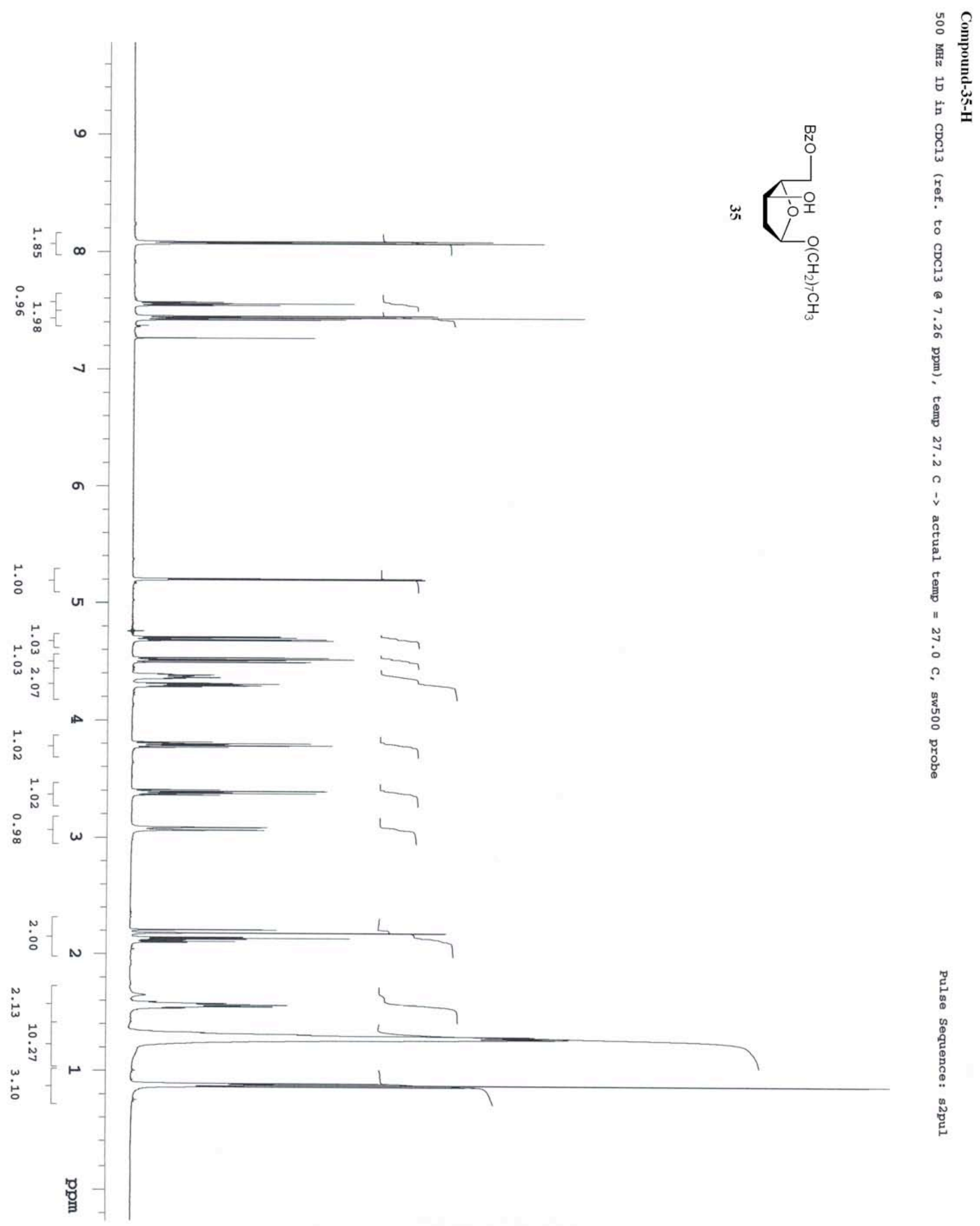


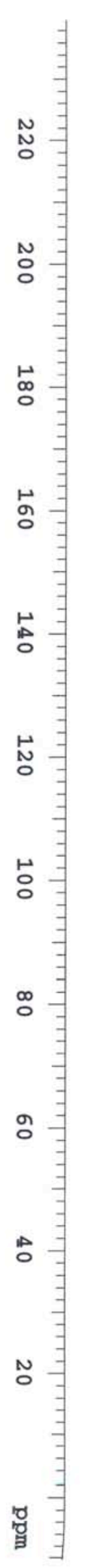

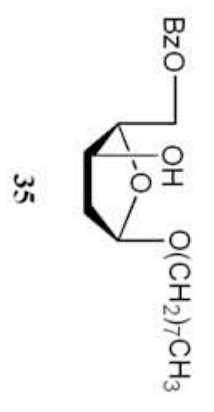

岕岕

掘兽

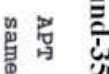

范

용ํำ

总

毘

ํํำ

孚

क०

品总

จ

in

安

$-166.436$

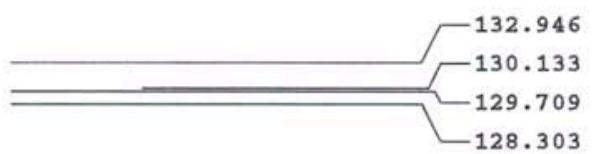

103.986

82.117

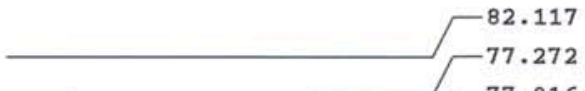

$-77.016$

$-71.667$

$-67.935$

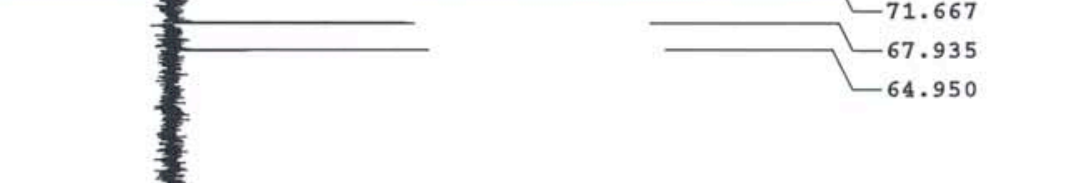

41.422

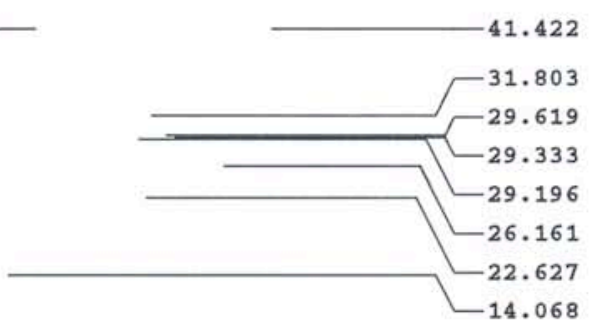

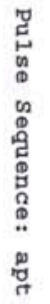




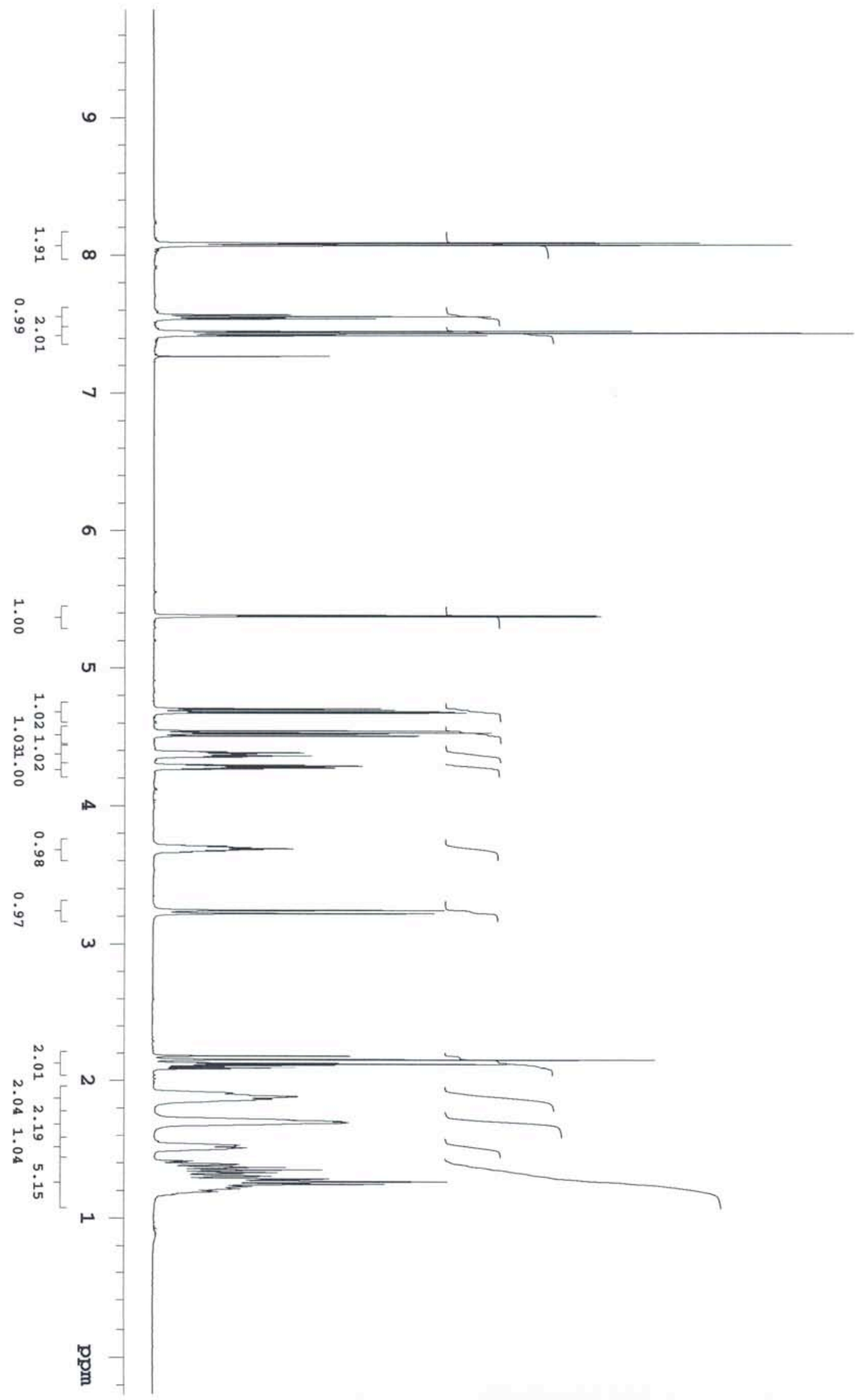




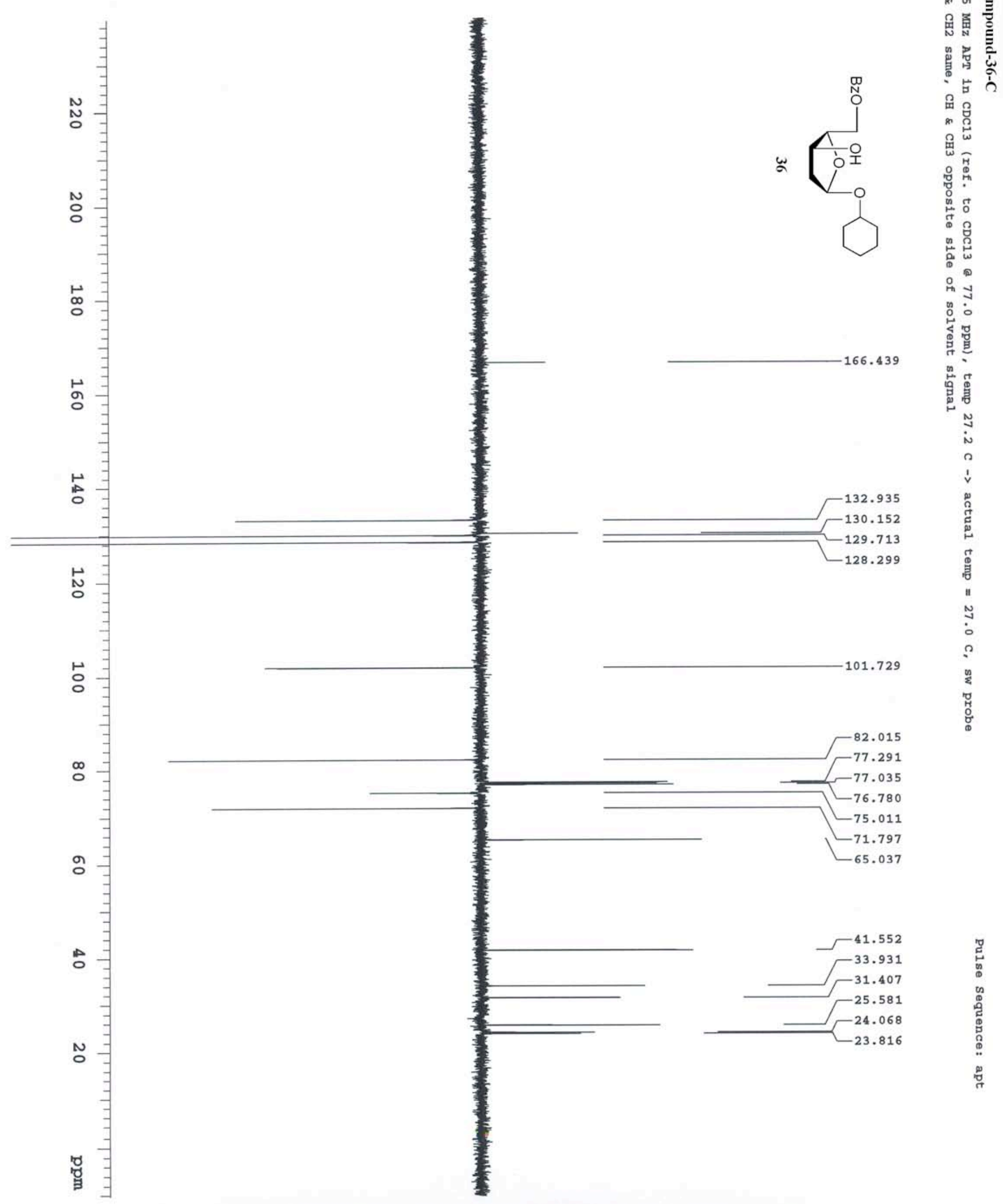




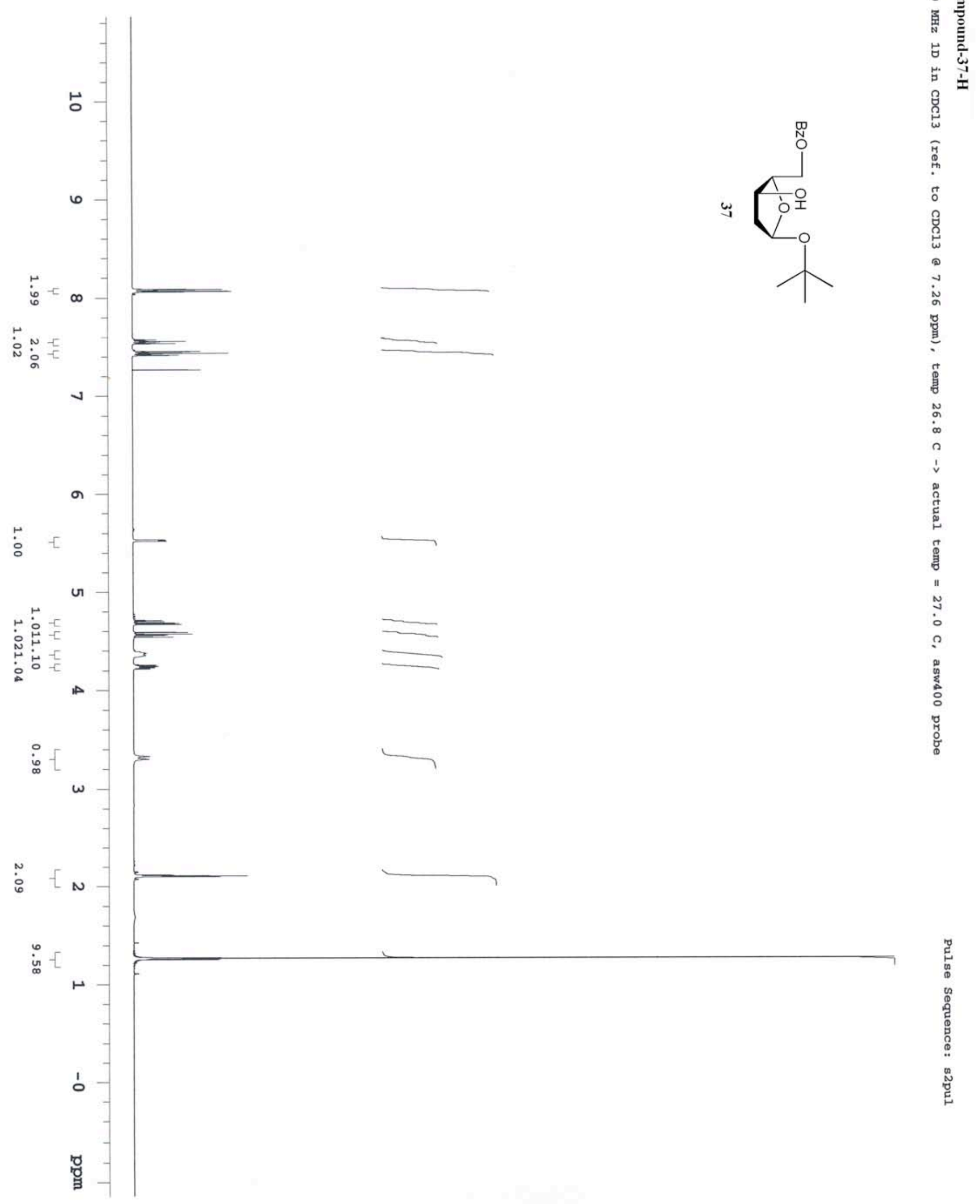




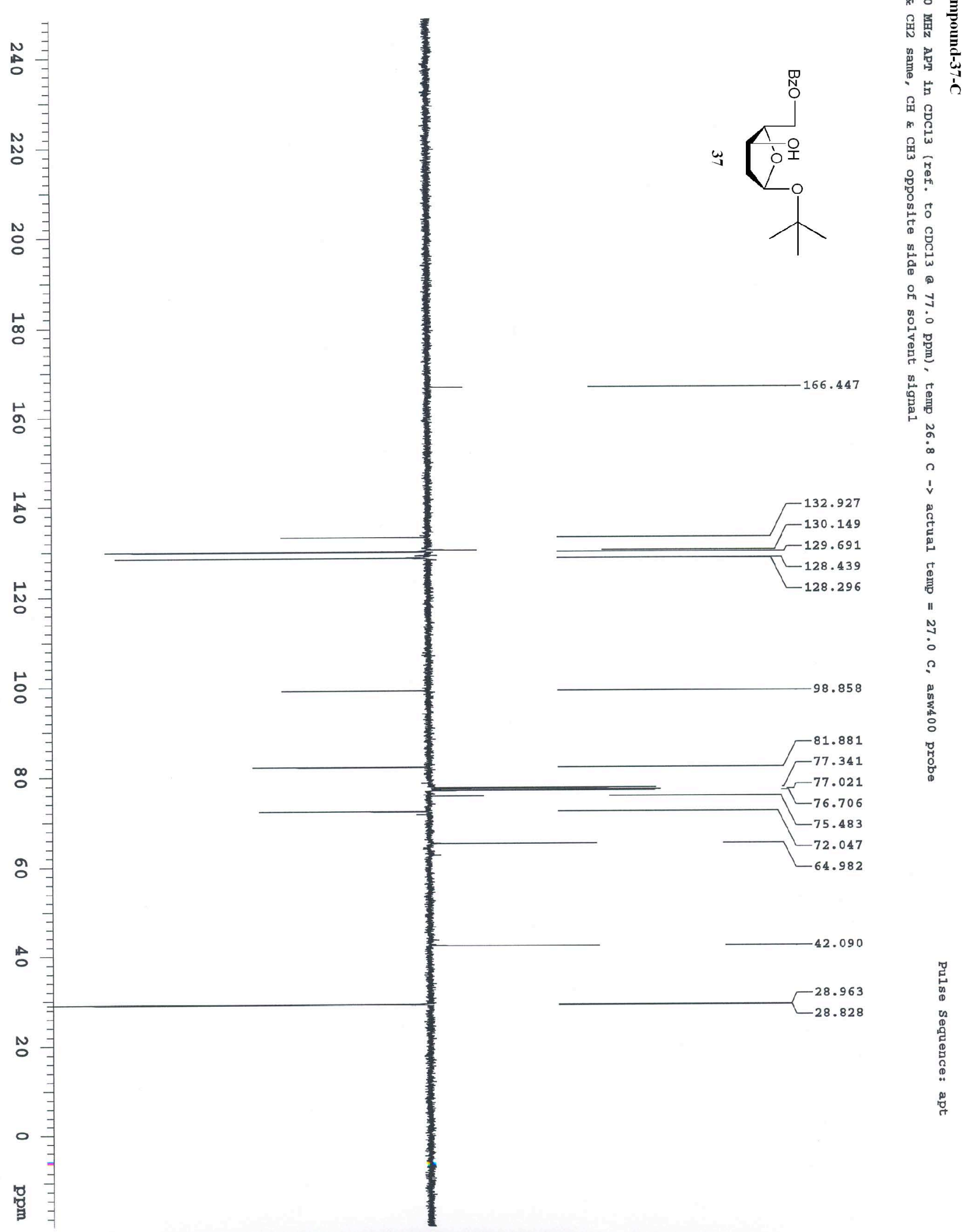




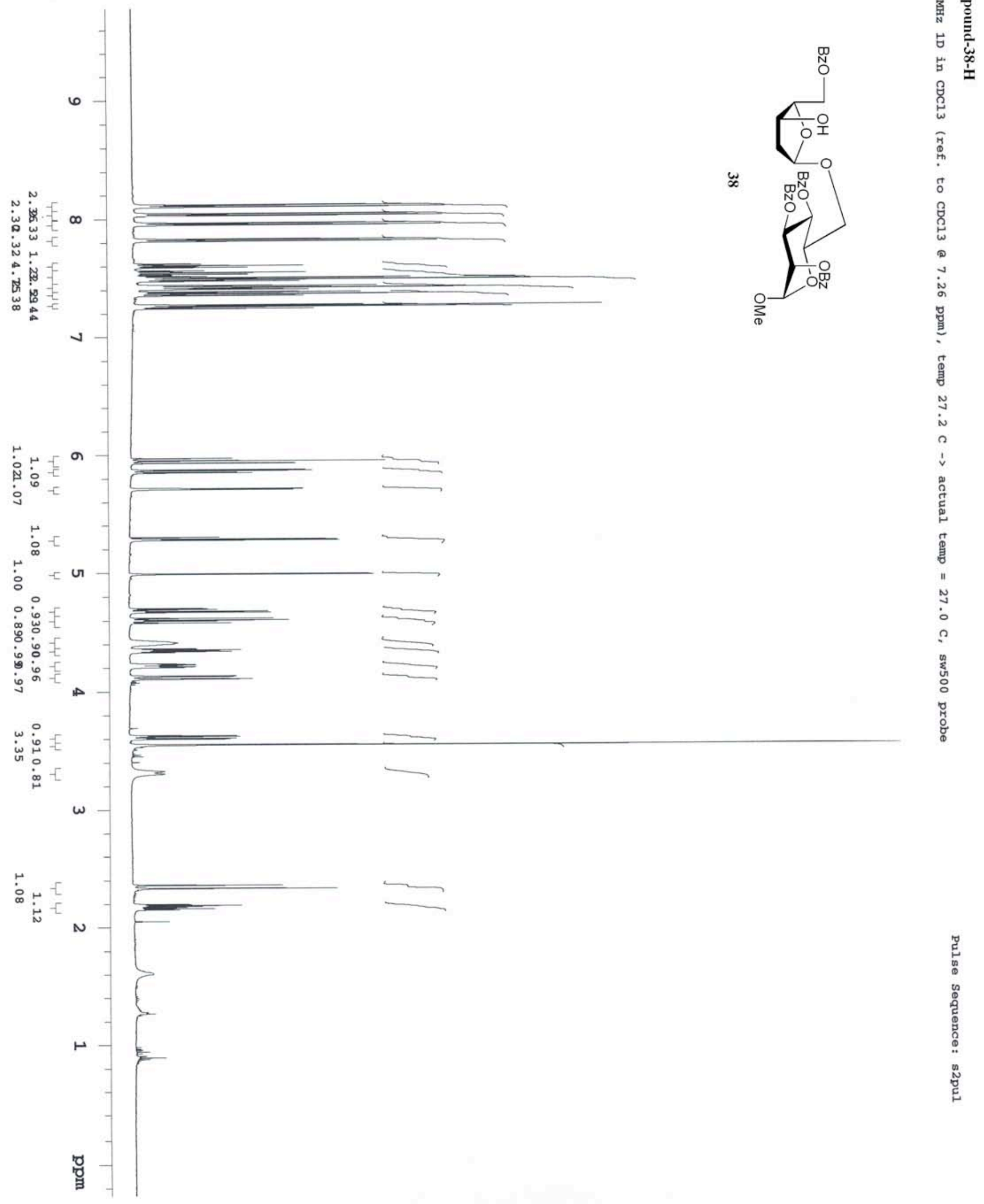




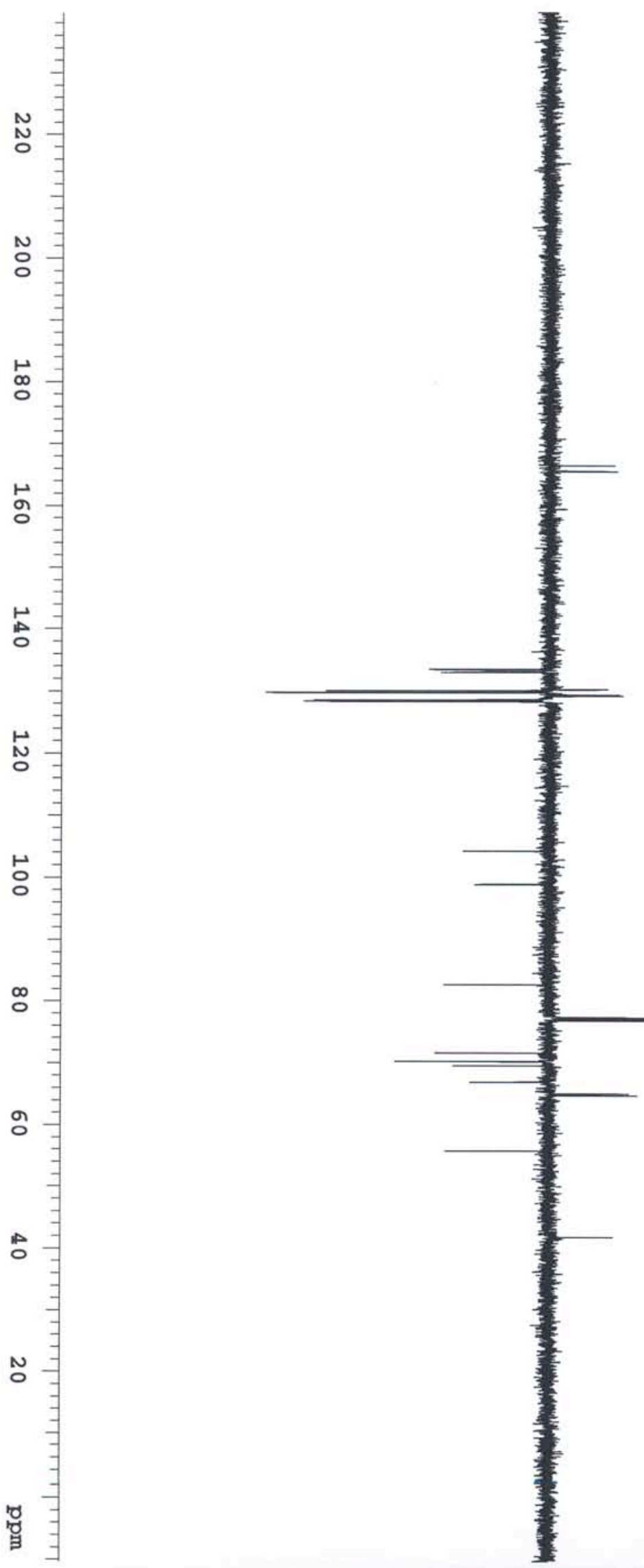

年

递

$\ddot{\infty}$

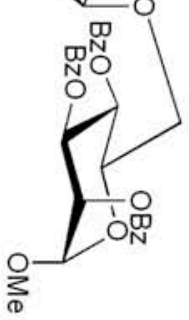

通

官

용

क्ष

i。

क्ष

के

雚总

ㅇำ

क

40

-166.359

$-165.509$

$-165.444$

是

总惫

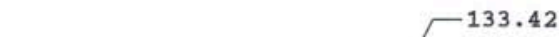

$-133.388$

$-132.916$

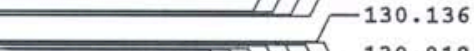

$-129.74$

$-129.728$

$-129.686$

$-129.332$

$-129.214$

$-129.088$

$-128.535$

$-98.813$

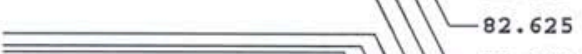

$-77.268$

$-77.013$

$-76.757$

$-71.515$

$-70.158$

$-69.471$

$-66.864$

$-64.934$

$-64.660$

$-55.632$

$-41.666$ 


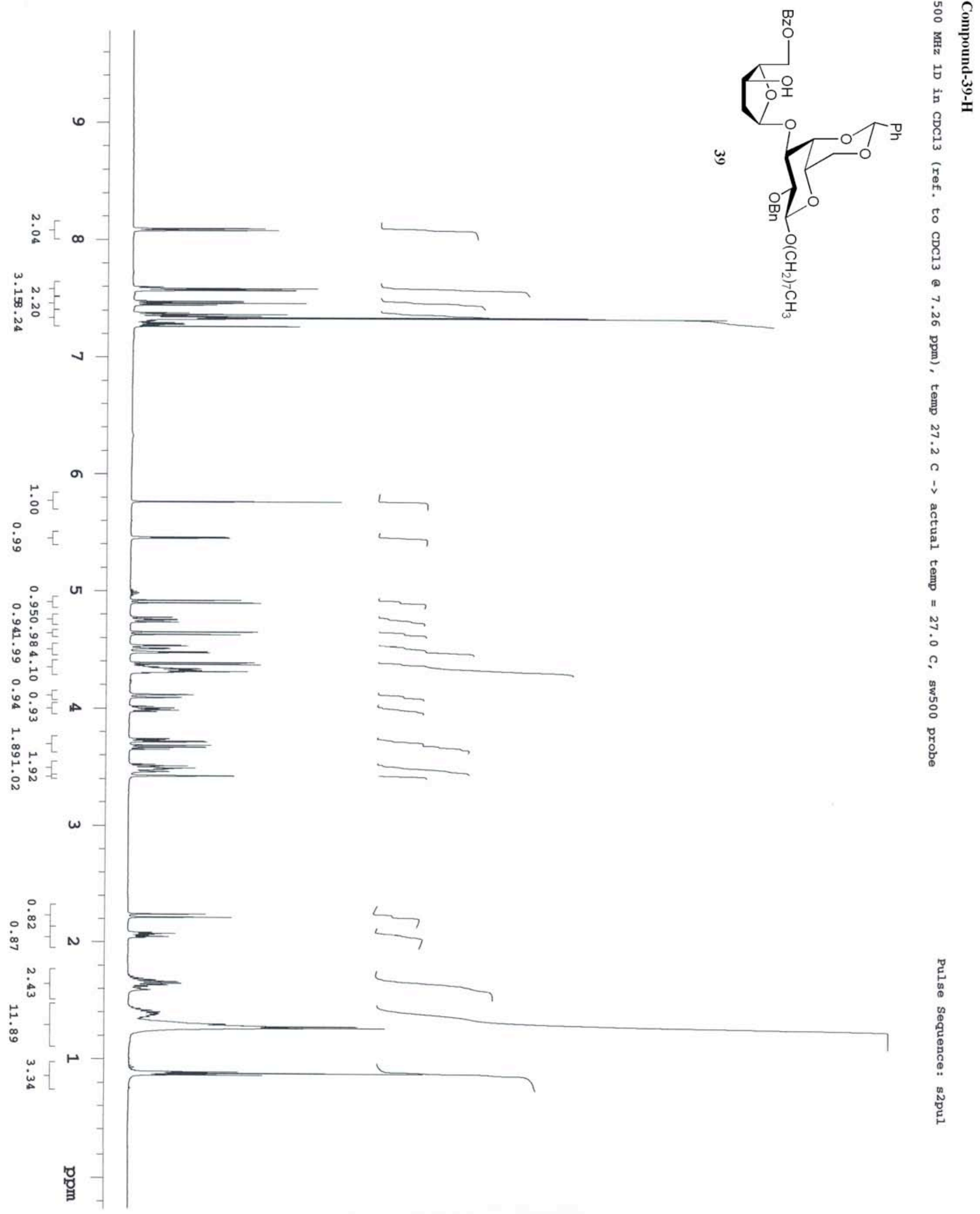



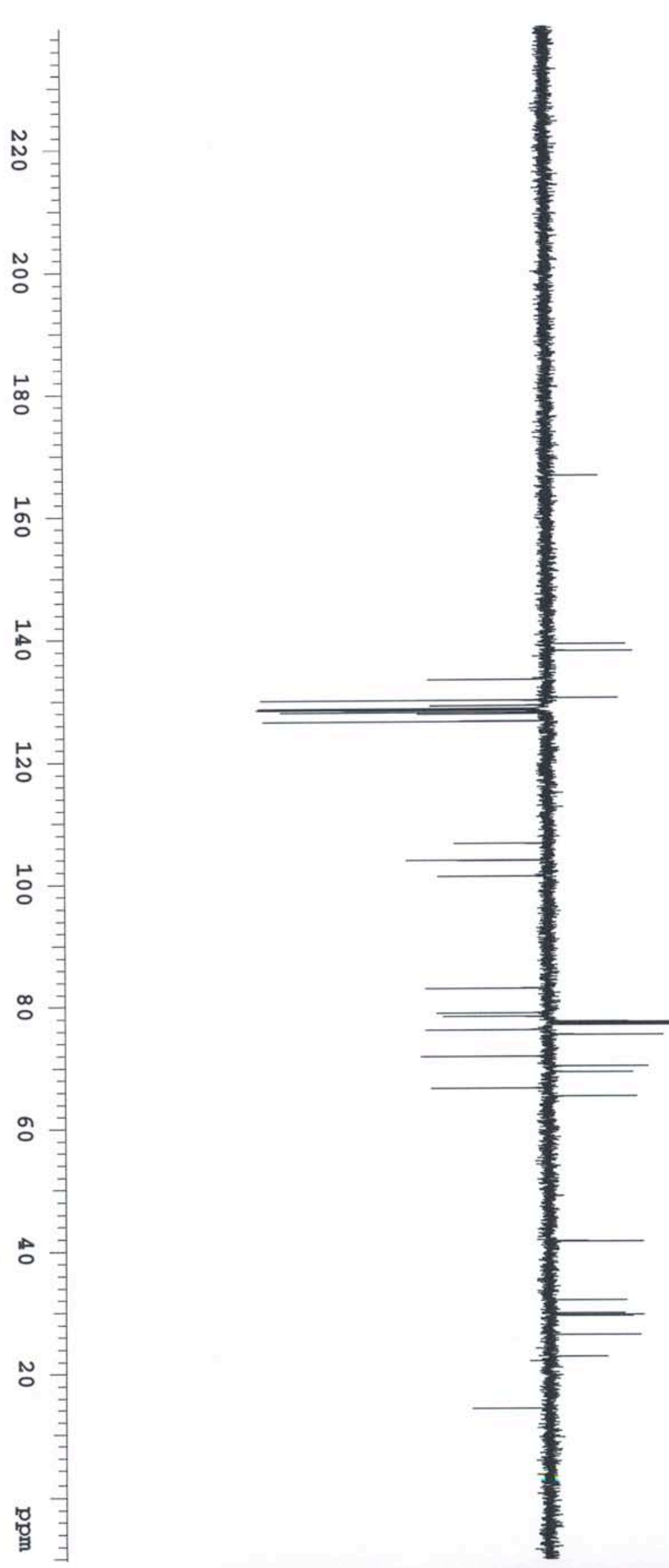

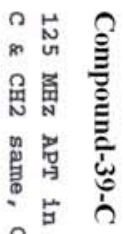
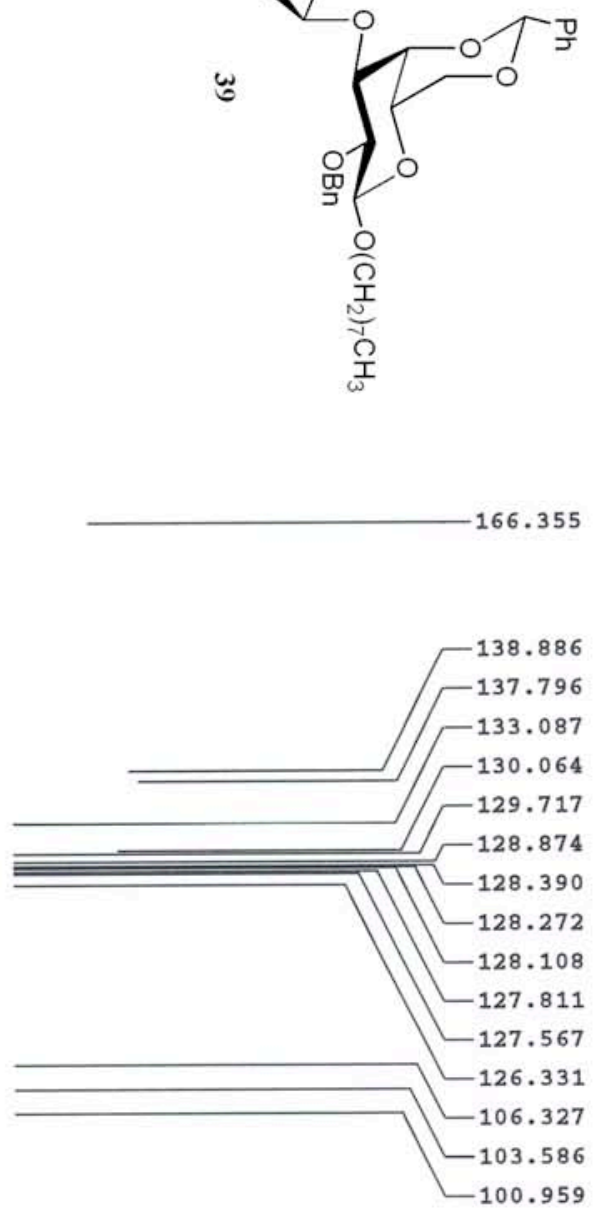

午

i

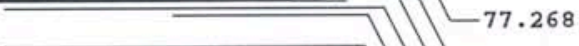

$-77.013$

$-76.757$

$-75.941$

$-75.152$

$-71.583$

$-70.001$

$-69.052$

$-66.398$

$-65.045$

$-41.365$

$-31.830$

$-29.695$

$-29.440$

$-29.268$

$-26.157$

$-22.661$

$-14.087$ 


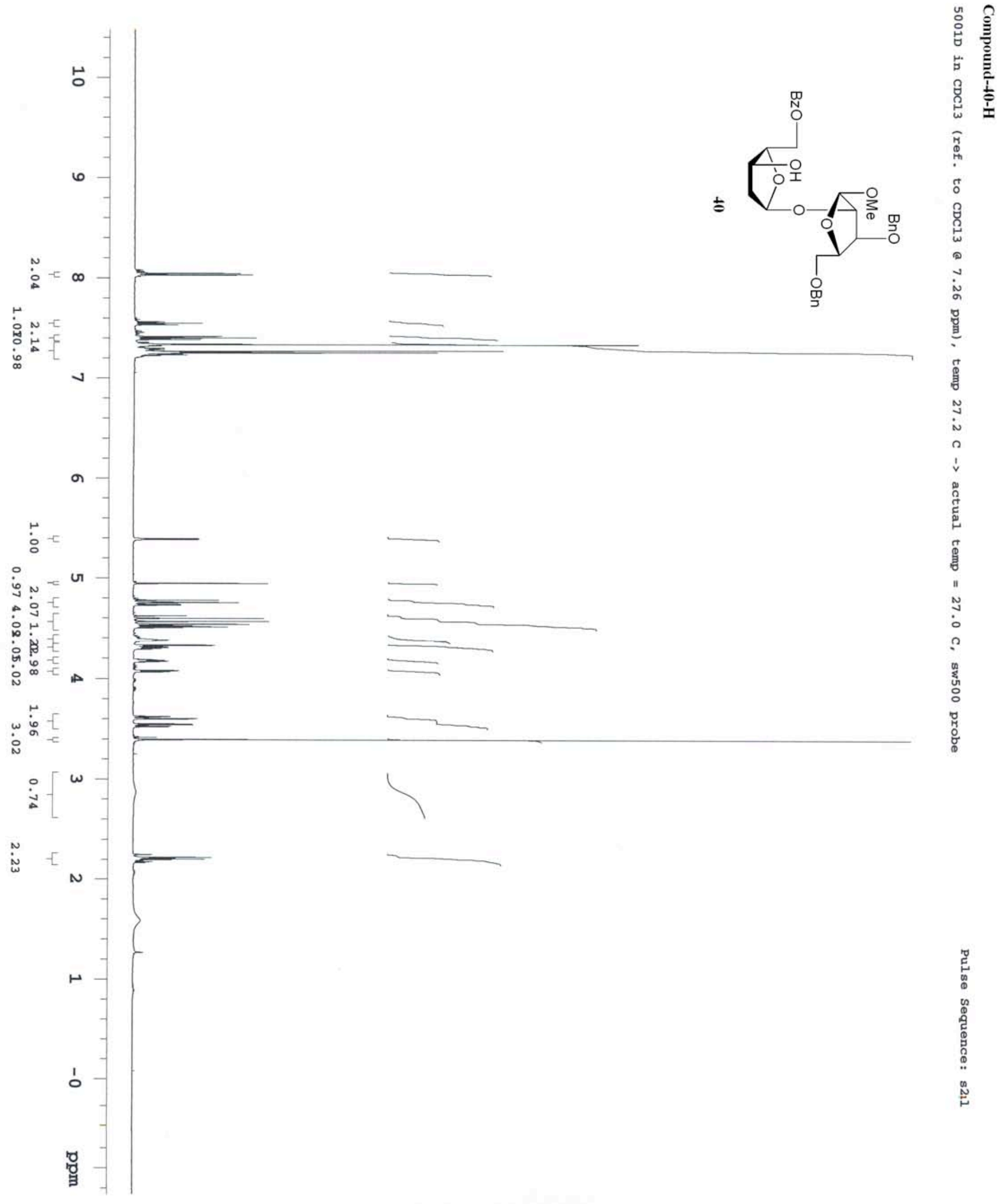



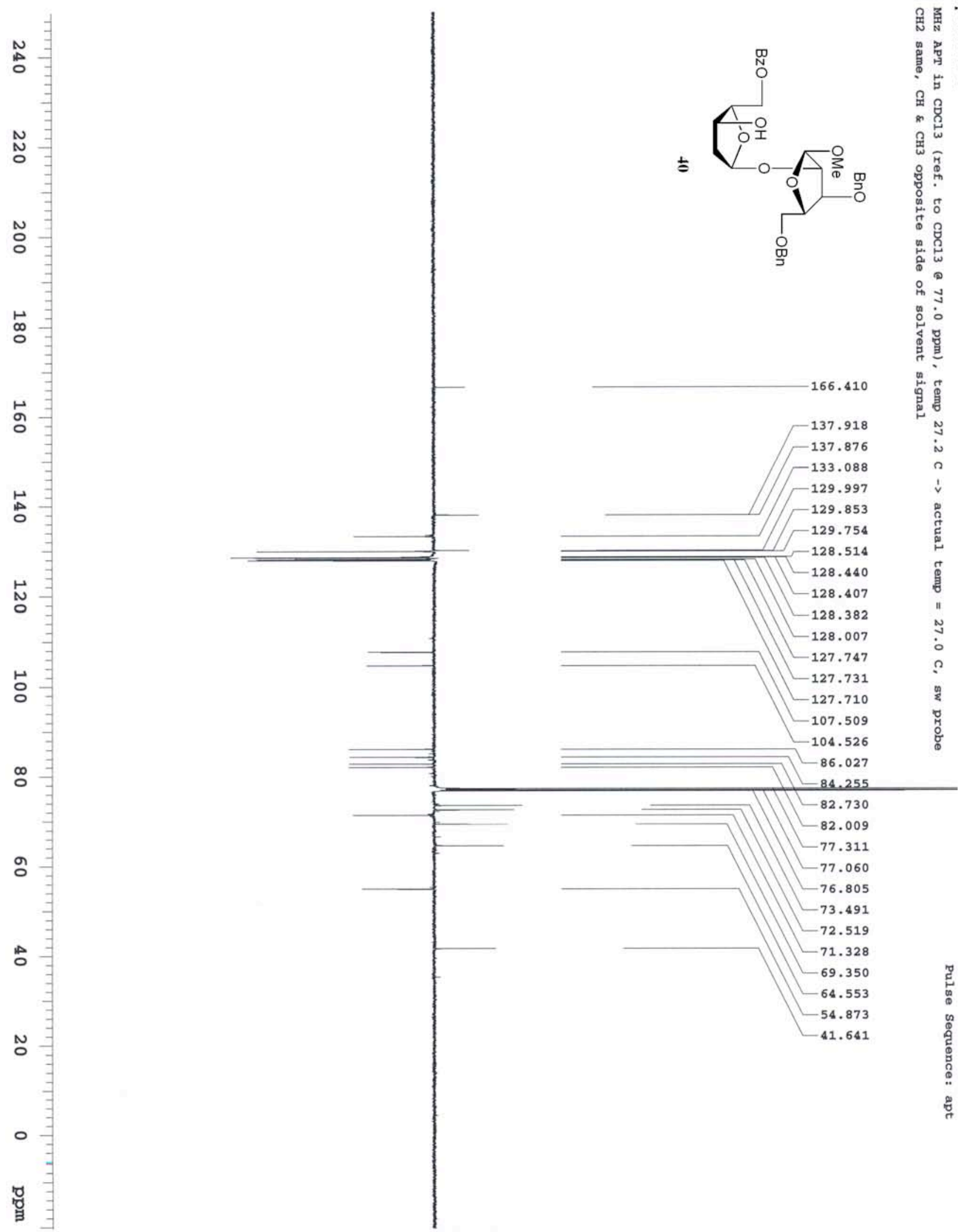


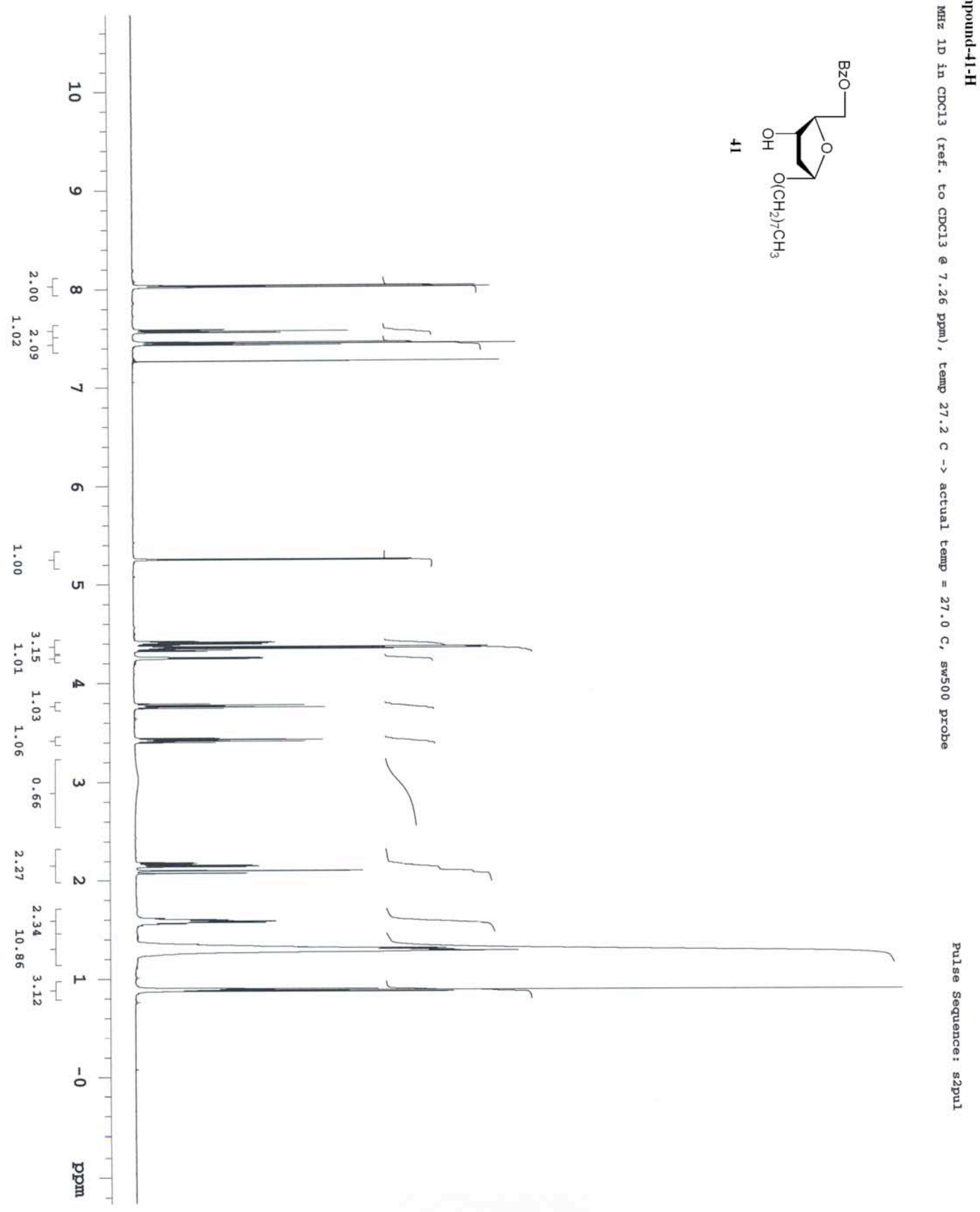



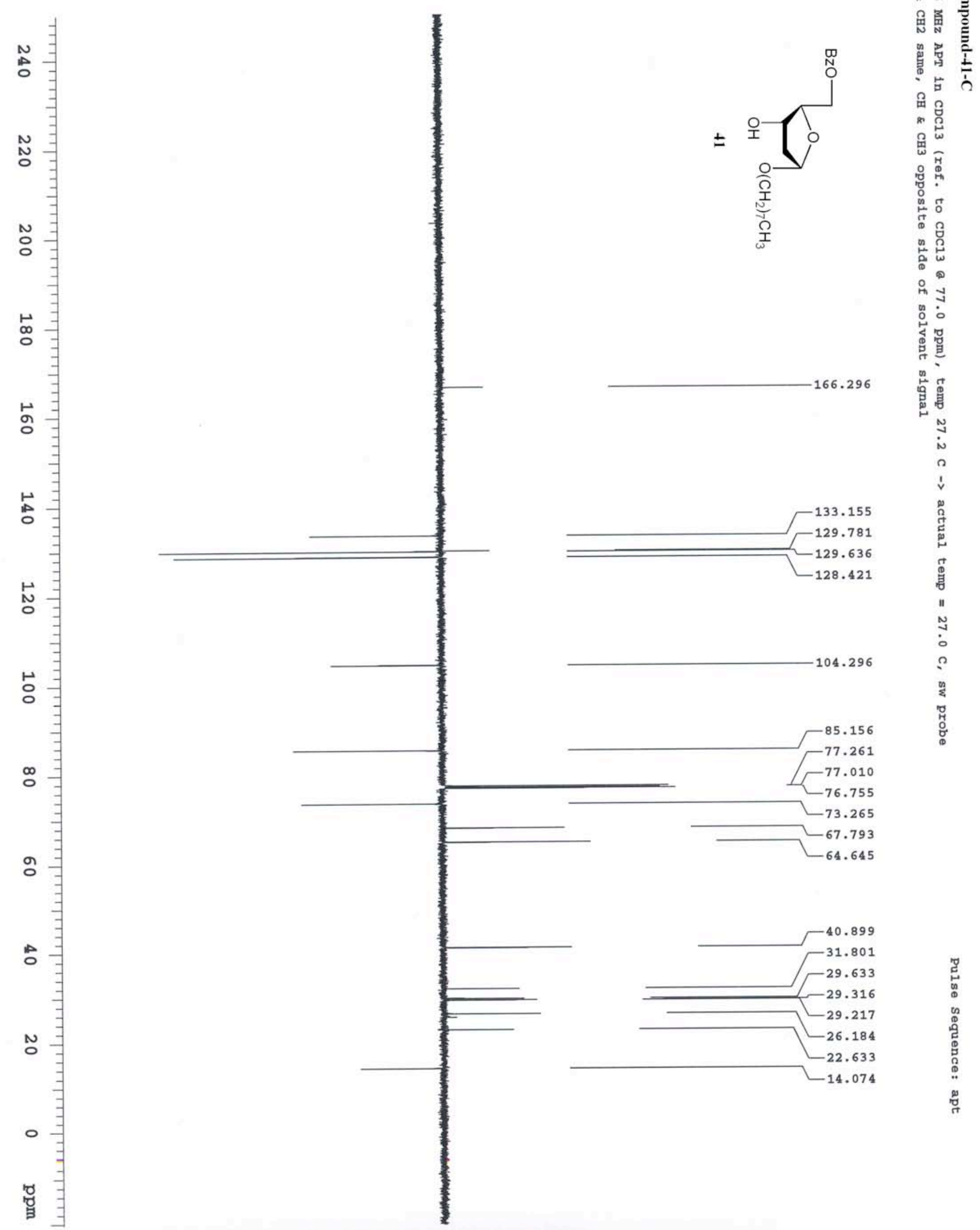


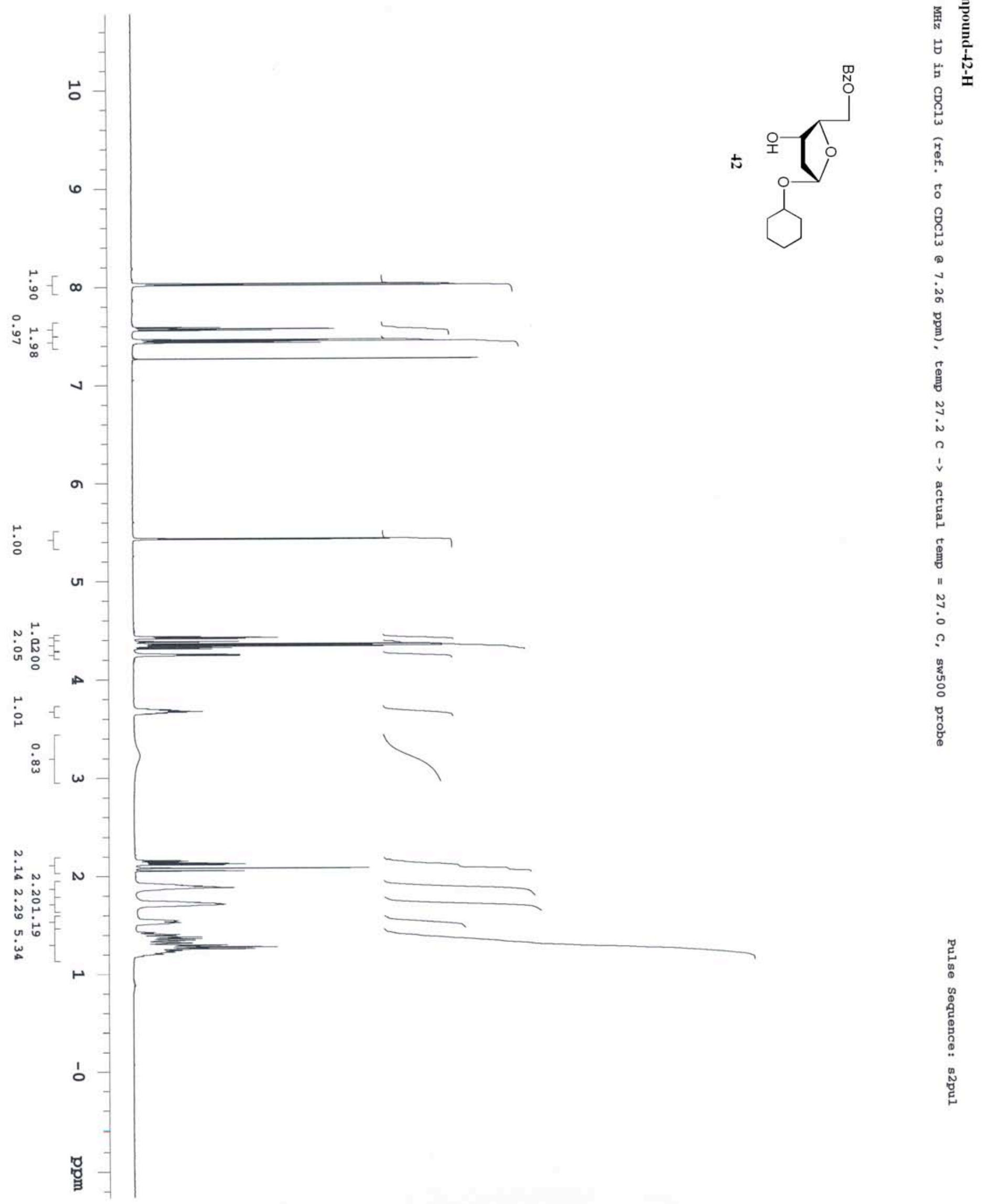



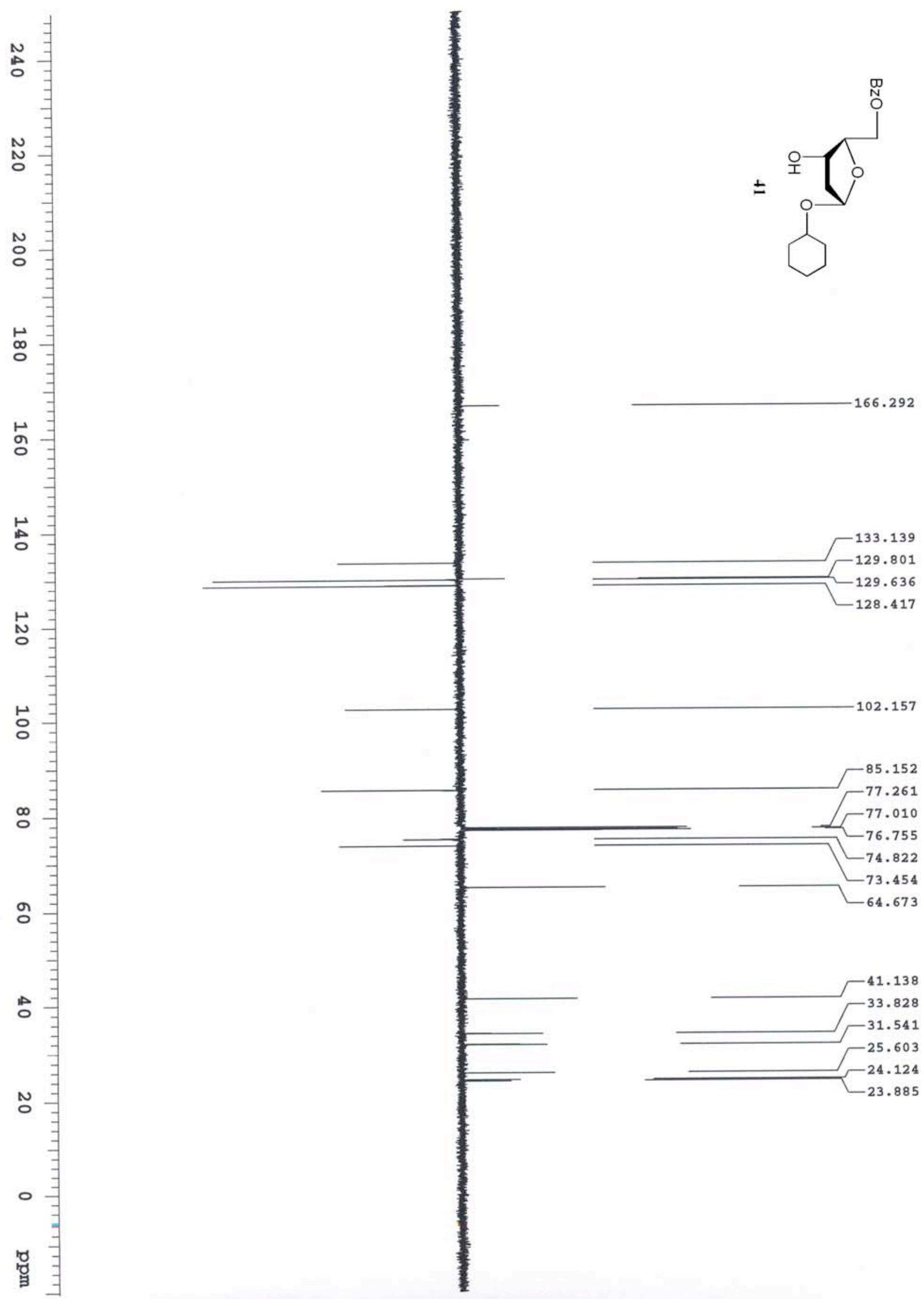

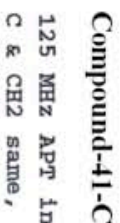

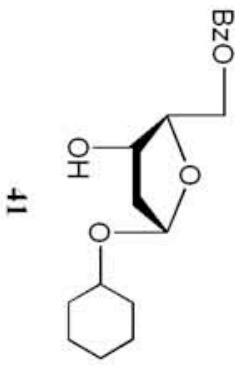

鱼

,

罟

용

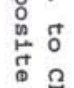

㤎总

क

os:

畤

$-166.292$

总点

i

\&

愬

兽

:

$-102.157$

骂

'

$-77.261$

$-76.755$

$-74.822$

$-73.454$

$-64.673$

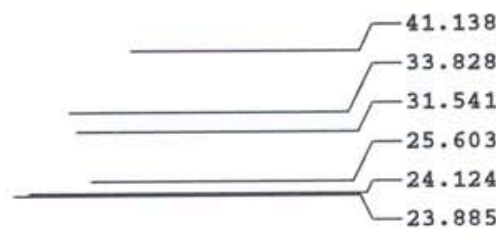



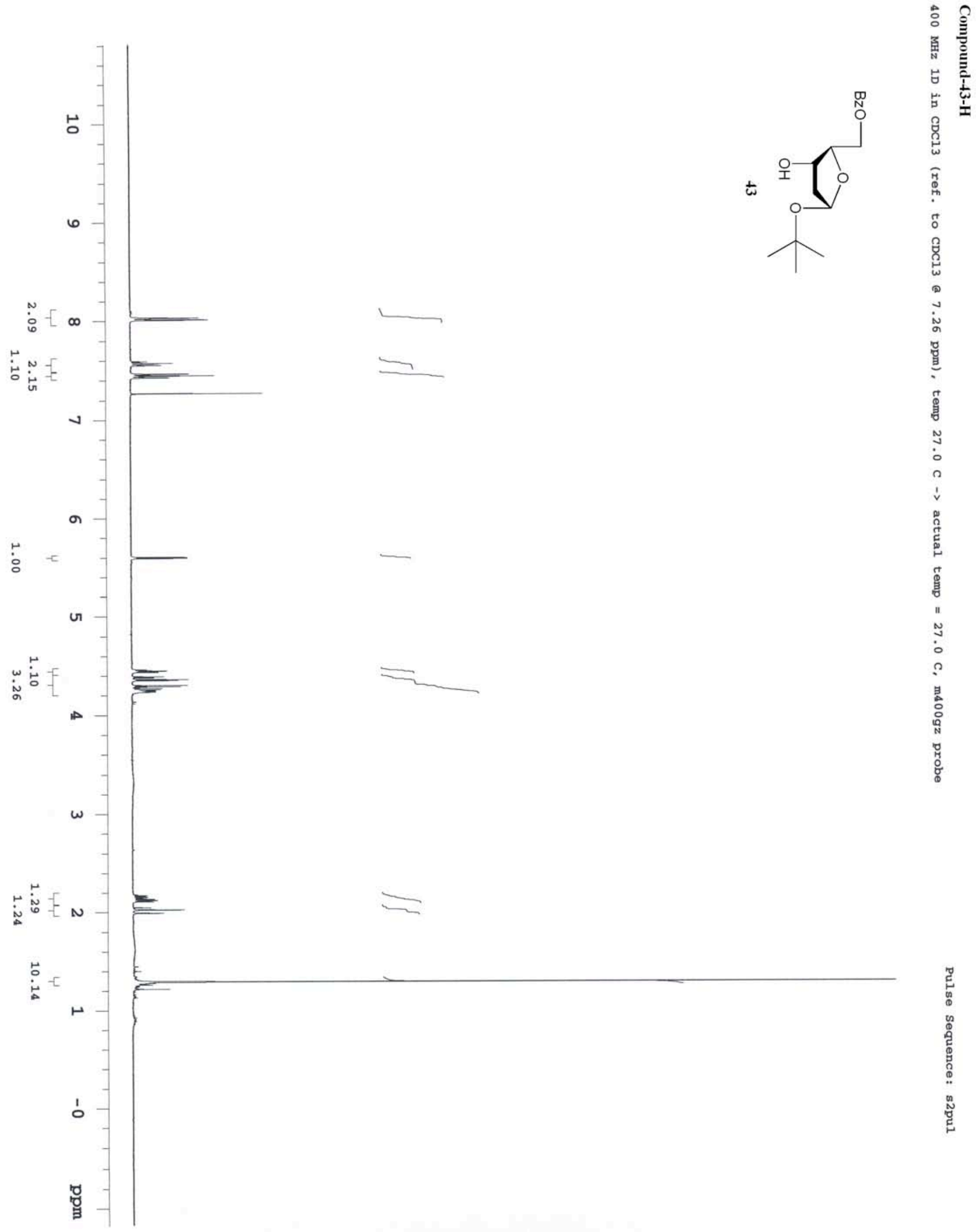


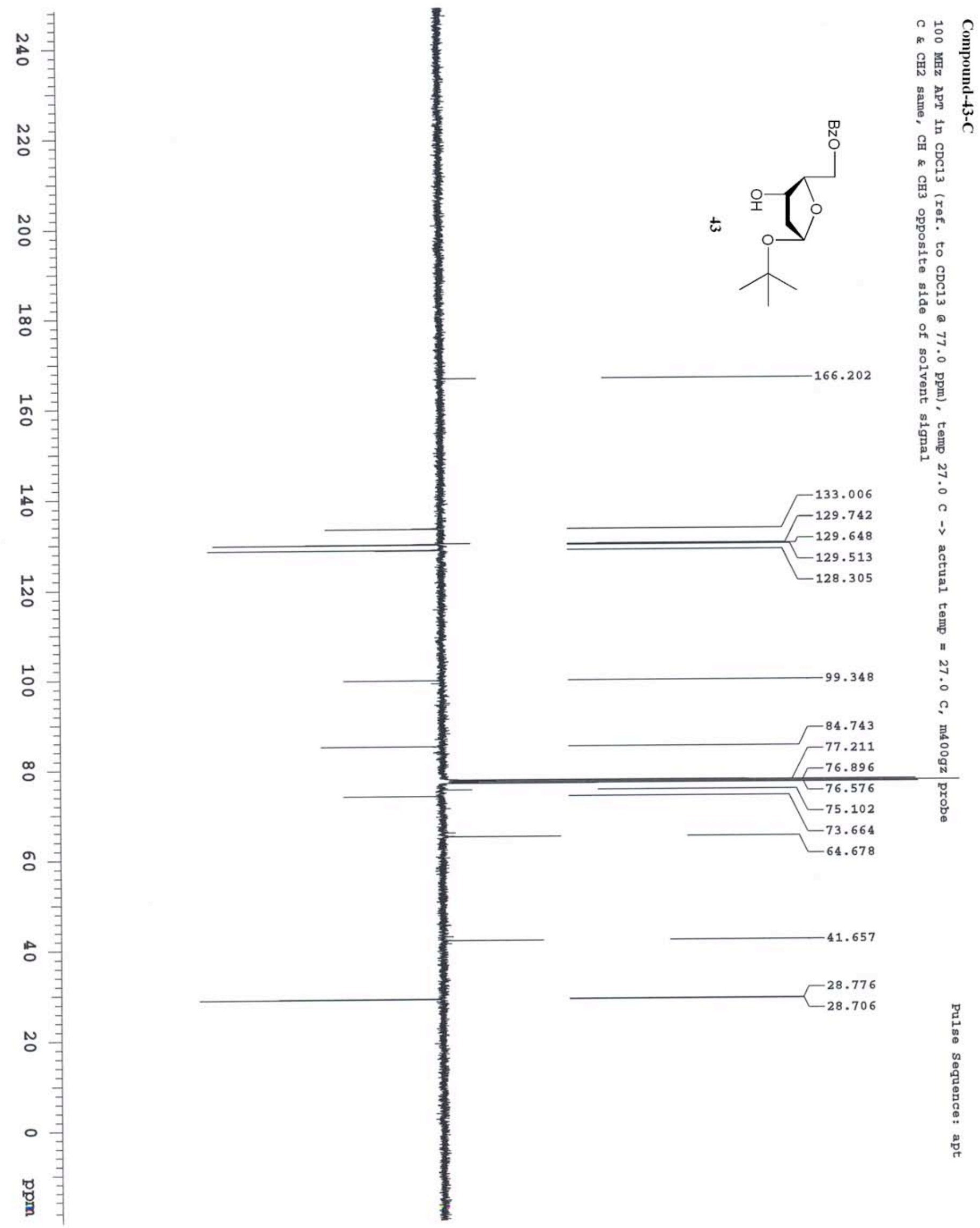




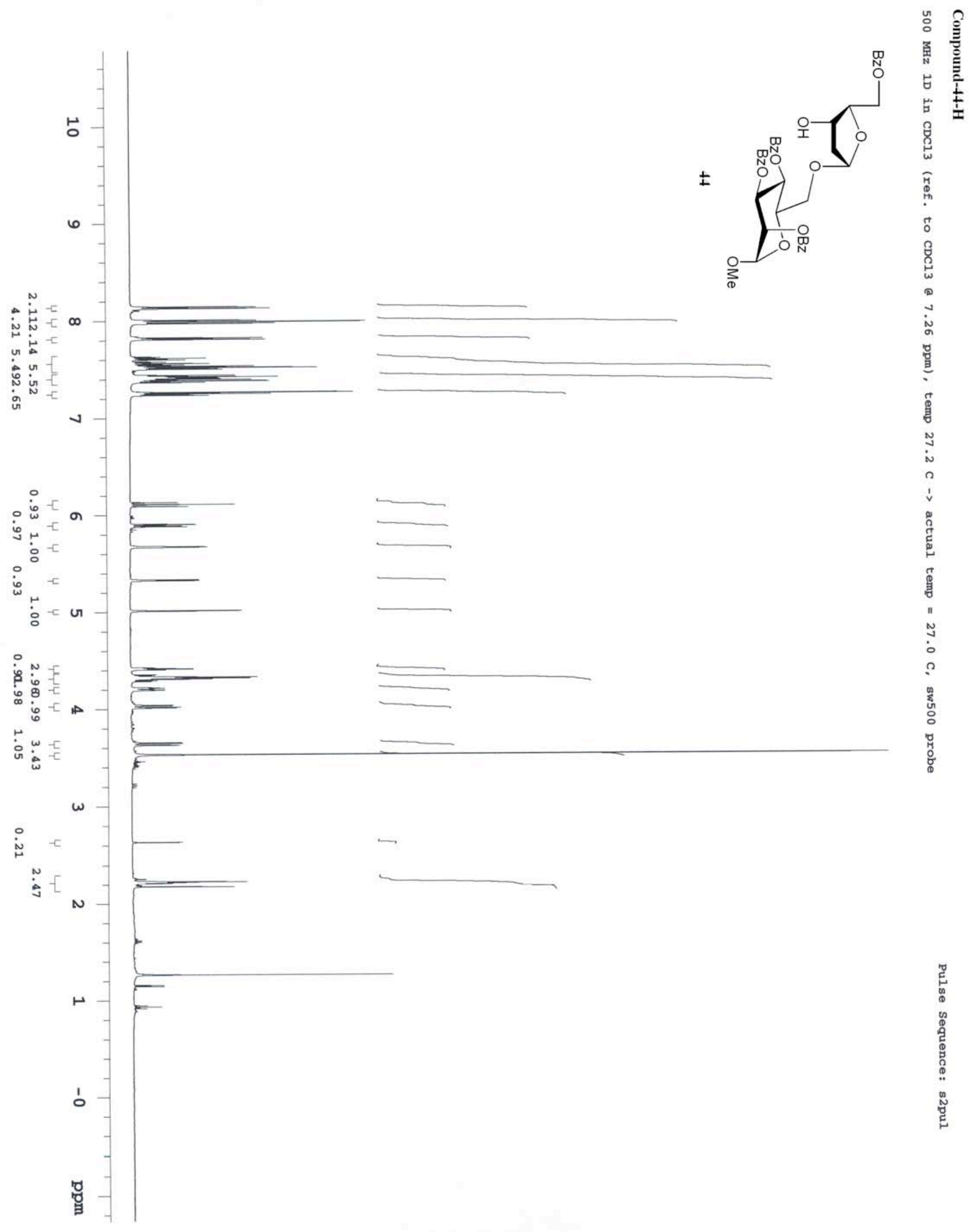




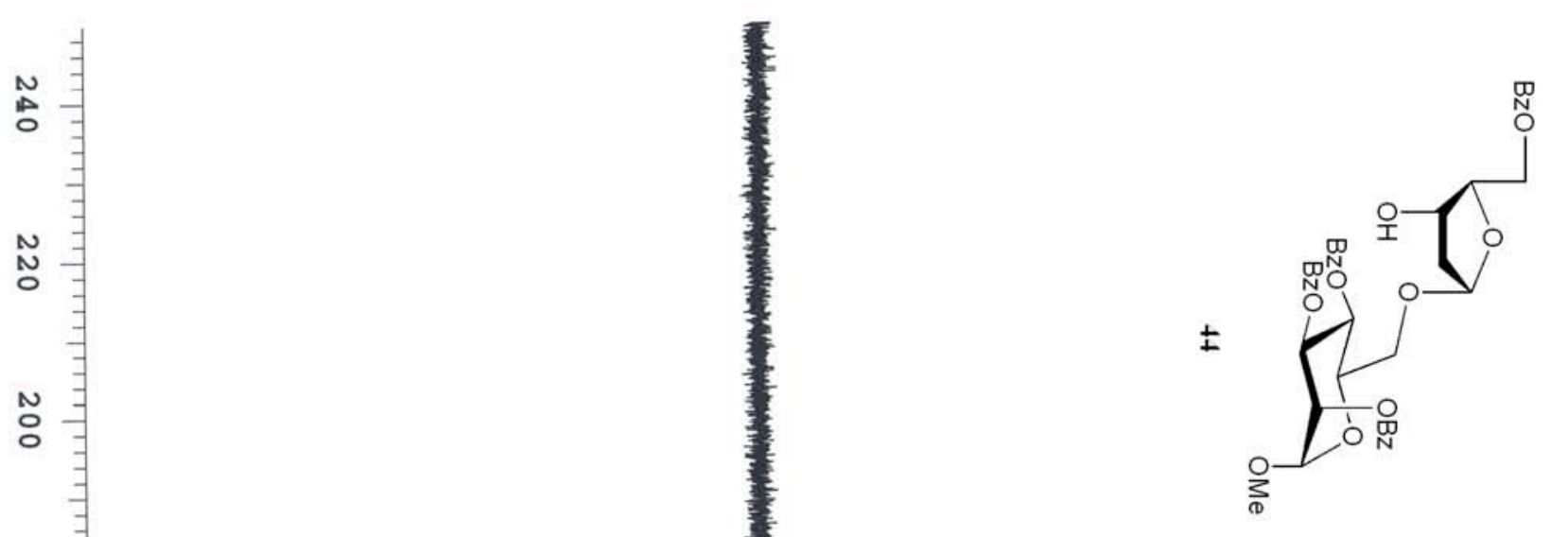

语

$\stackrel{+}{\infty}$

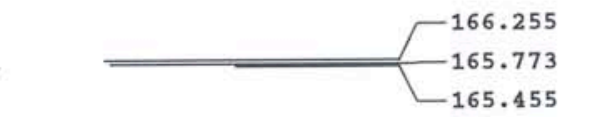

ํㅗㅇ

战

宫

岕

농

용-

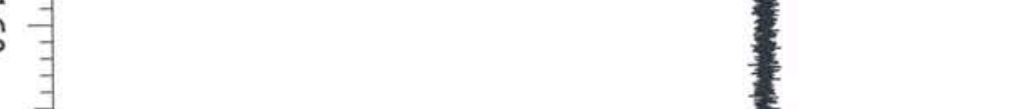

$\stackrel{\infty}{\circ}$

$\stackrel{\infty}{\circ}$

응

g)

응

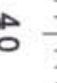

ก

${ }^{133.551}$

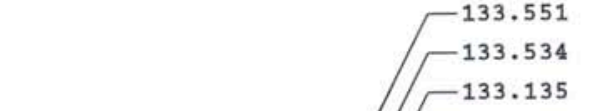

$\iint^{133.135}$

-129.764

$-129.719$

$-129.616$

$-129.344$

$-129.072$

$-128.956$

$-128.668$

$-128.511$

$-128.404$

$-128.272$

$-104.844$

$-98.733$

$-77.278$

$-77.027$

$-73.124$

$-70.417$

$-69.906$

$-69.453$

$-66.717$

$-65.374$

$-64.744$

$-55.641$

$-41.636$ 


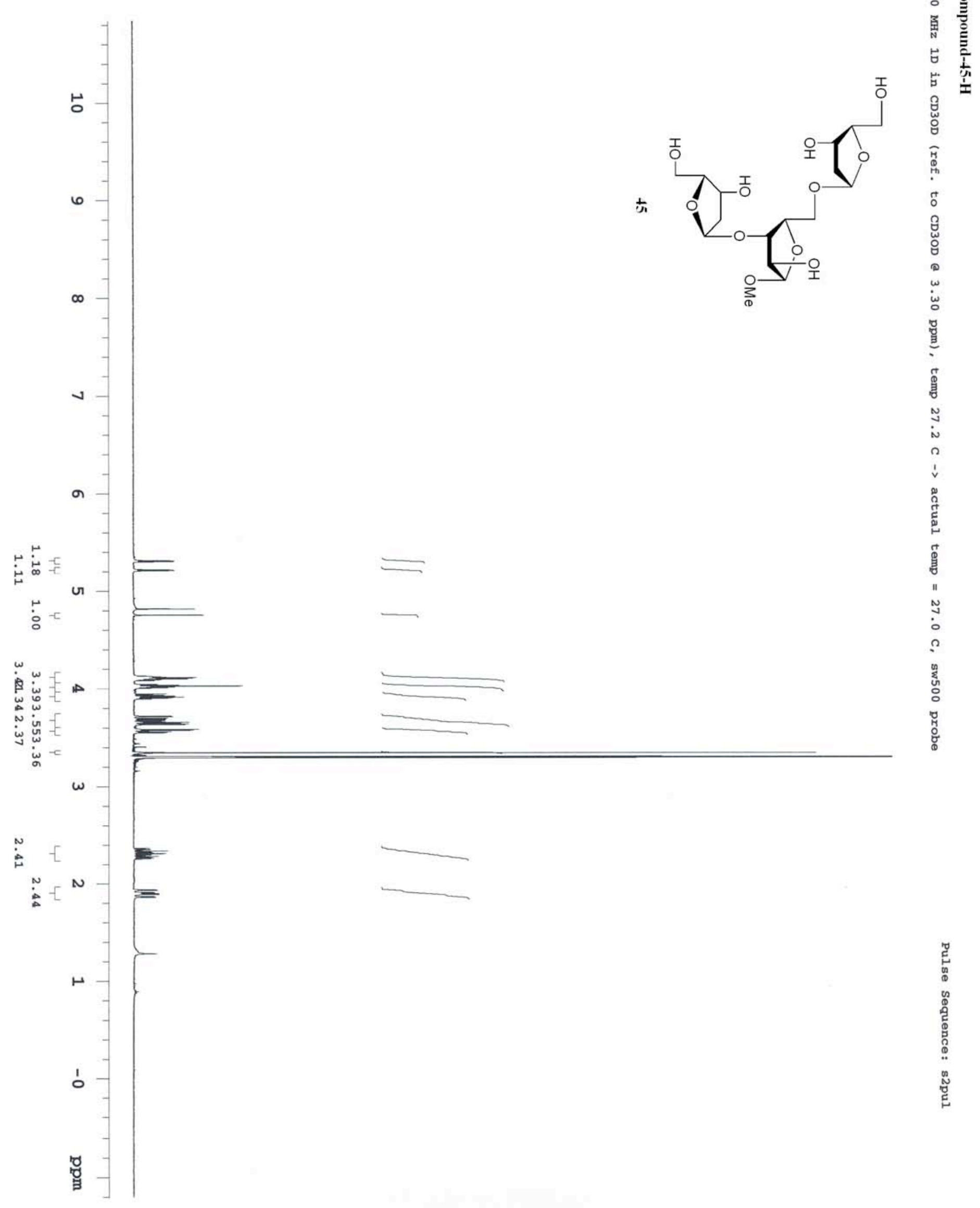



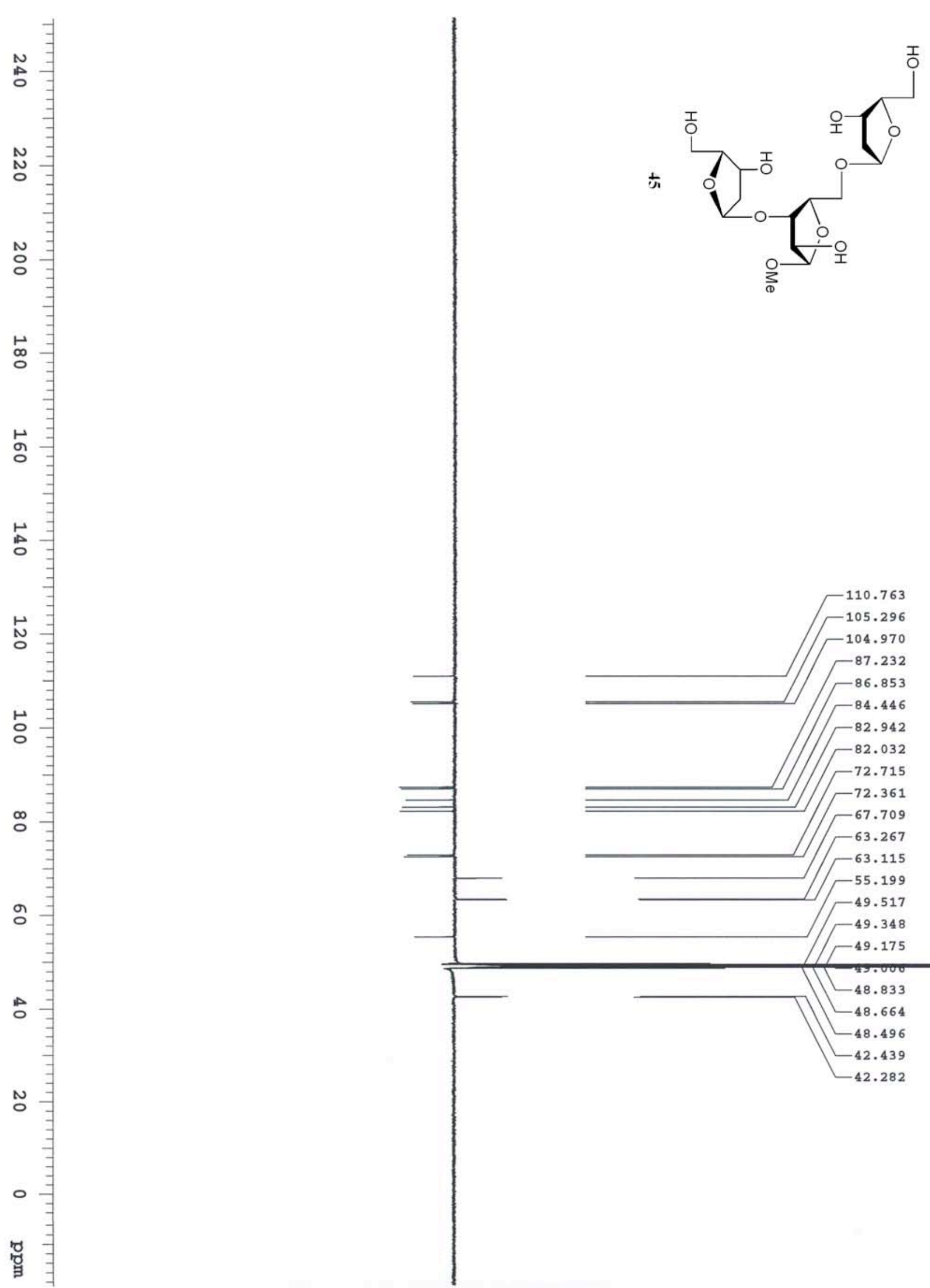

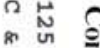

总魚

恕兽

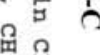

\% ․․

獣

ㅇํำ

+

衰

(⿻)

농

욤

贺:

筧

总兽

$-110.763$

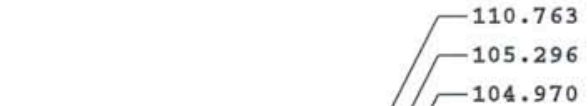

87.930

$-86.85$

$-82.032$

$-72.715$

$-63.267$

$-55.199$

$-49.517$

$-49.34$

$-49.17$

$-48.833$

$-48.664$

$-48.496$

$-42.439$

$-42.282$ 


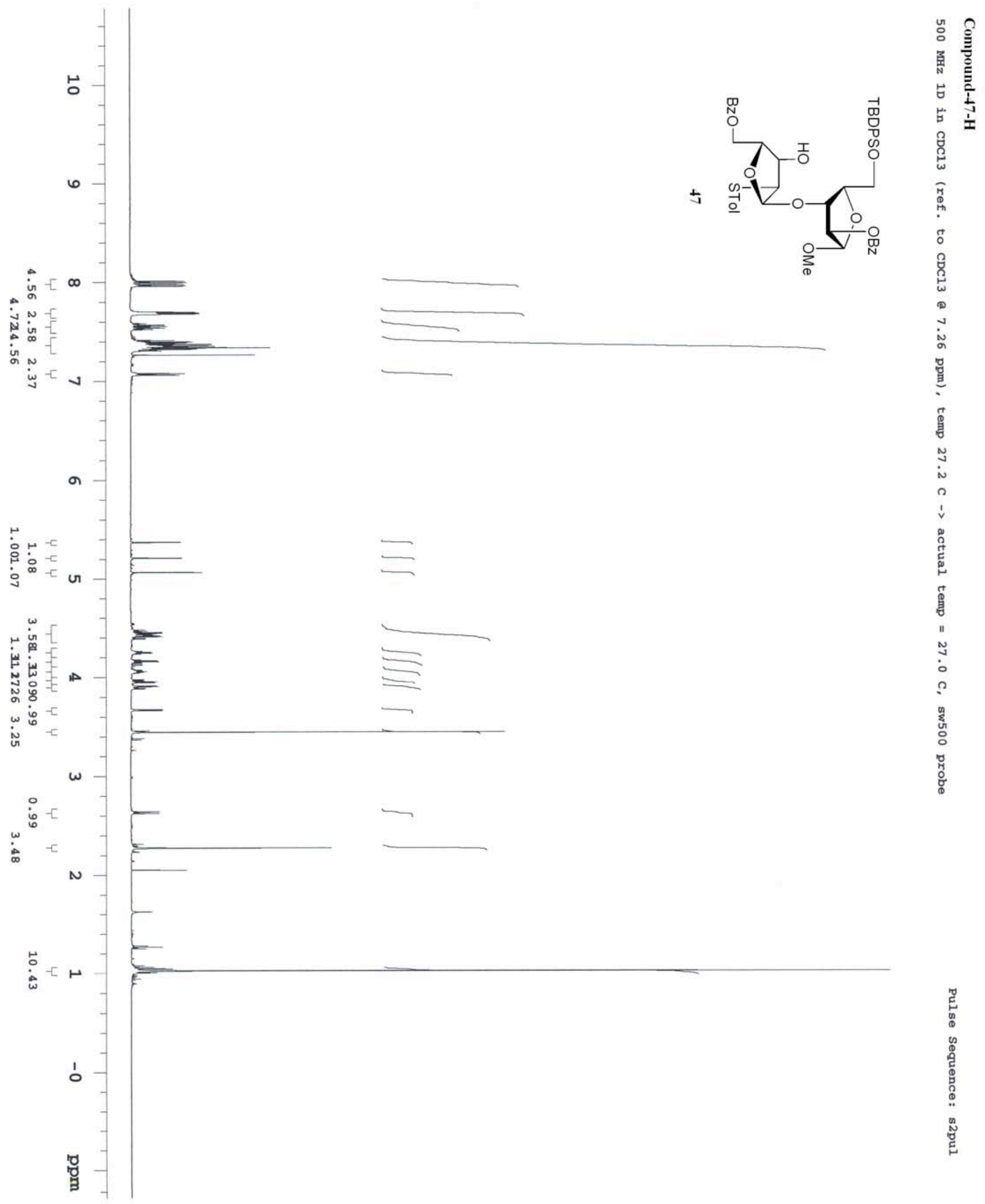




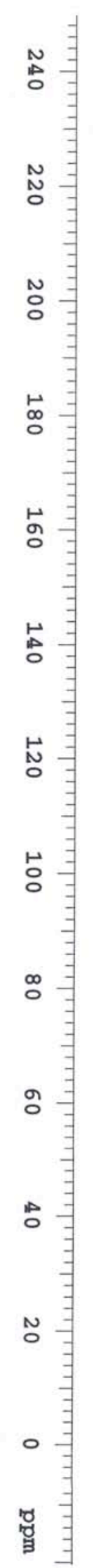

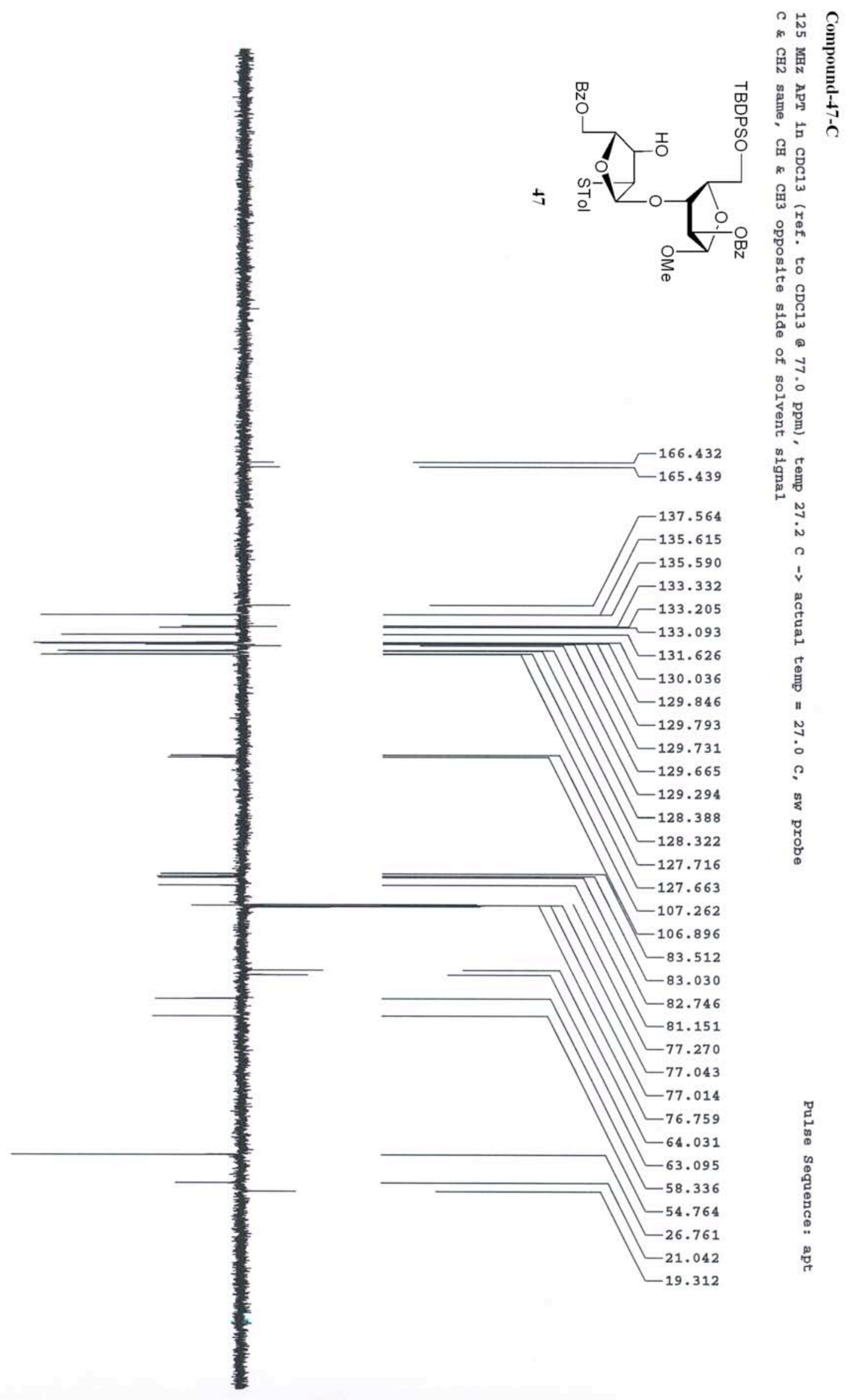




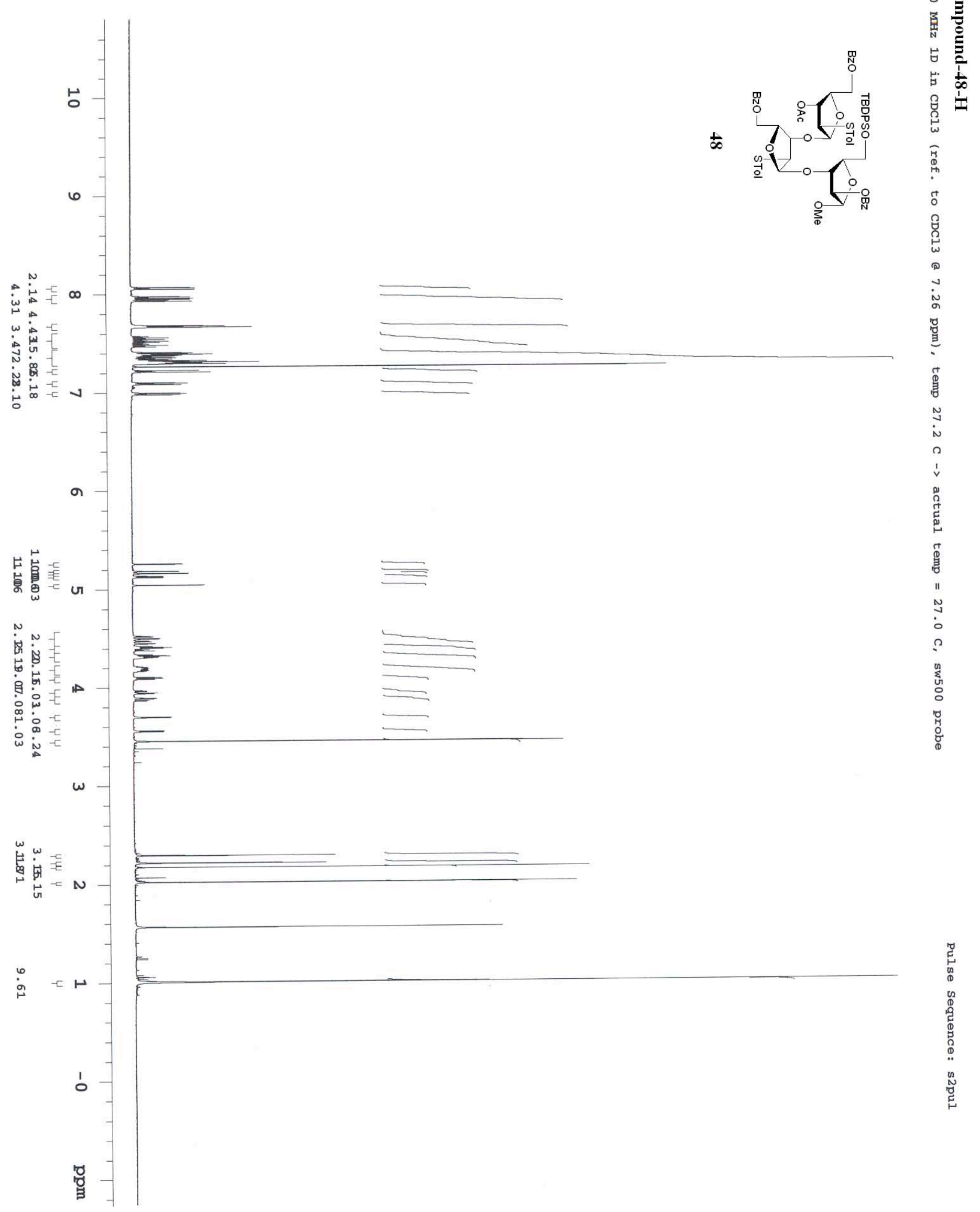




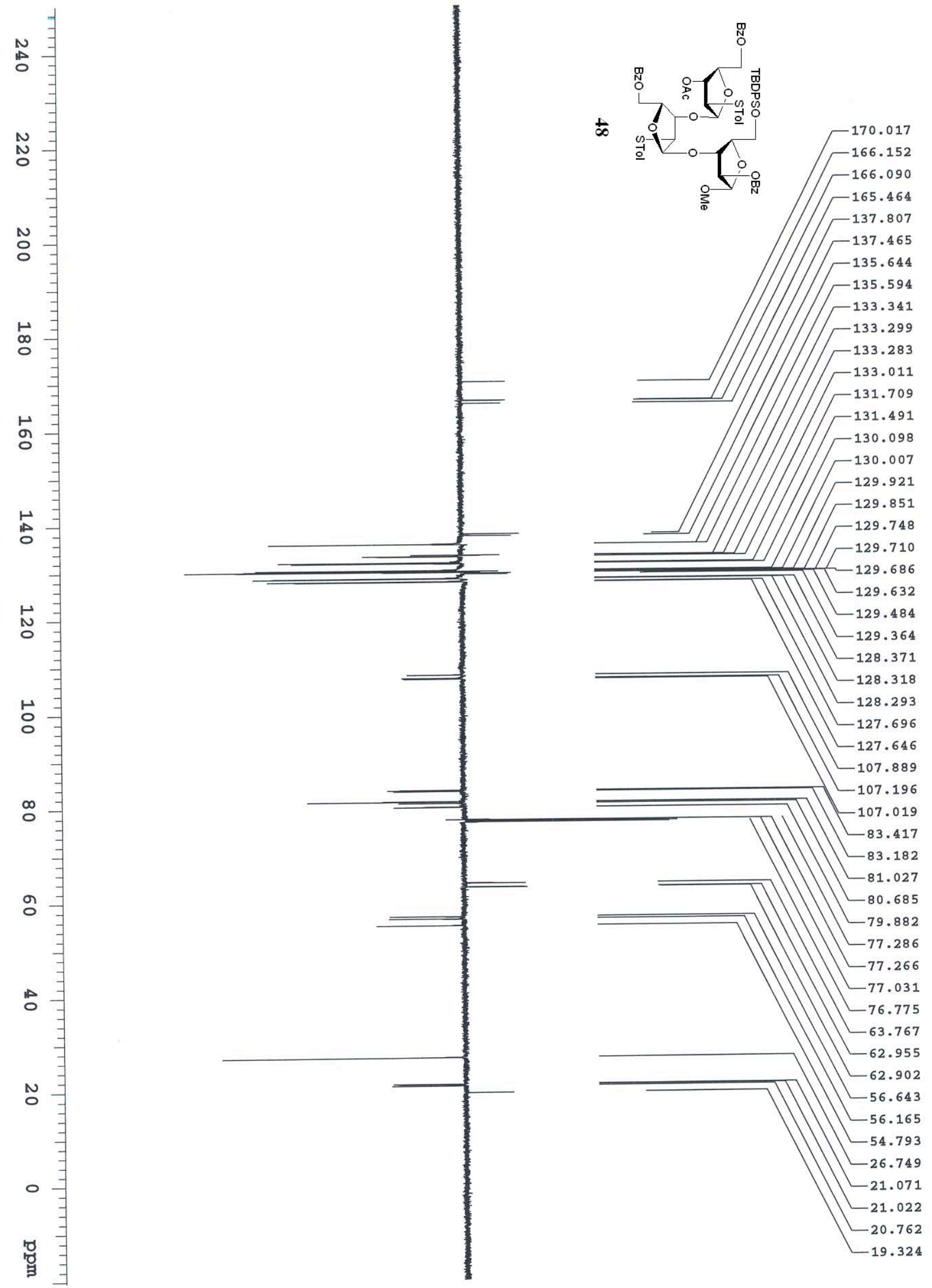




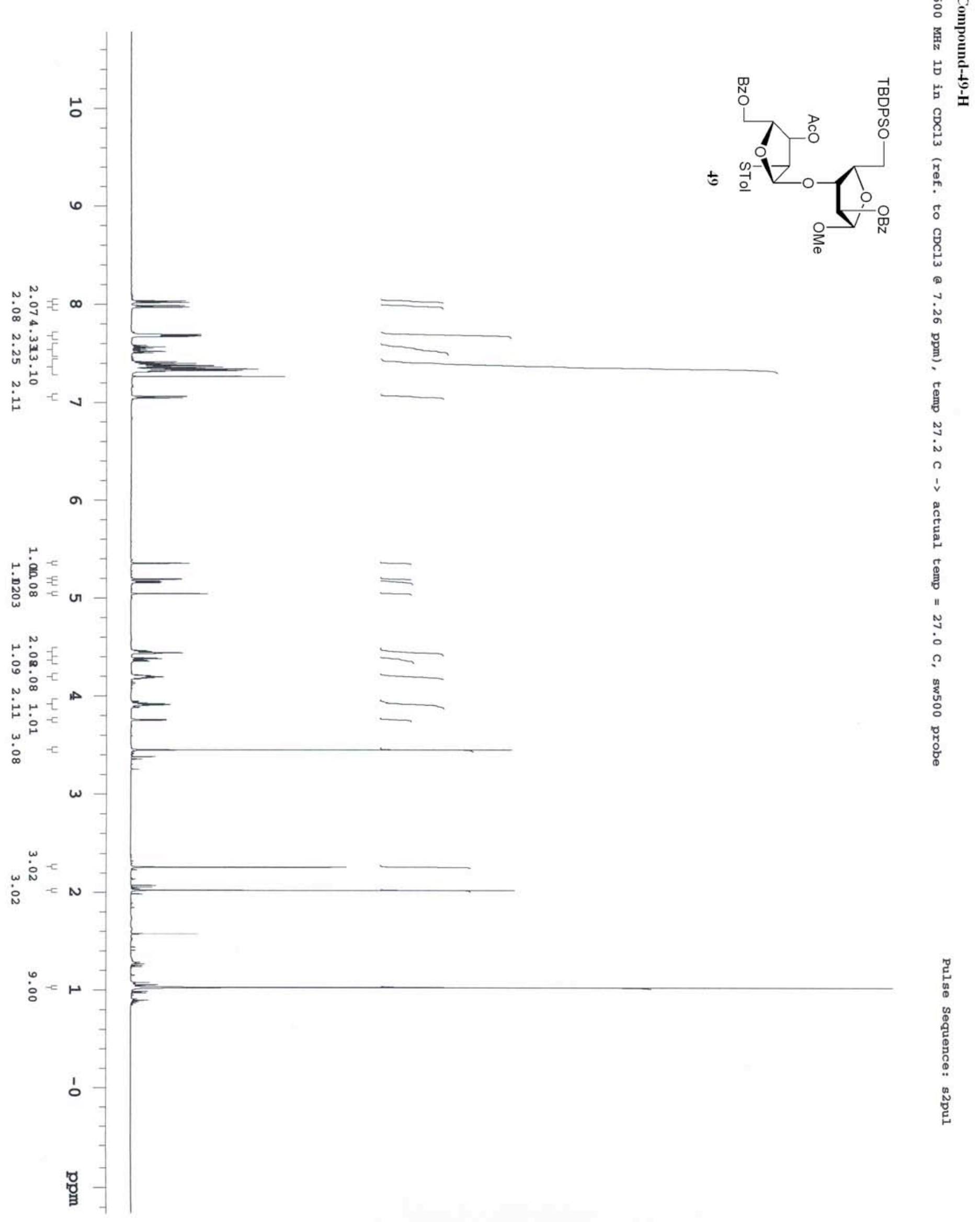



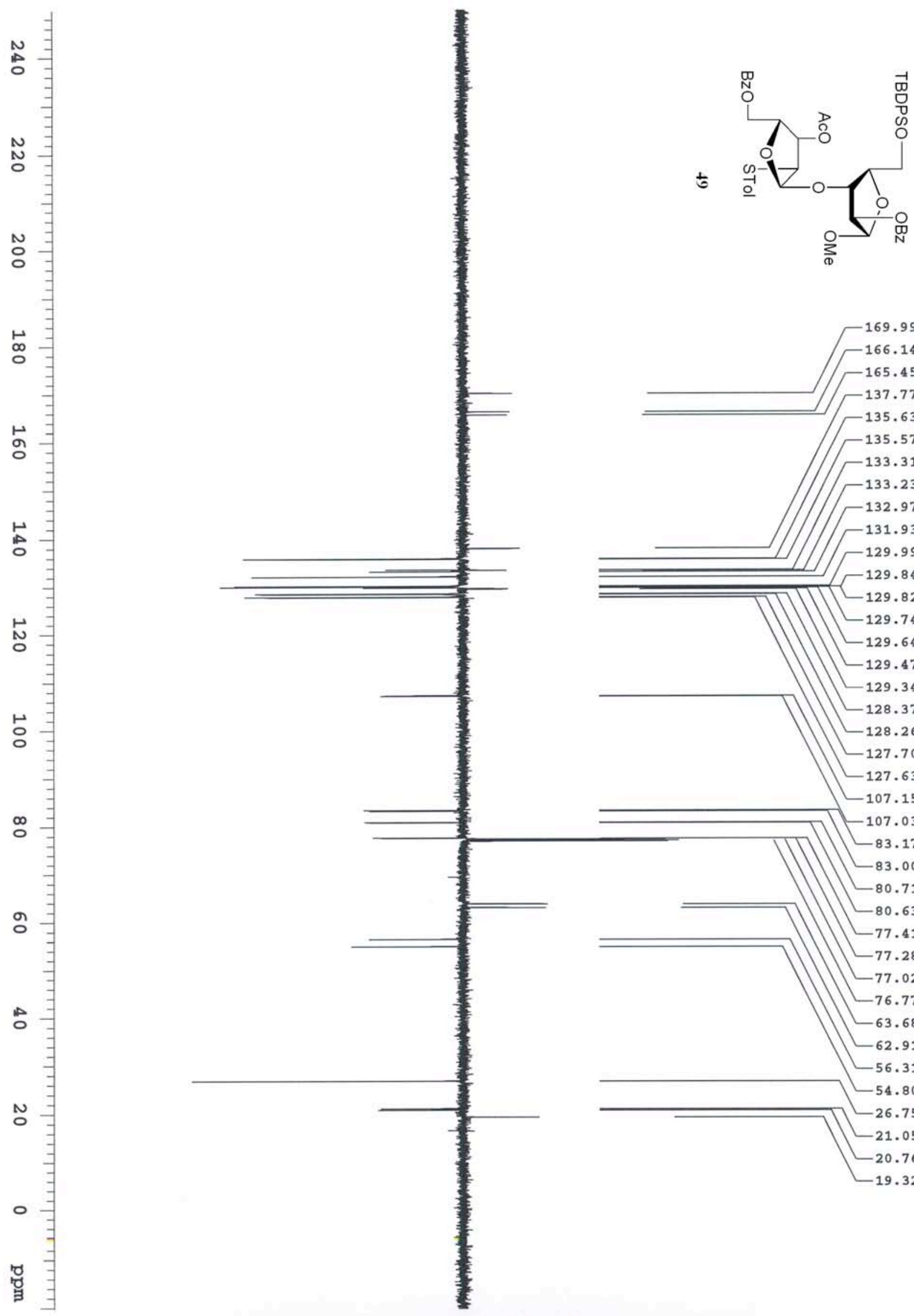

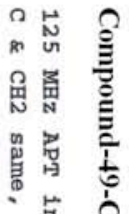

品

ำ

옹

क

莒葛

๑

क ज

음

兽

点兽

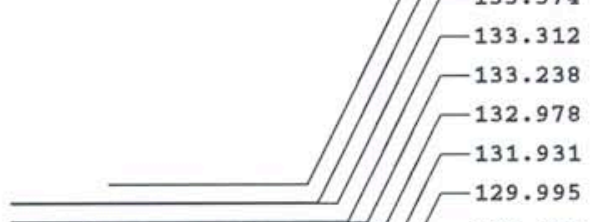

i

in

苦

$-129.842$

129.842

$-129.743$

$-129.649$

$-129.472$

$-129.340$

$-128.375$

$-128.264$

$-127.708$

$-127.638$

$-107.155$

$-107.032$

$-83.178$

$-80.710$

$-80.636$
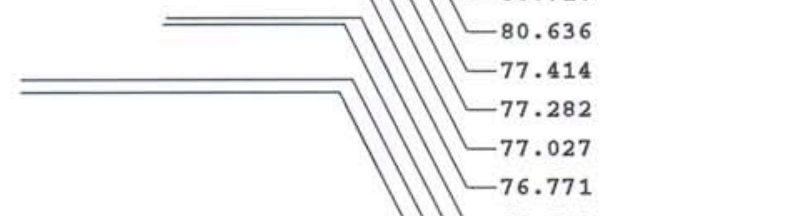

$\sqrt{\square} \longleftarrow 63.680$

$-62.910$

$-56.317$

$-54.805$

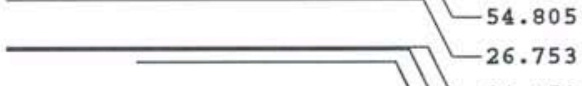

$-21.050$

$-20.762$

$-19.328$ 


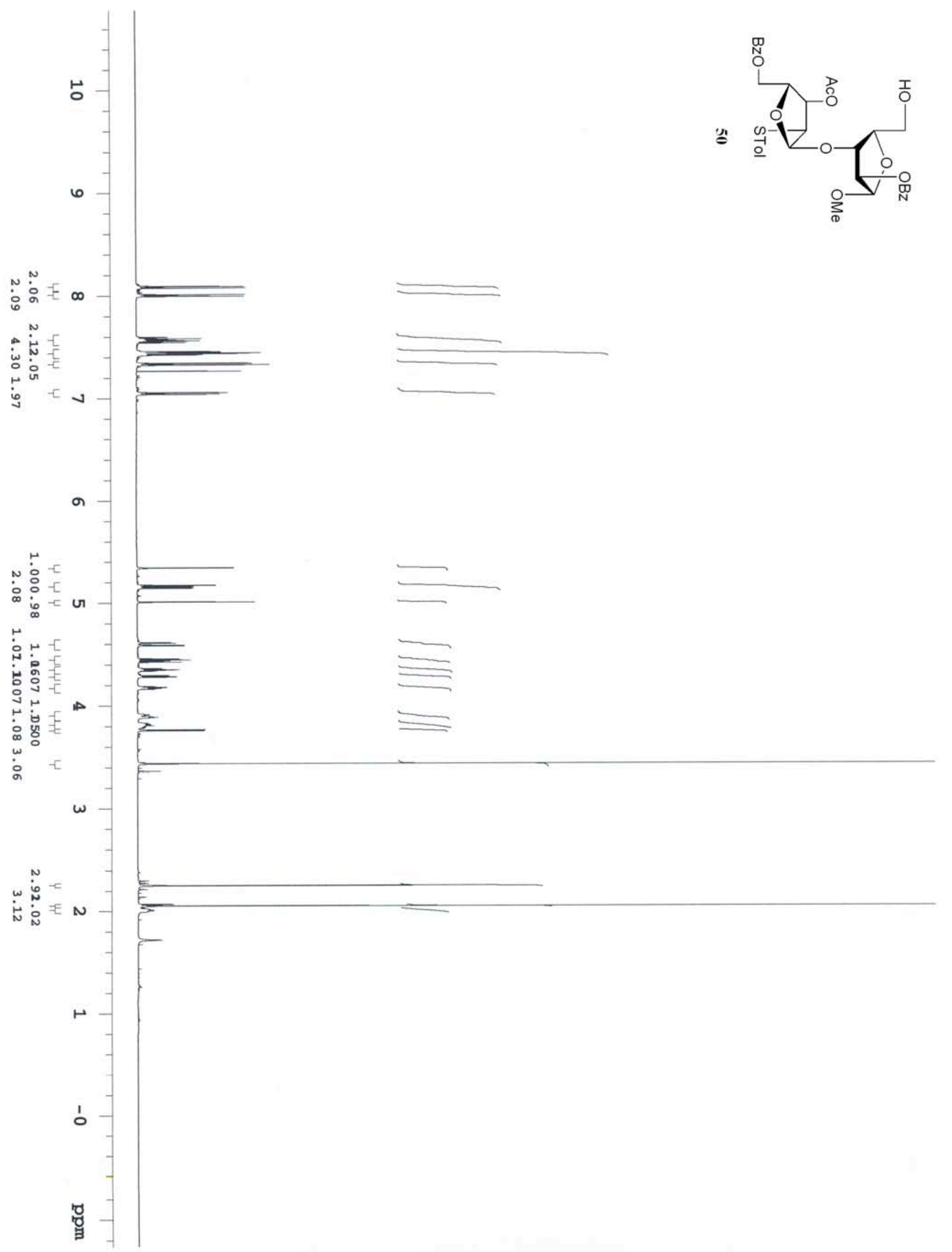



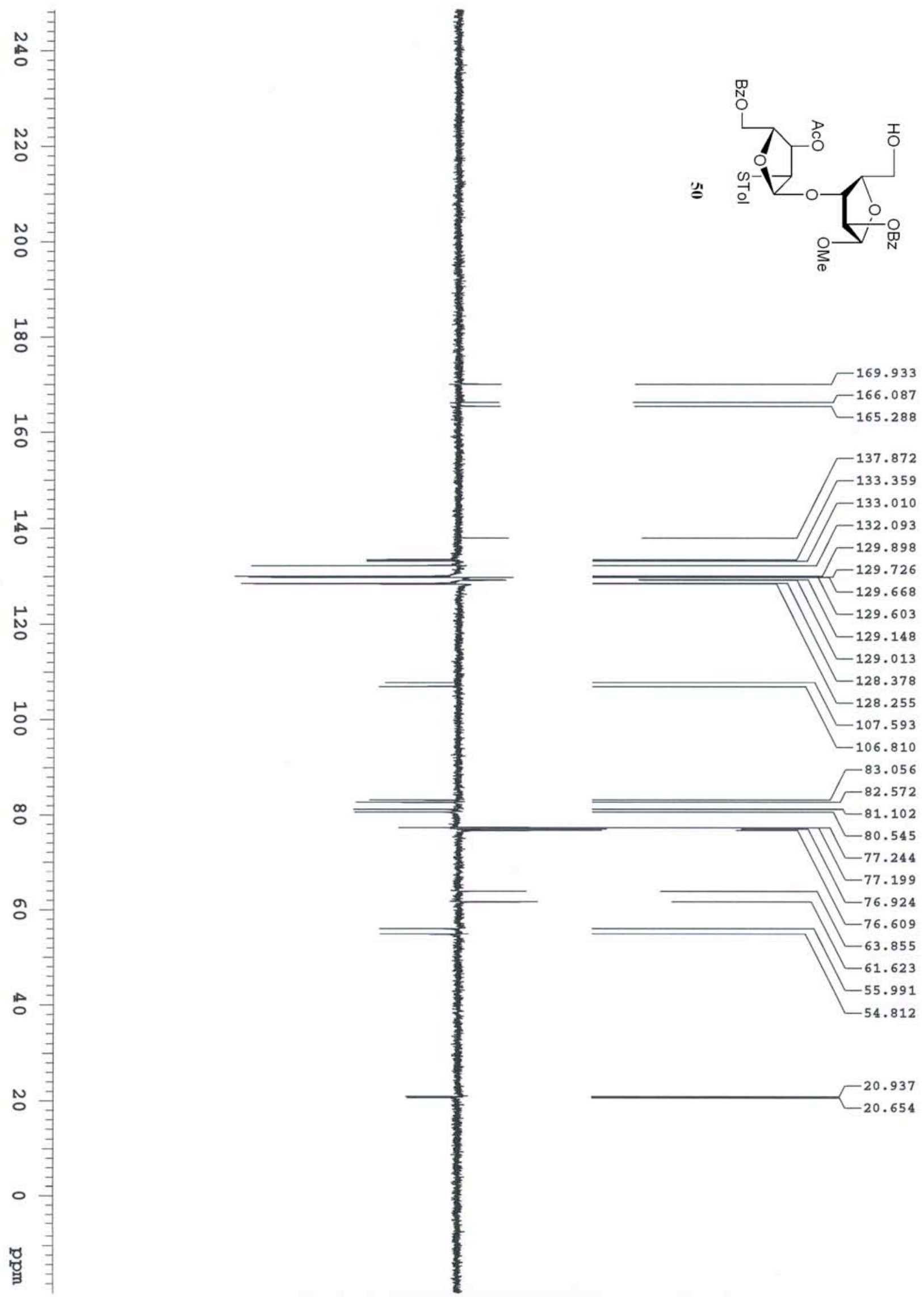

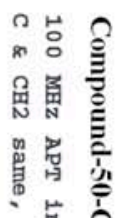

它

요

㽞 w

옹

.

皆

๑) ร

怘莕

ㅇำ

क

4

㝵

总兽

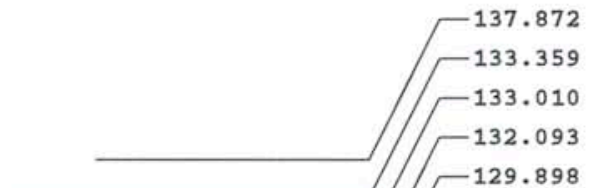

$-129.668$

$-129.603$

$-129.148$

$-129.013$

128.378

$-128.255$

$-107.593$

$-106.810$

$-83.056$

$-80.545$

$-77.244$

$-77.199$

$-76.924$

$-76.609$

$-63.855$

$-61.623$

$-55.991$

$-54.812$

$-20.937$

$-20.654$ 


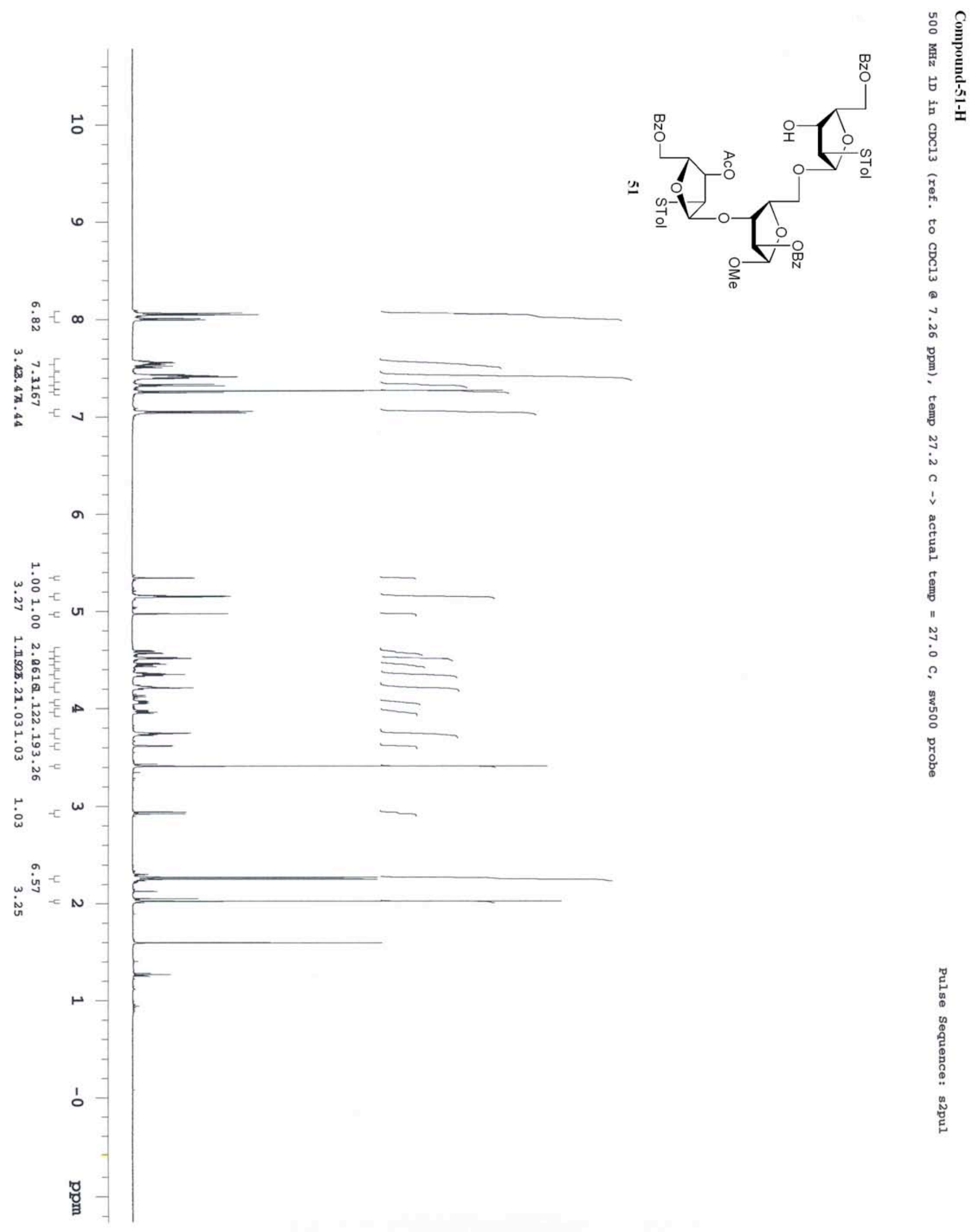



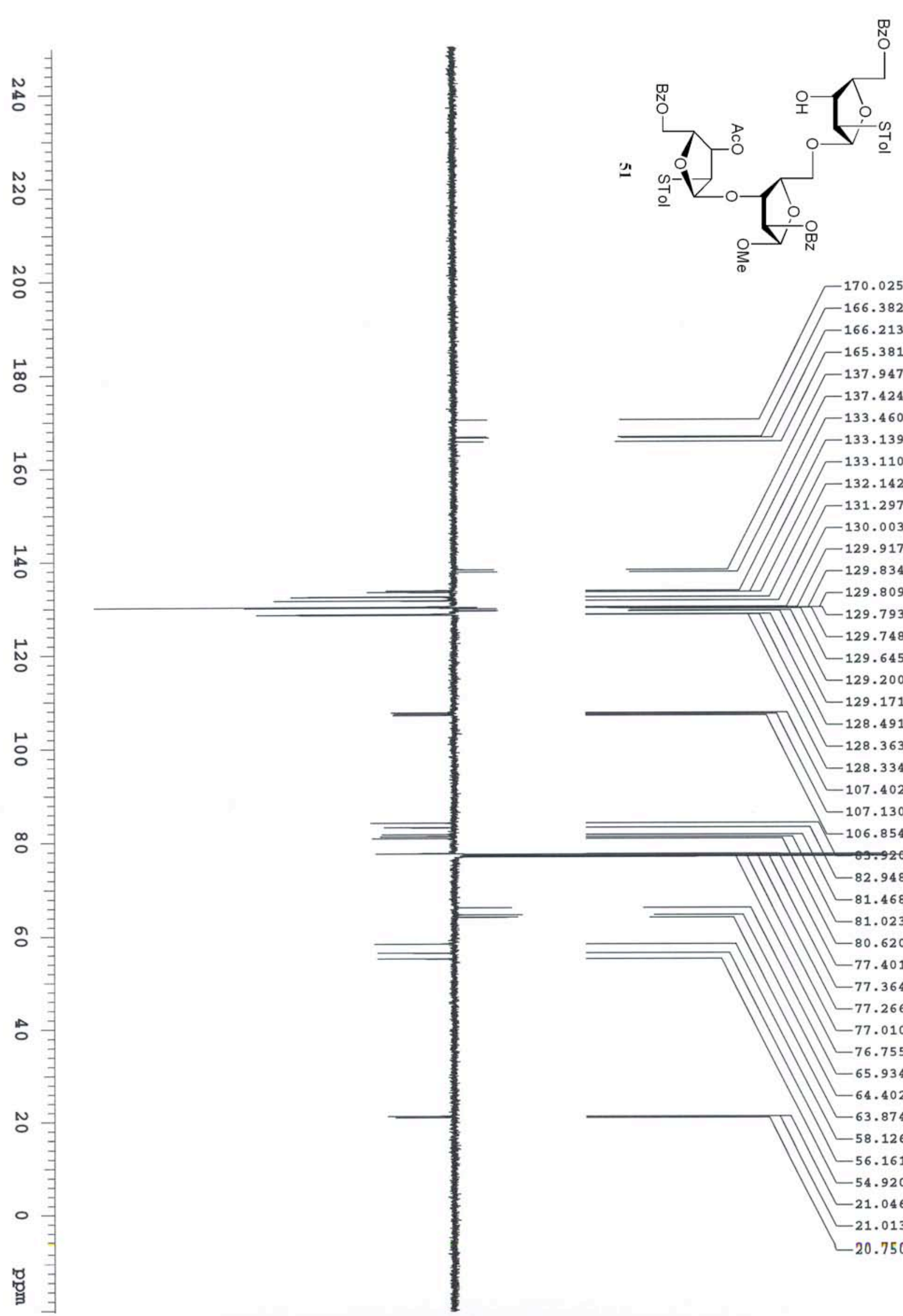

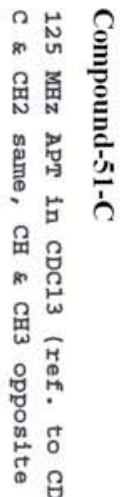

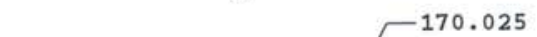

呫

จ จ

का ป

点。

兽

点

$-133.110$

$-132.142$

$-131.297$

-130.003
-129.917

$-129.834$

129.809
-129.793

$-129.748$

$-129.645$

$-129.200$

$-129.171$

$-128.491$

$-128.363$

$-128.334$

$-107.402$

$-107.130$

$-106.854$

$-82.948$

$-81.468$

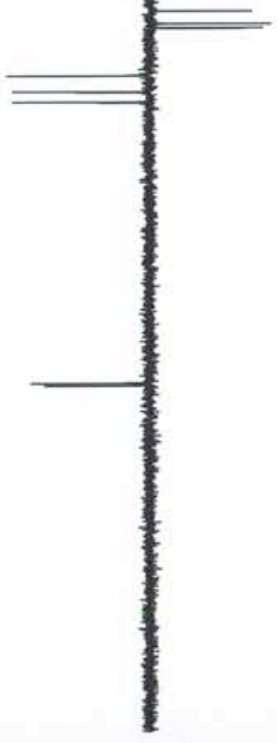

